Supporting Information

\title{
Facile Net Cycloaddition Approach to Optically Active 1,5-Benzothiazepines
}

\author{
Yukihiro Fukata, Keisuke Asano,* and Seijiro Matsubara* \\ Department of Material Chemistry, Graduate School of Engineering, Kyoto \\ University, Kyotodaigaku-Katsura, Nishikyo, Kyoto 615-8510, Japan
}

\section{Contents}

Instrumentation and Chemicals

Experimental Procedure

Table S1

Table S2

Scheme S1

Figure S1 S18

Figure S2 S19

Scheme S2 S20

Scheme S3 S21

Scheme S4 S22

Scheme S5 S23

Characterization Data of Products S24

NMR Spectra $\left({ }^{1} \mathrm{H},{ }^{13} \mathrm{C}\right)$ of Products S37

HPLC Chromatogram Profiles S107

ORTEP Drawings of 3a 


\section{Instrumentation and Chemicals}

${ }^{1} \mathrm{H}$ and ${ }^{13} \mathrm{C}$ Nuclear magnetic resonance spectra were taken on a Varian UNITY INOVA $500\left({ }^{1} \mathrm{H}, 500 \mathrm{MHz} ;{ }^{13} \mathrm{C}, 125.7 \mathrm{MHz}\right)$ spectrometer using tetramethylsilane as an internal standard for ${ }^{1} \mathrm{H}$ NMR $(\delta=0 \mathrm{ppm})$ and $\mathrm{CDCl}_{3}$ as an internal standard for ${ }^{13} \mathrm{C}$ NMR $(\delta=77.0 \mathrm{ppm}) . \quad{ }^{1} \mathrm{H}$ NMR data are reported as follows: chemical shift, multiplicity $(\mathrm{s}=$ singlet, $\mathrm{d}=$ doublet, $\mathrm{t}=$ triplet, $\mathrm{q}=$ quartet, quint $=$ quintet, sext $=$ sextet, sept $=$ septet, br $=$ broad, $\mathrm{m}=$ multiplet $)$, coupling constants $(\mathrm{Hz})$, integration. ${ }^{19} \mathrm{~F}$ NMR spectra were measured on a Varian Mercury $200\left({ }^{19} \mathrm{~F}, 188 \mathrm{MHz}\right)$ spectrometer with hexafluorobenzene as an internal standard $(\delta=0 \mathrm{ppm})$. Mass spectra were recorded on a SHIMADZU GCMS-QP2010 Plus (EI) and a Thermo Scientific Exactive (ESI, APCI) spectrometers. High performance liquid chromatography (HPLC) was performed with a SHIMADZU Prominence. Infrared (IR) spectra were determined on a SHIMADZU IR Affinity-1 spectrometer. Melting points were determined using a YANAKO MP-500D. Optical rotations were measured on a HORIBA SEPA-200. $\mathrm{X}$-ray data were taken on a Rigaku XtalAB mini diffractometer equipped with a CCD detector. TLC analyses were performed by means of Merck Kieselgel $60 \mathrm{~F}_{254}(0.25$ $\mathrm{mm})$ Plates. Visualization was accomplished with UV light (254 $\mathrm{nm})$ and/or such as an aqueous alkaline $\mathrm{KMnO}_{4}$ solution followed by heating.

Flash column chromatography was carried out using Kanto Chemical silica gel (spherical, 40-50 $\mu \mathrm{m}$ ). Unless otherwise noted, commercially available reagents were used without purification. 


\section{Experimental Procedure}

General procedure for asymmetric cycloaddition of $\alpha, \beta$-unsaturated carbonic anhydrides with aminothiophenols

To a 5 -mL vial were added sequentially $\alpha, \beta$-unsaturated substrate $1(0.15 \mathrm{mmol})$, $\mathrm{CHCl}_{3}(0.3 \mathrm{~mL}), 4 \AA \mathrm{MS}(75 \mathrm{mg})$, isothiourea catalyst $6 \mathbf{a}(0.0075 \mathrm{mmol})$, and aminothiophenol $2(0.15 \mathrm{mmol})$. The mixture was stirred in an oil bath maintained at $25{ }^{\circ} \mathrm{C}$ for $24 \mathrm{~h}$. The reaction mixture was subsequently diluted with hexane/EtOAc $(\mathrm{v} / \mathrm{v}=5: 1)$, passed through a short silica gel pad to remove $6 \mathbf{a}$, and concentrated in vacuo to give the crude 1,5-benzothiazepine $\mathbf{3}$ as a pale yellow solid. Purification of the crude product by flash silica gel column chromatography using hexane/EtOAc (v/v $=3: 1$ ) as an eluent afforded the corresponding 1,5-benzothiazepines (3) as a white solid.

Racemic compounds were prepared using racemic isothiourea catalyst $6 \mathbf{a}$.

\section{Procedure for preparation of benzotetramisole catalyst $6 a^{1}$}

Benzotetramisole catalyst $\mathbf{6 a}$ was prepared by the literature procedure. ${ }^{1}$ To the solution of $(R)-(+)$-phenylglycinol $(1.4 \mathrm{~g}, 10 \mathrm{mmol})$ in $i$ - $\operatorname{Pr}_{2} \operatorname{EtN}(2.7 \mathrm{~mL}, 15 \mathrm{mmol})$ was added 2-chlorobenzothiazole $(1.7 \mathrm{~g}, 10 \mathrm{mmol})$, and the mixture was heated at $135{ }^{\circ} \mathrm{C}$ for $24 \mathrm{~h}$. After cooling the mixture to $40{ }^{\circ} \mathrm{C}$, the viscous reaction mixture was treated with $\mathrm{CH}_{2} \mathrm{Cl}_{2}(5 \mathrm{~mL})$, and left at ambient temperature overnight to dissolve the solid completely. The diluted reaction mixture was applied directly to a chromatographic column and eluted with $\mathrm{CH}_{2} \mathrm{Cl}_{2} / i-\mathrm{PrOH}$ (v/v = 20/1) to afford (R)- $N$-(triazolyl-2)-2-hydroxy-1-phenylethylamine (white solid, $2.4 \mathrm{~g}, 90 \%$ yield).

A solution of $(R)-N$-(triazolyl-2)-2-hydroxy-1-phenylethylamine $(2.4 \mathrm{~g}, 9.0 \mathrm{mmol})$ in anhydrous $\mathrm{CH}_{2} \mathrm{Cl}_{2}(80 \mathrm{~mL})$ was cooled to $0{ }^{\circ} \mathrm{C}$ under argon atmosphere and treated with $\mathrm{NEt}_{3}(3.7 \mathrm{~mL}, 27 \mathrm{mmol})$ followed by $\mathrm{MsCl}(1.1 \mathrm{~mL}, 13.5 \mathrm{mmol})$. The mixture was stirred at $0{ }^{\circ} \mathrm{C}$ for $1 \mathrm{~h}$ and then warmed up to ambient temperature. Methanol $(0.5$ $\mathrm{mL})$ was added to decompose the excess $\mathrm{MsCl}$. $\mathrm{NEt}_{3}(10 \mathrm{~mL})$ was added, and the mixture was refluxed overnight. The cooled mixture was washed with water $(10 \mathrm{~mL})$, dried over $\mathrm{Na}_{2} \mathrm{SO}_{4}$, and evaporated in vacuo. The crude product was purified by column chromatography with hexane $/ i-\mathrm{PrOH} / \mathrm{NEt}_{3}(\mathrm{v} / \mathrm{v} / \mathrm{v}=100 / 5 / 1)$ as an eluent to give a white solid. The compound was recrystallized from EtOAc/hexane to produce $\mathbf{6 a}$ (white solid, $1.36 \mathrm{~g}, 60 \%$ yield). 


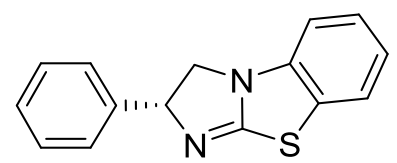

White solid. $[\alpha]_{\mathrm{D}}^{18}+252.5(c 1.00, \mathrm{MeOH})$.

${ }^{1} \mathrm{H}$ NMR $\left(\mathrm{CDCl}_{3}\right) \delta 7.38-7.37(\mathrm{~m}, 4 \mathrm{H}), 7.34(\mathrm{dd}, J=8.0,1.0 \mathrm{~Hz}, 1 \mathrm{H}), 7.30(\mathrm{~m}, 1 \mathrm{H})$, $7.22(\mathrm{dt}, J=8.0,1.0 \mathrm{~Hz}, 1 \mathrm{H}), 7.01(\mathrm{dt}, J=8.0,1.0 \mathrm{~Hz}, 1 \mathrm{H}), 6.72(\mathrm{dd}, J=8.0,1.0 \mathrm{~Hz}$, $1 \mathrm{H}), 5.70$ (dd, $J=10.5,8.0 \mathrm{~Hz}, 1 \mathrm{H}), 4.35$ (dd, $J=10.5,9.0 \mathrm{~Hz}, 1 \mathrm{H}), 3.78$ (dd, $J=9.0$, $8.0 \mathrm{~Hz}, 1 \mathrm{H}) .{ }^{13} \mathrm{C} \mathrm{NMR}\left(\mathrm{CDCl}_{3}\right) \delta 167.2,142.4,136.7,128.8,127.8,127.4,126.7$, 126.4, 123.3, 121.8, 108.8, 74.4, 52.6. Mp. 94.5-95.5 ${ }^{\circ} \mathrm{C} . \quad$ TLC: $\mathrm{R}_{\mathrm{f}} 0.30$ (hexane/i-PrOH = 20:1). IR (KBr): 3024, 2871, 1596, 1574, 1452, 1376, 1217, 746 $\mathrm{cm}^{-1}$. HRMS Calcd for $\mathrm{C}_{15} \mathrm{H}_{13} \mathrm{~N}_{2} \mathrm{~S}:[\mathrm{M}+\mathrm{H}]^{+}, 253.0794$. Found: $m / z$ 253.0788.

\section{General procedure for preparation of $\alpha, \beta$-unsaturated carbonic anhydrides $1 a-1 i$}

To a stirred solution of an $\alpha, \beta$-unsaturated carboxylic acid (1.0 equiv) in $\mathrm{CH}_{2} \mathrm{Cl}_{2}$ $(0.72 \mathrm{M})$ was slowly added $i-\operatorname{Pr}_{2} \mathrm{EtN}$ (1.5 equiv) and isopropyl chloroformate (1.1 equiv) at $0{ }^{\circ} \mathrm{C}$, and the mixture was stirred at $0{ }^{\circ} \mathrm{C}$ for $30 \mathrm{~min} . \quad \mathrm{H}_{2} \mathrm{O}$ was added to the solution, and the mixture was extracted with $\mathrm{CH}_{2} \mathrm{Cl}_{2}$. The combined organic layers were washed with brine, dried over $\mathrm{Na}_{2} \mathrm{SO}_{4}$, and concentrated in vacuo. Purification by flash silica gel column chromatography using hexane/EtOAc $(\mathrm{v} / \mathrm{v}=10 / 1)$ as an eluent gave the corresponding carbonic anhydride.

(E)-Cinnamic (isopropyl carbonic) anhydride ((E)-1a).<smiles>CC(C)OC(=O)OC(=O)/C=C/c1ccccc1</smiles>

Colorless oil; $70 \%$ yield $(0.95 \mathrm{~g})$.

${ }^{1} \mathrm{H}$ NMR $\left(\mathrm{CDCl}_{3}\right) \delta 7.82(\mathrm{~d}, J=16.0 \mathrm{~Hz}, 1 \mathrm{H}), 7.55(\mathrm{~m}, 2 \mathrm{H}), 7.42(\mathrm{~m}, 3 \mathrm{H}), 6.42(\mathrm{~d}, J=$ $16.0 \mathrm{~Hz}, 1 \mathrm{H}), 5.05(\mathrm{sept}, J=6.0 \mathrm{~Hz}, 1 \mathrm{H}), 1.39(\mathrm{~d}, J=6.0 \mathrm{~Hz}, 6 \mathrm{H}) .{ }^{13} \mathrm{C} \mathrm{NMR}\left(\mathrm{CDCl}_{3}\right)$ $\delta 161.1,148.9,148.6,133.5,131.3,129.0,128.5,115.4,74.2,21.5$. TLC: $\mathrm{R}_{\mathrm{f}} 0.36$ (hexane/EtOAc $=10: 1) . \quad$ IR (neat): 2985, 1792, 1730, 1632, 1450, 1240, 1154, 1071, 903, 763, $697 \mathrm{~cm}^{-1}$. HRMS Calcd for $\mathrm{C}_{13} \mathrm{H}_{14} \mathrm{O}_{4} \mathrm{Na}$ : $[\mathrm{M}+\mathrm{Na}]^{+}, 257.0784$. Found: $\mathrm{m} / z$ 257.0778 . 
(E)-(Isopropyl carbonic) 3-(p-tolyl)acrylic anhydride (1b).<smiles>Cc1ccc(/C=C/C(=O)OC(=O)OC(C)C)cc1</smiles>

Colorless oil; $75 \%$ yield $(0.93 \mathrm{~g})$.

${ }^{1} \mathrm{H} \mathrm{NMR}\left(\mathrm{CDCl}_{3}\right) \delta 7.80(\mathrm{~d}, J=16.0 \mathrm{~Hz}, 1 \mathrm{H}), 7.44(\mathrm{~d}, J=8.0 \mathrm{~Hz}, 2 \mathrm{H}), 7.22(\mathrm{~d}, J=8.0$ $\mathrm{Hz}, 2 \mathrm{H}), 6.36(\mathrm{~d}, J=16.0 \mathrm{~Hz}, 1 \mathrm{H}), 5.05$ (sept, $J=6.0 \mathrm{~Hz}, 1 \mathrm{H}), 2.39$ (s, 3H), 1.39 (d, $J=$ $6.0 \mathrm{~Hz}, 6 \mathrm{H}) .{ }^{13} \mathrm{C} \mathrm{NMR}\left(\mathrm{CDCl}_{3}\right) \delta 161.3,149.0,148.8,142.0,130.9,129.8,128.6$, 114.3, 74.2, 21.6, 21.5. TLC: $\mathrm{R}_{\mathrm{f}} 0.44$ (hexane/EtOAc = 10:1). IR (neat): 2985, 1793, 1735, 1631, 1242, 1157, 1094, 1072, 906, $812 \mathrm{~cm}^{-1}$. HRMS Calcd for $\mathrm{C}_{14} \mathrm{H}_{16} \mathrm{O}_{4} \mathrm{Na}$ : $[\mathrm{M}+\mathrm{Na}]^{+}, 271.0941$. Found: $m / z, 271.0943$.

(E)-(Isopropyl carbonic) 3-(o-tolyl)acrylic anhydride (1c).<smiles>Cc1ccccc1/C=C/C(=O)OC(=O)OC(C)C</smiles>

Colorless oil; $77 \%$ yield (0.96 g).

${ }^{1} \mathrm{H}$ NMR $\left(\mathrm{CDCl}_{3}\right) \delta 8.12(\mathrm{~d}, J=16.0 \mathrm{~Hz}, 1 \mathrm{H}), 7.56(\mathrm{~d}, J=7.5 \mathrm{~Hz}, 1 \mathrm{H}), 7.32(\mathrm{t}, J=7.5$ Hz, 1H), 7.26-7.22 (m, 2H), 6.34 (d, $J=16.0 \mathrm{~Hz}, 1 \mathrm{H}), 5.06$ (sept, $J=6.5 \mathrm{~Hz}, 1 \mathrm{H}), 2.46$ $(\mathrm{s}, 3 \mathrm{H}), 1.40(\mathrm{~d}, J=6.5 \mathrm{~Hz}, 1 \mathrm{H}) .{ }^{13} \mathrm{C} \mathrm{NMR}\left(\mathrm{CDCl}_{3}\right) \delta 162.3,148.7,146.5,138.3$, $132.5,131.02,131.99,126.7,126.5,116.3,71.3,21.5,19.8$. TLC: $R_{f} 0.44$ (hexane/EtOAc $=10: 1) . \quad$ IR (neat): 2985, 1797, 1735, 1628, 1466, 1240, 1160, 1075, 906, $761 \mathrm{~cm}^{-1}$. HRMS Calcd for $\mathrm{C}_{14} \mathrm{H}_{16} \mathrm{O}_{4} \mathrm{Na}$ : $[\mathrm{M}+\mathrm{Na}]^{+}, 271.0941$. Found: $\mathrm{m} / \mathrm{z}$ 271.0942 .

(E)-(Isopropyl carbonic) 3-(4-methoxyphenyl)acrylic anhydride (1d).<smiles>COc1ccc(/C=C/C(=O)OC(=O)OC(C)C)cc1</smiles>

Colorless oil; $38 \%$ yield $(0.50 \mathrm{~g})$.

${ }^{1} \mathrm{H}$ NMR $\left(\mathrm{CDCl}_{3}\right) \delta 7.78(\mathrm{~d}, J=16.0 \mathrm{~Hz}, 1 \mathrm{H}), 7.51(\mathrm{~m}, 2 \mathrm{H}), 6.92(\mathrm{~m}, 2 \mathrm{H}), 6.28(\mathrm{~d}, J=$ $16.0 \mathrm{~Hz}, 1 \mathrm{H}), 5.04$ (sept, $J=6.5 \mathrm{~Hz}, 1 \mathrm{H}), 3.85(\mathrm{~s}, 3 \mathrm{H}), 1.38(\mathrm{~d}, J=6.5 \mathrm{~Hz}, 6 \mathrm{H}) .{ }^{13} \mathrm{C}$ NMR $\left(\mathrm{CDCl}_{3}\right) \delta 162.2,161.4,148.9,148.7,130.4,126.3,114.5,112.7,74.1,55.4,21.5$. TLC: $R_{\mathrm{f}} 0.20$ (hexane/EtOAc = 10:1). IR (neat): 2985, 1797, 1732, 1628, 1602, 1514, 1258, 1154, 1094, 906, $828 \mathrm{~cm}^{-1}$. HRMS Calcd for $\mathrm{C}_{14} \mathrm{H}_{16} \mathrm{O}_{5} \mathrm{Na}:[\mathrm{M}+\mathrm{Na}]^{+}, 287.0890$. 
Found: $m / z 287.0879$.

(E)-3-(4-(Trifluoromethyl)phenyl)acrylic (isopropyl carbonic) anhydride (1e).<smiles>CC(C)OC(=O)OC(=O)/C=C/c1ccc(C(F)(F)F)cc1</smiles>

White solid; $77 \%$ yield (1.2 g).

${ }^{1} \mathrm{H}$ NMR $\left(\mathrm{CDCl}_{3}\right) \delta 7.82(J=16.0 \mathrm{~Hz}, 1 \mathrm{H}), 7.69-7.64(\mathrm{~m}, 4 \mathrm{H}), 6.49(\mathrm{~d}, J=16.0 \mathrm{~Hz}$, $1 \mathrm{H}), 5.05(\mathrm{sept}, J=6.0 \mathrm{~Hz}, 1 \mathrm{H}), 1.38(\mathrm{~d}, J=6.0 \mathrm{~Hz}, 6 \mathrm{H}) .{ }^{13} \mathrm{C} \mathrm{NMR}\left(\mathrm{CDCl}_{3}\right) \delta 160.6$, 148.4, 146.7, 136.8, $132.2(\mathrm{q}, J=33.1 \mathrm{~Hz}), 128.6,126.0(\mathrm{q}, J=3.4 \mathrm{~Hz}), 123.5$ (q, $J=$ $272.6 \mathrm{~Hz}), \quad 118.1,74.6, \quad 21.4 . \quad{ }^{19} \mathrm{~F} \quad \mathrm{NMR}\left(\mathrm{CDCl}_{3}\right) \quad \delta \quad 98.8 . \quad$ TLC: $\mathrm{R}_{\mathrm{f}} \quad 0.33$ (hexane/EtOAc $=10: 1) . \quad$ IR $(\mathrm{KBr}): 2992,1802,1762,1633,1326,1239,1167,1122$, 1058, 907, 837, 823, $758 \mathrm{~cm}^{-1}$. HRMS Calcd for $\mathrm{C}_{14} \mathrm{H}_{13} \mathrm{~F}_{3} \mathrm{O}_{4} \mathrm{Na}$ : $[\mathrm{M}+\mathrm{Na}]^{+}, 325.0658$. Found: $m / z 325.0644$.

(E)-3-(4-Bromophenyl)acrylic (isopropyl carbonic) anhydride (1f).<smiles>CC(C)OC(=O)OC(=O)/C=C/c1ccc(Br)cc1</smiles>

White solid; $36 \%$ yield $(0.57 \mathrm{~g})$.

${ }^{1} \mathrm{H}$ NMR $\left(\mathrm{CDCl}_{3}\right) \delta 7.75(\mathrm{~d}, J=15.5 \mathrm{~Hz}, 1 \mathrm{H}), 7.55(\mathrm{~m}, 2 \mathrm{H}), 7.41(\mathrm{~m}, 2 \mathrm{H}), 6.40(\mathrm{~d}, J=$ $15.5 \mathrm{~Hz}, 1 \mathrm{H}), 5.05$ (sept, $J=6.0 \mathrm{~Hz}, 1 \mathrm{H}), 1.39(\mathrm{~d}, J=6.0 \mathrm{~Hz}, 6 \mathrm{H}) .{ }^{13} \mathrm{C} \mathrm{NMR}\left(\mathrm{CDCl}_{3}\right)$ $\delta 160.9,148.5,147.4,132.5,132.4,129.8,125.8,116.2,74.4,21.5$. TLC: $\mathrm{R}_{\mathrm{f}} 0.36$ (hexane/EtOAc = 10:1). IR $(\mathrm{KBr}): 2981,1788,1730,1634,1586,1488,1375,1248$, 1076, 1006, 910, $819 \mathrm{~cm}^{-1}$. HRMS Calcd for $\mathrm{C}_{13} \mathrm{H}_{13} \mathrm{BrO}_{4} \mathrm{Na}:[\mathrm{M}+\mathrm{Na}]^{+}, 334.9889$. Found: $m / z 334.9880$.

(E)-3-(Furan-2-yl)acrylic (isopropyl carbonic) anhydride (1g).<smiles>CC(C)OC(=O)OC(=O)/C=C/c1ccco1</smiles>

Colorless oil; $81 \%$ yield $(0.91 \mathrm{~g})$.

${ }^{1} \mathrm{H} \mathrm{NMR}\left(\mathrm{CDCl}_{3}\right) \delta 7.53(\mathrm{~d}, J=16.5 \mathrm{~Hz}, 1 \mathrm{H}), 7.53(\mathrm{~m}, 1 \mathrm{H}) 6.72(\mathrm{~d}, J=3.5 \mathrm{~Hz}, 1 \mathrm{H})$, $6.51(\mathrm{dd}, J=3.5,2.0 \mathrm{~Hz}, 1 \mathrm{H}), 6.27(\mathrm{~d}, J=16.5 \mathrm{~Hz}, 1 \mathrm{H}), 5.03$ (sept, $J=6.5 \mathrm{~Hz}, 1 \mathrm{H})$, 
$1.37(\mathrm{~d}, J=6.5 \mathrm{~Hz}, 6 \mathrm{H}) . \quad{ }^{13} \mathrm{C} \mathrm{NMR}\left(\mathrm{CDCl}_{3}\right) \delta 161.2,150.2,148.7,145.9,134.5,117.1$, 112.8, 112.7, 74.1, 21.5. TLC: $\mathrm{R}_{\mathrm{f}} 0.21$ (hexane/EtOAc = 10:1). IR (neat): 2986, 1793, 1734, 1634, 1469, 1244, 1163, 1084, 1019, 904, $754 \mathrm{~cm}^{-1}$. HRMS Calcd for $\mathrm{C}_{11} \mathrm{H}_{12} \mathrm{O}_{5} \mathrm{Na}:[\mathrm{M}+\mathrm{Na}]^{+}, 240.0577$. Found: $m / z$ 240.0570.

(E)-(Isopropyl carbonic) pent-2-enoic anhydride (1h).<smiles>CCC=CC(=O)OC(=O)OC(C)C</smiles>

Colorless oil; $70 \%$ yield $(0.66 \mathrm{~g})$.

${ }^{1} \mathrm{H}$ NMR $\left(\mathrm{CDCl}_{3}\right) \delta 7.21(\mathrm{dt}, J=16.0,6.0 \mathrm{~Hz}, 1 \mathrm{H}), 5.82(\mathrm{~d}, J=16.0 \mathrm{~Hz}, 1 \mathrm{H}), 5.01$ (sept, $J=6.0 \mathrm{~Hz}, 1 \mathrm{H}), 2.28(\mathrm{~m}, 2 \mathrm{H}), 1.35(\mathrm{~d}, J=6.0 \mathrm{~Hz}, 6 \mathrm{H}), 1.09(\mathrm{t}, J=7.5 \mathrm{~Hz}, 3 \mathrm{H}) .{ }^{13} \mathrm{C}$ NMR $\left(\mathrm{CDCl}_{3}\right) \delta 160.8,156.0,148.8,118.3,74.1,25.7,21.5,11.8$. TLC: $\mathrm{R}_{\mathrm{f}} 0.37$ (hexane/EtOAc $=10: 1) . \quad$ IR (neat): 2984, 1802, 1743, 1648, 1465, 1378, 1233, 1169, 1080, 1027, $904 \mathrm{~cm}^{-1}$. HRMS Calcd for $\mathrm{C}_{9} \mathrm{H}_{14} \mathrm{O}_{4} \mathrm{Na}:[\mathrm{M}+\mathrm{Na}]^{+}, 209.0784$. Found: $\mathrm{m} / \mathrm{z}$ 209.0778.

(E)-(Isopropyl carbonic) 4-methylpent-2-enoic anhydride (1i).<smiles>CC(C)/C=C/C(=O)OC(=O)OC(C)C</smiles>

Colorless oil; $71 \%$ yield $(0.71 \mathrm{~g})$.

${ }^{1} \mathrm{H}$ NMR $\left(\mathrm{CDCl}_{3}\right) \delta 7.13(\mathrm{dd}, J=16.0,7.0 \mathrm{~Hz}, 1 \mathrm{H}), 6.78(\mathrm{dd}, J=16.0,1.5 \mathrm{~Hz}, 1 \mathrm{H})$, 5.00 (sept, $J=6.0 \mathrm{~Hz}, 1 \mathrm{H}), 2.50(\mathrm{~m}, 1 \mathrm{H}), 1.36(\mathrm{~d}, J=6.0 \mathrm{~Hz}, 6 \mathrm{H}), 1.08(\mathrm{~d}, J=7.0 \mathrm{~Hz}$, $6 \mathrm{H}) . \quad{ }^{13} \mathrm{C} \mathrm{NMR}\left(\mathrm{CDCl}_{3}\right) \delta 161.0,160.6,148.8,116.6,74.1,31.4,21.5,20.9$. TLC: $\mathrm{R}_{\mathrm{f}}$ 0.35 (hexane/EtOAc = 10:1). IR (neat): 2967, 1803, 1740, 1648, 1467, 1378, 1237, 1168, 1092, 902, $810 \mathrm{~cm}^{-1}$. HRMS Calcd for $\mathrm{C}_{10} \mathrm{H}_{16} \mathrm{O}_{4} \mathrm{Na}:[\mathrm{M}+\mathrm{Na}]^{+}, 223.0941$. Found: $m / z$ 223.0933.

\section{Procedure for preparation of $(Z)-\alpha, \beta$-unsaturated carbonic anhydride $1 a^{2}$}

A mixture of $\mathrm{K}_{2} \mathrm{PtCl}_{4}(103 \mathrm{mg}, 0.25 \mathrm{mmol})$ and $\mathrm{AgOTf}(129 \mathrm{mg}, 0.5 \mathrm{mmol})$ in trifluoroacetic acid $(5 \mathrm{~mL})$ was stirred at ambient temperature for $10 \mathrm{~min}$. Benzene $(5.3 \mathrm{~mL}, 60 \mathrm{mmol})$ and propionic acid $(0.61 \mathrm{~mL}, 10 \mathrm{mmol})$ were added to the mixture. Then, the mixture was stirred at the $40{ }^{\circ} \mathrm{C}$. After 3 days, the reaction mixture was poured into water $(20 \mathrm{~mL})$, neutralized with $\mathrm{NaHCO}_{3}$, and wasted with $\mathrm{Et}_{2} \mathrm{O}(30 \mathrm{~mL})$. Subsequently, the ethereal layers were extracted with aqueous $\mathrm{NaOH}(20 \mathrm{~mL} \times 3)$. 
The combined aqueous layers were washed with $\mathrm{Et}_{2} \mathrm{O}(20 \mathrm{~mL})$, acidified with $3 \mathrm{~N}$ aqueous $\mathrm{HCl}$, and extracted with $\mathrm{CH}_{2} \mathrm{Cl}_{2}(35 \mathrm{~mL})$. The organic layers were dried over $\mathrm{Na}_{2} \mathrm{SO}_{4}$ and concentrated in vacuo, affording $(Z)$-cinnamic acid $(810 \mathrm{mg}, 55 \%$ yield, $Z / E=25 / 1)$.

To a stirred solution of a $(Z)$-cinnamic acid $(480 \mathrm{mg}, 3.0 \mathrm{mmol})$ in $\mathrm{CH}_{2} \mathrm{Cl}_{2}(10 \mathrm{~mL})$ was slowly added $i$ - $\operatorname{Pr}_{2} \mathrm{EtN}(1.2 \mathrm{~mL}, 6.0 \mathrm{mmol})$ and isopropyl chloroformate $(0.45 \mathrm{~mL}$, $4.5 \mathrm{mmol}$ ) at $0{ }^{\circ} \mathrm{C}$, and the mixture was stirred at $0{ }^{\circ} \mathrm{C}$ for $30 \mathrm{~min} . \quad \mathrm{H}_{2} \mathrm{O}$ was added to the solution, and the mixture was extracted with $\mathrm{CH}_{2} \mathrm{Cl}_{2}$. The combined organic layers were washed with brine, dried over $\mathrm{Na}_{2} \mathrm{SO}_{4}$, and concentrated in vacuo. Purification by flash silica gel column chromatography using hexane/EtOAc $(\mathrm{v} / \mathrm{v}=10 / 1)$ as an eluent gave (isopropyl carbonic) (Z)-3-phenylacrylic anhydride (159 mg, 23\% yield, $Z / E=25 / 1)$.

(Z)-(Isopropyl carbonic) 3-phenylacrylic anhydride ((Z)-1a).<smiles>CC(C)OC(=O)OC(=O)/C=C\c1ccccc1</smiles>

Colorless oil.

${ }^{1} \mathrm{H}$ NMR $\left(\mathrm{CDCl}_{3}\right) \delta$ 7.70-7.67 (m, 2H), 7.39-7.37 (m, 3H), $7.16(\mathrm{~d}, J=12.5 \mathrm{~Hz}, 1 \mathrm{H})$, $5.93(\mathrm{~d}, J=12.5 \mathrm{~Hz}, 1 \mathrm{H}), 4.99$ (sept, $J=6.0 \mathrm{~Hz}, 1 \mathrm{H}), 1.34(\mathrm{~d}, J=6.0 \mathrm{~Hz}, 6 \mathrm{H}) .{ }^{13} \mathrm{C}$ $\operatorname{NMR}\left(\mathrm{CDCl}_{3}\right) \delta 159.9,148.8,148.6,133.8,130.3,130.1,128.2,116.4,74.2,21.4$. TLC: $R_{\mathrm{f}} 0.28$ (hexane/EtOAc = 10:1). IR (neat): 2985, 1798, 1745, 1621, 1251, 1058, 901, $697 \mathrm{~cm}^{-1}$. HRMS Calcd for $\mathrm{C}_{13} \mathrm{H}_{14} \mathrm{O}_{4} \mathrm{Na}$ : $[\mathrm{M}+\mathrm{Na}]^{+}, 257.0784$. Found: $\mathrm{m} / \mathrm{z}$ 257.0777 .

\section{Procedure for preparation of aminothiophenol $2 a^{3,4}$}

To the solution of dithiodianiline $(2.5 \mathrm{~g}, 10 \mathrm{mmol})$ in pridine $(30 \mathrm{~mL})$ was added $\mathrm{TsCl}$ (4.4 g, $23 \mathrm{mmol})$, and the mixture was stirred at ambient temperature for 3 days. Then, the mixture was quenched with $4 \mathrm{~N}$ aqueous $\mathrm{HCl}$ and extracted with EtOAc (20 $\mathrm{mL} \times 3)$. The combined organic layers were washed with brine, and dried over $\mathrm{Na}_{2} \mathrm{SO}_{4}$, and concentrated in vacuo. The crude product was used for the next step without further purification.

To

the

solution

of

$N, N$-(disulfanediylbis(2,1-phenylene))bis(4-methylbenzenesulfonamide) $\quad(5.5 \mathrm{~g}, \quad 10$ mmol) in a mixture of $\mathrm{H}_{2} \mathrm{O}$ and $\mathrm{THF}(\mathrm{v} / \mathrm{v}=1 / 1,30 \mathrm{~mL})$ was added triphenylphosphine $(5.5 \mathrm{~g}, 21 \mathrm{mmol})$, and the mixture was stirred until the starting material disappeared. 
The mixture was then extracted with $\mathrm{CH}_{2} \mathrm{Cl}_{2}(20 \mathrm{~mL} \times 3)$. The combined organic layers were washed with brine, dried over $\mathrm{Na}_{2} \mathrm{SO}_{4}$, and concentrated in vacuo. Purification by flash silica gel column chromatography using hexane/ $\mathrm{CH}_{2} \mathrm{Cl}_{2}(\mathrm{v} / \mathrm{v}=1 / 1)$ as an eluent gave $\mathrm{N}$-(2-mercaptophenyl)-4-methylbenzenesulfonamide $(5.1 \mathrm{~g}, 91 \%$ yield).

$N$-(2-Mercaptophenyl)-4-methylbenzenesulfonamide (2a): CAS RN [55423-98-8].<smiles>FC(F)(S)Nc1ccccc1S</smiles>

White solid

${ }^{1} \mathrm{H}$ NMR $\left(\mathrm{CDCl}_{3}\right) \delta 7.65(\mathrm{~m}, 2 \mathrm{H}), 7.54(\mathrm{~d}, J=8.0 \mathrm{~Hz}, 1 \mathrm{H}), 7.38(\mathrm{~d}, J=8.0 \mathrm{~Hz}, 1 \mathrm{H})$, $7.24(\mathrm{~m}, 3 \mathrm{H}), 7.02(\mathrm{t}, J=6.0 \mathrm{~Hz}, 1 \mathrm{H}), 2.80(\mathrm{~s}, 1 \mathrm{H}), 2.39(\mathrm{~s}, 3 \mathrm{H}) .{ }^{13} \mathrm{C} \mathrm{NMR}\left(\mathrm{CDCl}_{3}\right) \delta$ 144.2 , 137.5, 136.1, 135.1, 129.7, 129.3, 127.2, 125.5, 122.3, 119.7, 21.6. Мp. 72.8-73.8 ${ }^{\circ} \mathrm{C}$. TLC: $\quad \mathrm{R}_{\mathrm{f}} 0.15$ (hexane/ $\mathrm{CH}_{2} \mathrm{Cl}_{2}=1: 1$ ). HRMS Calcd for $\mathrm{C}_{13} \mathrm{H}_{13} \mathrm{NO}_{2} \mathrm{~S}_{2} \mathrm{Na}$ : [M+Na] $]^{+}$, 302.0280. Found: $m / z$ 302.0272.

\section{Procedure for preparation of aminothiophenol $2 b^{5}$}

To the solution of 4-chloro-2-mercaptoaniline (1.6 g, $10 \mathrm{mmol})$ and $\mathrm{NaI}(15 \mathrm{mg}, 0.1$ mmol) in EtOAc $(30 \mathrm{~mL})$ was added aqueous $\mathrm{H}_{2} \mathrm{O}_{2}(30 \mathrm{wt} \%, 1.1 \mathrm{~mL}, 10 \mathrm{mmol})$ at $0{ }^{\circ} \mathrm{C}$, and the mixture was stirred at $0{ }^{\circ} \mathrm{C}$ for $30 \mathrm{~min}$. The mixture was subsequently quenched with saturated aqueous $\mathrm{Na}_{2} \mathrm{~S}_{2} \mathrm{O}_{3}$ and extracted with EtOAc $(15 \mathrm{~mL} \times 3)$. The combined organic layers were washed with brine, dried over $\mathrm{Na}_{2} \mathrm{SO}_{4}$, and concentrated in vacuo. The crude product was used for the next step without further purification.

To the solution of 6,6'-disulfanediylbis(3-chloroaniline) (1.6 g, $5 \mathrm{mmol}$ ) in pridine $(20 \mathrm{~mL})$ was added $\mathrm{TsCl}(3.8 \mathrm{~g}, 20 \mathrm{mmol})$, and the mixture was stirred at ambient temperature for 3 days. Then, the mixture was quenched with aqueous $4 \mathrm{~N} \mathrm{HCl}$ and extracted with EtOAc $(20 \mathrm{~mL} \times 3)$. The combined organic layers were washed with brine, dried over $\mathrm{Na}_{2} \mathrm{SO}_{4}$, and concentrated in vacuo. The crude product was used for the next step without further purification.

To

the

solution

of

$N, N^{\prime}$-(disulfanediylbis(3-chloro-6,1-phenylene))bis(4-methylbenzenesulfonamide)

$\mathrm{g}, 5 \mathrm{mmol})$ in a mixture of $\mathrm{H}_{2} \mathrm{O}$ and $\mathrm{THF}(\mathrm{v} / \mathrm{v}=1 / 1,20 \mathrm{~mL})$ was added triphenylphosphine $(2.6 \mathrm{~g}, 10 \mathrm{mmol})$, and the mixture was stirred until the starting 
material disappeared. The mixture was then extracted with $\mathrm{CH}_{2} \mathrm{Cl}_{2}(20 \mathrm{~mL} \times 3)$. The combined organic layers were washed with brine, dried over $\mathrm{Na}_{2} \mathrm{SO}_{4}$, and concentrated in vacuo. Purification by flash silica gel column chromatography using hexane $/ \mathrm{CH}_{2} \mathrm{Cl}_{2} \quad(\mathrm{v} / \mathrm{v} \quad=\quad 1 / 1)$ as an eluent gave $\mathrm{N}$-(5-chloro-2-mercaptophenyl)-4-methylbenzenesulfonamide.

$N$-(5-Chloro-2-mercaptophenyl)-4-methylbenzenesulfonamide (2b).<smiles>Sc1ccc(Cl)cc1N[Sn]</smiles>

White solid; $37 \%$ yield (1.2 g: for 3 steps).

${ }^{1} \mathrm{H}$ NMR $\left(\mathrm{CDCl}_{3}\right) \delta$ 7.70-7.68 (m, 2H), $7.59(\mathrm{~d}, J=2.5 \mathrm{~Hz}, 1 \mathrm{H}), 7.35$ (brs, 1H), 7.33 (d, $J=8.5 \mathrm{~Hz}, 1 \mathrm{H}), 7.27(\mathrm{~m}, 2 \mathrm{H}), 6.99(\mathrm{dd}, J=8.5,2.5 \mathrm{~Hz}, 1 \mathrm{H}), 2.77$ (s, 1H), 2.40 (s, 3H). ${ }^{13} \mathrm{C} \mathrm{NMR}\left(\mathrm{CDCl}_{3}\right) \delta 144.5,138.9,136.2,135.8,135.4,129.9,127.2,125.3,121.4,116.9$, 21.6. IR (KBr): 3268, 2577, 1566, 1476, 1377, 1332, 1166, 1090, 807, $675 \mathrm{~cm}^{-1}$. Mp. 91.9-92.9 ${ }^{\circ} \mathrm{C}$. TLC: $\mathrm{R}_{\mathrm{f}} \quad 0.25$ (hexane/EtOAc $=1: 1$ ). HRMS Calcd for $\mathrm{C}_{13} \mathrm{H}_{12} \mathrm{ClNO}_{2} \mathrm{~S}_{2} \mathrm{Na}:[\mathrm{M}+\mathrm{Na}]^{+}, 335.9890$. Found: $m / z 335.9884$.

\section{General procedure for preparation of aminothiols $2 c-e^{6}$}

A solution of 2-methylbenzothiazole derivative $(10 \mathrm{mmol})$ in a mixture of aqueous $50 \% \mathrm{NaOH}$ and ethylene glycol $(\mathrm{v} / \mathrm{v}=1 / 1,20 \mathrm{~mL})$ was refluxed under argon flux until the starting material disappeared. The mixture was then poured into ice-water, acidified to $\mathrm{pH}=3$ with $3 \mathrm{~N}$ aqueous $\mathrm{HCl}$, and extracted several times with $\mathrm{CH}_{2} \mathrm{Cl}_{2}$. The combined organic layers were washed with brine, dried over $\mathrm{Na}_{2} \mathrm{SO}_{4}$, and concentrated in vacuo. Purification by flash silica gel column chromatography using hexane/EtOAc ( $/ \mathrm{v}=2 / 1$ to $1 / 1)$ as an eluent gave the corresponding aminothiophenols (64-97\% yield).

To the solution of the obtained aminothiophenol derivative (1.0 equiv) and $\mathrm{NaI}$ (5 mol \%) in EtOAc $(0.5 \mathrm{M})$ was added aqueous $\mathrm{H}_{2} \mathrm{O}_{2}\left(30 \mathrm{wt} \%, 1.0\right.$ equiv) at $0{ }^{\circ} \mathrm{C}$, and the mixture was stirred at $0{ }^{\circ} \mathrm{C}$ for $30 \mathrm{~min}$. The reaction was subsequently quenched by saturated aqueous $\mathrm{Na}_{2} \mathrm{~S}_{2} \mathrm{O}_{3}$, and the mixture was extracted with EtOAc $(15 \mathrm{~mL} \times 3)$. The combined organic layers were washed with brine, dried over $\mathrm{Na}_{2} \mathrm{SO}_{4}$, and concentrated in vacuo. The crude product was used for the next step without further purification.

To the solution of 6,6'-disulfanediylbis(3-chloroaniline) (1.0 equiv) in pridine $(0.2$ 
M) was added $\mathrm{TsCl}$ (2.2 equiv), and the mixture was stirred at ambient temperature for 3 days. Then, the reaction was quenched with $4 \mathrm{~N}$ aqueous $\mathrm{HCl}$, and the mixture was extracted with EtOAc $(20 \mathrm{~mL} \times 3)$. The combined organic layers were washed with brine, dried over $\mathrm{Na}_{2} \mathrm{SO}_{4}$, and concentrated in vacuo. The crude product was used for the next step without further purification.

To the solution of $N, N^{\prime}$-(disulfanediylbis(3-chloro-6,1-phenylene))bis(4-methylbenzenesulfonamide) (1.0 equiv) in a mixture of $\mathrm{H}_{2} \mathrm{O}$ and THF ( $\left.\mathrm{v} / \mathrm{v}=1 / 1,0.05 \mathrm{M}\right)$ was added triphenylphosphine (2.1 equiv), and the mixture was stirred until the starting material disappeared. The mixture was then extracted with $\mathrm{CH}_{2} \mathrm{Cl}_{2}(20 \mathrm{~mL} \times 3)$. The combined organic layers were washed with brine, dried over $\mathrm{Na}_{2} \mathrm{SO}_{4}$, and concentrated in vacuo. Purification by flash silica gel column chromatography using hexane $/ \mathrm{CH}_{2} \mathrm{Cl}_{2}(\mathrm{v} / \mathrm{v}=1 / 1)$ as an eluent gave the corresponding $N$-(2-mercaptophenyl)-4-methylbenzenesulfonamides.

\section{$N$-(2-Mercapto-5-methylphenyl)-4-methylbenzenesulfonamide (2c).}<smiles>Cc1ccc(S)c(N)c1</smiles>

Yellow solid; $13 \%$ yield (0.75 g: for 4 steps).

${ }^{1} \mathrm{H} \mathrm{NMR}\left(\mathrm{CDCl}_{3}\right) \delta 7.64(\mathrm{~d}, J=8.5 \mathrm{~Hz}, 2 \mathrm{H}), 7.41(\mathrm{~d}, J=2.0 \mathrm{~Hz}, 1 \mathrm{H}), 7.30$ (brs, $\left.1 \mathrm{H}\right)$, $7.27(\mathrm{~d}, J=8.5 \mathrm{~Hz}, 1 \mathrm{H}), 7.22(\mathrm{~m}, 2 \mathrm{H}), 6.83(\mathrm{dd}, J=8.0,2.0 \mathrm{~Hz}, 1 \mathrm{H}), 2.62$ (brs, $1 \mathrm{H})$, $2.39(\mathrm{~s}, 3 \mathrm{H}), 2.31(\mathrm{~s}, 3 \mathrm{H}) .{ }^{13} \mathrm{C} \mathrm{NMR}\left(\mathrm{CDCl}_{3}\right) \delta 144.1,140.1,137.7,136.2,135.3$, 129.7, 127.2, 126.3, 122.7, 115.3, 21.6, 21.4. IR (KBr): 3236, 2919, 2569, 1598, 1488, 1379, 1331, 1173, 1164, 899, 815, $679 \mathrm{~cm}^{-1}$. Mp. 71.0-72.0 ${ }^{\circ} \mathrm{C}$. TLC: $\mathrm{R}_{\mathrm{f}} 0.21$ (hexane/EtOAc $=2: 1$ ). HRMS Calcd for $\mathrm{C}_{14} \mathrm{H}_{15} \mathrm{NO}_{2} \mathrm{~S}_{2} \mathrm{Na}:[\mathrm{M}+\mathrm{Na}]^{+}, 316.0436$. Found: $m / z 316.0429$.

$N$-(2-Mercapto-5-methoxyphenyl)-4-methylbenzenesulfonamide (2d).<smiles>COc1ccc(S)c(N)c1</smiles>

Yellow solid; $14 \%$ yield (0.42 g: for 4 steps).

${ }^{1} \mathrm{H} \mathrm{NMR}\left(\mathrm{CDCl}_{3}\right) \delta 7.68(\mathrm{~d}, J=8.0 \mathrm{~Hz}, 2 \mathrm{H}), 7.56$ (brs, $\left.1 \mathrm{H}\right), 7.33(\mathrm{~d}, J=8.5 \mathrm{~Hz}, 1 \mathrm{H})$, $7.23(\mathrm{~d}, J=8.0 \mathrm{~Hz}, 2 \mathrm{H}), 7.18(\mathrm{~d}, J=3.0 \mathrm{~Hz}, 1 \mathrm{H}), 6.56(\mathrm{dd}, J=8.5,3.0 \mathrm{~Hz}, 1 \mathrm{H}), 3.78(\mathrm{~s}$, $3 \mathrm{H}), 2.58$ (brs, $1 \mathrm{H}), 2.38(\mathrm{~s}, 3 \mathrm{H}) .{ }^{13} \mathrm{C} \mathrm{NMR}\left(\mathrm{CDCl}_{3}\right) \delta 160.9,144.2,139.8,137.2$, 136.0, 129.7, 127.2, 111.3, 107.5, 106.3, 55.5, 21.6. IR (KBr): 3250, 2948, 2545, 1600, 
1571, 1490, 1398, 1335, 1295, 1164, 891, $673 \mathrm{~cm}^{-1}$. Mp. 75.6-76.2 ${ }^{\circ} \mathrm{C} . \quad$ TLC: $\mathrm{R}_{\mathrm{f}}$ 0.24 (hexane/EtOAc $=1: 1)$. HRMS Calcd for $\mathrm{C}_{14} \mathrm{H}_{15} \mathrm{NO}_{3} \mathrm{~S}_{2} \mathrm{Na}:[\mathrm{M}+\mathrm{Na}]^{+}, 332.0386$. Found: $m / z 332.0374$.

$N$-(5-Bromo-2-mercaptophenyl)-4-methylbenzenesulfonamide (2e).<smiles>Sc1ccc(Br)cc1N[AsH3]</smiles>

White solid; $25 \%$ yield (0.88 g: for 4 steps).

${ }^{1} \mathrm{H} \mathrm{NMR}\left(\mathrm{CDCl}_{3}\right) \delta$ 7.71-7.68 (m, 3H), 7.36 (brs, 1H), 7.30-7.24 (m, 3H), $7.15(\mathrm{~m}, 1 \mathrm{H})$, 2.83 (brs, $1 \mathrm{H}), 2.40(\mathrm{~s}, 3 \mathrm{H}) .{ }^{13} \mathrm{C} \mathrm{NMR}\left(\mathrm{CDCl}_{3}\right) \delta 144.5,138.6,136.0,135.7,129.8$, 128.3, 127.1, 124.6, 122.8, 118.3, 21.6. IR (KBr): 3235, 2551, 1573, 1468, 1378, 1336, 1164, 1089, 922, 808, 733, $679 \mathrm{~cm}^{-1}$. Mp. 93.8-94.3 ${ }^{\circ} \mathrm{C}$. TLC: $\mathrm{R}_{\mathrm{f}} 0.25$ (hexane/EtOAc $=2: 1$ ). HRMS Calcd for $\mathrm{C}_{13} \mathrm{H}_{12} \mathrm{BrNO}_{2} \mathrm{~S}_{2} \mathrm{Na}:[\mathrm{M}+\mathrm{Na}]^{+}, 355.9420$. Found: $m / z 355.9425$. 
Table S1. Screening of Catalysts ${ }^{\mathrm{a}}$

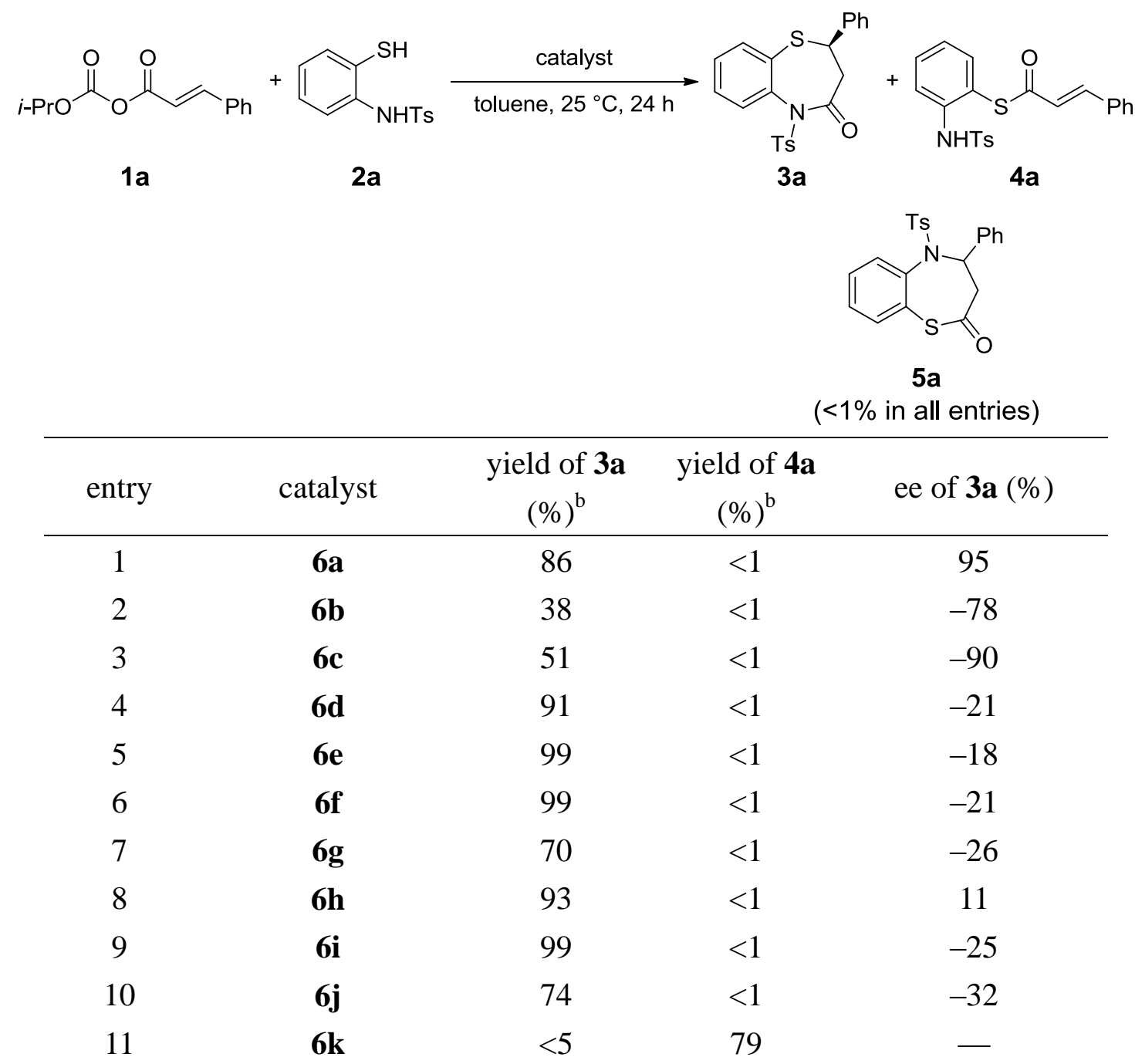

${ }^{\mathrm{a}}$ Reactions were run using 1a $(0.15 \mathrm{mmol}), \mathbf{2 a}(0.15 \mathrm{mmol})$, and the catalyst $(0.015 \mathrm{mmol})$ in toluene $(0.3 \mathrm{~mL}) .{ }^{\mathrm{b}}$ Isolated yields. 
(Table S1)

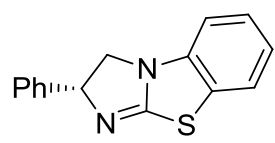

$6 a$<smiles>C=CC1C2CCN1C([C@H](NC(=S)Nc1cc(C(F)(F)F)cc(C(F)(F)F)c1)c1ccnc3ccc(OC)cc13)C2</smiles>

$6 e$<smiles>c1ccc([C@@H]2CN3CCSC3=N2)cc1</smiles>

6b<smiles>CC1N=C2Sc3ccccc3N2[C@@H]1c1ccccc1</smiles>

$6 c$<smiles>CN(C)[C@@H]1CCCC[C@H]1NC(=S)Nc1cc(C(F)(F)F)cc(C(F)(F)F)c1</smiles>

6d<smiles>C=CC1CC2CCN1CC2C(NC(=S)Nc1cc(C(F)(F)F)cc(C(F)(F)F)c1)c1ccnc2ccccc12</smiles><smiles>C=CC1CC2CCN1CC2[C@H](O)c1ccnc2ccccc12</smiles><smiles>COc1ccc2nccc([C@@H](NC(=S)Nc3cc(C(F)(F)F)cc(C(F)(F)F)c3)[C@@H]3C4C=CC5CC4NN3C5)c2c1</smiles>

$6 \mathrm{~g}$<smiles>C=CC1C2CCN1CC2[C@H](NC(=O)C(NC(=S)Nc1cc(C(F)(F)F)cc(C(F)(F)F)c1)C(C)C)c1ccnc2ccc(OC)cc12</smiles>

$6 \mathbf{i}$<smiles>CN(C)[C@H](c1ccccc1)C(NC(=S)Nc1cc(C(F)(F)F)cc(C(F)(F)F)c1)c1ccccc1</smiles>

6j<smiles>Cc1ccc(-c2ccccc2)n1C1CCCC[C@H]1NC(=S)N[C@H](C(=O)N(CC(C)(C)C)CC(C)(C)C)C(C)(C)C</smiles>

6k 
Table S2. The Effects of Concentration ${ }^{\mathrm{a}}$

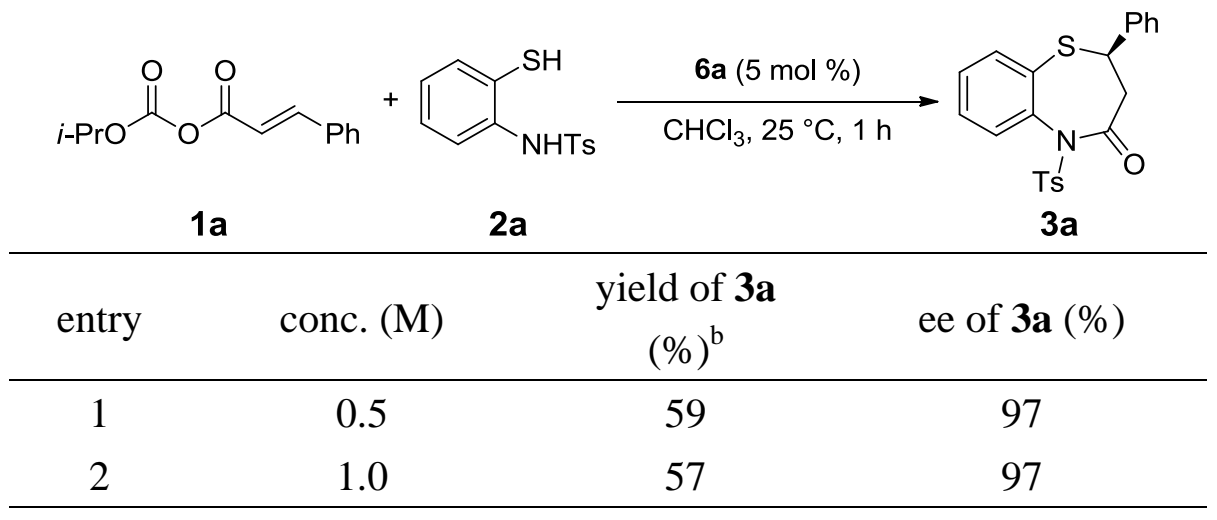

${ }^{\mathrm{a}}$ Reactions were run using $\mathbf{1 a}(0.15 \mathrm{mmol}), \mathbf{2 a}(0.15 \mathrm{mmol})$, and the catalyst $(0.0075 \mathrm{mmol})$ in $\mathrm{CHCl}_{3}$. $\quad{ }^{\mathrm{b}}$ Isolated yields. 
Scheme S1. HRMS Analysis of the Solution of 1a in the Presence of a Stoichiometric Amount of $\mathbf{6 a}$<smiles>CC(C)OC(=O)OC(=O)/C=C/c1ccccc1</smiles>

$1 \mathrm{a}$ $(0.06 \mathrm{mmol})$

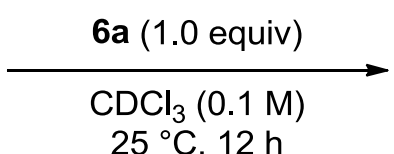

$25^{\circ} \mathrm{C}, 12 \mathrm{~h}$

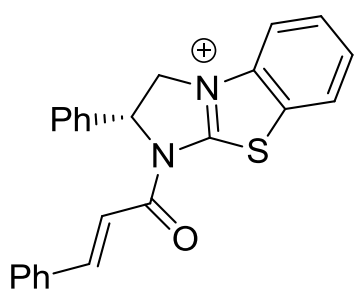

acylammonium intermediate HRMS Found: $m / z$ 383.1213.

(Calcd: $m / z$ 383.1202.) 
(a) $\alpha, \beta$-unsaturated substrate 1a

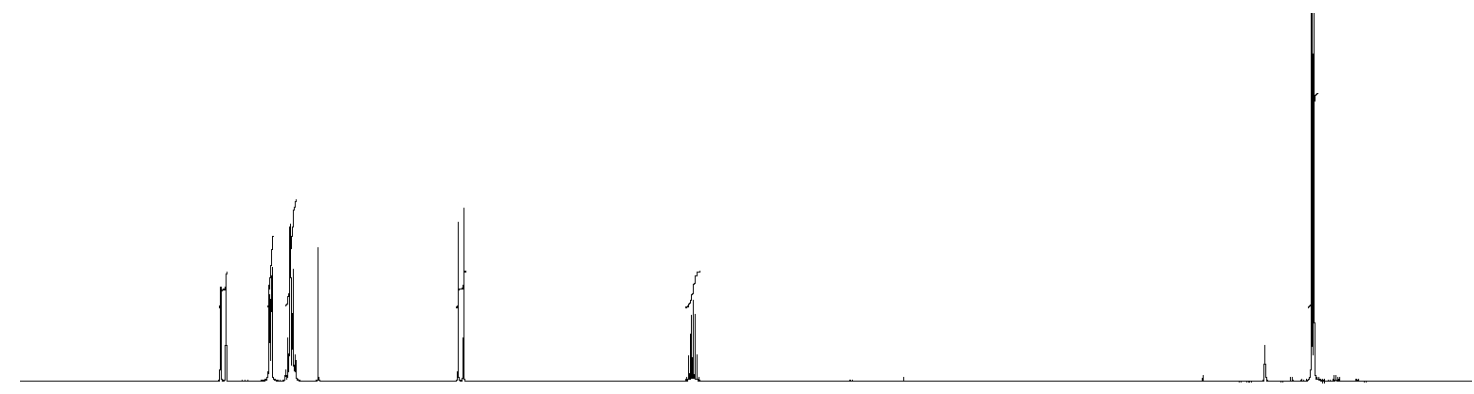

(b) catalyst $\mathbf{6 a}$

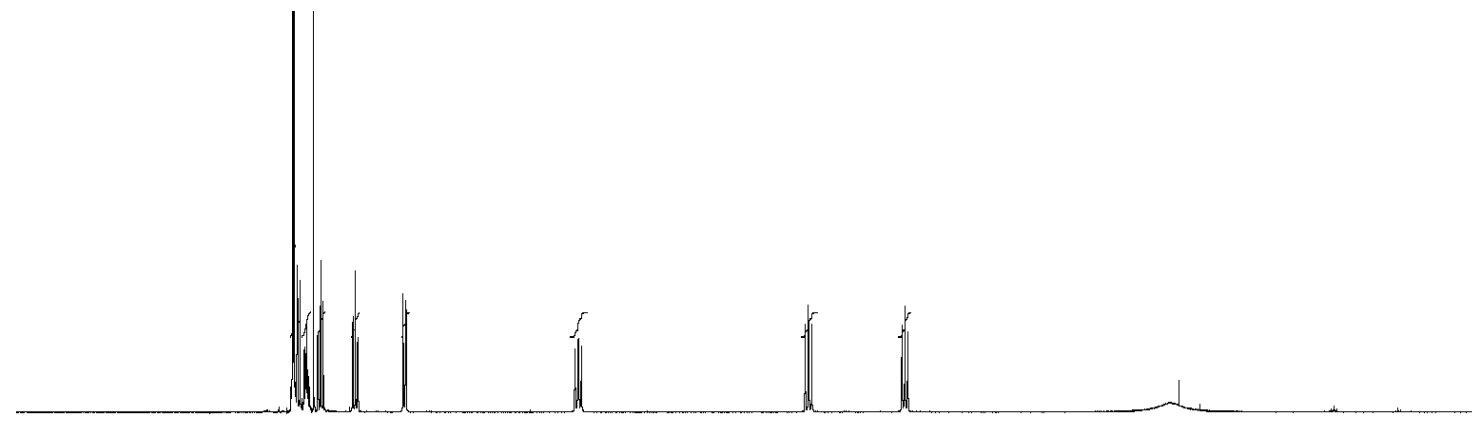

(c) mixture of $1 \mathbf{a}$ and $\mathbf{6 a}(1: 1)$

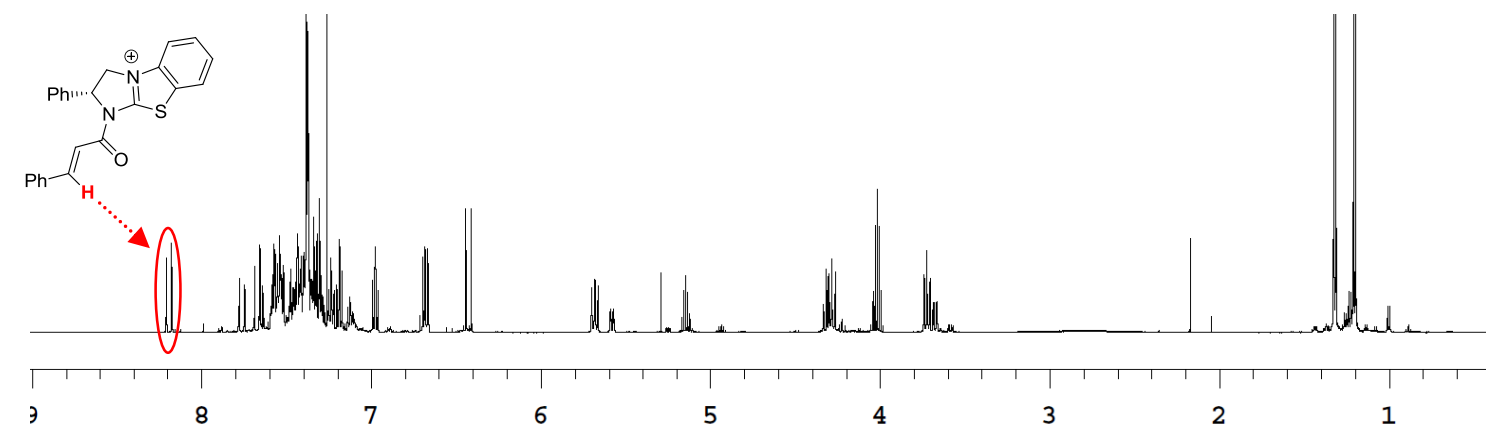

Figure S1. Independent ${ }^{1} \mathrm{H}$ NMR spectra of (a) $\alpha, \beta$-unsaturated substrate 1a and (b) catalyst $\mathbf{6 a}$ and the spectra of (c) their 1:1 mixture $\left(0.1 \mathrm{M}, \mathrm{CDCl}_{3}\right.$, ambient temperature). 
(a) $\alpha, \beta$-unsaturated substrate $\mathbf{1 a}$

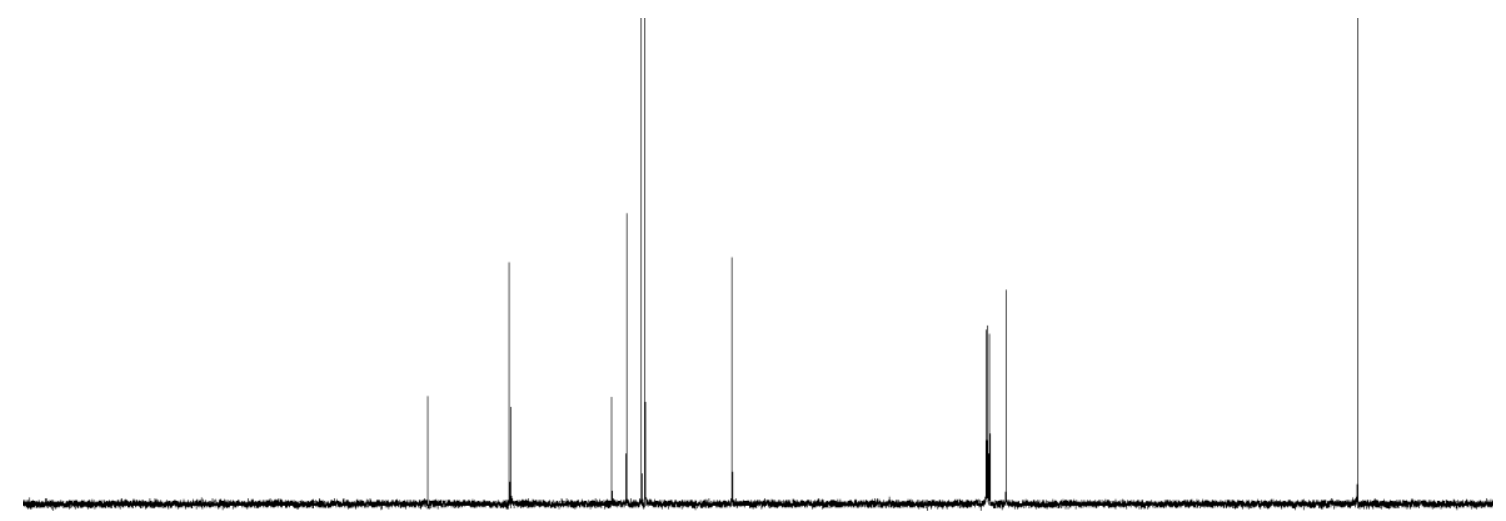

(b) catalyst $\mathbf{6 a}$

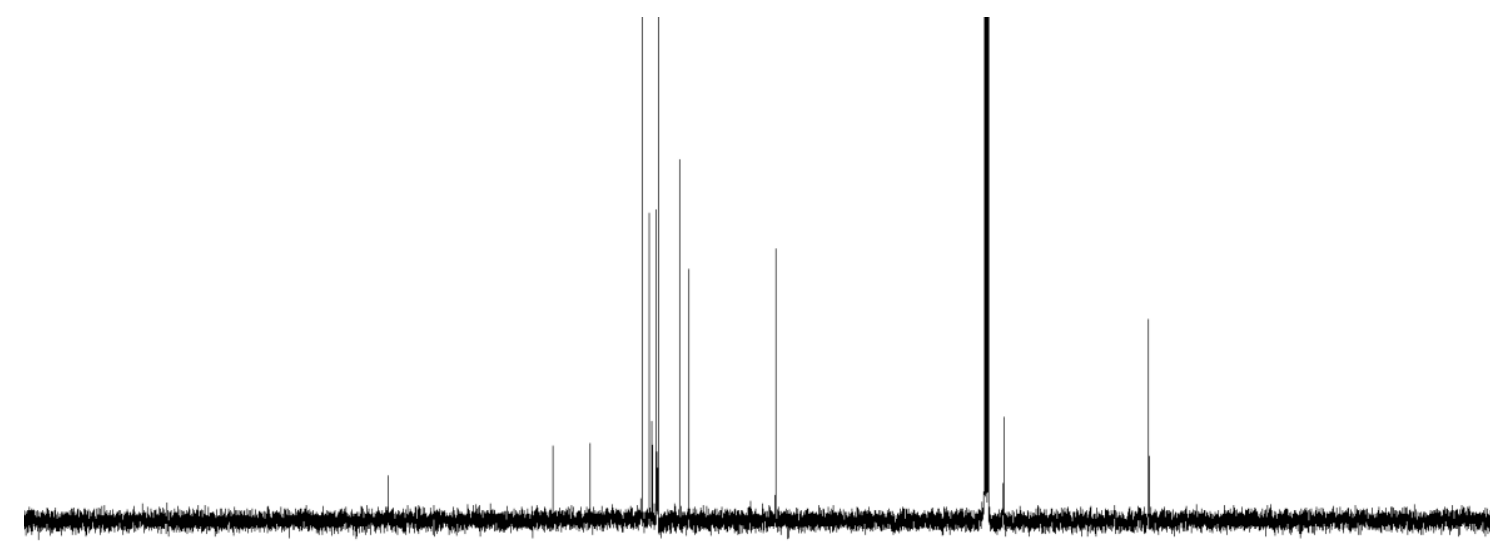

(c) mixture of 1a and 6a (1:1)

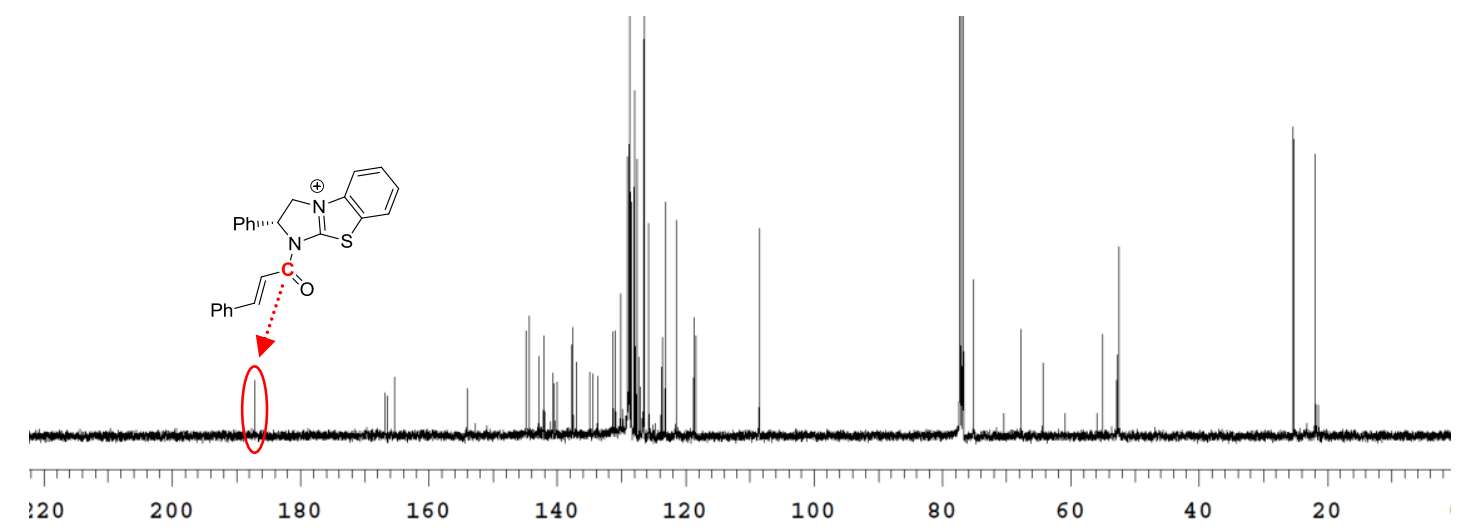

Figure S2. Independent ${ }^{13} \mathrm{C}$ NMR spectra of (a) $\alpha, \beta$-unsaturated substrate 1a and (b) catalyst $\mathbf{6 a}$ and the spectra of (c) their $1: 1$ mixture $\left(0.1 \mathrm{M}, \mathrm{CDCl}_{3}\right.$, ambient temperature). 
Scheme S2. Reaction of the Acylammonium Intermediate Prepared by Stoichiometric Reaction

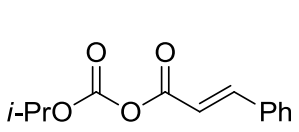

$1 \mathrm{a}$

$(0.15 \mathrm{mmol})$

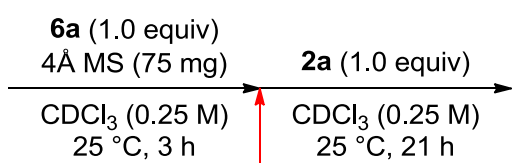

$25{ }^{\circ} \mathrm{C}, 3 \mathrm{~h}$

$25{ }^{\circ} \mathrm{C}, 21 \mathrm{~h}$

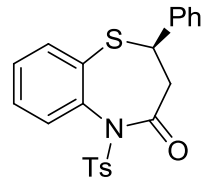

$3 \mathbf{a}$

$61 \%, 96 \%$ ee<smiles>O=C(/C=C/c1ccccc1)OC(I)I</smiles>

16

$25 \%$

The formation of the acylammonium intermediate was confirmed by the NMR analyses (Figures 1 and 2). 
Scheme S3. Reactions of Benzenethiol 9 with $(E)$ - and (Z)-1a in the Presence of $\mathbf{6 a}$

a

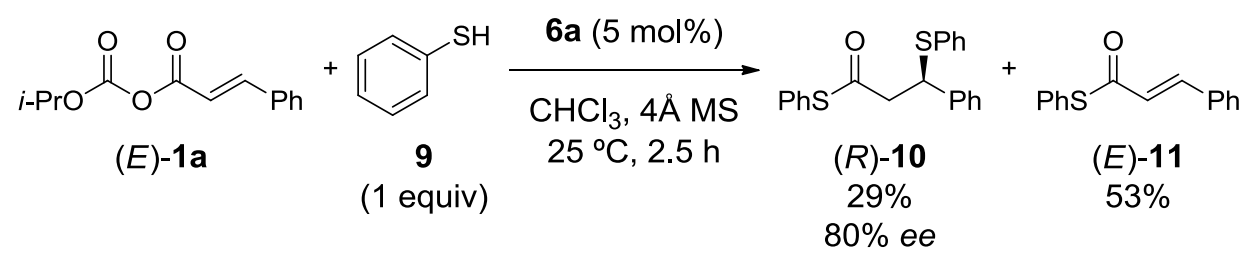

b

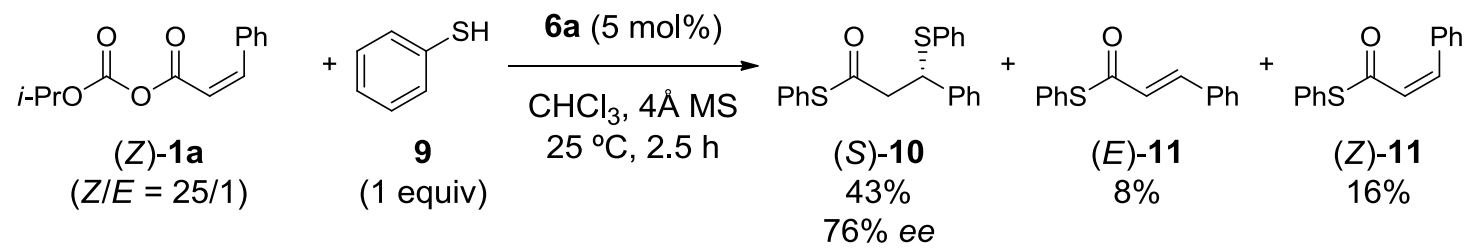

\section{Procedure for ractions of benzenethiol and $\alpha, \beta$-unsaturated carbonic anhydrides}

To a 5-mL vial were added sequentially $\alpha, \beta$-unsaturated carbonic anhydride $\mathbf{1 a}(0.15$ $\mathrm{mmol}), \mathrm{CHCl}_{3}(0.3 \mathrm{~mL}), 4 \AA \mathrm{MS}(75 \mathrm{mg})$, benzotetramisole catalyst $6 \mathbf{a}(0.0075 \mathrm{mmol})$, and benzenethiol $9(0.15 \mathrm{mmol})$. The mixture was stirred in an oil bath maintained at $25{ }^{\circ} \mathrm{C}$ for $2.5 \mathrm{~h}$. The reaction mixture was subsequently diluted with hexane/EtOAc $(\mathrm{v} / \mathrm{v}=20 / 1)$, passed through a short silica gel pad to remove $\mathbf{6 a}$, and concentrated in vacuo. Purification of the reaction mixture by flash silica gel column chromatography using hexane/EtOAc $(\mathrm{v} / \mathrm{v}=10 / 1)$ as an eluent afforded the mixture of $\mathbf{1 0}$ and $\mathbf{1 1}$.

The absolute configuration of $\mathbf{1 0}$ obtained in the reaction of Scheme S2 (a) was assigned as $(R)$, and that of Scheme $\mathrm{S} 2(\mathrm{~b})$ was assigned as $(S)$ by comparing the optical rotation with the literature value.

10 of Scheme S2 (a): $[\alpha]_{\mathrm{D}}{ }^{18}+19.9\left(c 3.40, \mathrm{CH}_{2} \mathrm{Cl}_{2}\right)$

10 of Scheme S2 (b): $[\alpha]_{\mathrm{D}}{ }^{18}-21.6\left(c 3.41, \mathrm{CH}_{2} \mathrm{Cl}_{2}\right)$

[lit. ${ }^{7} 73 \%$ ee, $(R)$-S-phenyl 3-phenyl-3-(phenylthio)propanethioate: $[\alpha]_{\mathrm{D}}{ }^{25}+43.5(c 3.47$, $\left.\left.\mathrm{CH}_{2} \mathrm{Cl}_{2}\right)\right]$. 
Scheme S4. Net [4+3] Formal Cycloaddition Reaction Using $\alpha, \beta$-Unsaturatred Carboxylic Acid Chloride<smiles>O=C(Cl)/C=C/Pc1ccccc1</smiles>

12

$(0.15 \mathrm{mmol})$<smiles>Sc1ccccc1N[I-]</smiles>

$2 a$

(1.0 equiv)

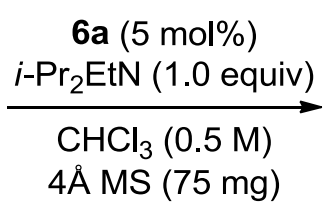

$25^{\circ} \mathrm{C}, 24 \mathrm{~h}$<smiles>O=C1C[C@H](c2ccccc2)Sc2ccccc2N1[As]</smiles>

$3 a$

$91 \%$ $88 \%$ ee 
Scheme S5. Net [4+3] Formal Cycloaddition Reaction through the In-Situ Generation of Carbonic Anhydride Uging $\alpha, \beta$-Unsaturatred Carboxylic Acid<smiles>O=C(O)/C=C/c1ccccc1</smiles>

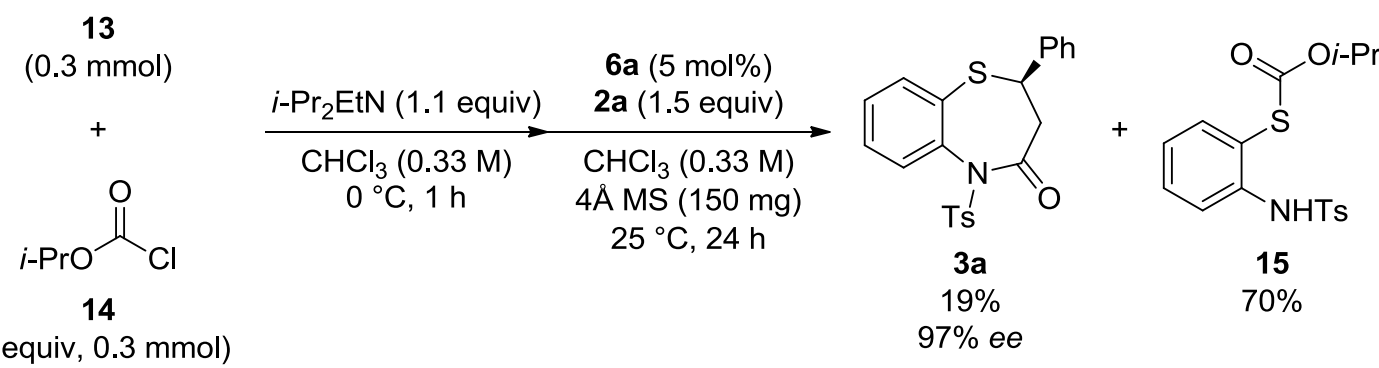




\section{Characterization Data of Products}

$(R)$-2-Phenyl-5-tosyl-2,3-dihydrobenzo[b][1,4]thiazepin-4(5H)-one (3a).<smiles>[3H]N1C(=O)CC(c2ccccc2)Sc2ccccc21</smiles>

From (E)-1a: $95 \%$ yield (58.1 mg), 97\% ee.

From (Z)-1a: $75 \%$ yield (46.1 mg), 97\% ee.

White solid. $\quad[\alpha]_{\mathrm{D}}{ }^{18}-246.7\left(c 0.68, \mathrm{CH}_{2} \mathrm{Cl}_{2}\right) . \quad{ }^{1} \mathrm{H} \mathrm{NMR}\left(\mathrm{CDCl}_{3}\right) \delta 7.91(\mathrm{~d}, J=8.5 \mathrm{~Hz}$, 2H), $7.80(\mathrm{~d}, J=8.0 \mathrm{~Hz}, 1 \mathrm{H}), 7.64-7.60(\mathrm{~m}, 2 \mathrm{H}), 7.49(\mathrm{~d}, J=7.5 \mathrm{~Hz}, 1 \mathrm{H}), 7.34$ (d, $J=$ $8.5 \mathrm{~Hz}, 2 \mathrm{H}), 7.25-7.23(\mathrm{~m}, 3 \mathrm{H}), 6.93(\mathrm{~d}, J=7.5 \mathrm{~Hz}, 1 \mathrm{H}), 6.92(\mathrm{~d}, J=5.5 \mathrm{~Hz}, 1 \mathrm{H}), 4.43$ (dd, $J=11.0,7.0 \mathrm{~Hz}, 1 \mathrm{H}), 2.76(\mathrm{~d}, J=11.0 \mathrm{~Hz}, 1 \mathrm{H}), 2.76(\mathrm{~d}, J=7.0 \mathrm{~Hz}, 1 \mathrm{H}), 2.48$ (s, $3 \mathrm{H}) . \quad{ }^{13} \mathrm{C} \mathrm{NMR}\left(\mathrm{CDCl}_{3}\right) \delta 169.2,145.2,142.2,140.3,136.9,135.5,130.5,130.2$, 130.0, 129.6, 129.1, 128.8, 127.9, 127.5, 125.9, 50.5, 42.4, 21.8. Mp. 144.5-145.5 ${ }^{\circ} \mathrm{C}$. TLC: $R_{\mathrm{f}} 0.26$ (hexane/EtOAc = 3:1). IR $(\mathrm{KBr}): 2363,1712,1366,1286,1172,1129$, 1086, 924, 843, $765 \mathrm{~cm}^{-1}$. HRMS Calcd for $\mathrm{C}_{22} \mathrm{H}_{20} \mathrm{NO}_{3} \mathrm{~S}_{2}$ : $[\mathrm{M}+\mathrm{H}]^{+}, 410.0879$. Found: $m / z$ 410.0877. HPLC (Daicel Chiralpak IF, hexane $/ i$-PrOH $=80 / 20$, flow rate $=2.0$ $\left.\mathrm{mL} / \mathrm{min}, \lambda=254 \mathrm{~nm}, 30^{\circ} \mathrm{C}\right): t_{\text {minor }}=15.5 \mathrm{~min}, t_{\text {major }}=18.6 \mathrm{~min}$.

7-Methyl-2-phenyl-5-tosyl-2,3-dihydrobenzo[b][1,4]thiazepin-4(5H)-one (3b).<smiles>Cc1ccc2c(c1)N([13S])C(=O)CC(c1ccccc1)S2</smiles>

Yield: 94\% (59.9 mg), 97\% ee, white solid. $\quad[\alpha]_{\mathrm{D}}{ }^{18}-260.9\left(c 0.92, \mathrm{CH}_{2} \mathrm{Cl}_{2}\right) . \quad{ }^{1} \mathrm{H} \mathrm{NMR}$ $\left(\mathrm{CDCl}_{3}\right) \delta 7.89(\mathrm{~d}, J=8.0 \mathrm{~Hz}, 2 \mathrm{H}), 7.59(\mathrm{~m}, 1 \mathrm{H}), 7.46(\mathrm{~d}, J=8.0 \mathrm{~Hz}, 1 \mathrm{H}), 7.32(\mathrm{~d}, J=$ $8.0 \mathrm{~Hz}, 2 \mathrm{H}), 7.27(\mathrm{~m}, 2 \mathrm{H}), 7.22-7.21(\mathrm{~m}, 2 \mathrm{H}), 6.92(\mathrm{~m}, 2 \mathrm{H}), 4.38(\mathrm{dd}, J=12.0,6.5 \mathrm{~Hz}$, $1 \mathrm{H}), 2.73(\mathrm{~d}, J=12.0 \mathrm{~Hz}, 1 \mathrm{H}), 2.72(\mathrm{~d}, J=6.5 \mathrm{~Hz}, 1 \mathrm{H}), 2.51(\mathrm{~s}, 3 \mathrm{H}), 2.46(\mathrm{~s}, 3 \mathrm{H}) .{ }^{13} \mathrm{C}$ NMR $\left(\mathrm{CDCl}_{3}\right) \delta 169.4,145.1,142.6,140.9,140.0,136.6,131.2,130.8,130.5,129.6$, 129.0, 128.8, 127.8, 125.9, 123.9, 50.6, 42.5, 21.8, 21.3. Mp. 150.8-151.8 ${ }^{\circ} \mathrm{C} . \quad$ TLC: $\mathrm{R}_{\mathrm{f}} 0.36$ (hexane/EtOAc = 3:1). IR $(\mathrm{KBr}):$ 3029, 2344, 1710, 1599, 1362, 1169, 1086, $829,683 \mathrm{~cm}^{-1}$. HRMS Calcd for $\mathrm{C}_{23} \mathrm{H}_{21} \mathrm{NO}_{3} \mathrm{~S}_{2} \mathrm{Na}$ : $[\mathrm{M}+\mathrm{Na}]^{+}, 446.0855$. Found: $\mathrm{m} / z$ 446.0848. HPLC (Daicel Chiralpak IF, hexane $/ i$-PrOH $=80 / 20$, flow rate $=2.0$ $\left.\mathrm{mL} / \mathrm{min}, \lambda=254 \mathrm{~nm}, 30{ }^{\circ} \mathrm{C}\right): t_{\text {minor }}=8.5 \mathrm{~min}, t_{\text {major }}=10.5 \mathrm{~min}$. 
7-Methoxy-2-phenyl-5-tosyl-2,3-dihydrobenzo[b][1,4]thiazepin-4(5H)-one (3c).<smiles>COc1ccc2c(c1)N([13CH3])C(=O)C[C@@H](c1ccccc1)S2</smiles>

Yield: 79\% (52.0 mg), 97\% ee, white solid. $\quad[\alpha]_{\mathrm{D}}{ }^{18}-278.1\left(c 0.80, \mathrm{CH}_{2} \mathrm{Cl}_{2}\right) . \quad{ }^{1} \mathrm{H} \mathrm{NMR}$ $\left(\mathrm{CDCl}_{3}\right) \delta 7.88(\mathrm{~d}, J=8.5 \mathrm{~Hz}, 2 \mathrm{H}), 7.45(\mathrm{~d}, J=8.5 \mathrm{~Hz}, 1 \mathrm{H}), 7.33-7.31(\mathrm{~m}, 3 \mathrm{H})$, $7.24-7.21(\mathrm{~m}, 3 \mathrm{H}), 7.00(\mathrm{dd}, J=8.5,3.0 \mathrm{~Hz}, 1 \mathrm{H}), 6.92-6.89(\mathrm{~m}, 2 \mathrm{H}), 4.35(\mathrm{dd}, J=11.0$, $7.0 \mathrm{~Hz}, 1 \mathrm{H}), 3.94(\mathrm{~s}, 3 \mathrm{H}), 2.73(\mathrm{~d}, J=11.0 \mathrm{~Hz}, 1 \mathrm{H}), 2.72$ (d, J = 7.0 Hz, 1H), 2.46 (s, $3 \mathrm{H}) . \quad{ }^{13} \mathrm{C} \mathrm{NMR}\left(\mathrm{CDCl}_{3}\right) \delta 169.4,145.2,142.2,141.4,137.6,135.4,129.6,129.1$, $128.8,128.6,127.8,127.3,125.9,116.2,116.0,55.8,50.9,42.4,21.8$. Мp. 79.5-80.5 ${ }^{\circ} \mathrm{C} . \quad$ TLC: $\mathrm{R}_{\mathrm{f}} 0.36$ (hexane/EtOAc = 3:1). IR (KBr): 3007, 2362, 1713, 1596, 1276, 1363, 1174, 1085, 1029, 814, $709 \mathrm{~cm}^{-1}$. HRMS Calcd for $\mathrm{C}_{23} \mathrm{H}_{21} \mathrm{NO}_{4} \mathrm{~S}_{2} \mathrm{Na}:[\mathrm{M}+\mathrm{Na}]^{+}$, 462.0804. Found: $m / z$ 462.0783. HPLC (Daicel Chiralpak $\mathrm{IF}$, hexane $/ i-\mathrm{PrOH}=80 / 20$, flow rate $\left.=2.0 \mathrm{~mL} / \mathrm{min}, \lambda=254 \mathrm{~nm}, 30{ }^{\circ} \mathrm{C}\right): t_{\text {minor }}=11.3$ $\min , t_{\text {major }}=14.2 \mathrm{~min}$.

\section{7-Chloro-2-phenyl-5-tosyl-2,3-dihydrobenzo $[b][1,4]$ thiazepin-4(5H)-one $(3 \mathrm{~d})$.}<smiles>[3H]N1C(=O)CC(c2ccccc2)Sc2ccc(Cl)cc21</smiles>

Yield: $85 \%$ (56.6 mg), 89\% ee, white solid. $\quad[\alpha]_{\mathrm{D}}{ }^{18}-271.1\left(c 0.83, \mathrm{CH}_{2} \mathrm{Cl}_{2}\right) .{ }^{1} \mathrm{H} \mathrm{NMR}$ $\left(\mathrm{CDCl}_{3}\right) \delta 7.88(\mathrm{~d}, J=8.0 \mathrm{~Hz}, 2 \mathrm{H}), 7.80(\mathrm{~d}, J=2.0 \mathrm{~Hz}, 1 \mathrm{H}), 7.50(\mathrm{~d}, J=8.5 \mathrm{~Hz}, 1 \mathrm{H})$, $7.45(\mathrm{dd}, J=8.5,2.0 \mathrm{~Hz}, 1 \mathrm{H}), 7.33(\mathrm{~d}, J=8.0 \mathrm{~Hz}, 2 \mathrm{H}), 7.24-7.23(\mathrm{~m}, 3 \mathrm{H}), 6.90(\mathrm{~m}$, 2H), $4.42(\mathrm{dd}, J=10.5,7.5 \mathrm{~Hz}, 1 \mathrm{H}), 2.75(\mathrm{~d}, J=7.5 \mathrm{~Hz}, 1 \mathrm{H}), 2.75(\mathrm{~d}, J=10.5 \mathrm{~Hz}, 1 \mathrm{H})$, $2.47(\mathrm{~s}, 3 \mathrm{H}) .{ }^{13} \mathrm{C} \mathrm{NMR}\left(\mathrm{CDCl}_{3}\right) \delta 168.8,145.4,142.0,141.2,137.5,136.0,135.3$, 130.7 , 130.1, 129.6, 129.2, 128.9, 128.1, 126.0, 125.8, 50.6, 42.2, 21.8. Mp. 210.1-210.8 ${ }^{\circ} \mathrm{C} . \quad$ TLC: $\mathrm{R}_{\mathrm{f}} 0.37$ (hexane/EtOAc = 3:1). $\quad \mathrm{IR}(\mathrm{KBr}): 3084,2362,1711$, 1356, 1166, 1128, 767, 698, 677, $652 \mathrm{~cm}^{-1}$. HRMS Calcd for $\mathrm{C}_{22} \mathrm{H}_{18} \mathrm{ClNO}_{3} \mathrm{~S}_{2} \mathrm{Na}$ : $[\mathrm{M}+\mathrm{Na}]^{+}$, 466.0309. Found: $\mathrm{m} / z$ 466.0302. HPLC (Daicel Chiralpak IF, hexane $/ i-\mathrm{PrOH}=80 / 20$, flow rate $\left.=2.0 \mathrm{~mL} / \mathrm{min}, \lambda=254 \mathrm{~nm}, 30^{\circ} \mathrm{C}\right): t_{\text {minor }}=8.6 \mathrm{~min}$, $t_{\text {major }}=9.8 \mathrm{~min}$. 
7-Bromo-2-phenyl-5-tosyl-2,3-dihydrobenzo $[b][1,4]$ thiazepin-4(5H)-one $(3 \mathrm{e})$.<smiles>[3H]N1C(=O)CC(c2ccccc2)Sc2ccc(Br)cc21</smiles>

Yield: $87 \%$ (63.7 mg), 90\% ee, white solid. $\quad[\alpha]_{\mathrm{D}}{ }^{18}-223.0\left(c 0.74, \mathrm{CH}_{2} \mathrm{Cl}_{2}\right) . \quad{ }^{1} \mathrm{H} \mathrm{NMR}$ $\left(\mathrm{CDCl}_{3}\right) \delta 7.94(\mathrm{~d}, J=2.0 \mathrm{~Hz}, 1 \mathrm{H}), 7.88(\mathrm{~d}, J=8.5 \mathrm{~Hz}, 2 \mathrm{H}), 7.59(\mathrm{dd}, J=8.0,2.0 \mathrm{~Hz}$, $1 \mathrm{H}), 7.43(\mathrm{~d}, J=8.0 \mathrm{~Hz}, 1 \mathrm{H}), 7.33(\mathrm{~d}, J=8.5 \mathrm{~Hz}, 2 \mathrm{H}), 7.26-7.23(\mathrm{~m}, 3 \mathrm{H}), 6.90(\mathrm{~d}, J=$ $7.0 \mathrm{~Hz}, 1 \mathrm{H}), 6.89$ (d, $J=7.0 \mathrm{~Hz}, 1 \mathrm{H}), 4.44(\mathrm{dd}, J=10.0,8.0 \mathrm{~Hz}, 1 \mathrm{H}), 2.75$ (d, $J=8.0$ $\mathrm{Hz}, 1 \mathrm{H}), 2.74(\mathrm{~d}, J=10.0 \mathrm{~Hz}, 1 \mathrm{H}), 2.47(\mathrm{~s}, 3 \mathrm{H}) .{ }^{13} \mathrm{C} \mathrm{NMR}\left(\mathrm{CDCl}_{3}\right) \delta 168.8,145.4$, 142.0, 141.2, 137.7, 135.2, 133.5, 133.0, 129.6, 129.1, 18.9, 128.1, 126.6, 125.8, 123.8, 50.5, 42.2, 21.8. Mp. 195.0-196.0 ${ }^{\circ} \mathrm{C} . \quad$ TLC: $\mathrm{R}_{\mathrm{f}} 0.33$ (hexane/EtOAc = 3:1). IR (KBr): 3061, 2362, 1714, 1570, 1452, 1387, 1362, 1242, 1171, 1127, 1086, 814, 759, 707, $675 \mathrm{~cm}^{-1}$. HRMS Calcd for $\mathrm{C}_{22} \mathrm{H}_{18} \mathrm{BrNO}_{3} \mathrm{~S}_{2} \mathrm{Na}$ : $[\mathrm{M}+\mathrm{Na}]^{+}, 509.9804$. Found: $\mathrm{m} / \mathrm{z}$ 509.9795. HPLC (Daicel Chiralpak IF, hexane $/ i$-PrOH $=80 / 20$, flow rate $=2.0$ $\left.\mathrm{mL} / \mathrm{min}, \lambda=254 \mathrm{~nm}, 30^{\circ} \mathrm{C}\right): t_{\text {minor }}=8.9 \mathrm{~min}, t_{\text {major }}=10.3 \mathrm{~min}$.

\section{2-(4-Methylphenyl)-5-tosyl-2,3-dihydrobenzo[b][1,4]thiazepin-4(5H)-one (3f).}<smiles>Cc1ccc(C2CC(=O)N([13F])c3ccccc3S2)cc1</smiles>

Yield: $82 \%$ (52.0 mg), 98\% ee, white solid. $\quad[\alpha]_{\mathrm{D}}{ }^{18}-264.5\left(c 0.86, \mathrm{CH}_{2} \mathrm{Cl}_{2}\right) . \quad{ }^{1} \mathrm{H} \mathrm{NMR}$ $\left(\mathrm{CDCl}_{3}\right) \delta 7.91(\mathrm{~d}, J=8.5 \mathrm{~Hz}, 2 \mathrm{H}), 7.79(\mathrm{dd}, J=8.5,1.5 \mathrm{~Hz}, 1 \mathrm{H}), 7.63-7.59(\mathrm{~m}, 2 \mathrm{H})$, $7.47(\mathrm{dt}, J=8.5,1.5 \mathrm{~Hz}, 1 \mathrm{H}), 7.33(\mathrm{~d}, J=8.0 \mathrm{~Hz}, 2 \mathrm{H}), 7.04(\mathrm{~d}, J=8.5 \mathrm{~Hz}, 2 \mathrm{H}), 6.80(\mathrm{~d}$, $J=8.0 \mathrm{~Hz}, 2 \mathrm{H}), 4.46(\mathrm{dd}, J=12.0,6.5 \mathrm{~Hz}, 1 \mathrm{H}), 2.75(\mathrm{~d}, J=12.0 \mathrm{~Hz}, 1 \mathrm{H}), 2.73(\mathrm{~d}, J=$ $6.5 \mathrm{~Hz}, 1 \mathrm{H}), 2.37(\mathrm{~s}, 3 \mathrm{H}), 2.29(\mathrm{~s}, 3 \mathrm{H}) .{ }^{13} \mathrm{C} \mathrm{NMR}\left(\mathrm{CDCl}_{3}\right) \delta 169.2,145.1,140.2$, $139.5,137.7,136.9,135.5,130.5,130.1,129.9$, 129.6, 129.4, 129.0, 127.5, 125.7, 50.3, 42.4, 21.7, 21.0. Mp. 84.7-85.2 ${ }^{\circ} \mathrm{C}$. TLC: $\mathrm{R}_{\mathrm{f}} 0.34$ (hexane/EtOAc = 3:1). $\mathrm{IR}$ (KBr): 2967, 1712, 1596, 1465, 1437, 1363, 1170, 1086, 814, $669 \mathrm{~cm}^{-1}$. HRMS Calcd for $\mathrm{C}_{23} \mathrm{H}_{21} \mathrm{NO}_{3} \mathrm{~S}_{2} \mathrm{Na}$ : $[\mathrm{M}+\mathrm{Na}]^{+}$, 446.0855. Found: $\mathrm{m} / z$ 446.0844. HPLC (Daicel Chiralpak IF, hexane $/ i-\mathrm{PrOH}=80 / 20$, flow rate $\left.=2.0 \mathrm{~mL} / \mathrm{min}, \lambda=254 \mathrm{~nm}, 30^{\circ} \mathrm{C}\right): t_{\text {minor }}$ $=15.2 \mathrm{~min}, t_{\text {major }}=17.4 \mathrm{~min}$. 
2-(2-Methylphenyl)-5-tosyl-2,3-dihydrobenzo[b][1,4]thiazepin-4(5H)-one (3g).<smiles>Cc1ccccc1C1CC(=O)N([12F])c2ccccc2S1</smiles>

Yield: 76\% (48.6 mg), 94\% ee, white solid. $\quad[\alpha]_{\mathrm{D}}{ }^{18}-280.0\left(c 1.00, \mathrm{CH}_{2} \mathrm{Cl}_{2}\right) . \quad{ }^{1} \mathrm{H} \mathrm{NMR}$ $\left(\mathrm{CDCl}_{3}\right) \delta 7.90(\mathrm{~d}, J=8.0 \mathrm{~Hz}, 2 \mathrm{H}), 7.80(\mathrm{dd}, J=8.0,1.0 \mathrm{~Hz}, 1 \mathrm{H}), 7.61(\mathrm{dt}, J=7.5,2.0$ $\mathrm{Hz}, 1 \mathrm{H}), 7.49-7.42(\mathrm{~m}, 2 \mathrm{H}), 7.34(\mathrm{~d}, J=8.0 \mathrm{~Hz}, 2 \mathrm{H}), 7.13-7.09(\mathrm{~m}, 2 \mathrm{H}), 7.02(\mathrm{~m}, 1 \mathrm{H})$, $6.57(\mathrm{~d}, J=7.5 \mathrm{~Hz}, 1 \mathrm{H}), 4.70(\mathrm{dd}, J=12.5,6.0 \mathrm{~Hz}, 1 \mathrm{H}), 2.82(\mathrm{dd}, J=12.5,12.5 \mathrm{~Hz}$, $1 \mathrm{H}), 2.63(\mathrm{dd}, J=12.5,6.0 \mathrm{~Hz}, 1 \mathrm{H}), 2.47(\mathrm{~s}, 3 \mathrm{H}), 2.27(\mathrm{~s}, 3 \mathrm{H}) .{ }^{13} \mathrm{C} \mathrm{NMR}\left(\mathrm{CDCl}_{3}\right) \delta$ 169.5, 145.1, 140.4, 139.7, 137.2, 135.6, 134.4, 130.7, 130.4, 130.2, 129.8, 129.5, 129.1, 127.6, 127.2, 126.2, 124.5, 46.1, 41.0, 21.8, 19.5. Mp. 175.1-176.1 ${ }^{\circ} \mathrm{C} . \quad$ TLC: $\mathrm{R}_{\mathrm{f}}$ 0.33 (hexane/EtOAc = 3:1). IR $(\mathrm{KBr}): 2967,1709,1462,1437,1171,1088,760,668$ $\mathrm{cm}^{-1}$. HRMS Calcd for $\mathrm{C}_{23} \mathrm{H}_{21} \mathrm{NO}_{3} \mathrm{~S}_{2} \mathrm{Na}$ : $[\mathrm{M}+\mathrm{Na}]^{+}$, 446.0855. Found: $\mathrm{m} / z$ 446.0845. HPLC (Daicel Chiralpak IA, hexane $/ i-\mathrm{PrOH}=80 / 20$, flow rate $=2.0 \mathrm{~mL} / \mathrm{min}, \lambda=254$ $\left.\mathrm{nm}, 30^{\circ} \mathrm{C}\right): t_{\text {minor }}=6.2 \mathrm{~min}, t_{\text {major }}=7.8 \mathrm{~min}$.

2-(4-Methoxyphenyl)-5-tosyl-2,3-dihydrobenzo[b][1,4]thiazepin-4(5H)-one (3h).<smiles>COc1ccc([C@@H]2CC(=O)N([13F])c3ccccc3S2)cc1</smiles>

Yield: $78 \%$ (51.4 mg), 97\% ee, white solid. $\quad[\alpha]_{\mathrm{D}}{ }^{18}-213.0\left(c 0.81, \mathrm{CH}_{2} \mathrm{Cl}_{2}\right) . \quad{ }^{1} \mathrm{H} \mathrm{NMR}$ $\left(\mathrm{CDCl}_{3}\right) \delta 7.88(\mathrm{~d}, J=8.0 \mathrm{~Hz}, 2 \mathrm{H}), 7.77(\mathrm{dd}, J=8.0,1.5 \mathrm{~Hz}, 1 \mathrm{H}), 7.62-7.57(\mathrm{~m}, 2 \mathrm{H})$, $7.46(\mathrm{dt}, J=7.5,1.5 \mathrm{~Hz}, 1 \mathrm{H}), 7.31(\mathrm{~d}, J=8.0 \mathrm{~Hz}, 2 \mathrm{H}), 6.82(\mathrm{~m}, 2 \mathrm{H}), 6.75(\mathrm{~m}, 2 \mathrm{H}), 4.40$ $(\mathrm{dd}, J=11.0,7.0 \mathrm{~Hz}, 1 \mathrm{H}), 3.75(\mathrm{~s}, 3 \mathrm{H}), 2.71(\mathrm{~d}, J=11.0 \mathrm{~Hz}, 1 \mathrm{H}), 2.71(\mathrm{~d}, J=7.0 \mathrm{~Hz}$, $1 \mathrm{H}), 2.46(\mathrm{~s}, 3 \mathrm{H}) . \quad{ }^{13} \mathrm{C} \mathrm{NMR}\left(\mathrm{CDCl}_{3}\right) \delta 169.2,159.1,145.1,140.2,136.9,135.5,134.7$, $130.5,130.1,129.9,129.6,129.0,127.5,127.0,114.0,55.2,50.2,42.6,21.8$. Мp. 79.5-80.5 ${ }^{\circ} \mathrm{C} . \quad$ TLC: $\mathrm{R}_{\mathrm{f}} 0.23$ (hexane/EtOAc = 3:1). IR (KBr): 2960, 1713, 1596, 1512, 1363, 1254, 1170, 1030, $668 \mathrm{~cm}^{-1}$. HRMS Calcd for $\mathrm{C}_{23} \mathrm{H}_{22} \mathrm{NO}_{4} \mathrm{~S}_{2}:[\mathrm{M}+\mathrm{H}]^{+}$, 440.0985. Found: $m / z$ 4400975. HPLC (Daicel Chiralpak IA, hexane $i-\mathrm{PrOH}=80 / 20$, flow rate $\left.=2.0 \mathrm{~mL} / \mathrm{min}, \lambda=254 \mathrm{~nm}, 30^{\circ} \mathrm{C}\right): t_{\text {minor }}=10.4 \mathrm{~min}, t_{\text {major }}=16.2 \mathrm{~min}$. 

(3i).<smiles>O=C1C[C@H](c2ccc(C(F)(F)F)cc2)Sc2ccccc2N1[As]</smiles>

Yield: $86 \%$ (61.7 mg), 98\% ee, white solid. $\quad[\alpha]_{\mathrm{D}}{ }^{18}-238.5\left(c 0.87, \mathrm{CH}_{2} \mathrm{Cl}_{2}\right) . \quad{ }^{1} \mathrm{H} \mathrm{NMR}$ $\left(\mathrm{CDCl}_{3}\right) \delta 7.88(\mathrm{~d}, J=8.0 \mathrm{~Hz}, 2 \mathrm{H}), 7.80(\mathrm{dd}, J=8.0,1.0 \mathrm{~Hz}, 1 \mathrm{H}), 7.63(\mathrm{dt}, J=8.0,1.5$ $\mathrm{Hz}, 1 \mathrm{H}), 7.57(\mathrm{dd}, J=7.5,1.5 \mathrm{~Hz}, 1 \mathrm{H}), 7.50-7.47(\mathrm{~m}, 3 \mathrm{H}), 7.32(\mathrm{~d}, J=8.0 \mathrm{~Hz}, 2 \mathrm{H})$, $7.03(\mathrm{~d}, J=7.5 \mathrm{~Hz}, 2 \mathrm{H}), 4.46(\mathrm{dd}, J=9.5,8.0 \mathrm{~Hz}, 1 \mathrm{H}), 2.73(\mathrm{~d}, J=8.0 \mathrm{~Hz}, 1 \mathrm{H}), 2.73(\mathrm{~d}$, $J=9.5 \mathrm{~Hz}, 1 \mathrm{H}), 2.46(\mathrm{~s}, 3 \mathrm{H}) . \quad{ }^{13} \mathrm{C} \mathrm{NMR}\left(\mathrm{CDCl}_{3}\right) \delta 168.7,146.1,145.3,140.3,136.8$, $135.8,135.4,130.7,130.6,130.4,130.1$ (q, $J=32.1 \mathrm{~Hz}$ ), 129.6, 129.1, 126.3, 125.8 (q, $J=3.4 \mathrm{~Hz}), 123.7(\mathrm{q}, J=272.1 \mathrm{~Hz}), 49.8,42.0,21.8 .{ }^{19} \mathrm{~F} \mathrm{NMR}\left(\mathrm{CDCl}_{3}\right) \delta 99.1 . \quad$ Mp. 81.8-82.8 ${ }^{\circ} \mathrm{C} . \quad$ TLC: $\mathrm{R}_{\mathrm{f}} 0.33$ (hexane/EtOAc = 3:1). IR (KBr): 2966, 1716, 1405, 1439, 1358, 1327, 1167, 1103, 1068, 834, $678 \mathrm{~cm}^{-1}$. HRMS Calcd for $\mathrm{C}_{23} \mathrm{H}_{18} \mathrm{~F}_{3} \mathrm{NO}_{3} \mathrm{~S}_{2} \mathrm{Na}:[\mathrm{M}+\mathrm{Na}]^{+}$, 500.0572. Found: $\mathrm{m} / z$ 500.0558. HPLC (Daicel Chiralpak IA, hexane $/ i-\mathrm{PrOH}=80 / 20$, flow rate $=2.0 \mathrm{~mL} / \mathrm{min}, \lambda=254 \mathrm{~nm}, 30{ }^{\circ} \mathrm{C}$ ): $t_{\text {minor }}=7.9 \mathrm{~min}, t_{\text {major }}=14.6 \mathrm{~min}$.

2-(4-Bromophenyl)-5-tosyl-2,3-dihydrobenzo[b][1,4] thiazepin-4(5H)-one (3j).<smiles>O=C1CC(c2ccc(Br)cc2)Sc2ccccc2N1[As]</smiles>

Yield: 99\% (77.5 mg), 97\% ee, white solid. $\quad[\alpha]_{\mathrm{D}}{ }^{18}-236.8\left(c 0.76, \mathrm{CH}_{2} \mathrm{Cl}_{2}\right) . \quad{ }^{1} \mathrm{H} \mathrm{NMR}$ $\left(\mathrm{CDCl}_{3}\right) \delta 7.88(\mathrm{~d}, J=8.0 \mathrm{~Hz}, 2 \mathrm{H}), 7.78(\mathrm{dd}, J=7.5,1.5 \mathrm{~Hz}, 1 \mathrm{H}), 7.62(\mathrm{dt}, J=7.5,1.5$ $\mathrm{Hz}, 1 \mathrm{H}), 7.56(\mathrm{dd}, J=7.5,1.5 \mathrm{~Hz}, 1 \mathrm{H}), 7.47$ (dt, $J=7.5,1.5 \mathrm{~Hz}, 1 \mathrm{H}), 7.35$ (d, $J=8.0$ $\mathrm{Hz}, 2 \mathrm{H}), 7.32(\mathrm{~d}, J=8.0 \mathrm{~Hz}, 2 \mathrm{H}), 6.77(\mathrm{~d}, J=8.0 \mathrm{~Hz}, 2 \mathrm{H}), 4.38(\mathrm{dd}, J=11.0,7.0 \mathrm{~Hz}$, $1 \mathrm{H}), 2.70(\mathrm{~d}, J=7.0 \mathrm{~Hz}, 1 \mathrm{H}), 2.69(\mathrm{~d}, J=11.0 \mathrm{~Hz}, 1 \mathrm{H}), 2.46(\mathrm{~s}, 3 \mathrm{H}) .{ }^{13} \mathrm{C} \mathrm{NMR}$ $\left(\mathrm{CDCl}_{3}\right) \delta 168.8,145.2,141.3,140.2,136.8,131.9,130.6,130.43,130.39,130.0,129.6$, 129.1, 127.6, 127.0, 121.7, 49.8, 42.1, 21.8. Mp. 88.7-89.7 ${ }^{\circ} \mathrm{C}$. TLC: $\mathrm{R}_{\mathrm{f}} 0.31$ (hexane/EtOAc = 3:1). IR (KBr): 2967, 1715, 1488, 1465, 1437, 1356, 1164, 1088, 
1009, 824, $677 \mathrm{~cm}^{-1}$. HRMS Calcd for $\mathrm{C}_{22} \mathrm{H}_{18} \mathrm{BrNO}_{3} \mathrm{~S}_{2} \mathrm{Na}:[\mathrm{M}+\mathrm{Na}]^{+}, 509.9804$. Found: $m / z$ 509.9794. HPLC (Daicel Chiralpak IA, hexane $/ i-\mathrm{PrOH}=80 / 20$, flow rate $\left.=2.0 \mathrm{~mL} / \mathrm{min}, \lambda=254 \mathrm{~nm}, 30{ }^{\circ} \mathrm{C}\right): t_{\text {minor }}=9.6 \mathrm{~min}, t_{\text {major }}=16.3 \mathrm{~min}$.

\section{2-(Furan-2-yl)-5-tosyl-2,3-dihydrobenzo[b][1,4]thiazepin-4(5H)-one (3k).}<smiles>O=C1C[C@H](c2ccco2)Sc2ccccc2N1[As]</smiles>

Yield: 78\% (46.9 mg), 97\% ee, white solid. $\quad[\alpha]_{\mathrm{D}}{ }^{18}-331.2\left(c 0.77, \mathrm{CH}_{2} \mathrm{Cl}_{2}\right) . \quad{ }^{1} \mathrm{H} \mathrm{NMR}$ $\left(\mathrm{CDCl}_{3}\right) \delta 7.86(\mathrm{~d}, J=8.0 \mathrm{~Hz}, 2 \mathrm{H}), 7.75(\mathrm{dd}, J=8.0,1.5 \mathrm{~Hz}, 1 \mathrm{H}), 7.60-7.56(\mathrm{~m}, 1 \mathrm{H})$, $7.49(\mathrm{dd}, J=7.5,1.5 \mathrm{~Hz}, 1 \mathrm{H}), 7.41(\mathrm{dt}, J=7.5,1.0 \mathrm{~Hz}, 1 \mathrm{H}), 7.31(\mathrm{~d}, J=8.0 \mathrm{~Hz}, 2 \mathrm{H})$, $7.25(\mathrm{dd}, J=2.0,1.0 \mathrm{~Hz}, 1 \mathrm{H}), 6.21(\mathrm{dd}, J=3.5,2.0 \mathrm{~Hz}, 1 \mathrm{H}), 5.83(\mathrm{~d}, J=3.5 \mathrm{~Hz}, 1 \mathrm{H})$, $4.49(\mathrm{dd}, J=12.0,6.0 \mathrm{~Hz}, 1 \mathrm{H}), 2.78(\mathrm{dd}, J=8.0,6.0 \mathrm{~Hz}, 1 \mathrm{H}), 2.65(\mathrm{t}, J=12.0 \mathrm{~Hz}, 1 \mathrm{H})$, $2.45(\mathrm{~s}, 3 \mathrm{H}) .{ }^{13} \mathrm{C} \mathrm{NMR}\left(\mathrm{CDCl}_{3}\right) \delta 168.8,153.3,145.2,142.4,140.2,137.3,135.3$, 130.4, 130.3, 129.8, 129.6, 129.1, 127.2, 110.3, 105.8, 43.8, 39.7, 21.8. Мp. decomposition. $\quad$ TLC: $\mathrm{R}_{\mathrm{f}} 0.26$ (hexane/EtOAc $=3: 1$ ). $\quad$ IR $(\mathrm{KBr}): 2967,1714,1596$, 1465, 1439, 1363, 1289, 1170, 1086, 1010, 905, 732, $668 \mathrm{~cm}^{-1}$. HRMS Calcd for $\mathrm{C}_{20} \mathrm{H}_{17} \mathrm{NO}_{4} \mathrm{~S}_{2} \mathrm{Na}:[\mathrm{M}+\mathrm{Na}]^{+}$, 422.0991. Found: $m / z$ 422.0980. HPLC (Daicel Chiralpak $\mathrm{IF}$, hexane $/ i-\mathrm{PrOH}=80 / 20$, flow rate $\left.=2.0 \mathrm{~mL} / \mathrm{min}, \lambda=254 \mathrm{~nm}, 30{ }^{\circ} \mathrm{C}\right): t_{\text {minor }}=12.0$ $\min , t_{\text {major }}=16.9 \mathrm{~min}$.

\section{2-Ethyl-5-tosyl-2,3-dihydrobenzo[b][1,4]thiazepin-4(5H)-one $(3 \mathrm{l})$.}<smiles>[B]N1C(=O)CC(CC)Sc2ccccc21</smiles>

Yield: $96 \%$ (51.8 mg), 93\% ee, white solid. $\quad[\alpha]_{\mathrm{D}}{ }^{18}-142.4\left(c 0.79, \mathrm{CH}_{2} \mathrm{Cl}_{2}\right) . \quad{ }^{1} \mathrm{H} \mathrm{NMR}$ $\left(\mathrm{CDCl}_{3}\right) \delta 7.84(\mathrm{~d}, J=7.5 \mathrm{~Hz}, 2 \mathrm{H}), 7.69(\mathrm{~d}, J=7.5 \mathrm{~Hz}, 1 \mathrm{H}), 7.60(\mathrm{dd}, J=7.5,1.0 \mathrm{~Hz}$, $1 \mathrm{H}), 7.53(\mathrm{dt}, J=8.0,1.5 \mathrm{~Hz}, 1 \mathrm{H}), 7.40(\mathrm{dt}, J=7.5,1.5 \mathrm{~Hz}, 1 \mathrm{H}), 7.28(\mathrm{~d}, J=7.5 \mathrm{~Hz}$, 2H), $3.14(\mathrm{~m}, 1 \mathrm{H}), 2.55(\mathrm{dd}, J=12.0,6.0 \mathrm{~Hz}, 1 \mathrm{H}), 2.43(\mathrm{~s}, 3 \mathrm{H}), 2.06(\mathrm{dd}, J=12.0,12.0$ $\mathrm{Hz}, 1 \mathrm{H}), 1.34(\mathrm{~m}, 2 \mathrm{H}), 0.87(\mathrm{t}, J=7.0 \mathrm{~Hz}, 3 \mathrm{H}) .{ }^{13} \mathrm{C} \mathrm{NMR}\left(\mathrm{CDCl}_{3}\right) \delta 170.0,144.9$, 140.5, 137.1, 135.5, 130.3, 129.9, 129.6, 129.5, 129.0, 127.2, 49.9, 41.6, 29.7, 21.7, 11.3. Mp. $36.8-37.8{ }^{\circ} \mathrm{C}$. TLC: $\mathrm{R}_{\mathrm{f}} 0.40$ (hexane/EtOAc = 3:1). $\quad$ IR $(\mathrm{KBr}): 2955$, 
1718, 1598, 1467, 1440, 1366, 1166, 1141, 1085, 893, 761, $676 \mathrm{~cm}^{-1}$. HRMS Calcd for $\mathrm{C}_{18} \mathrm{H}_{20} \mathrm{NO}_{3} \mathrm{~S}_{2}$ : $[\mathrm{M}+\mathrm{H}]^{+}, 362.0879$. Found: $m / z$ 362.0868. HPLC (Daicel Chiralpak $\mathrm{IF}$, hexane $/ i-\mathrm{PrOH}=80 / 20$, flow rate $\left.=2.0 \mathrm{~mL} / \mathrm{min}, \lambda=254 \mathrm{~nm}, 30^{\circ} \mathrm{C}\right): t_{\text {minor }}=11.2$ $\min , t_{\text {major }}=15.3 \mathrm{~min}$.

\section{2-Isopropyl-5-tosyl-2,3-dihydrobenzo[b][1,4]thiazepin-4(5H)-one (3m).}<smiles>CC(C)C1CC(=O)N([12S])c2ccccc2S1</smiles>

Yield: 99\% (57.8 mg), 97\% ee, white solid. $\quad[\alpha]_{\mathrm{D}}{ }^{18}-171.1\left(c 0.95, \mathrm{CH}_{2} \mathrm{Cl}_{2}\right) . \quad{ }^{1} \mathrm{H} \mathrm{NMR}$ $\left(\mathrm{CDCl}_{3}\right) \delta 7.84(\mathrm{~d}, J=8.5 \mathrm{~Hz}, 2 \mathrm{H}), 7.68(\mathrm{dd}, J=8.0,1.0 \mathrm{~Hz}, 1 \mathrm{H}), 7.64(\mathrm{dd}, J=7.5,1.5$ $\mathrm{Hz}, 1 \mathrm{H}), 7.51$ (dt, $J=8.0,1.5 \mathrm{~Hz}, 1 \mathrm{H}), 7.39$ (dt, $J=7.5,1.0 \mathrm{~Hz}, 1 \mathrm{H}), 7.28$ (d, $J=8.5 \mathrm{~Hz}$, 2H), $3.06(\mathrm{~m}, 1 \mathrm{H}), 2.53(\mathrm{dd}, J=12.5,5.0 \mathrm{~Hz}, 1 \mathrm{H}), 2.43(\mathrm{~s}, 3 \mathrm{H}), 2.23(\mathrm{dd}, J=12.5,12.5$ $\mathrm{Hz}, 1 \mathrm{H}), 1.57(\mathrm{~m}, 1 \mathrm{H}), 0.86(\mathrm{~d}, J=6.5 \mathrm{~Hz}, 3 \mathrm{H}), 0.78(\mathrm{~d}, J=7.0 \mathrm{~Hz}, 3 \mathrm{H}) .{ }^{13} \mathrm{C} \mathrm{NMR}$ $\left(\mathrm{CDCl}_{3}\right) \delta 170.4,144.9,140.3,136.9,135.5,130.1,129.65,129.59,129.54,129.0$, 128.1, 54.8, 39.5, 21.7, 19.5, 19.3. Mp. 106.8-107.8 ${ }^{\circ} \mathrm{C} . \quad$ TLC: $\mathrm{R}_{\mathrm{f}} 0.31$ (hexane/EtOAc = 3:1). IR $(\mathrm{KBr}):$ 2962, 1714, 1596, 1465, 1438, 1363, 1171, 1087, 903, $668 \mathrm{~cm}^{-1}$. HRMS Calcd for $\mathrm{C}_{19} \mathrm{H}_{22} \mathrm{NO}_{3} \mathrm{~S}_{2}:[\mathrm{M}+\mathrm{H}]^{+}$, 376.1036. Found: $\mathrm{m} / z$ 376.1024. HPLC (Daicel Chiralpak IF, hexane $/ i$-PrOH $=80 / 20$, flow rate $=2.0$ $\left.\mathrm{mL} / \mathrm{min}, \lambda=254 \mathrm{~nm}, 30{ }^{\circ} \mathrm{C}\right): t_{\text {minor }}=9.8 \mathrm{~min}, t_{\text {major }}=15.7 \mathrm{~min}$.

\section{7-Chloro-2-(p-tolyl)-5-tosyl-2,3-dihydrobenzo[b][1,4]thiazepin-4(5H)-one (3n).}<smiles>Cc1ccc(C2CC(=O)N([13F])c3cc(Cl)ccc3S2)cc1</smiles>

Yield: 74\% (50.7 mg), 92\% ee, white solid. $[\alpha]_{\mathrm{D}}{ }^{18}-262.5\left(c 1.00, \mathrm{CH}_{2} \mathrm{Cl}_{2}\right) . \quad{ }^{1} \mathrm{H} \mathrm{NMR}$ $\left(\mathrm{CDCl}_{3}\right) \delta 7.88(\mathrm{~d}, J=8.5 \mathrm{~Hz}, 2 \mathrm{H}), 7.79(\mathrm{~d}, J=2.0 \mathrm{~Hz}, 1 \mathrm{H}), 7.49(\mathrm{~d}, J=8.0 \mathrm{~Hz}, 1 \mathrm{H})$, $7.44(\mathrm{dd}, J=8.0,2.0 \mathrm{~Hz}, 1 \mathrm{H}), 7.33(\mathrm{~d}, J=8.5 \mathrm{~Hz}, 2 \mathrm{H}), 7.04(\mathrm{~d}, J=8.0 \mathrm{~Hz}, 2 \mathrm{H}), 6.78(\mathrm{~d}$, $J=8.0 \mathrm{~Hz}, 2 \mathrm{H}), 4.40(\mathrm{t}, J=8.5 \mathrm{~Hz}, 1 \mathrm{H}), 2.73(\mathrm{~d}, J=8.5 \mathrm{~Hz}, 2 \mathrm{H}), 2.46(\mathrm{~s}, 3 \mathrm{H}), 2.29$ (s, $3 \mathrm{H}) . \quad{ }^{13} \mathrm{C} \mathrm{NMR}\left(\mathrm{CDCl}_{3}\right) \delta 168.8,145.4,141.1,139.1,138.0,137.5,135.9,135.2$, $130.6,130.0,129.6,129.5,129.1,126.1,125.7,50.5,42.2,21.8,21.0 . \quad$ Мp. 
88.8-89.8 ${ }^{\circ} \mathrm{C} . \quad$ TLC: $\mathrm{R}_{\mathrm{f}} 0.40$ (hexane/EtOAc = 3:1). $\quad$ IR (KBr): 2968, 2921, 1717, 1576, 1457, 1394, 1363, 1260, 1171, 1127, 1086, 1000, 815, 677, $655 \mathrm{~cm}^{-1}$. HRMS Calcd for $\mathrm{C}_{23} \mathrm{H}_{20} \mathrm{ClNO}_{3} \mathrm{~S}_{2} \mathrm{Na}:[\mathrm{M}+\mathrm{Na}]^{+}$, 480.0465. Found: $m / z$ 480.0455. HPLC (Daicel Chiralpak IF, hexane $/ i-\mathrm{PrOH}=80 / 20$, flow rate $=2.0 \mathrm{~mL} / \mathrm{min}, \lambda=254 \mathrm{~nm}$, $\left.30{ }^{\circ} \mathrm{C}\right): t_{\text {minor }}=8.5 \mathrm{~min}, t_{\text {major }}=9.8 \mathrm{~min}$.

\section{7-Chloro-2-(4-methoxyphenyl)-5-tosyl-2,3-dihydrobenzo[b][1,4] thiazepin-4(5H)-on} e (3o).<smiles>COc1ccc([C@@H]2CC(=O)N([13F])c3cc(Cl)ccc3S2)cc1</smiles>

Yield: $73 \%$ (52.1 mg), 91\% ee, white solid. $\quad[\alpha]_{\mathrm{D}}{ }^{18}-254.7\left(c 1.06, \mathrm{CH}_{2} \mathrm{Cl}_{2}\right) . \quad{ }^{1} \mathrm{H} \mathrm{NMR}$ $\left(\mathrm{CDCl}_{3}\right) \delta 8.87(\mathrm{~d}, J=8.0 \mathrm{~Hz}, 2 \mathrm{H}), 7.79(\mathrm{~d}, J=2.0 \mathrm{~Hz}, 1 \mathrm{H}), 7.48(\mathrm{~d}, J=8.0 \mathrm{~Hz}, 1 \mathrm{H})$, $7.44(\mathrm{dd}, J=8.0,2.0 \mathrm{~Hz}, 1 \mathrm{H}), 7.32(\mathrm{~d}, J=8.0 \mathrm{~Hz}, 2 \mathrm{H}), 6.82(\mathrm{~d}, J=8.5 \mathrm{~Hz}, 2 \mathrm{H}), 6.75(\mathrm{~d}$, $J=8.5 \mathrm{~Hz}, 2 \mathrm{H}), 4.40(\mathrm{dd}, J=10.5,7.5 \mathrm{~Hz}, 1 \mathrm{H}), 3.75(\mathrm{~s}, 3 \mathrm{H}), 2.72(\mathrm{~d}, J=10.5 \mathrm{~Hz}, 1 \mathrm{H})$, $2.72(\mathrm{~d}, J=7.5 \mathrm{~Hz}, 1 \mathrm{H}), 2.46(\mathrm{~s}, 3 \mathrm{H}) . \quad{ }^{13} \mathrm{C} \mathrm{NMR}\left(\mathrm{CDCl}_{3}\right) \delta 168.8,159.2,145.4,141.1$, 137.5, 135.9, 135.2, 134.2, 130.6, 130.0, 129.6, 129.1, 127.0, 126.1, 114.1, 55.2, 50.3, 42.3, 21.8. Mp. 80.0-80.5 ${ }^{\circ} \mathrm{C}$. TLC: $\mathrm{R}_{\mathrm{f}} 0.26$ (hexane/EtOAc = 3:1). IR (KBr): 2967, 1717, 1576, 1512, 1457, 1394, 1362, 1252, 1171, 1128, 1032, 830, 814, 677, 656 $\mathrm{cm}^{-1}$. HRMS Calcd for $\mathrm{C}_{23} \mathrm{H}_{20} \mathrm{ClNO}_{4} \mathrm{~S}_{2} \mathrm{Na}:[\mathrm{M}+\mathrm{Na}]^{+}, 496.0414$. Found: $\mathrm{m} / z$ 496.0404. HPLC (Daicel Chiralpak IF, hexane $/ i-\mathrm{PrOH}=80 / 20$, flow rate $=2.0 \mathrm{~mL} / \mathrm{min}, \lambda=254$ $\left.\mathrm{nm}, 30^{\circ} \mathrm{C}\right): t_{\text {minor }}=12.3 \mathrm{~min}, t_{\text {major }}=13.7 \mathrm{~min}$.

\section{2-(4-Bromophenyl)-7-methyl-5-tosyl-2,3-dihydrobenzo[b][1,4]thiazepin-4(5H)-one} (3p).<smiles>Cc1ccc2c(c1)N([13CH3])C(=O)C[C@@H](c1ccc(Br)cc1)S2</smiles>

Yield: 95\% (71.5 mg), 97\% ee, white solid. $\quad[\alpha]_{\mathrm{D}}{ }^{18}-302.6\left(c 0.95, \mathrm{CH}_{2} \mathrm{Cl}_{2}\right) . \quad{ }^{1} \mathrm{H} \mathrm{NMR}$ $\left(\mathrm{CDCl}_{3}\right) \delta 7.87(\mathrm{~d}, J=8.0 \mathrm{~Hz}, 2 \mathrm{H}), 7.59(\mathrm{~m}, 1 \mathrm{H}), 7.42(\mathrm{~d}, J=7.5 \mathrm{~Hz}, 1 \mathrm{H}), 7.35(\mathrm{~m}, 2 \mathrm{H})$, 
$7.31(\mathrm{~d}, J=8.0 \mathrm{~Hz}, 1 \mathrm{H}), 7.26(\mathrm{~m}, 2 \mathrm{H}), 7.18(\mathrm{~m}, 2 \mathrm{H}), 4.33(\mathrm{dd}, J=10.0,9.0 \mathrm{~Hz}, 1 \mathrm{H})$,

$2.68(\mathrm{~d}, J=10.0 \mathrm{~Hz}, 1 \mathrm{H}), 2.68(\mathrm{~d}, J=9.0 \mathrm{~Hz}, 1 \mathrm{H}), 2.51(\mathrm{~s}, 3 \mathrm{H}), 2.45(\mathrm{~s}, 3 \mathrm{H}) .{ }^{13} \mathrm{C}$ NMR $\left(\mathrm{CDCl}_{3}\right) \delta 169.0,145.1,141.4,141.2,140.0,136.5,135.4,131.8,131.2,130.9$, 129.6, 129.0, 127.6, 123.4, 121.6, 49.8, 42.1, 21.7, 21.3. Mp. 93.2-94.2 ${ }^{\circ} \mathrm{C} . \quad$ TLC: $\mathrm{R}_{\mathrm{f}}$ 0.40 (hexane/EtOAc $=3: 1) . \quad$ IR $(\mathrm{KBr}): 2921,1715,1599,1487,1359,1254,1166$, 1088, 1008, 823, 812, 688, $656 \mathrm{~cm}^{-1}$. HRMS Calcd for $\mathrm{C}_{23} \mathrm{H}_{20} \mathrm{BrNO}_{3} \mathrm{~S}_{2} \mathrm{Na}$ : $[\mathrm{M}+\mathrm{Na}]^{+}$, 523.9960. Found: $m / z$ 523.9949. HPLC (Daicel Chiralpak IF, hexane $/ i-\mathrm{PrOH}=80 / 20$, flow rate $\left.=2.0 \mathrm{~mL} / \mathrm{min}, \lambda=254 \mathrm{~nm}, 30^{\circ} \mathrm{C}\right): t_{\text {minor }}=10.5 \mathrm{~min}, t_{\text {major }}=12.4 \mathrm{~min}$.

\section{2-Ethyl-7-methoxy-5-tosyl-2,3-dihydrobenzo[b][1,4]thiazepin-4(5H)-one (3q).}<smiles>CCC1CC(=O)N([12S])c2cc(OC)ccc2S1</smiles>

Yield: 94\% (55.0 mg), 93\% ee, white solid. $\quad[\alpha]_{\mathrm{D}}{ }^{18}-136.2\left(c 1.12, \mathrm{CH}_{2} \mathrm{Cl}_{2}\right) . \quad{ }^{1} \mathrm{H} \mathrm{NMR}$ $\left(\mathrm{CDCl}_{3}\right) \delta 7.83(\mathrm{~d}, J=8.0 \mathrm{~Hz}, 2 \mathrm{H}), 7.43(\mathrm{~d}, J=7.5 \mathrm{~Hz}, 1 \mathrm{H}), 7.28(\mathrm{~d}, J=8.0 \mathrm{~Hz}, 2 \mathrm{H})$, $7.22(\mathrm{~d}, J=2.5 \mathrm{~Hz}, 1 \mathrm{H}), 6.93(\mathrm{dd}, J=7.5,2.5 \mathrm{~Hz}, 1 \mathrm{H}), 3.89(\mathrm{~s}, 3 \mathrm{H}), 3.06(\mathrm{~m}, 1 \mathrm{H}), 2.53$ (dd, $J=12.5,6.0 \mathrm{~Hz}, 1 \mathrm{H}), 2.43(\mathrm{~s}, 3 \mathrm{H}), 2.03(\mathrm{dd}, J=7.0,6.0 \mathrm{~Hz}, 1 \mathrm{H}), 1.31(\mathrm{~m}, 2 \mathrm{H})$ $0.85(\mathrm{t}, J=7.5 \mathrm{~Hz}, 3 \mathrm{H}) . \quad{ }^{13} \mathrm{C} \mathrm{NMR}\left(\mathrm{CDCl}_{3}\right) \delta 170.2,160.6,144.9,141.6,137.6,135.5$, 129.6, 128.9, 118.0, 115.80, 115.76, 55.8, 50.2, 41.7, 29.4, 21.7, 11.3. Мp. 143.6-144.6 ${ }^{\circ} \mathrm{C} . \quad$ TLC: $\mathrm{R}_{\mathrm{f}} 0.29$ (hexane/EtOAc = 3:1). IR (KBr): 2966, 2934, 1714, 1596, 1472, 1362, 1244, 1173, 1085, 1028, 895, 813, 711, 685, $654 \mathrm{~cm}^{-1}$. HRMS Calcd for $\mathrm{C}_{19} \mathrm{H}_{21} \mathrm{NO}_{4} \mathrm{~S}_{2} \mathrm{Na}$ : $[\mathrm{M}+\mathrm{Na}]^{+}$, 414.0804. Found: $m / z$ 414.0787. HPLC (Daicel Chiralpak IF, hexane $/ i-\mathrm{PrOH}=80 / 20$, flow rate $=2.0 \mathrm{~mL} / \mathrm{min}, \lambda=254 \mathrm{~nm}, 30^{\circ} \mathrm{C}$ ): $t_{\text {minor }}$ $=10.5 \mathrm{~min}, t_{\text {major }}=11.3 \mathrm{~min}$.

\section{7-Bromo-2-(furan-2-yl)-5-tosyl-2,3-dihydrobenzo[b][1,4]thiazepin-4(5H)-one (3r).}<smiles>O=C1C[C@H](c2ccco2)Sc2ccc(Br)cc2N1[As]</smiles>

Yield: $70 \%$ (50.7 mg), 91\% ee, white solid. $[\alpha]_{\mathrm{D}}{ }^{18}-344.5\left(c 0.82, \mathrm{CH}_{2} \mathrm{Cl}_{2}\right) . \quad{ }^{1} \mathrm{H} \mathrm{NMR}$ $\left(\mathrm{CDCl}_{3}\right) \delta 7.91(\mathrm{~d}, J=2.0 \mathrm{~Hz}, 1 \mathrm{H}), 7.86(\mathrm{~d}, J=8.0 \mathrm{~Hz}, 2 \mathrm{H}), 7.54(\mathrm{dd}, J=8.0,2.0 \mathrm{~Hz}$, $1 \mathrm{H}), 7.34(\mathrm{~d}, J=8.0 \mathrm{~Hz}, 1 \mathrm{H}), 7.31(\mathrm{~d}, J=8.0 \mathrm{~Hz}, 2 \mathrm{H}), 7.25(\mathrm{~m}, 1 \mathrm{H}), 6.21(\mathrm{dd}, J=3.0$, 
$1.5 \mathrm{~Hz}, 1 \mathrm{H}), 5.86(\mathrm{~d}, J=3.0 \mathrm{~Hz}, 1 \mathrm{H}), 4.49(\mathrm{dd}, J=12.5,6.0 \mathrm{~Hz}, 1 \mathrm{H}), 2.80(\mathrm{dd}, J=12.5$, $6.0 \mathrm{~Hz}, 1 \mathrm{H}), 2.65(\mathrm{dd}, J=12.5,12.5 \mathrm{~Hz}, 1 \mathrm{H}), 2.46(\mathrm{~s}, 3 \mathrm{H}) .{ }^{13} \mathrm{C} \mathrm{NMR}\left(\mathrm{CDCl}_{3}\right) \delta 168.4$, 152.9, 145.4, 142.5, 141.1, 138.1, 135.1, 133.2, 133.0, 129.6, 129.1, 126.1, 123.9, 110.3, 106.0, 43.8, 39.6, 21.8. Mp. 93.2-94.2 ${ }^{\circ} \mathrm{C} . \quad$ TLC: $\mathrm{R}_{\mathrm{f}} 0.51$ (hexane/EtOAc = 3:1). IR (KBr): 1723, 1595, 1569, 1387, 1355, 1282, 1167, 1128, 1084, 1011, 992, 839, 740, 680, $655 \mathrm{~cm}^{-1}$. HRMS Calcd for $\mathrm{C}_{20} \mathrm{H}_{16} \mathrm{BrNO}_{4} \mathrm{~S}_{2} \mathrm{Na}$ : $[\mathrm{M}+\mathrm{Na}]^{+}$, 499.9596. Found: $\mathrm{m} / \mathrm{z}$ 499.9582. HPLC (Daicel Chiralpak IF, hexane $/ i$-PrOH $=80 / 20$, flow rate $=2.0$ $\left.\mathrm{mL} / \mathrm{min}, \lambda=254 \mathrm{~nm}, 30^{\circ} \mathrm{C}\right): t_{\text {minor }}=8.5 \mathrm{~min}, t_{\text {major }}=9.8 \mathrm{~min}$.

\section{(E)-S-(2-(4-Methylphenylsulfonamido)phenyl) 3-phenylprop-2-enethioate (4a).}<smiles>Nc1ccccc1SC(=O)/C=C/c1ccccc1</smiles>

White solid. ${ }^{1} \mathrm{H}$ NMR $\left(\mathrm{CDCl}_{3}\right) \delta 7.69(\mathrm{dd}, J=8.0,1.5 \mathrm{~Hz}, 1 \mathrm{H}), 7.64-7.61(\mathrm{~m}, 3 \mathrm{H})$, $7.57(\mathrm{~m}, 2 \mathrm{H}), 7.47-7.41(\mathrm{~m}, 4 \mathrm{H}), 7.34$ (dd, $J=7.5,1.5 \mathrm{~Hz}, 1 \mathrm{H}), 7.21-7.18(\mathrm{~m}, 3 \mathrm{H})$, $7.15(\mathrm{dt}, J=8.0,1.0 \mathrm{~Hz}, 1 \mathrm{H}), 6.69(\mathrm{~d}, J=16.0 \mathrm{~Hz}, 1 \mathrm{H}), 2.36(\mathrm{~s}, 3 \mathrm{H}) .{ }^{13} \mathrm{C} \mathrm{NMR}$ $\left(\mathrm{CDCl}_{3}\right) \delta 186.2,144.0,143.2,138.8,136.8,136.0,133.5,131.6,131.3,129.6,129.1$, 128.7, 127.3, 125.5, 123.0, 121.9, 118.6, 21.6. Mp. 139.2-140.2 ${ }^{\circ} \mathrm{C}$. TLC: $\mathrm{R}_{\mathrm{f}} 0.31$ (hexane/EtOAc = 3:1). IR (KBr): 3280, 1685, 1620, 1482, 1396, 1337, 1161, 1090, 1031, 911, 766, $667 \mathrm{~cm}^{-1}$. HRMS Calcd for $\mathrm{C}_{22} \mathrm{H}_{20} \mathrm{NO}_{3} \mathrm{~S}_{2}:[\mathrm{M}+\mathrm{H}]^{+}, 410.0879$. Found: $\mathrm{m} / \mathrm{z} 410.0877$.

\section{Procedure for synthesis of thiazesim ${ }^{8,9}$}

To a solution of $\mathrm{SmI}_{2}(10 \mathrm{~mL}, 0.10 \mathrm{M}, 1.0 \mathrm{mmol})$ in THF was added 3a $(69.6 \mathrm{mg}$, $0.17 \mathrm{mmol})$ followed by $\mathrm{H}_{2} \mathrm{O}(54 \mu \mathrm{L}, 3.0 \mathrm{mmol})$ and triethylamine $(0.28 \mathrm{~mL}, 2.0 \mathrm{mmol})$, and the mixture was stirred under argon atmosphere for $30 \mathrm{~min}$. The resulting mixture was diluted with $\mathrm{Et}_{2} \mathrm{O}(10 \mathrm{~mL})$ and washed with saturated aqueous $\mathrm{NaHCO}_{3}(10 \mathrm{~mL} \times$ 3). The organic layers were washed with brine, dried over $\mathrm{Na}_{2} \mathrm{SO}_{4}$, and concentrated in vacuo. Purification by flash silica gel column chromatography using hexane/EtOAc $(\mathrm{v} / \mathrm{v}=1 / 1)$ as an eluent gave the detosylated product 7 (27.6 mg, 64\% yield). 
<smiles>O=C1CC(c2ccccc2)Sc2ccccc2N1</smiles>

Yield: $64 \%$ yield $(27.6 \mathrm{mg}), 96 \%$ ee, white solid. $\quad[\alpha]_{\mathrm{D}}{ }^{18}-448.4\left(c 0.63, \mathrm{CHCl}_{3}\right) . \quad{ }^{1} \mathrm{H}$ NMR $\left(\mathrm{CDCl}_{3}\right) \delta 7.80$ (brs, $\left.1 \mathrm{H}\right), 7.67$ (dd, $\left.J=7.5,1.5 \mathrm{~Hz}, 1 \mathrm{H}\right), 7.43$ (ddd, $J=7.5,7.5$, $1.5 \mathrm{~Hz}, 1 \mathrm{H}), 7.34-7.23(\mathrm{~m}, 6 \mathrm{H}), 7.16(\mathrm{~d}, J=7.5 \mathrm{~Hz}, 1 \mathrm{H}), 4.88(\mathrm{dd}, J=11.5,5.5 \mathrm{~Hz}$, $1 \mathrm{H}), 2.89(\mathrm{dd}, J=12.5,11.5 \mathrm{~Hz}, 1 \mathrm{H}), 2.81(\mathrm{dd}, J=12.5,5.5 \mathrm{~Hz}, 1 \mathrm{H}) .{ }^{13} \mathrm{C} \mathrm{NMR}$ $\left(\mathrm{CDCl}_{3}\right) \delta 171.9,143.4,141.2,135.9,130.2,128.8,127.8,126.8,126.6,126.4,123.2$, 53.1, 41.4. TLC: $\mathrm{R}_{\mathrm{f}} 0.38$ (hexane/EtOAc $=1: 1$ ). HRMS Calcd for $\mathrm{C}_{15} \mathrm{H}_{14} \mathrm{NOS}$ : $[\mathrm{M}+\mathrm{H}]^{+}, 278.0610$. Found: $m / z$ 278.0606. HPLC (Daicel Chiralpak IF, hexane/i-PrOH $=80 / 20$, flow rate $=2.0 \mathrm{~mL} / \mathrm{min}, \lambda=254 \mathrm{~nm}, 30^{\circ} \mathrm{C}$ ): $t_{\text {major }}=5.9 \mathrm{~min}, t_{\text {minor }}=8.5 \mathrm{~min}$.

The absolute configuration of 7 was assigned as $(R)$ by comparing the optical rotation with the literature value. ${ }^{9}$

$[\alpha]_{\mathrm{D}}{ }^{18}-448.4\left(c 0.63, \mathrm{CHCl}_{3}\right)$ [lit. ${ }^{9}(R)$-2-phenyl-2,3-dihydrobenzo[ $\left.b\right][1,4]$ thiazepin$4(5 H)$-one $\left.(98 \% e e):[\alpha]_{\mathrm{D}}{ }^{25}-519.9\left(c 0.63, \mathrm{CHCl}_{3}\right)\right]$.

To a solution of $(R)$-2-phenyl-2,3-dihydrobenzo[b][1,4]thiazepin-4(5H)-one $\mathbf{7}$, 30.6 $\mathrm{mg}, \quad 0.12 \mathrm{mmol})$ in EtOAc $(1 \mathrm{~mL})$ was added 2-dimethylaminoethylchloride hydrochloride (34.6 mg, $0.24 \mathrm{mmol})$, followed by $\mathrm{K}_{2} \mathrm{CO}_{3}(66.3 \mathrm{mg}, 0.48 \mathrm{mmol})$ and $\mathrm{H}_{2} \mathrm{O}(10 \mu \mathrm{L})$. After the mixture was sttired for $12 \mathrm{~h}$ under reflux, it was cooled to ambient temperature. The organic layers were washed with $\mathrm{H}_{2} \mathrm{O}(5 \mathrm{~mL} \times 2)$ and brine. The combined organic layers were dried over $\mathrm{Na}_{2} \mathrm{SO}_{4}$, and concentrated in vacuo. Purification by flash silica gel column chromatography using $\mathrm{CHCl}_{3} / \mathrm{MeOH}$ $(\mathrm{v} / \mathrm{v}=10 / 1) \quad$ as $\quad$ an gave (R)-5-(2-(dimethylamino)ethyl)-2-phenyl-2,3-dihydrobenzo[b][1,4]thiazepin-4(5H)-one (8, $35.9 \mathrm{mg}, 0.11 \mathrm{mmol}, 92 \%$ yield). 
(R)-5-(2-(Dimethylamino)ethyl)-2-phenyl-2,3-dihydrobenzo[b][1,4]thiazepin-4(5H)one (8).<smiles>CNCCN1C(=O)CC(c2ccccc2)Sc2ccccc21</smiles>

Yield: $92 \%$ yield $(35.9 \mathrm{mg}), 97 \%$ ee, colorless oil. $\quad[\alpha]_{\mathrm{D}}{ }^{18}-416.7\left(c 0.33, \mathrm{CHCl}_{3}\right) \quad{ }^{1} \mathrm{H}$ $\operatorname{NMR}\left(\mathrm{CDCl}_{3}\right) \delta 7.62(\mathrm{~d}, J=7.5 \mathrm{~Hz}, 1 \mathrm{H}), 7.51-7.46(\mathrm{~m}, 2 \mathrm{H}), 7.27-7.14(\mathrm{~m}, 4 \mathrm{H})$, $7.14-7.10(\mathrm{~m}, 2 \mathrm{H}), 4.78(\mathrm{dd}, J=12.5,5.5 \mathrm{~Hz}, 1 \mathrm{H}), 4.33-4.26(\mathrm{~m}, 1 \mathrm{H}), 3.66(\mathrm{~m}, 1 \mathrm{H})$, 2.82-2.65 (m, 3H), $2.37(\mathrm{~m}, 1 \mathrm{H}), 2.24(\mathrm{~s}, 6 \mathrm{H}) .{ }^{13} \mathrm{C} \mathrm{NMR}\left(\mathrm{CDCl}_{3}\right) \delta 170.4,146.4$, 143.9, 136.3, 130.4, 128.7, 127.64, 127.58, 127.2, 126.1, 124.7, 56.3, 52.8, 47.5, 45.7, 41.9. TLC: $\mathrm{R}_{\mathrm{f}} 0.36\left(\mathrm{CHCl}_{3} / \mathrm{MeOH}=10: 1\right)$. HRMS Calcd for $\mathrm{C}_{19} \mathrm{H}_{23} \mathrm{~N}_{2} \mathrm{OS}:[\mathrm{M}+\mathrm{H}]^{+}$, 327.1526. Found: $m / z$ 327.1521. HPLC (Daicel Chiralpak IE, hexane $/ i-\mathrm{PrOH}=80 / 20$, flow rate $\left.=2.0 \mathrm{~mL} / \mathrm{min}, \lambda=254 \mathrm{~nm}, 30^{\circ} \mathrm{C}\right): t_{\text {major }}=8.0 \mathrm{~min}, t_{\text {minor }}=9.8 \mathrm{~min}$.

The absolute configuration of $\mathbf{8}$ was assigned as $(R)$ by comparing the optical rotation with the literature value. ${ }^{9}$

$[\alpha]_{\mathrm{D}}{ }^{18}-416.7$ ( $c$ 0.33, $\mathrm{CHCl}_{3}$ ) [lit. ${ }^{9}(R)$-5-(2-(dimethylamino)ethyl)-2-phenyl-2,3dihydrobenzo[b][1,4]thiazepin-4(5H)-one $(99 \% e e)$ : $\left.[\alpha]_{\mathrm{D}}{ }^{18}-468.3\left(c 0.36, \mathrm{CHCl}_{3}\right)\right]$. 


\section{References}

1. Birman, V. B.; Li, X. Org. Lett. 2008, 10, 1115.

2. Oyamada, J.; Kitamura, T. Chem. Lett. 2005, 34, 1430.

3. Mizukami, S.; Kono, M. Chem. Pharm. Bull. 1965, 13, 33.

4. Ayers, J. T.; Anderson, S. R. Synth. Commun. 1999, 29, 351.

5. Kirihara, M.; Asai, Y.; Ogawa, S.; Noguchi, T.; Hatano, A.; Hirai, Y. Synthesis 2007, 3286.

6. Manfroni, G.; Meschini, F.; Barreca, M. L.; Leyssen, P.; Samuele, A.; Iraci, N.; Sabatini, S.; Massari, S.; Maga, G.; Neyts, J.; Cecchetti, V. Bioorg. Med. Chem. 2012, 20, 866 .

7. Zhao, B.-L.; Du, D.-M. Org. Biomol. Chem. 2014, 12, 1585.

8. Ankner, T.; Hilmersson, G. Org. Lett. 2009, 11, 503.

9. Fang, X.; Li, J.; Wang, C.-J. Org. Lett. 2013, 15, 3448. 
NMR Spectra $\left({ }^{1} \mathrm{H},{ }^{13} \mathrm{C}\right)$ of Products

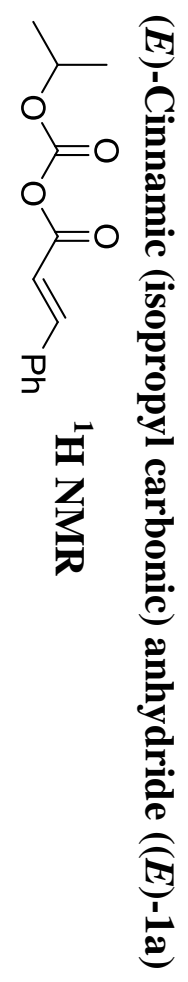

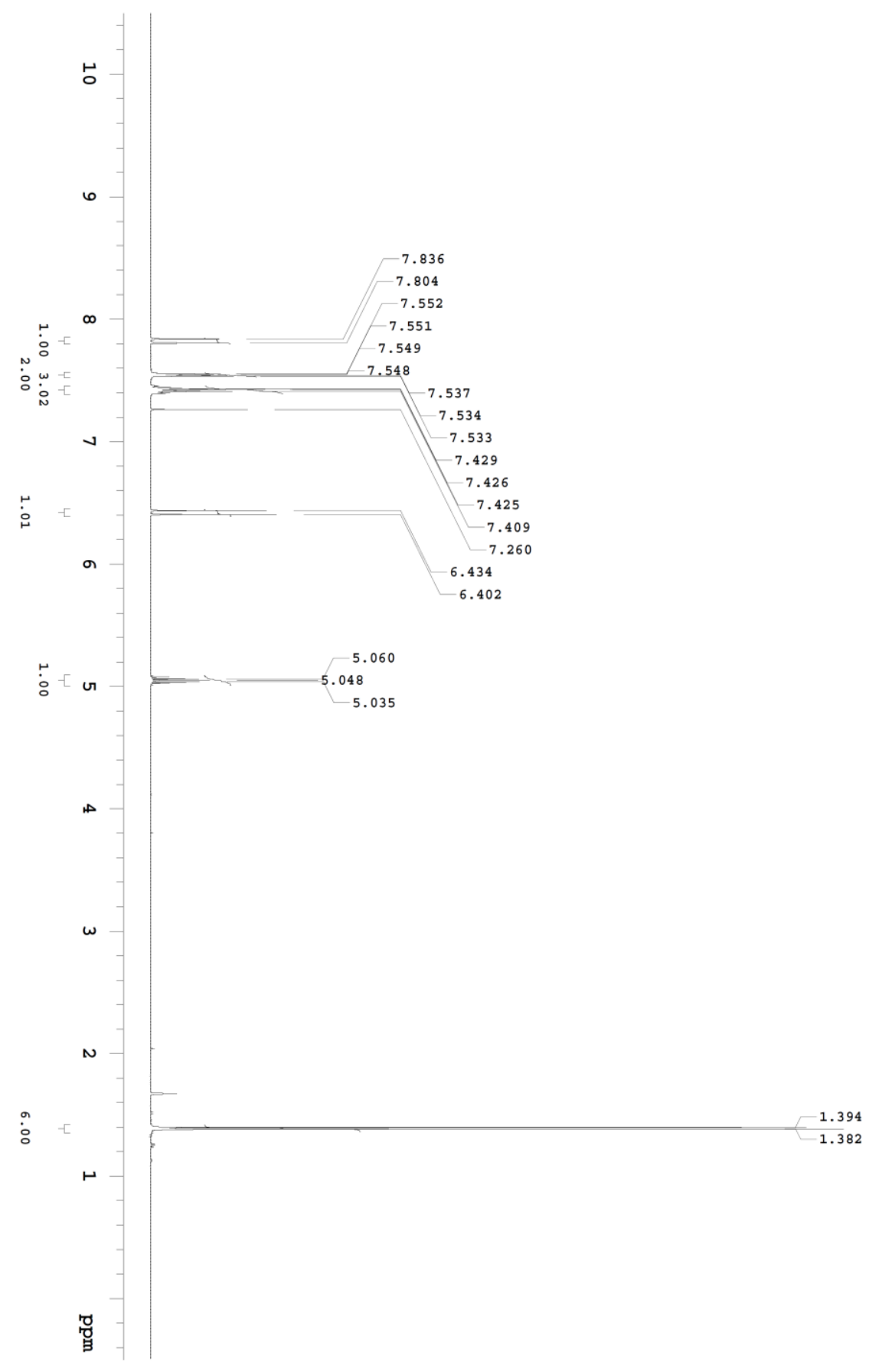




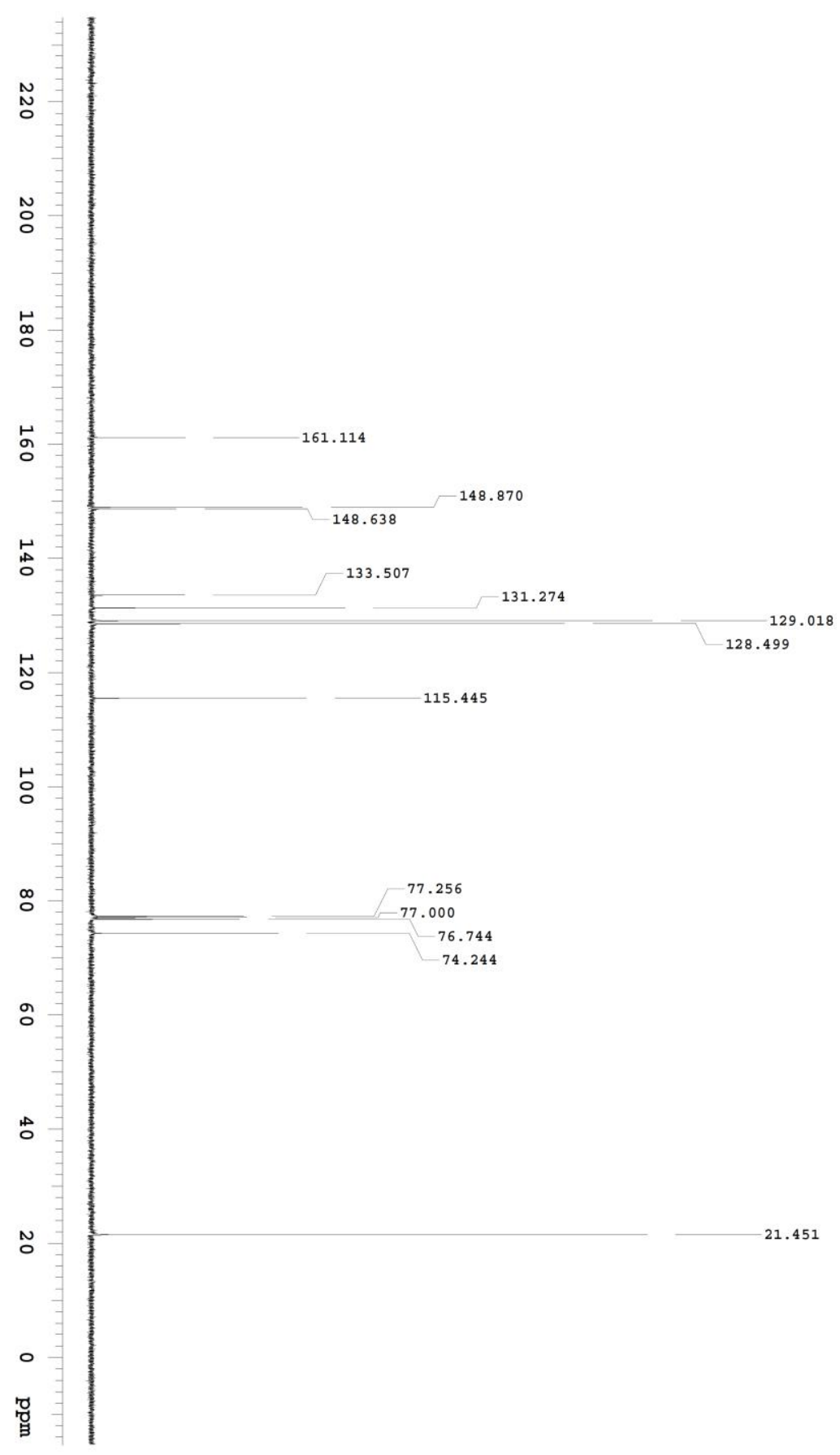

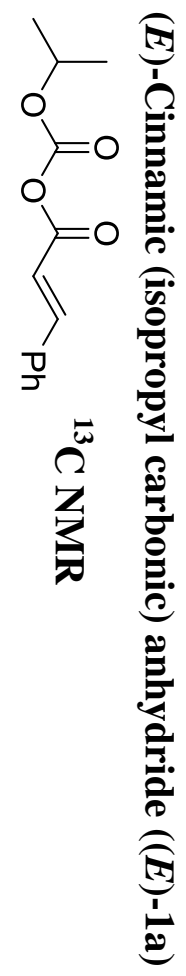



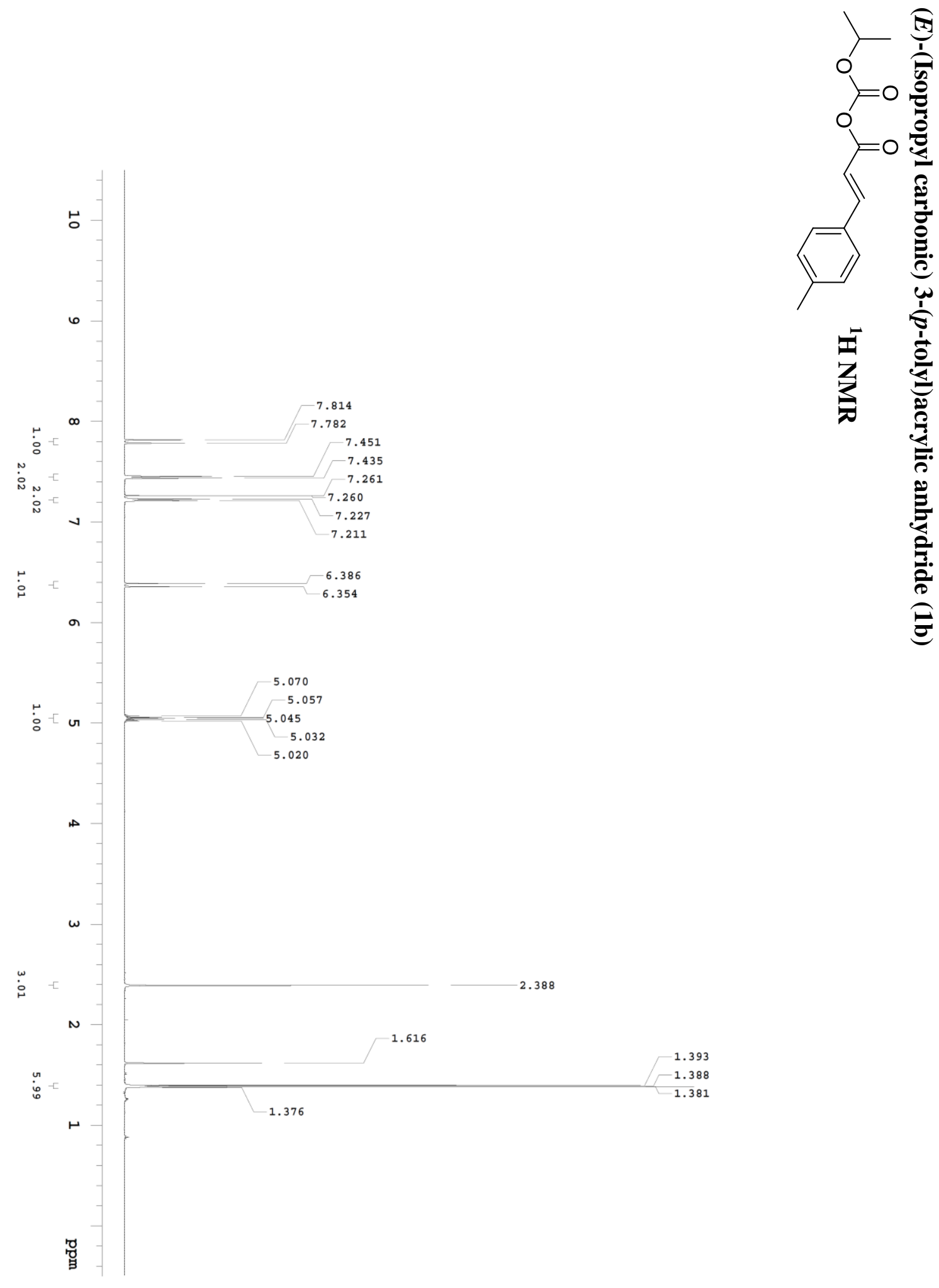

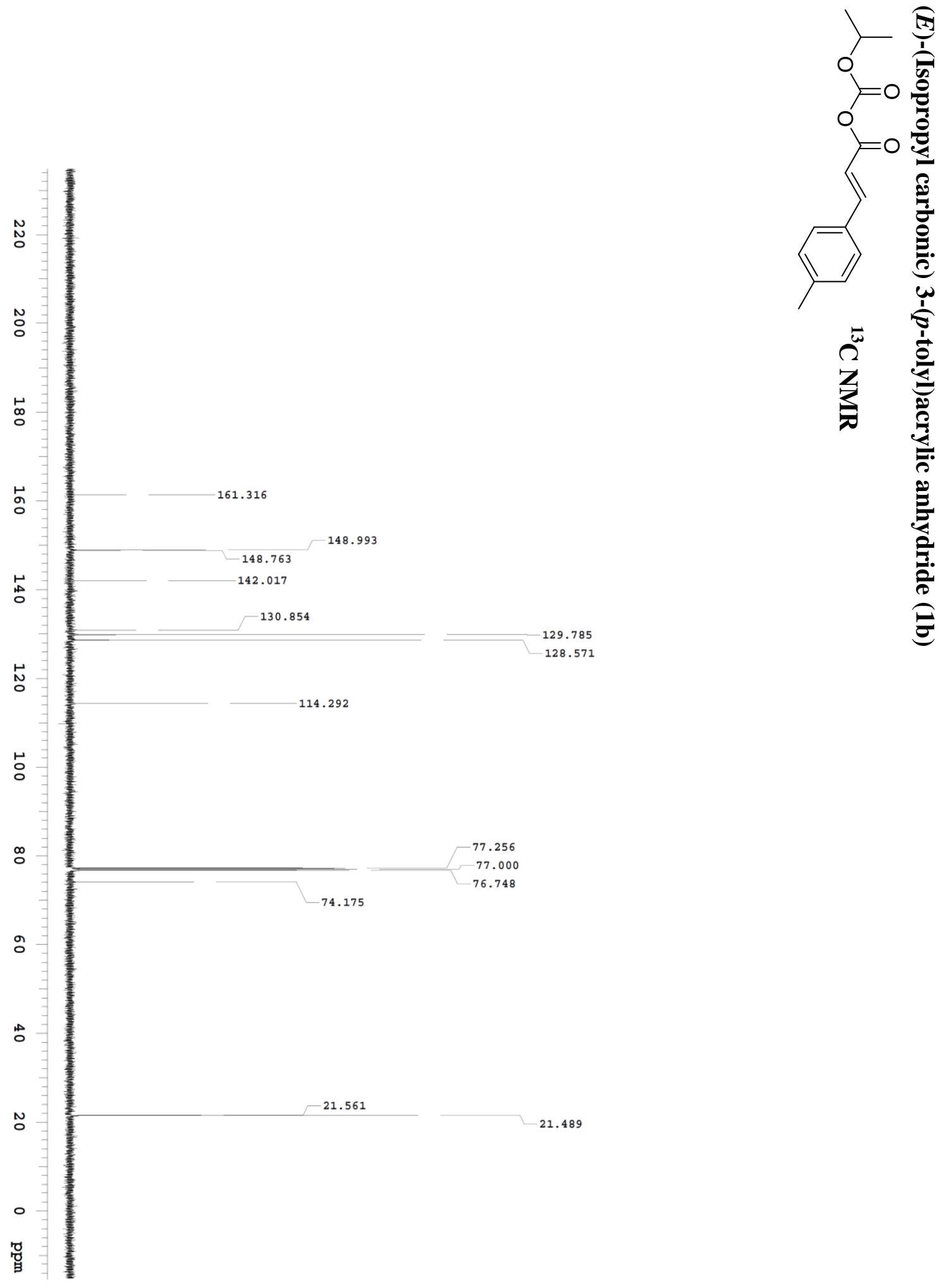

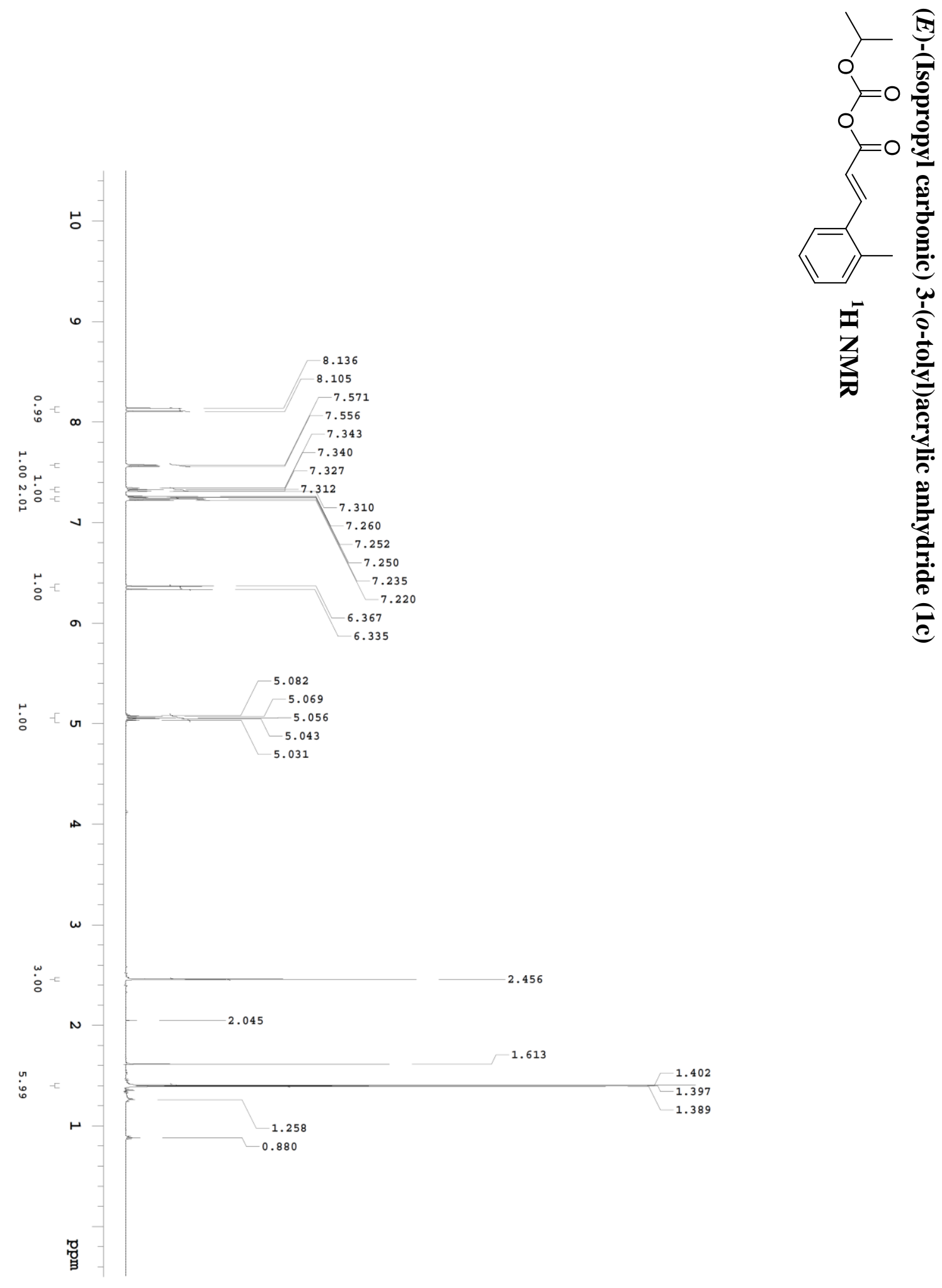

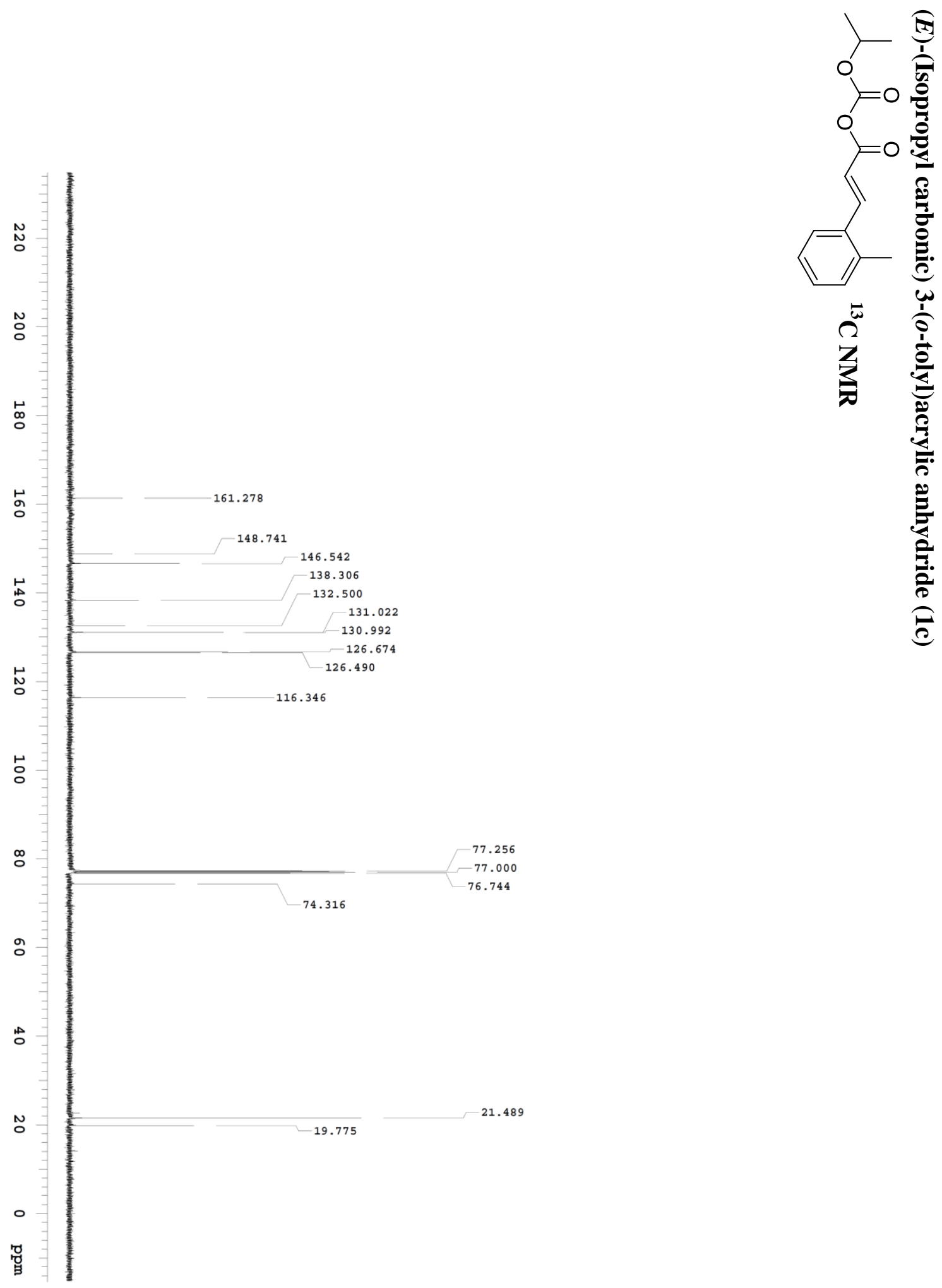

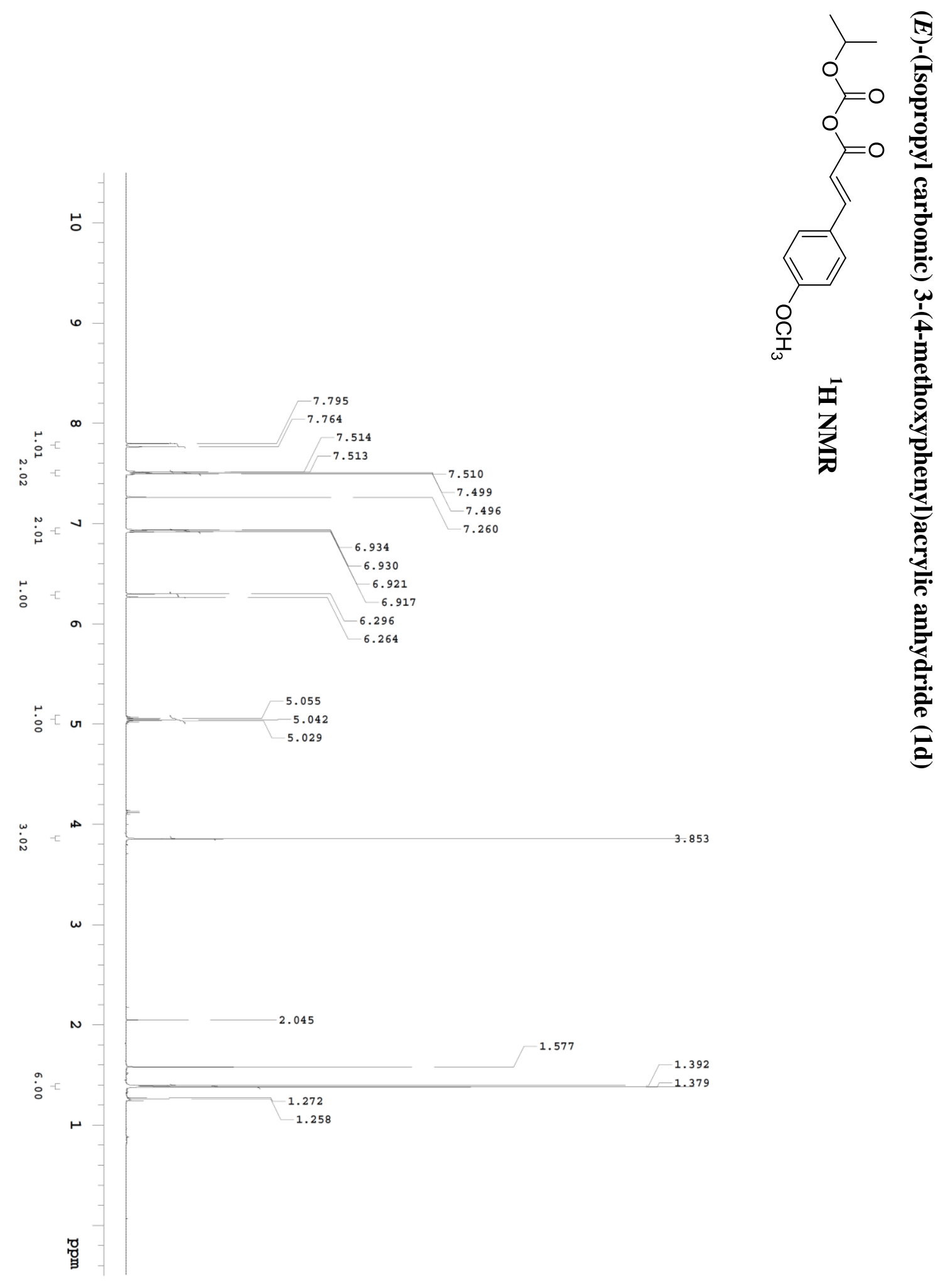

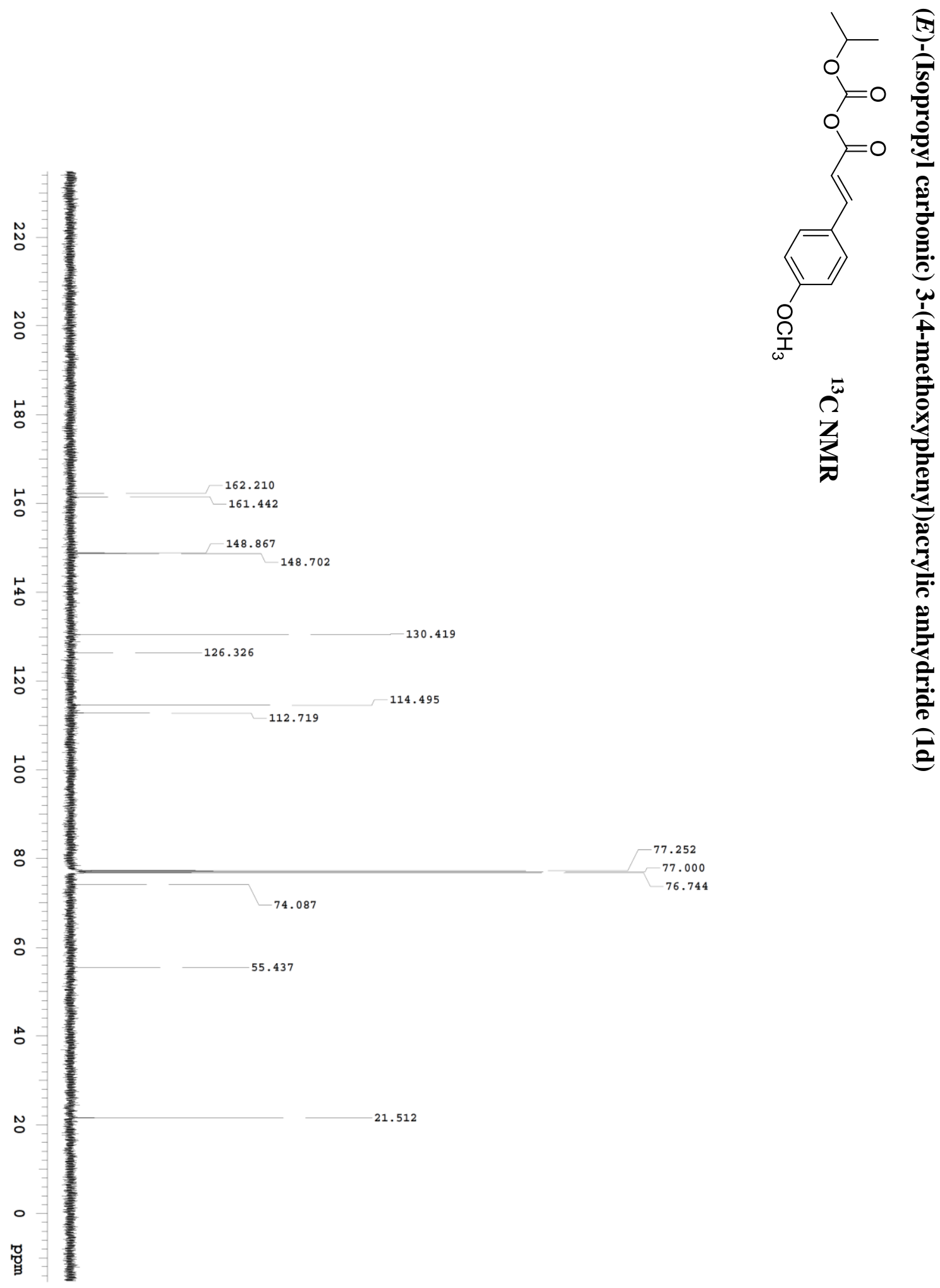

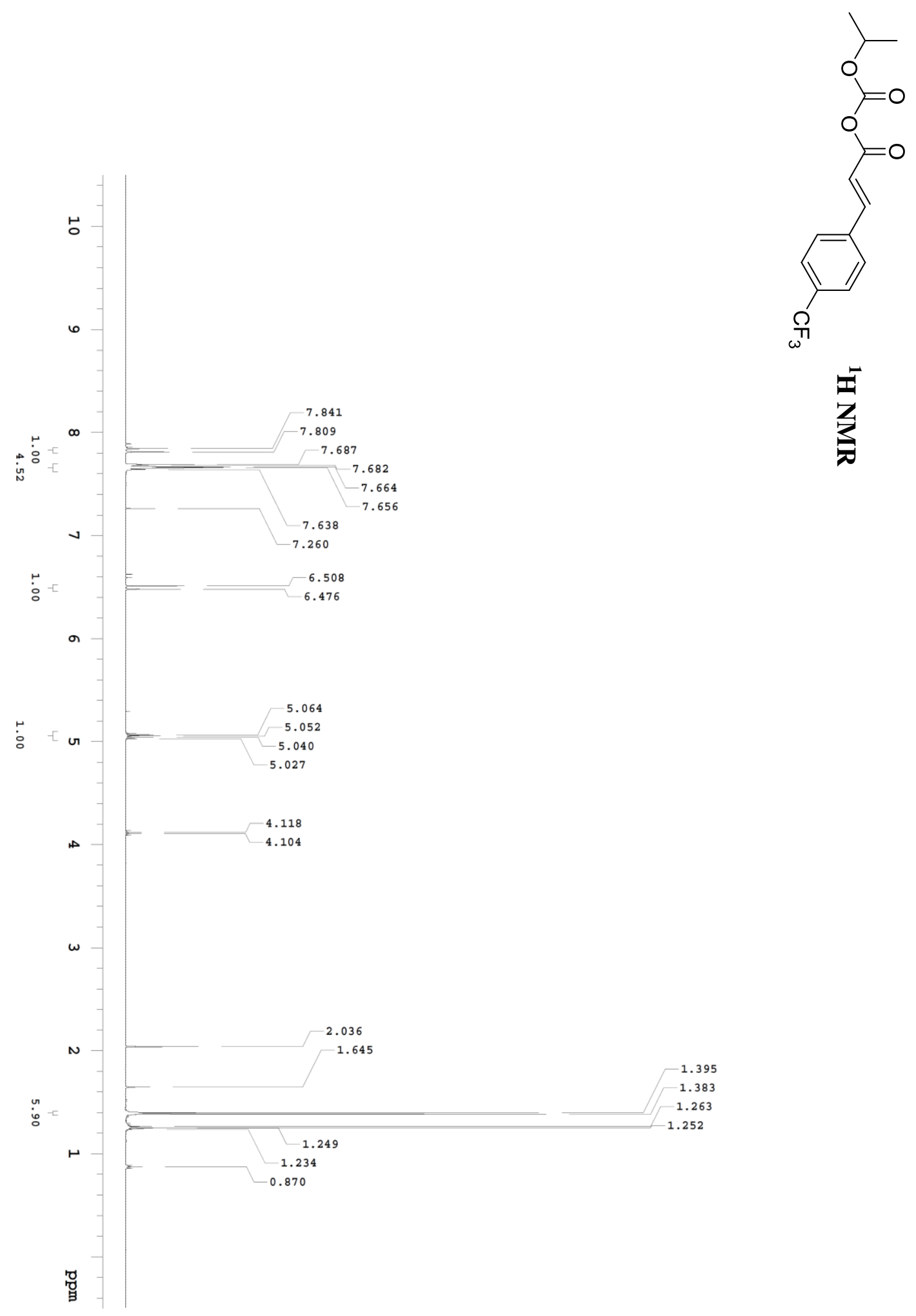

لَّ.

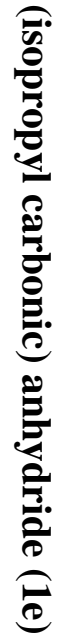



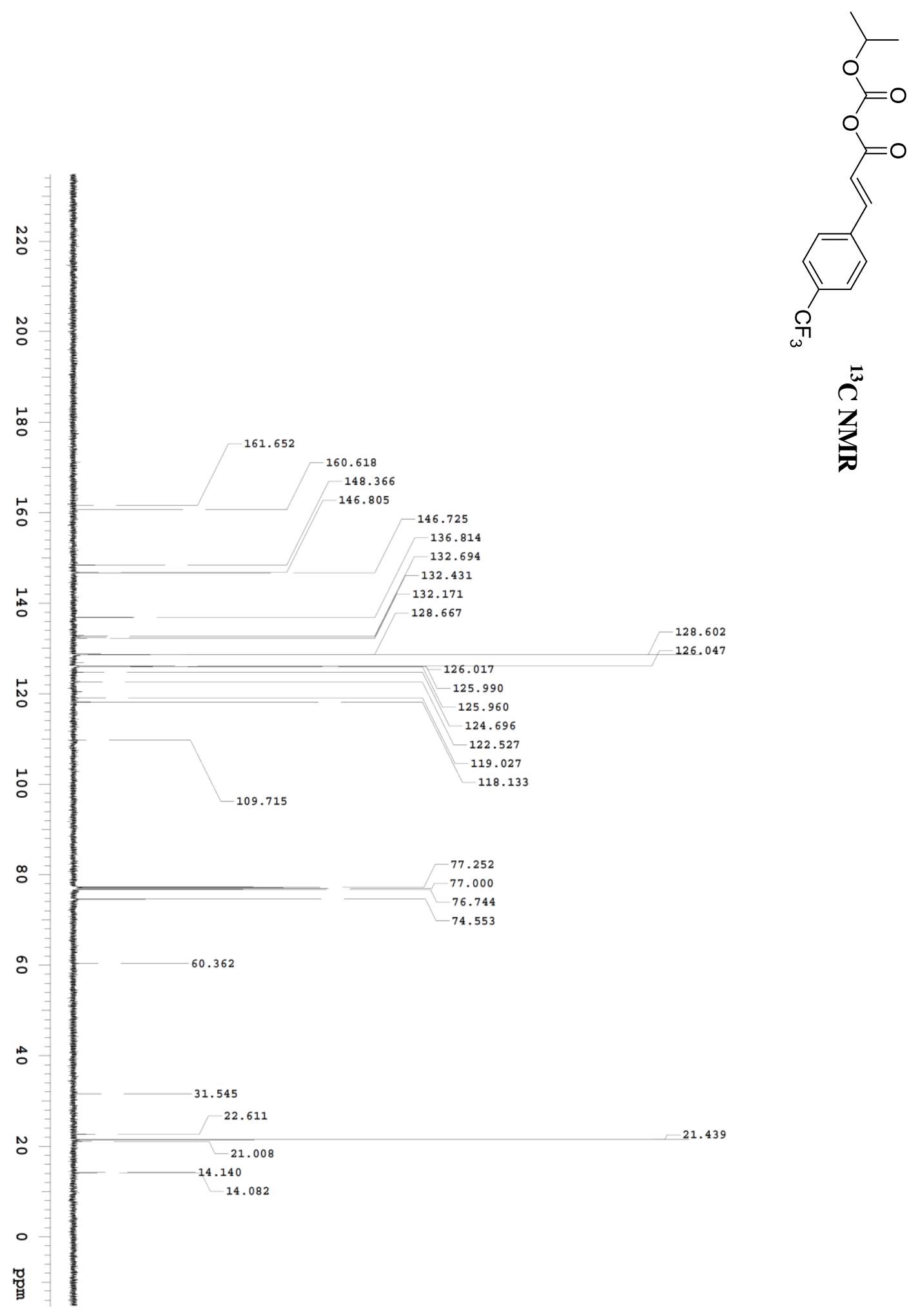

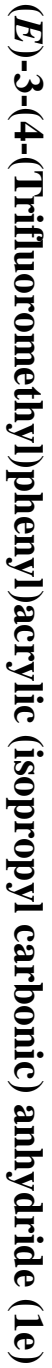



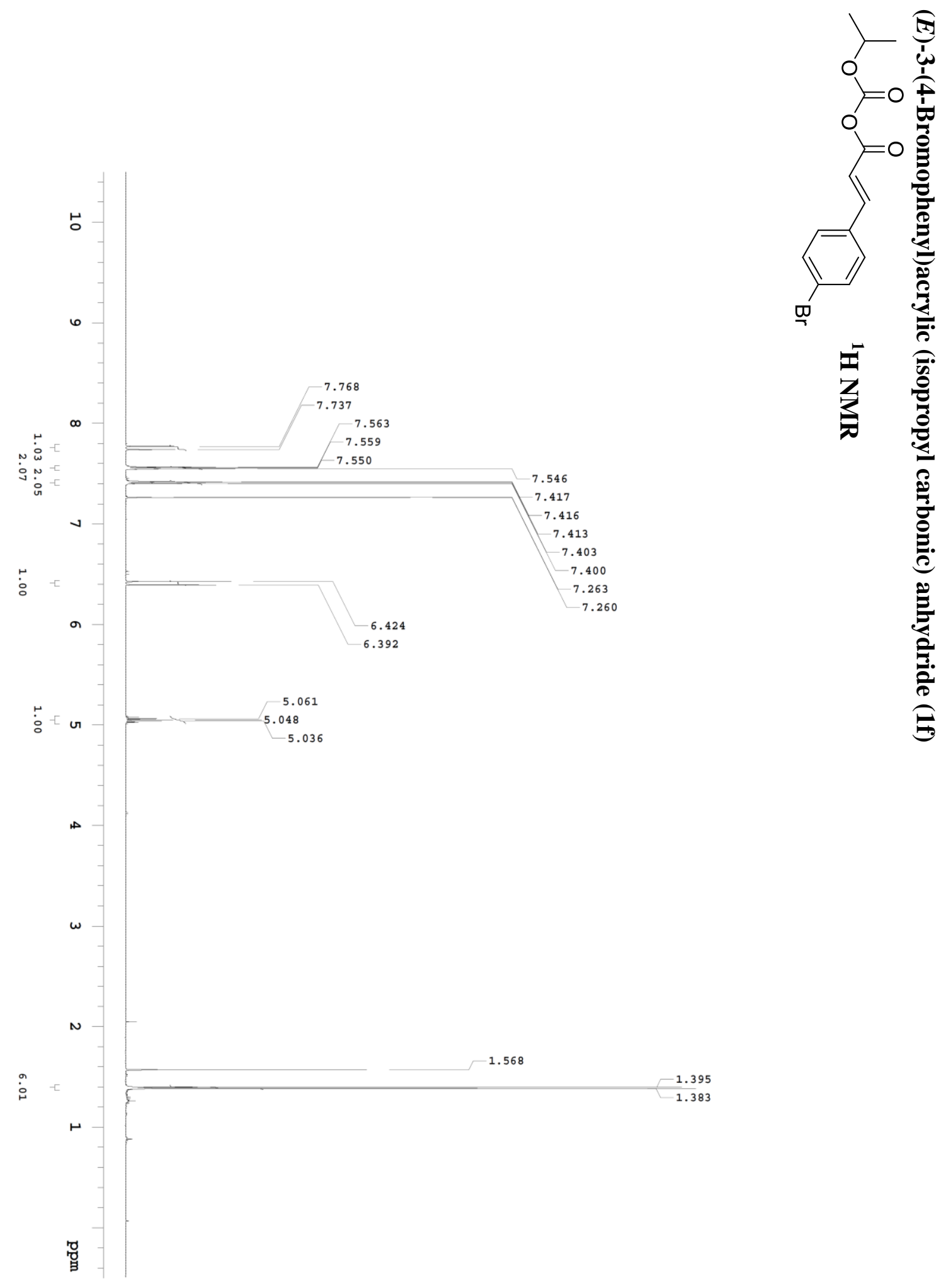

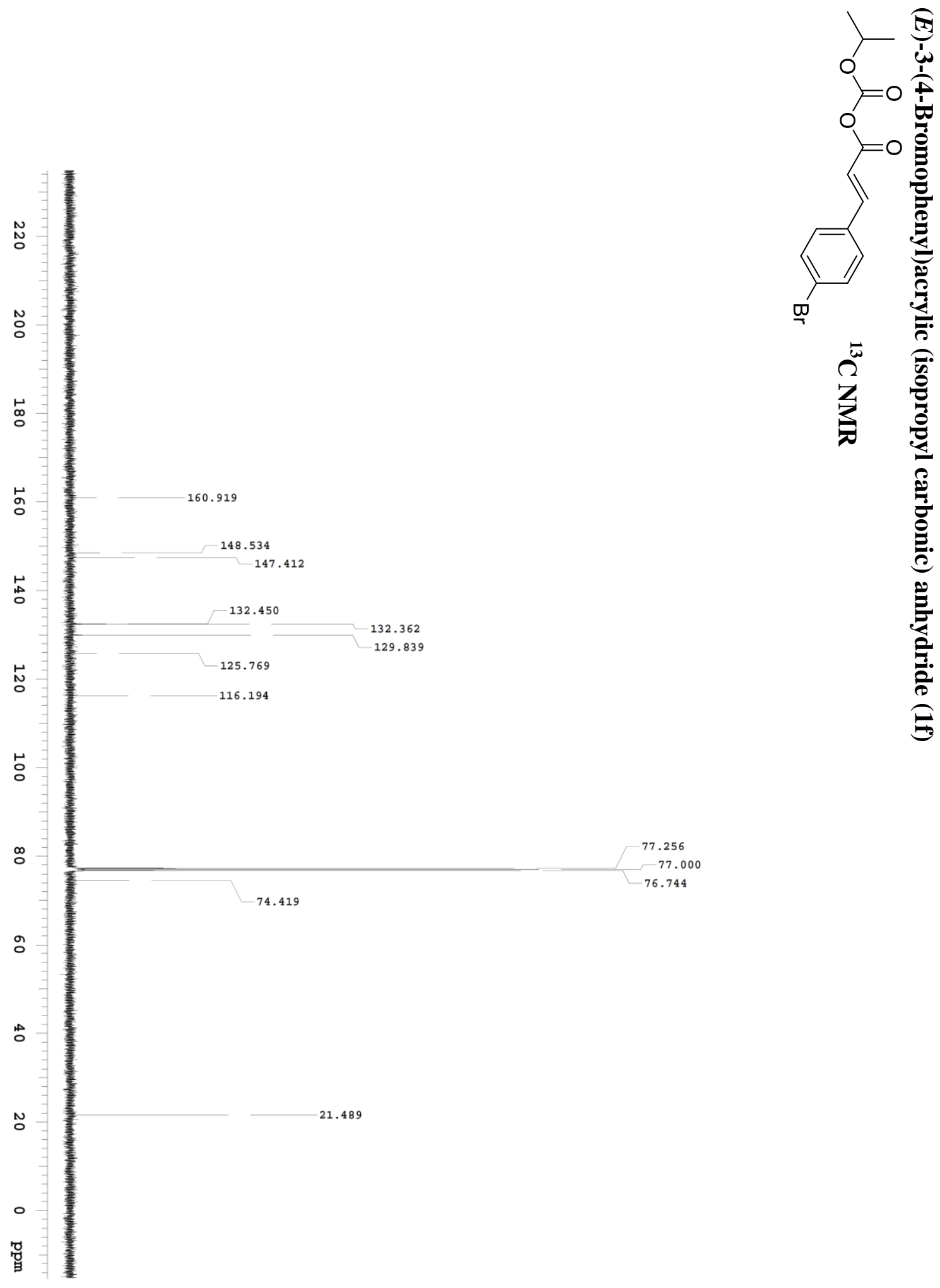

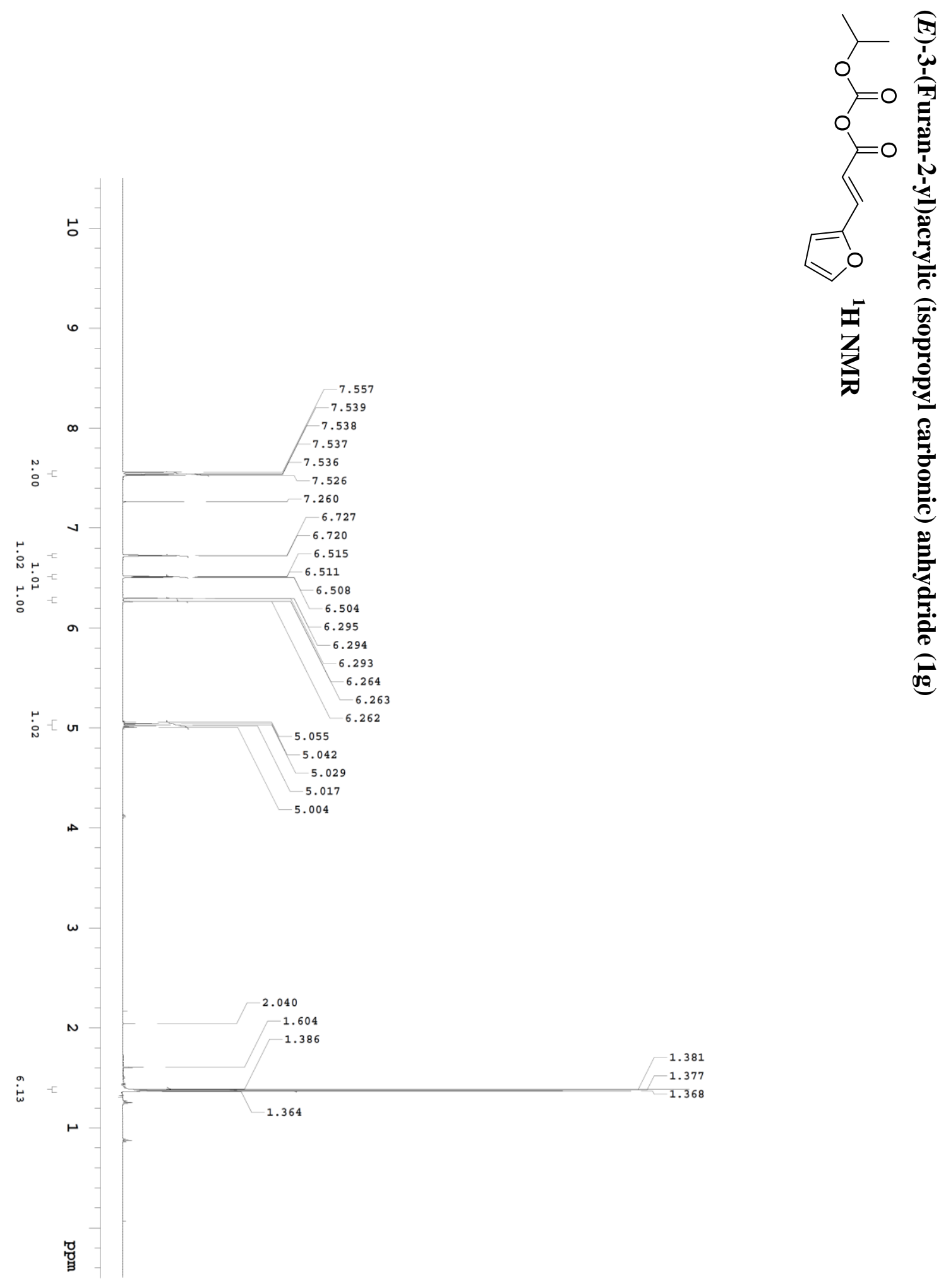

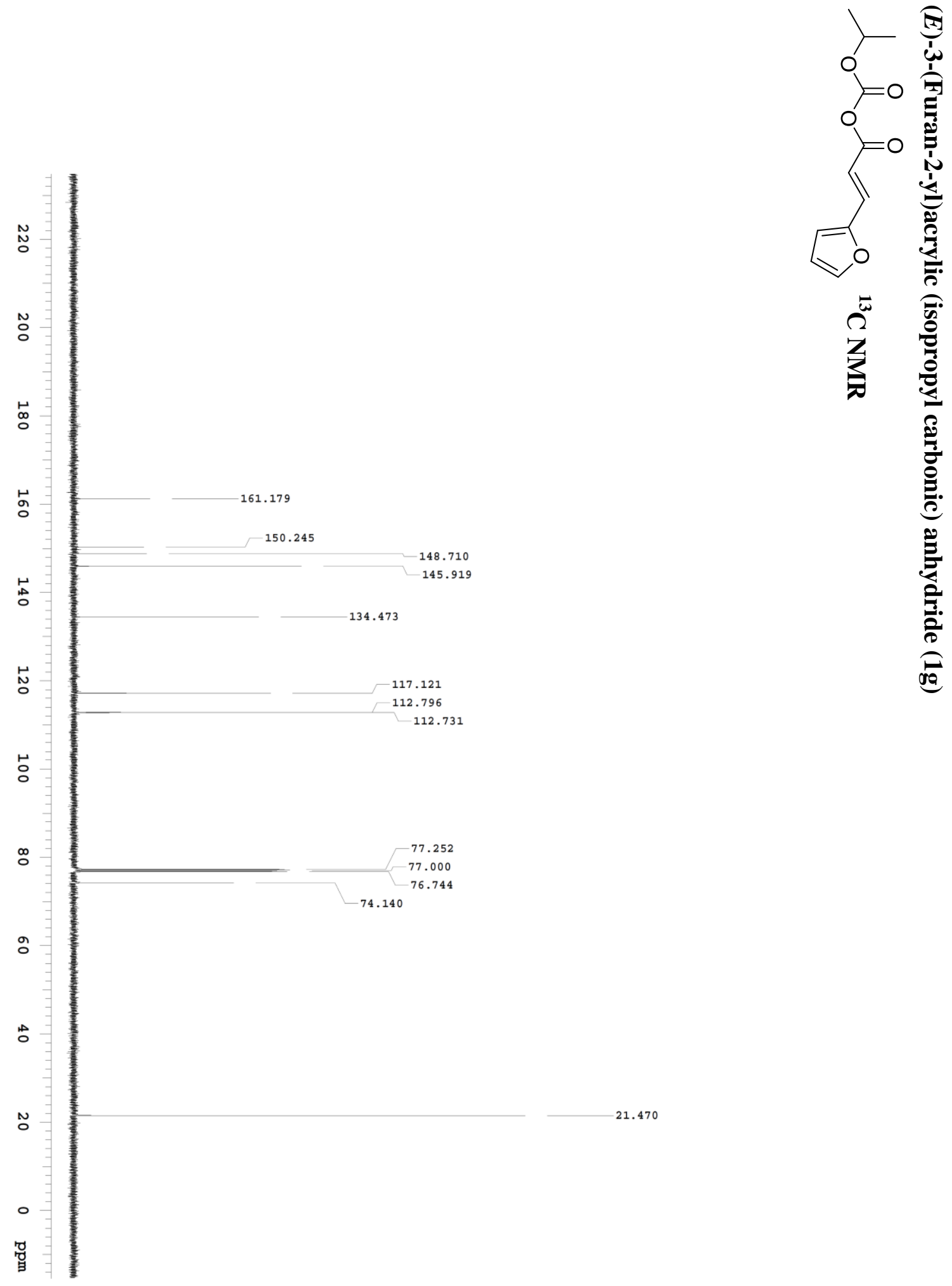


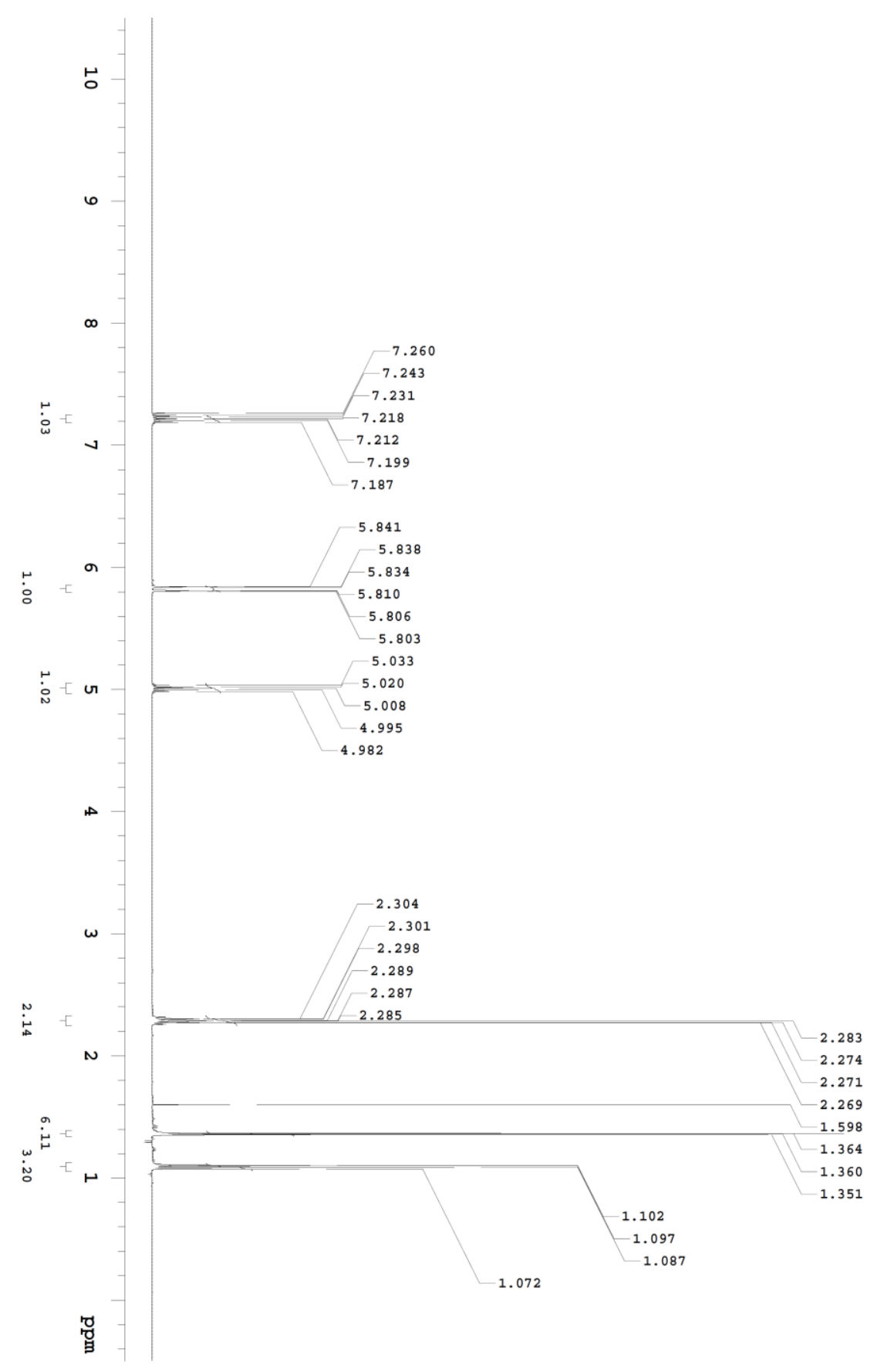

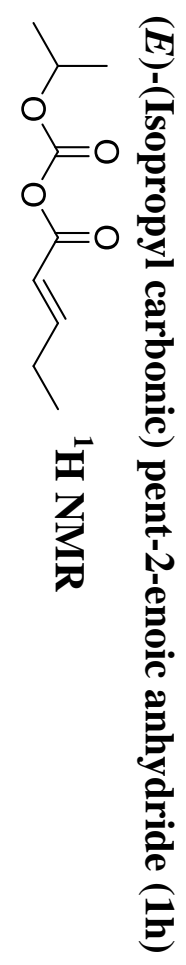




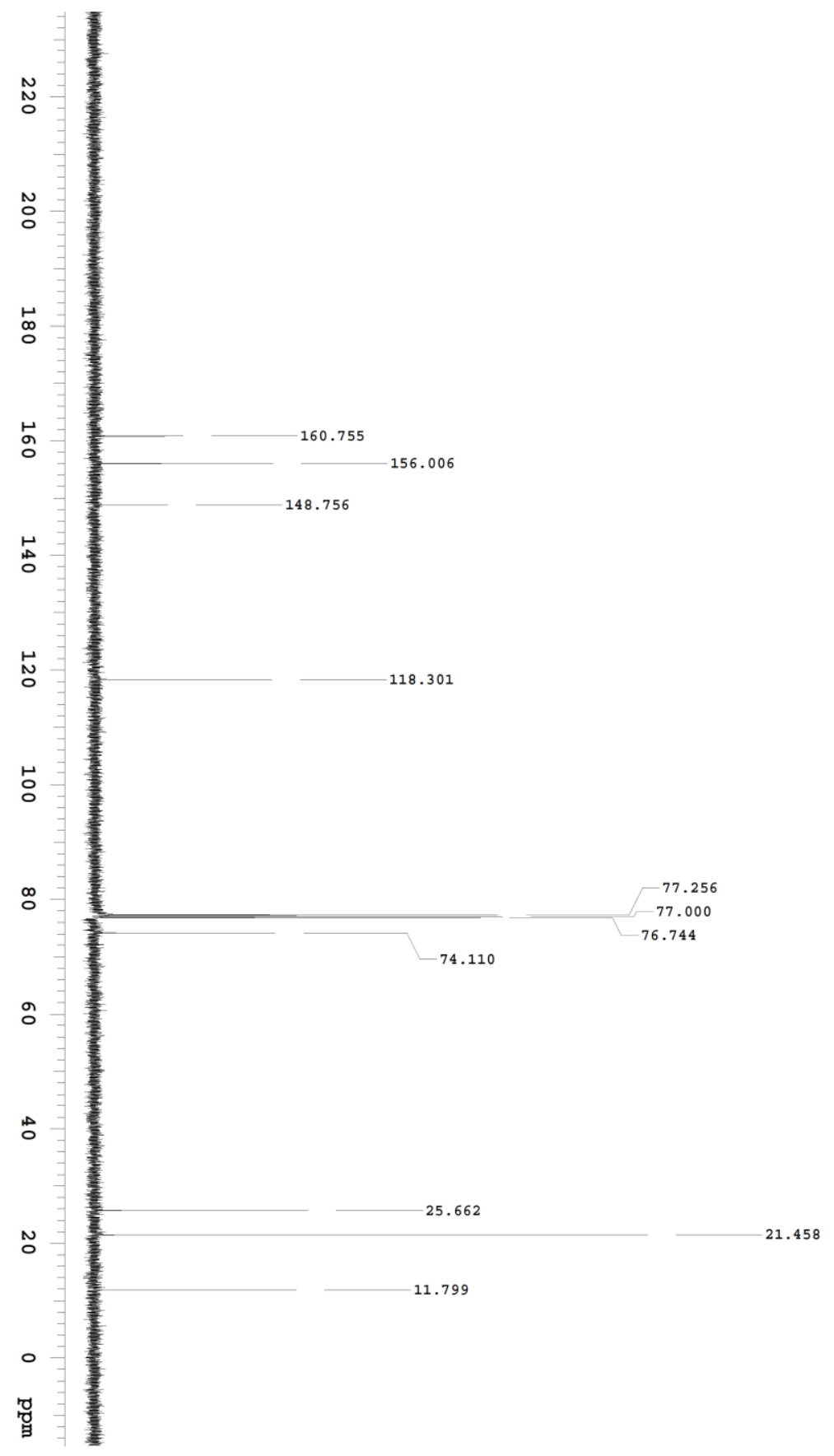

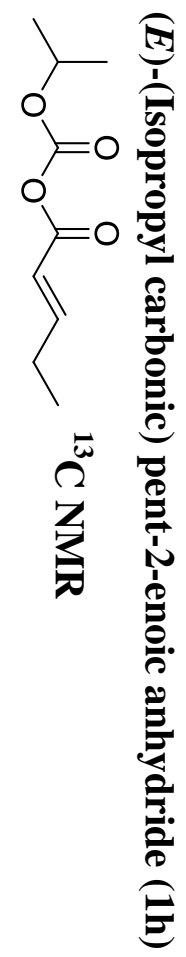



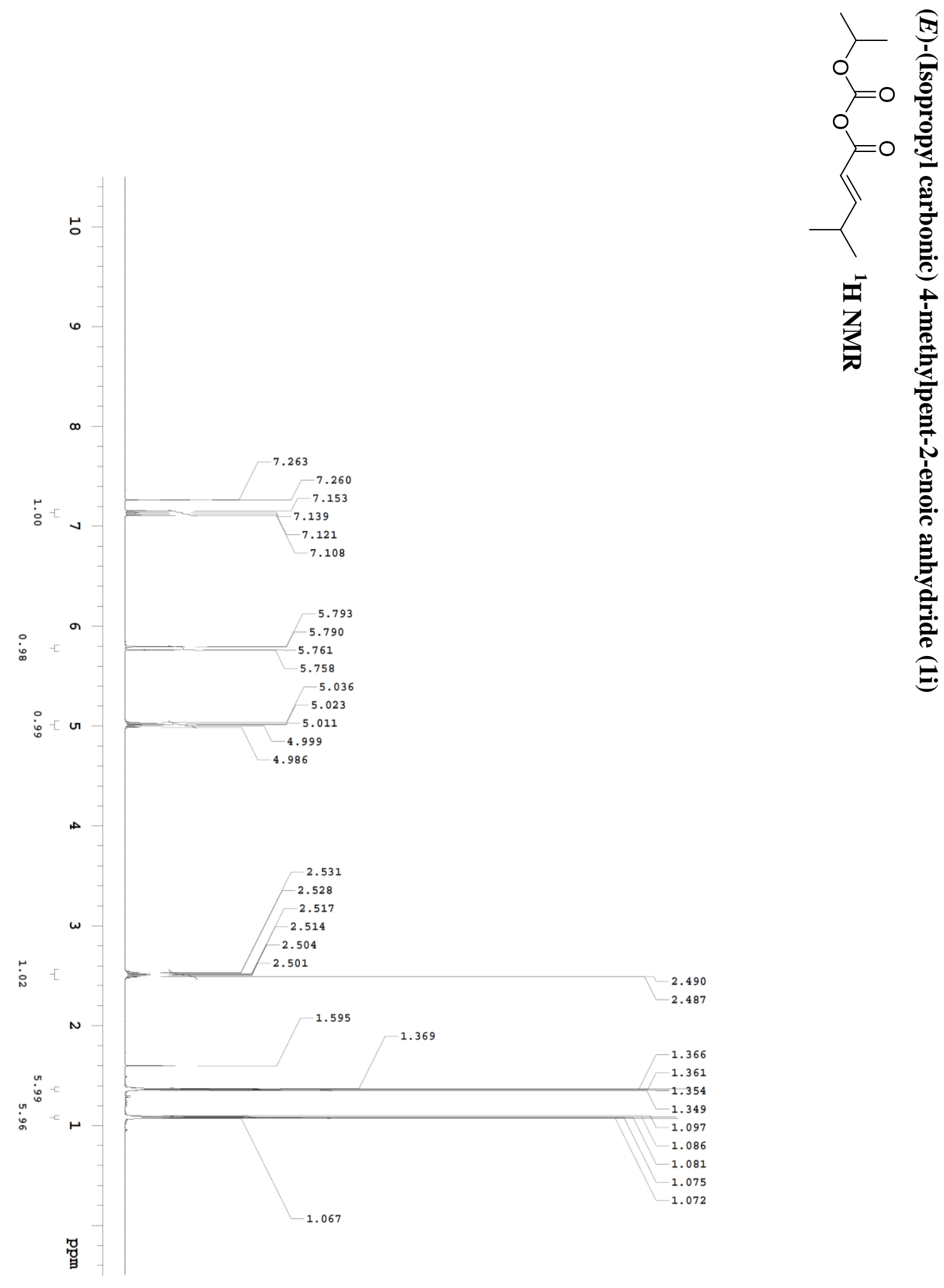

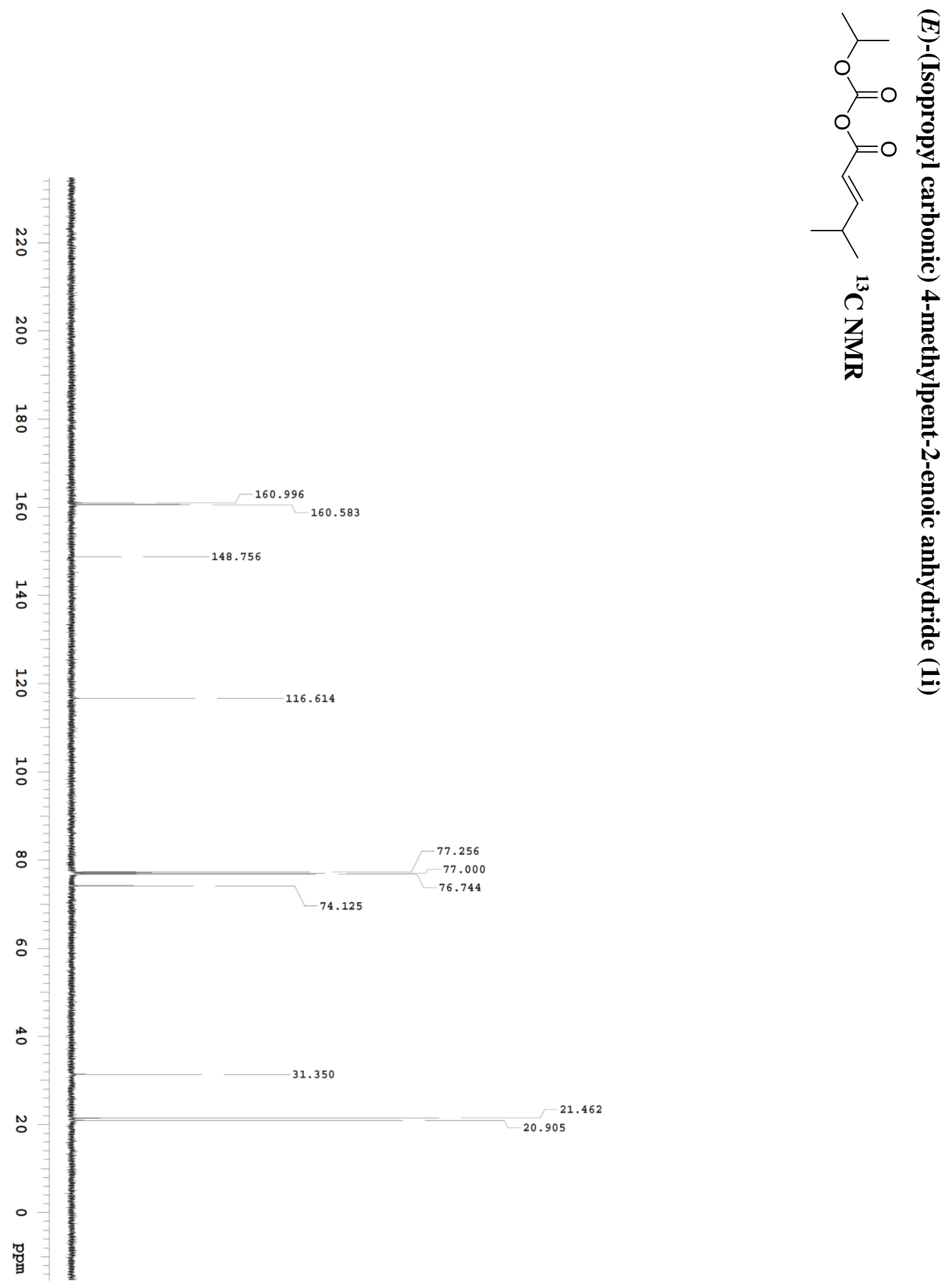

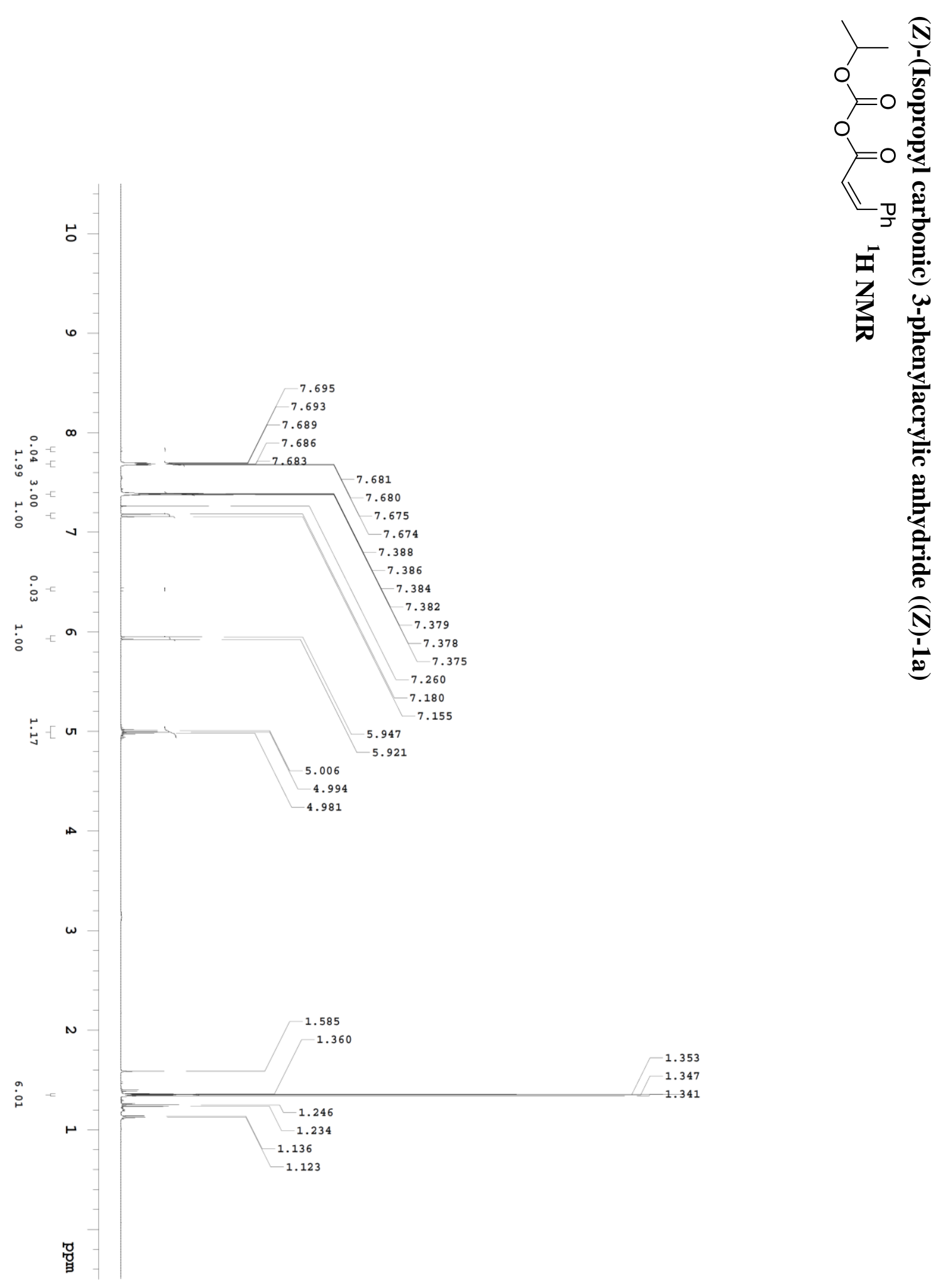


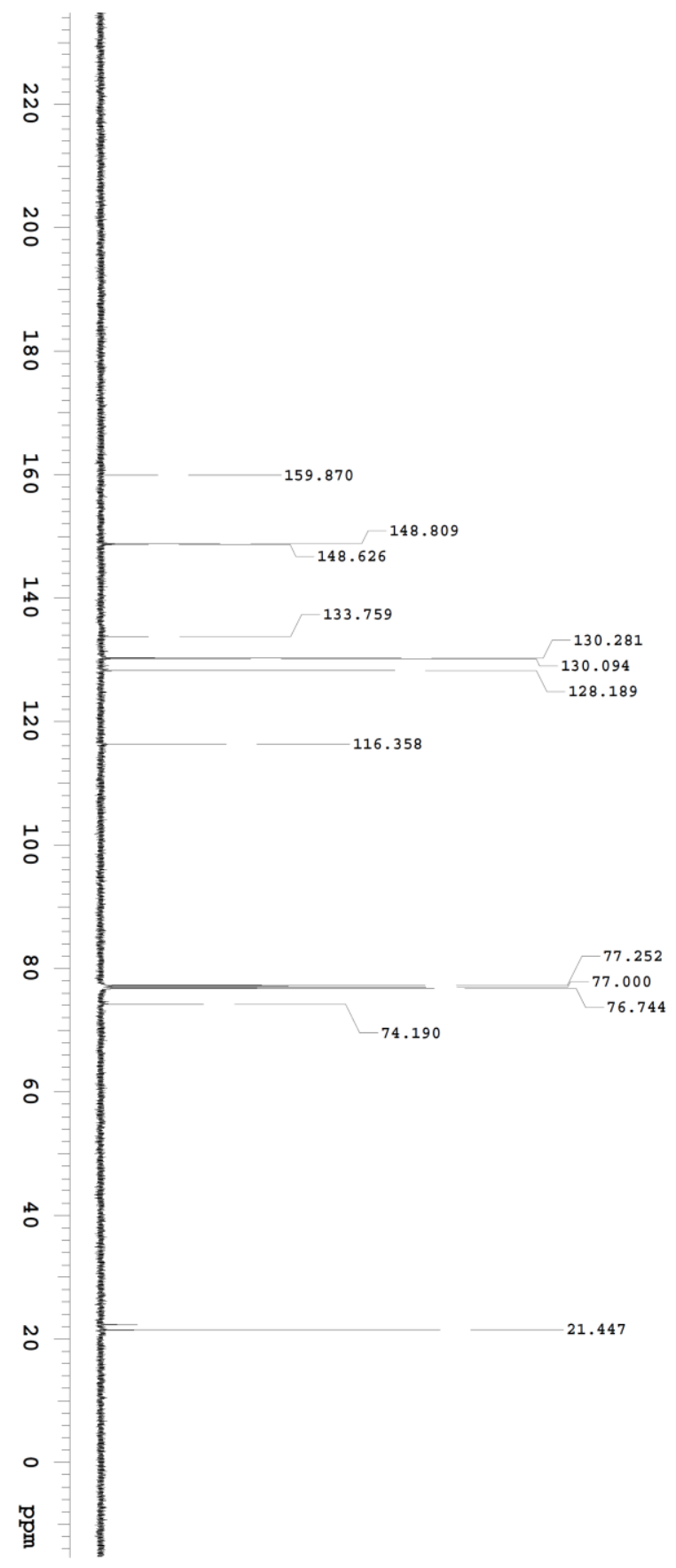

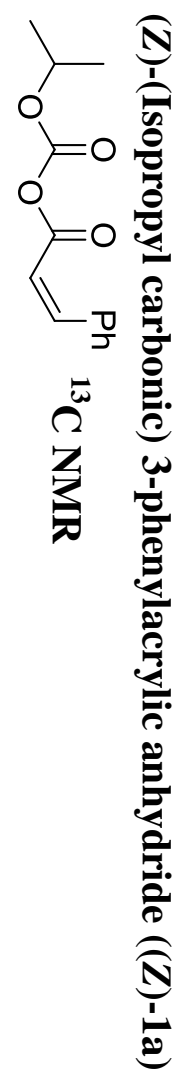




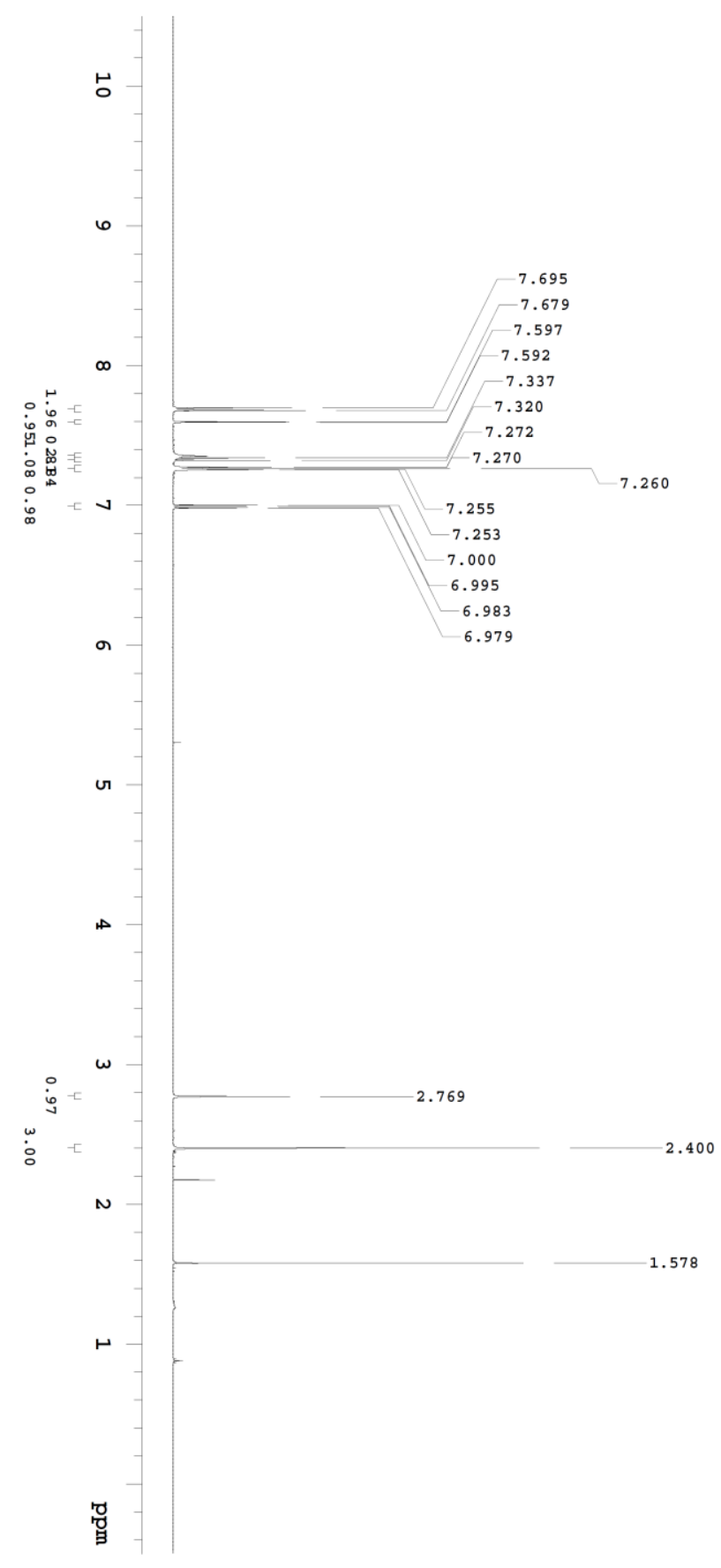

焉



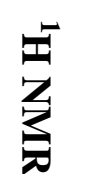



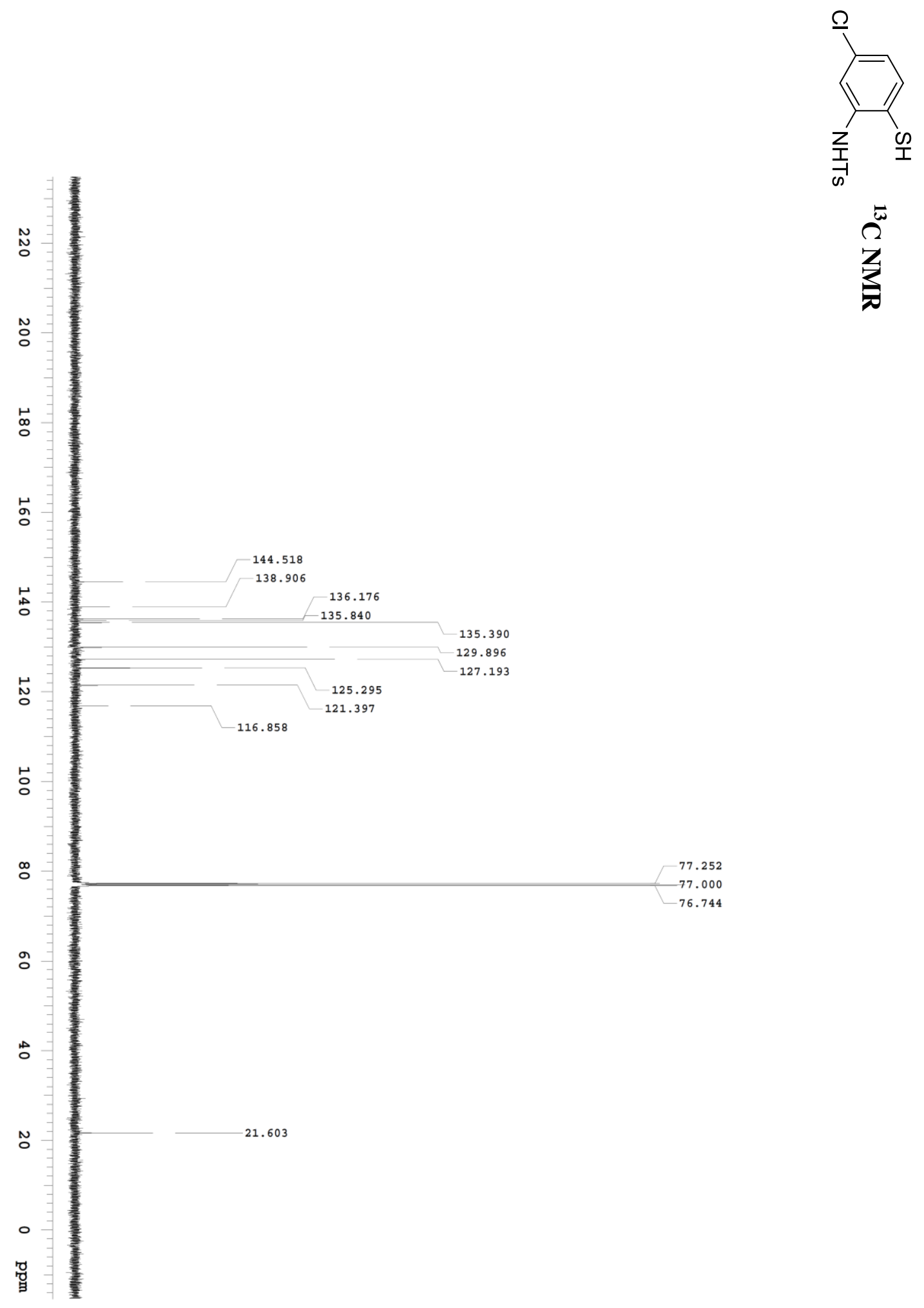

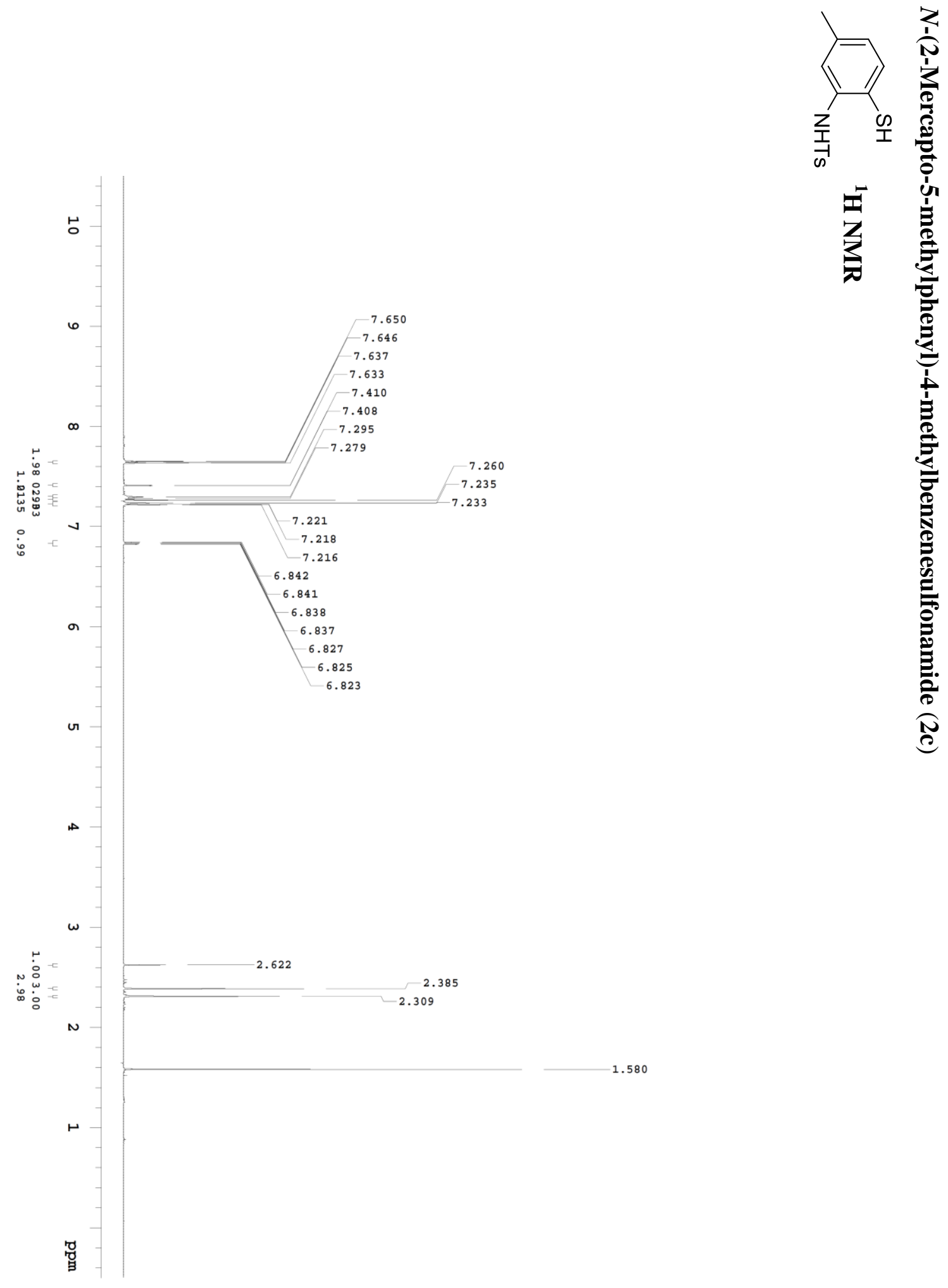

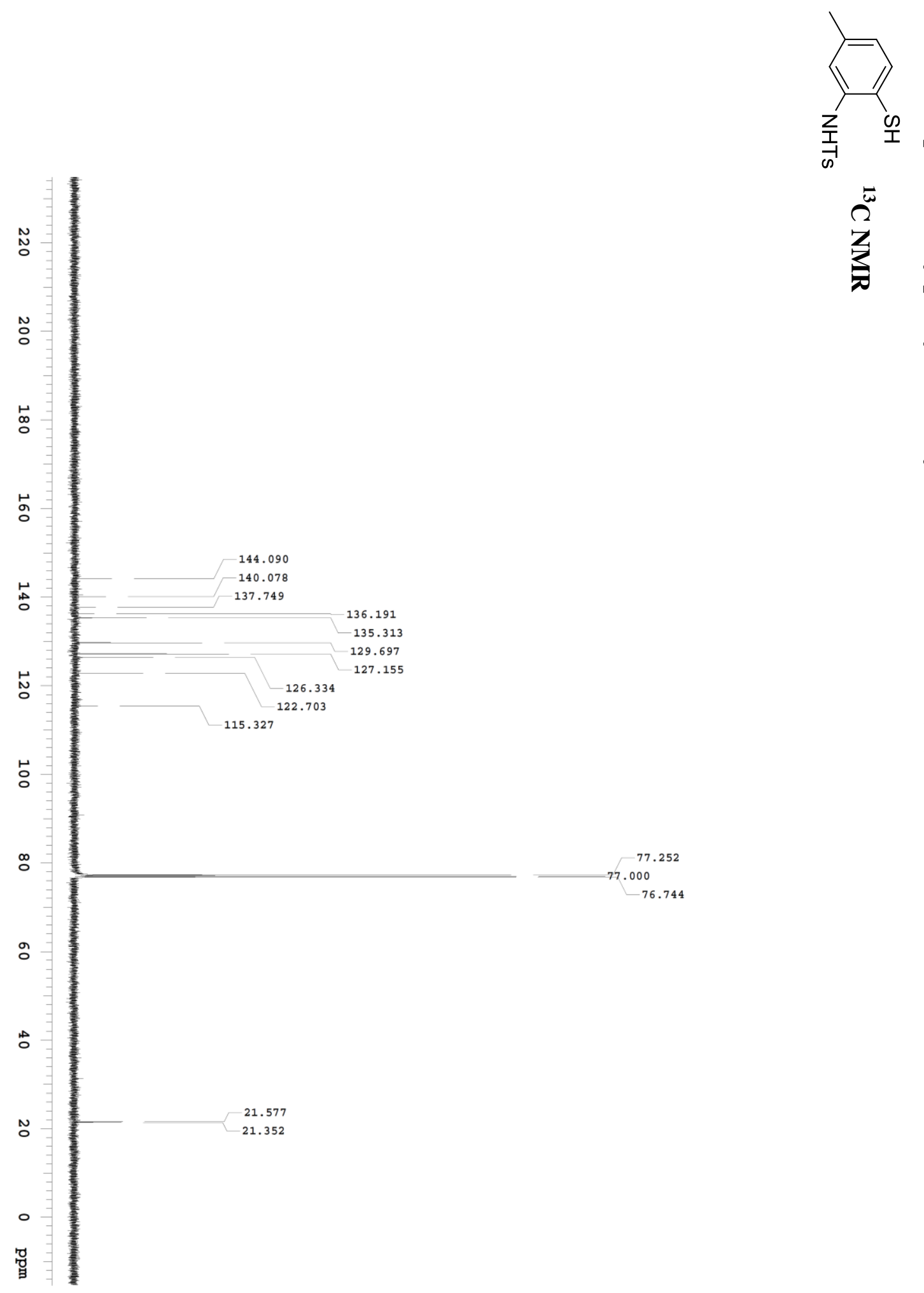

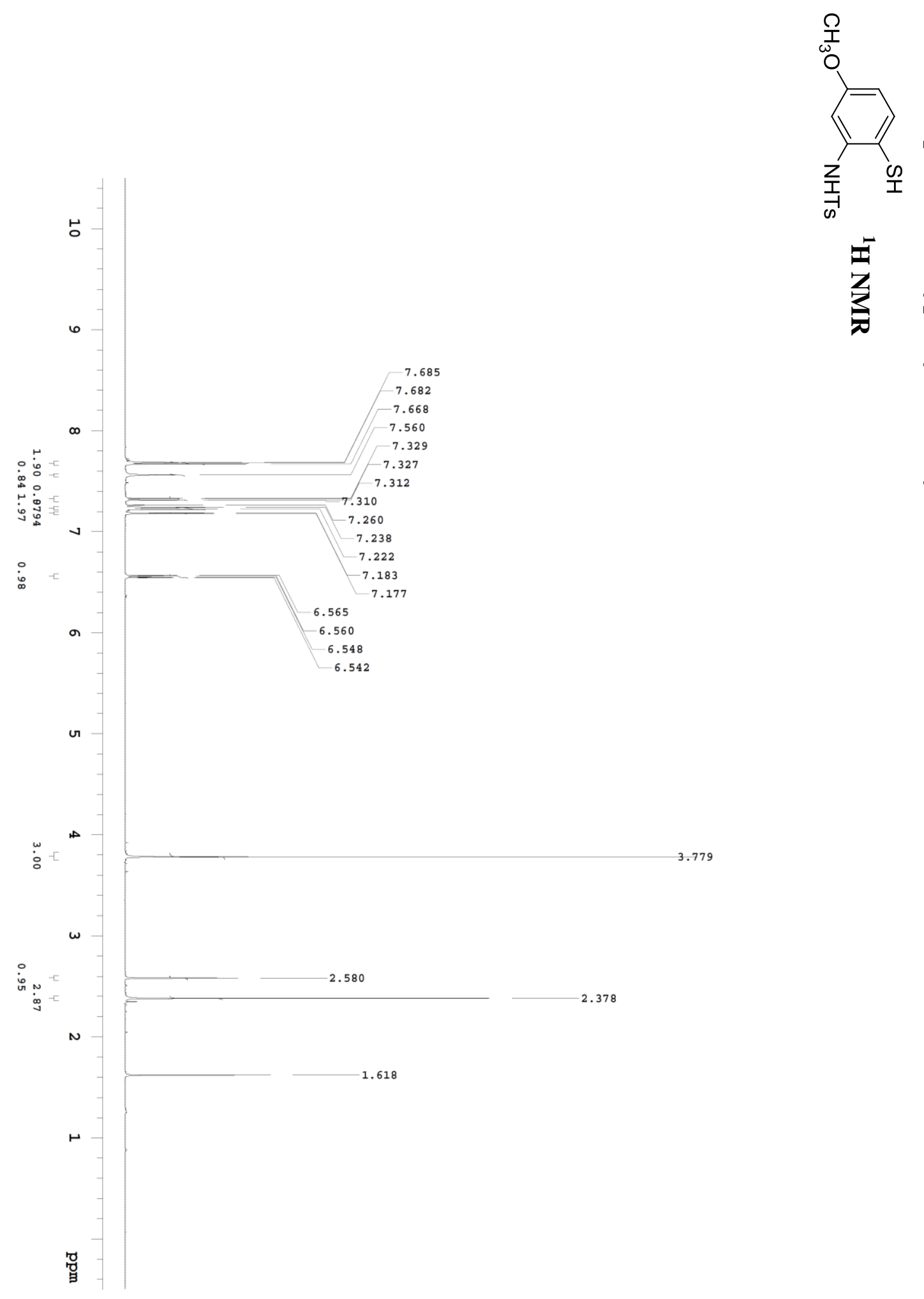

年

竞 

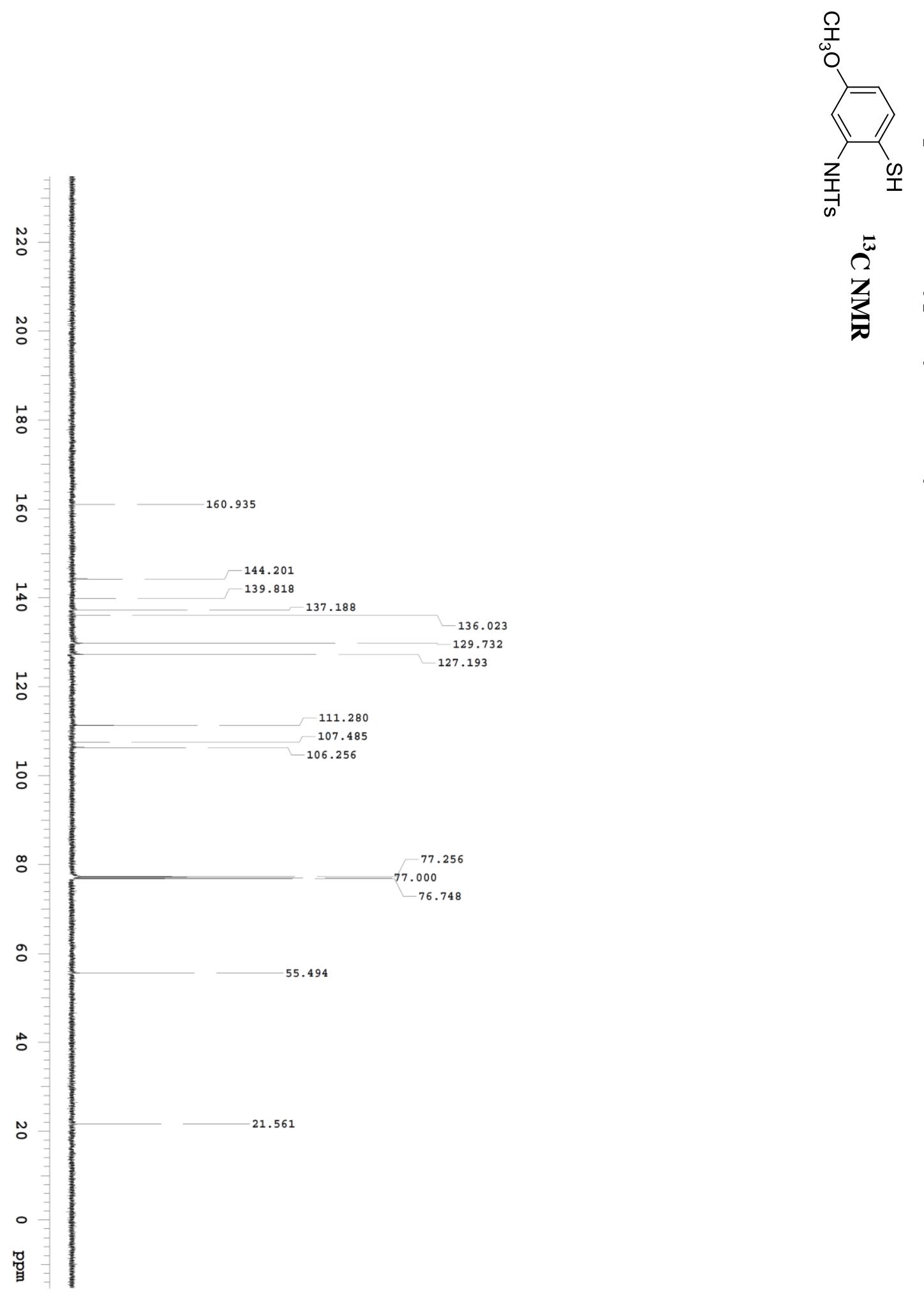

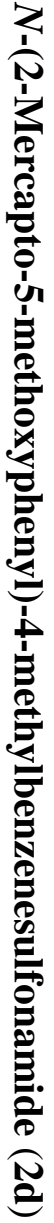




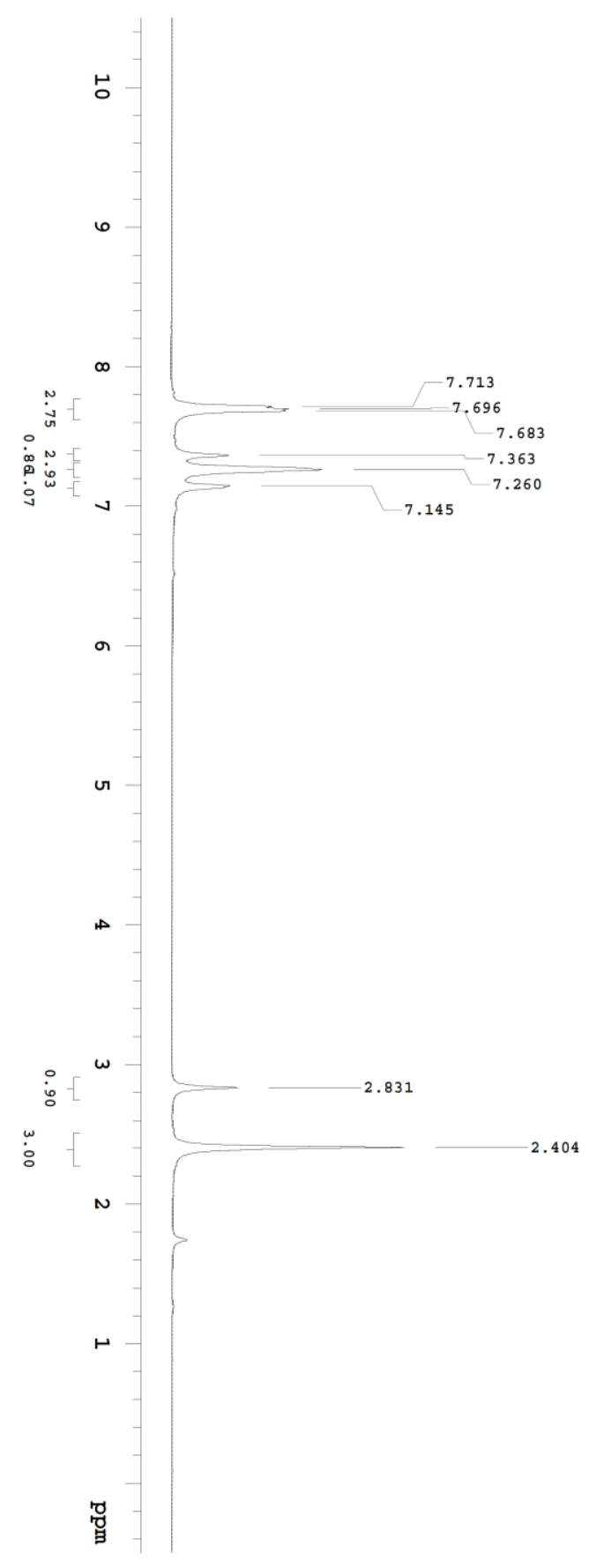

垩 紊

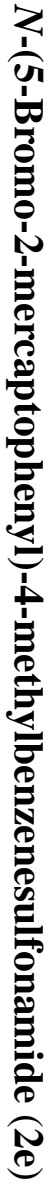




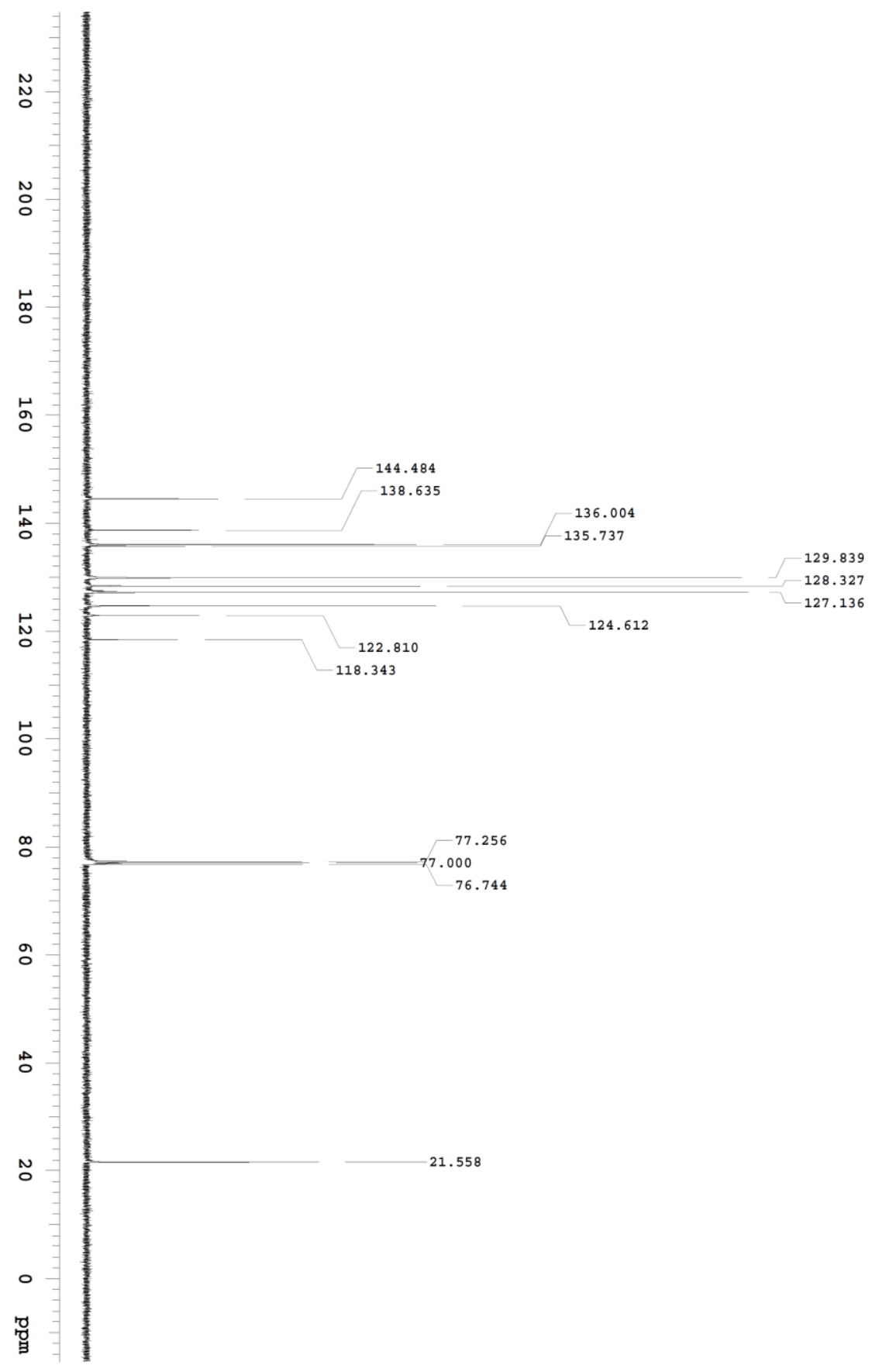

$=$

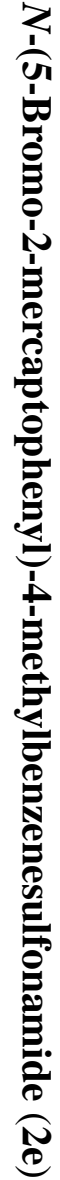

$\overbrace{}^{2}$ 


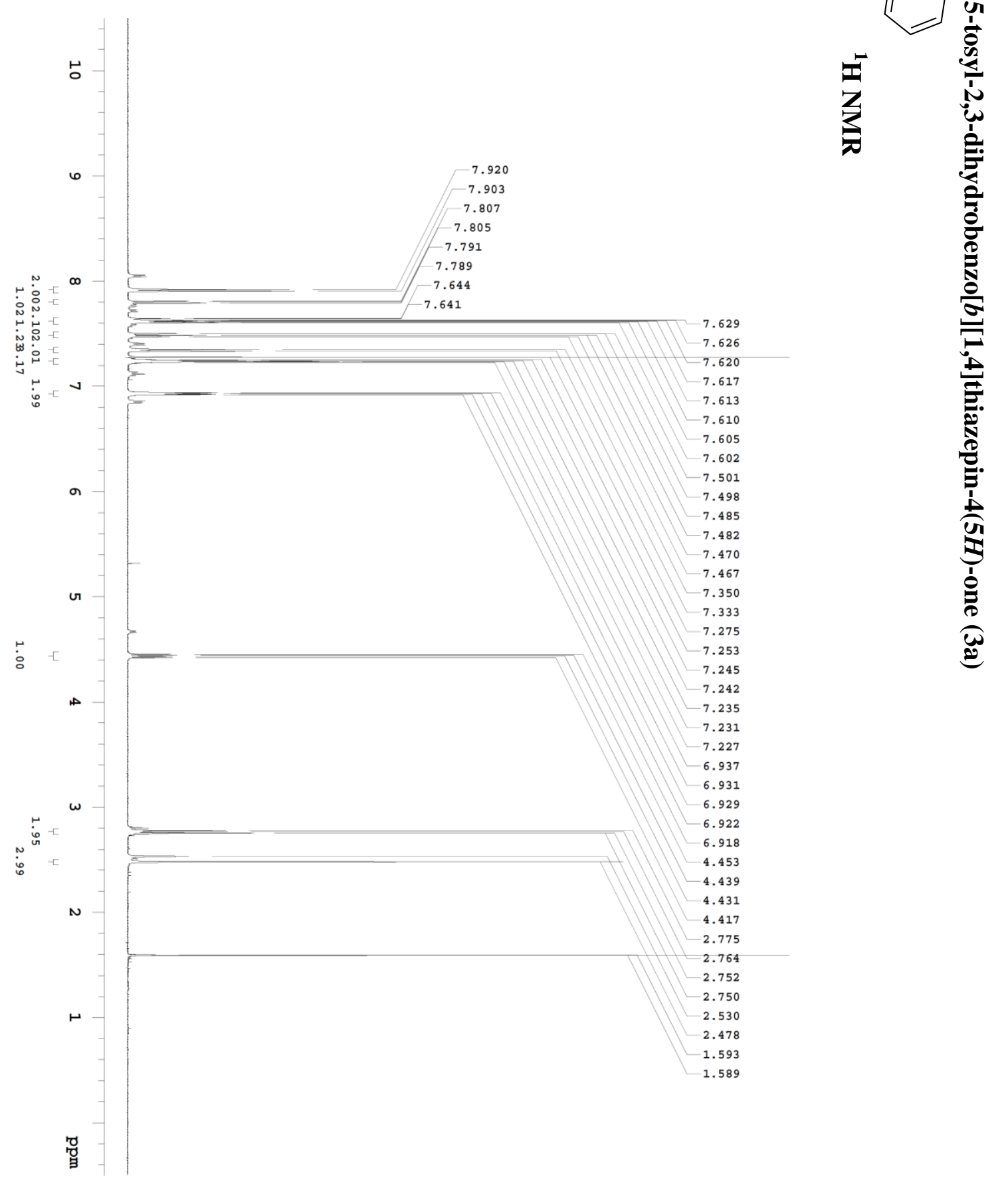



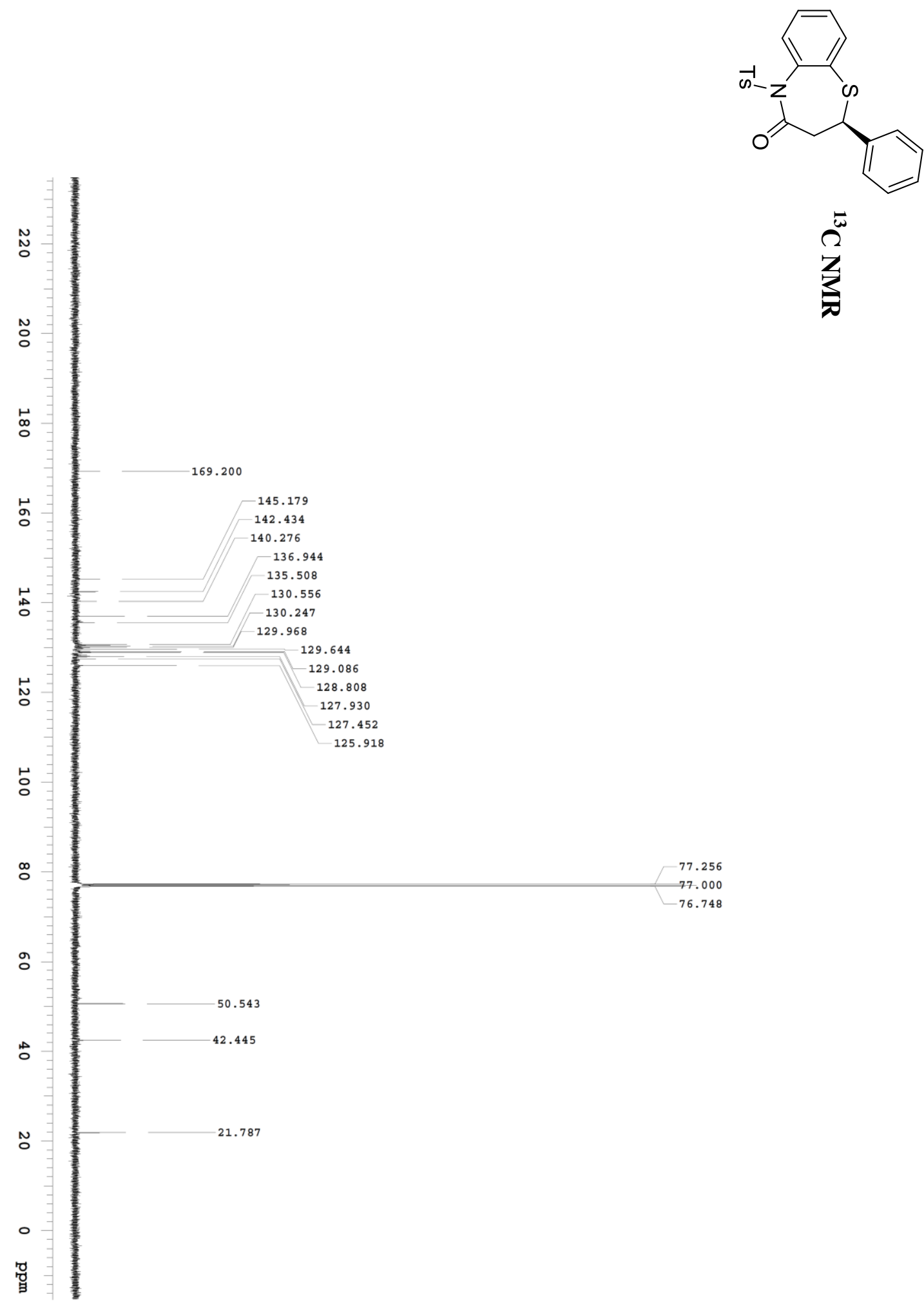

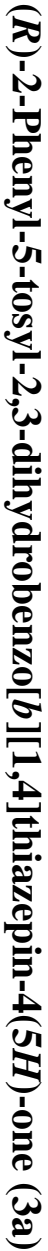

$\overbrace{}^{\bar{\omega}}$

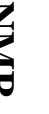



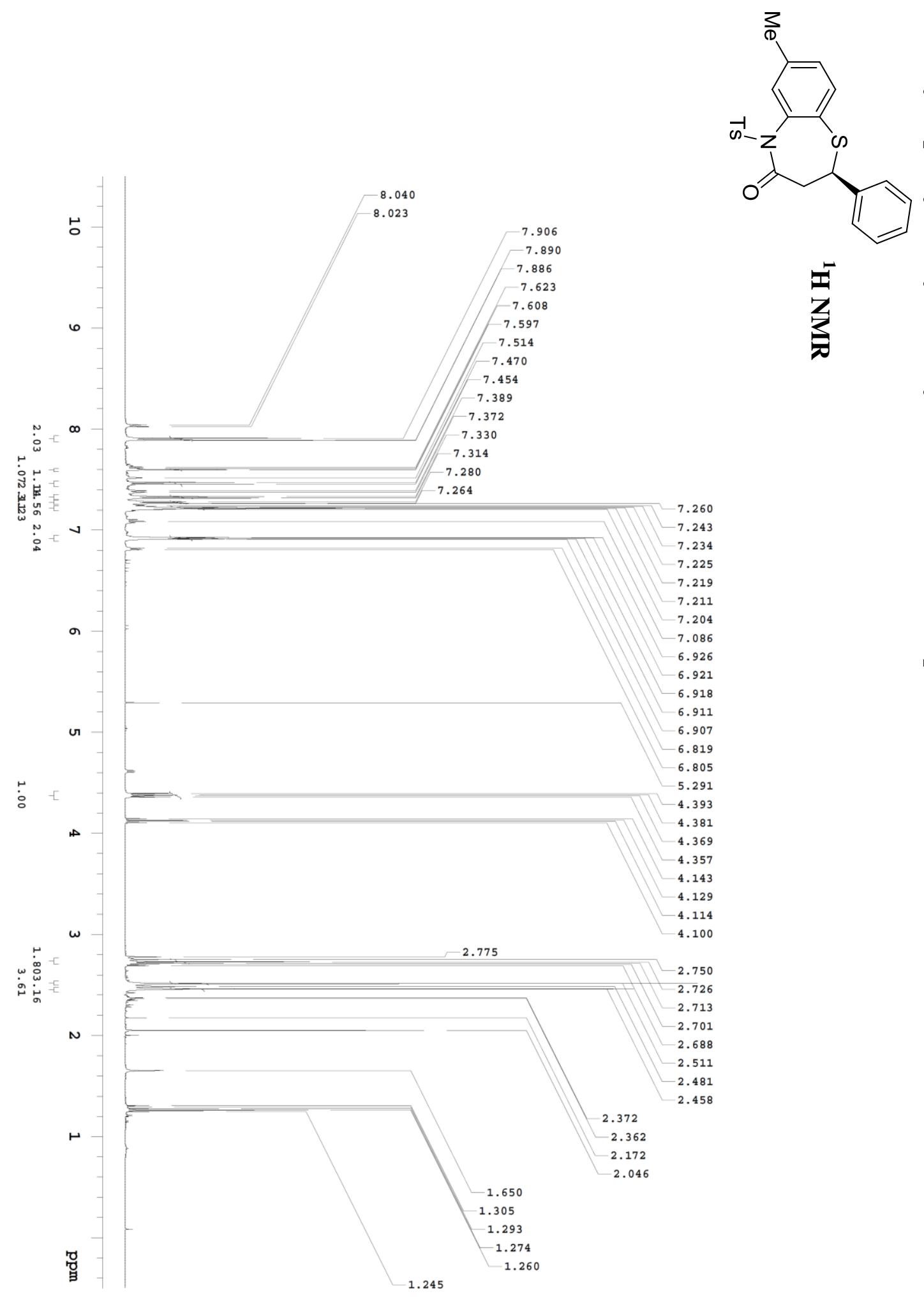

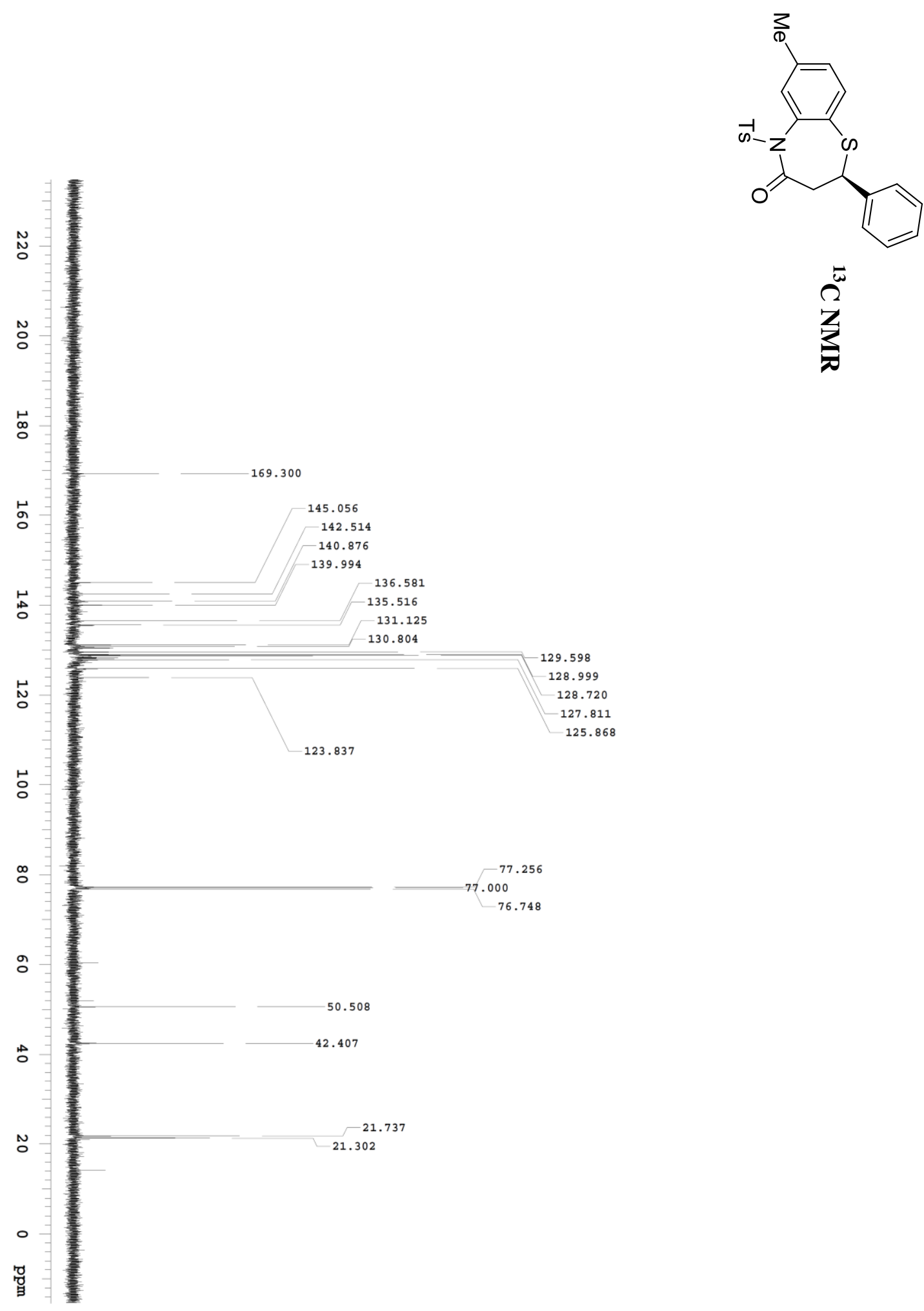

草

$\overbrace{}^{2}$

1ัن

 

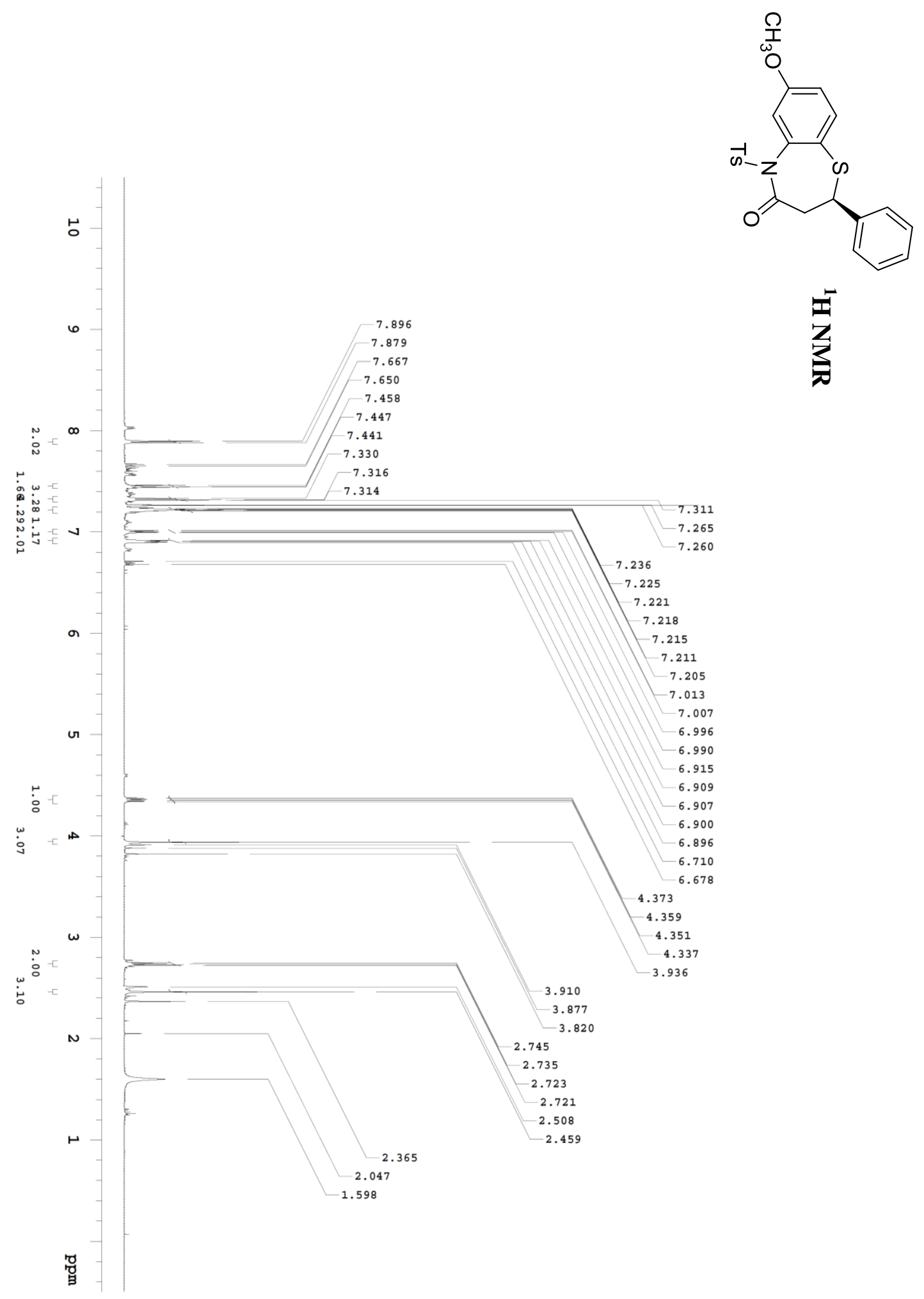

吾

它 

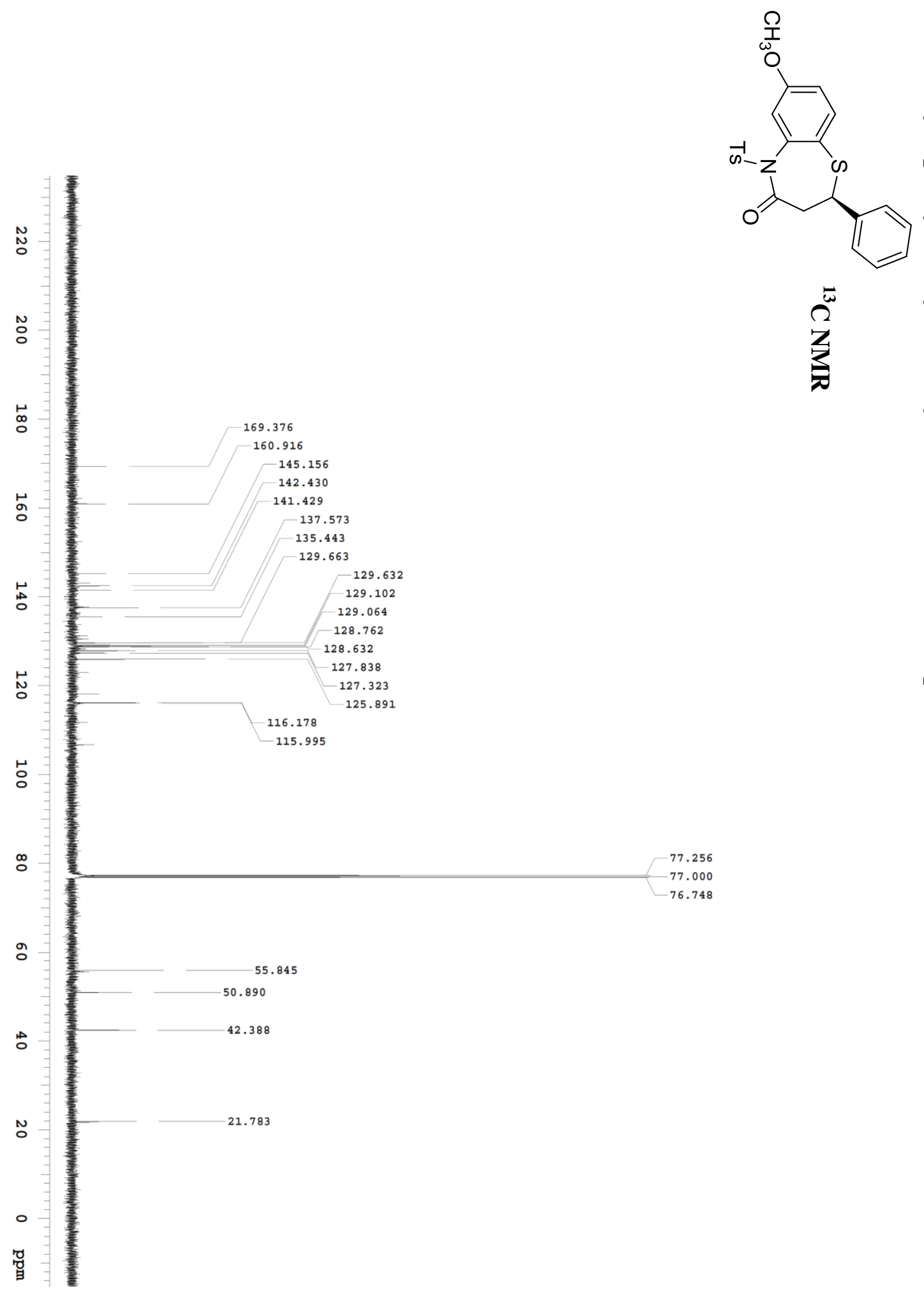

3.'

$\stackrel{2}{2}^{2}$

لِّ

: 

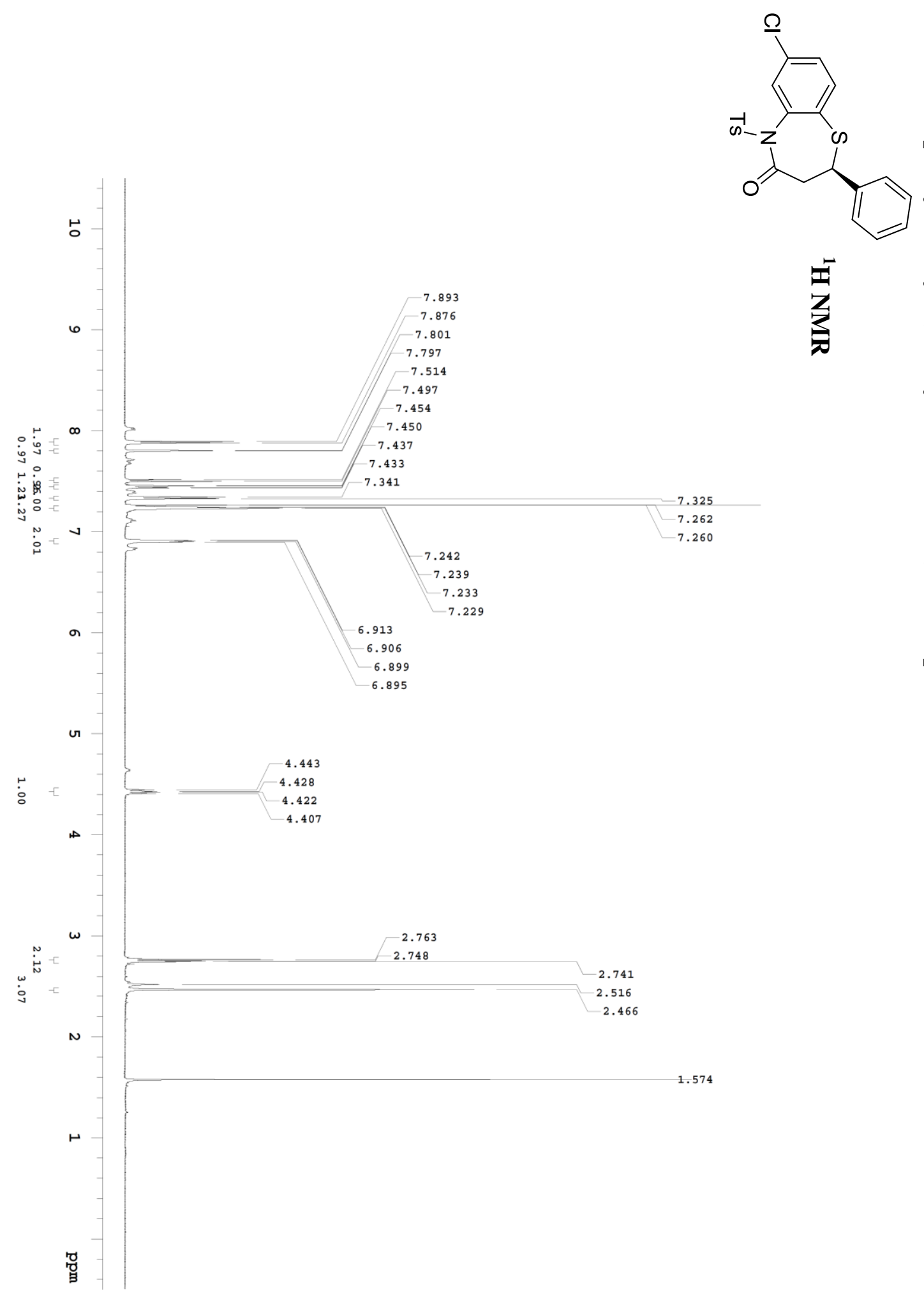

อُ

量

它

.

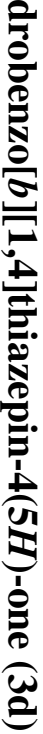



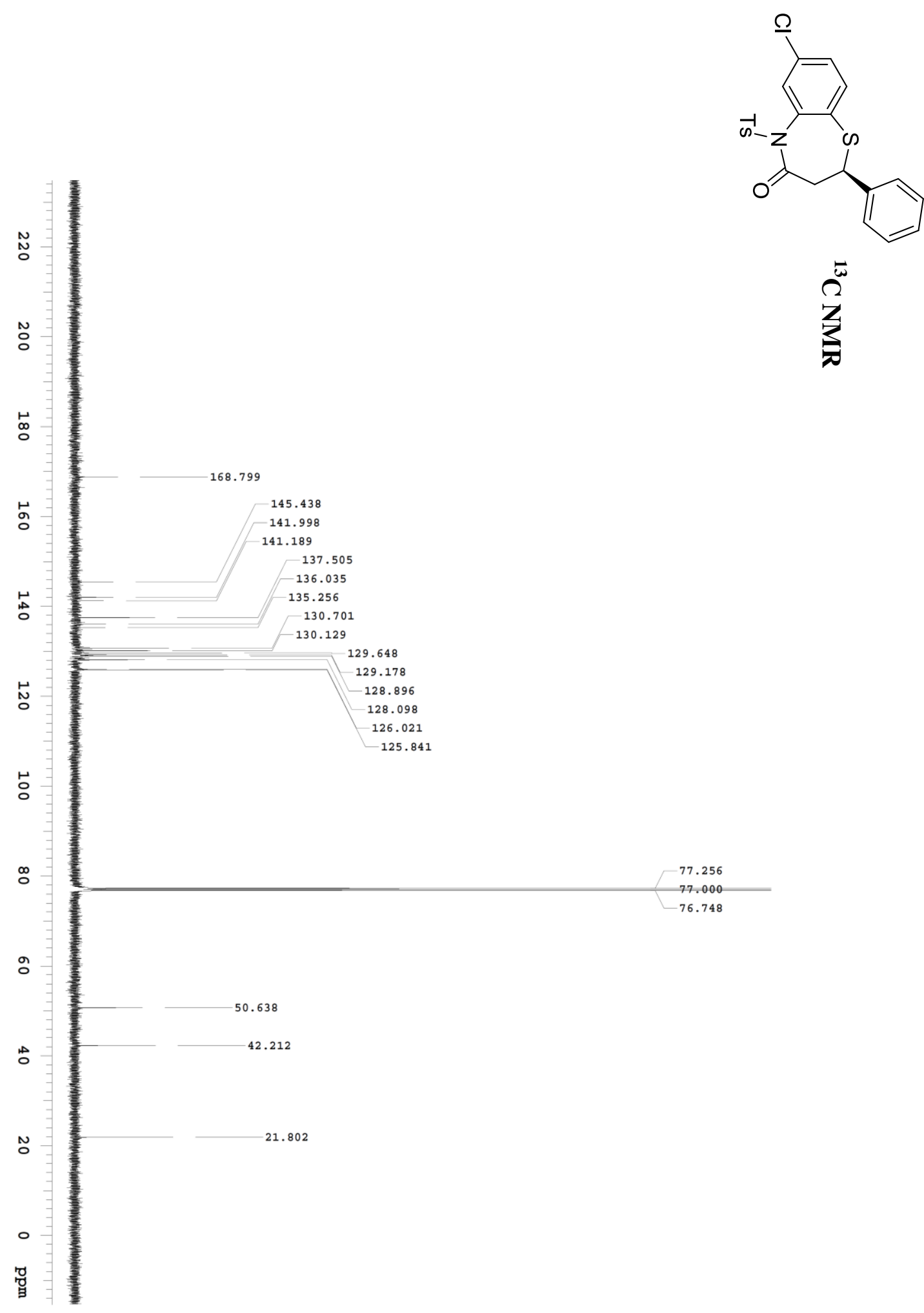

Зُ

$\overbrace{}^{2}$ 

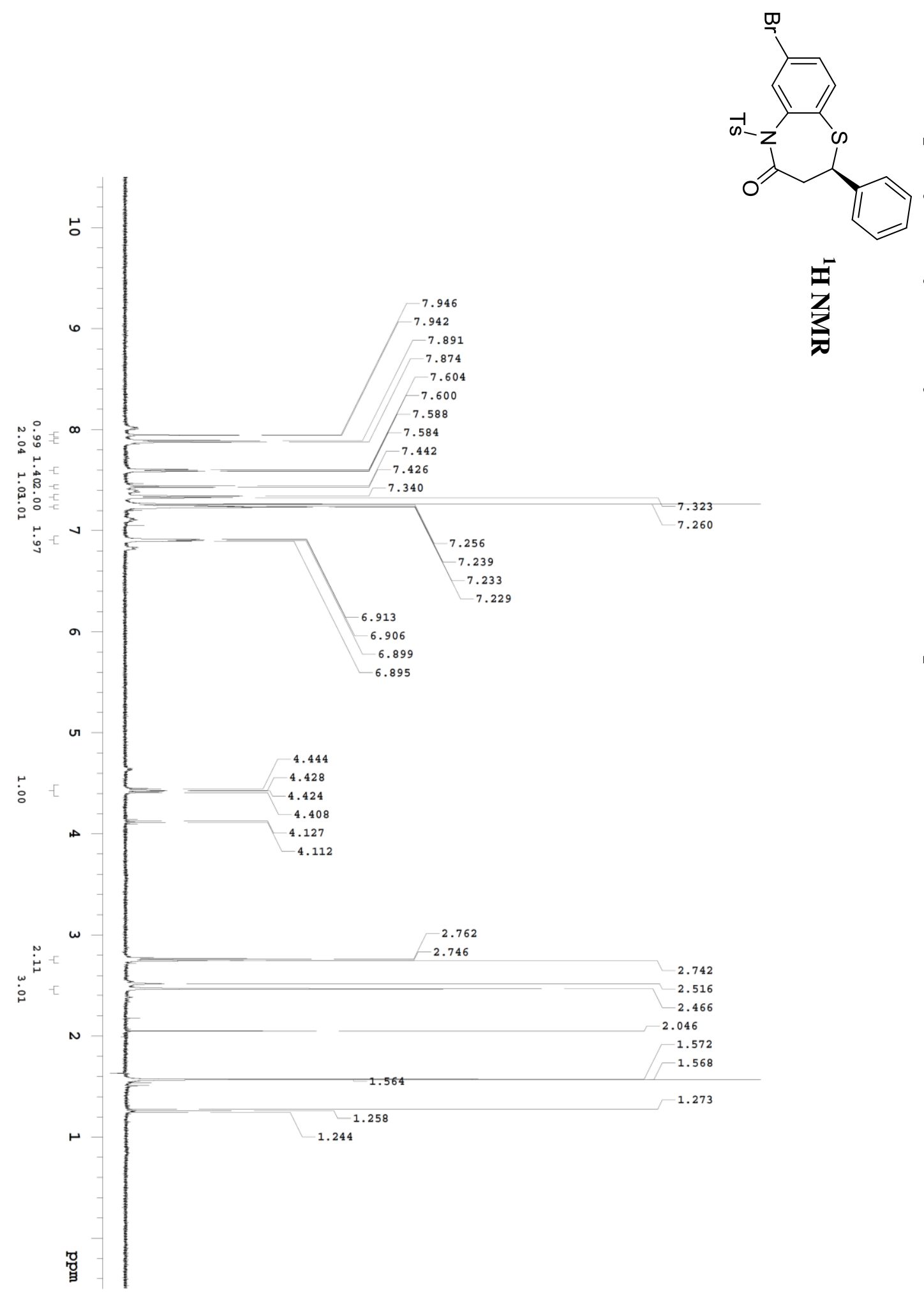

'0

叓 

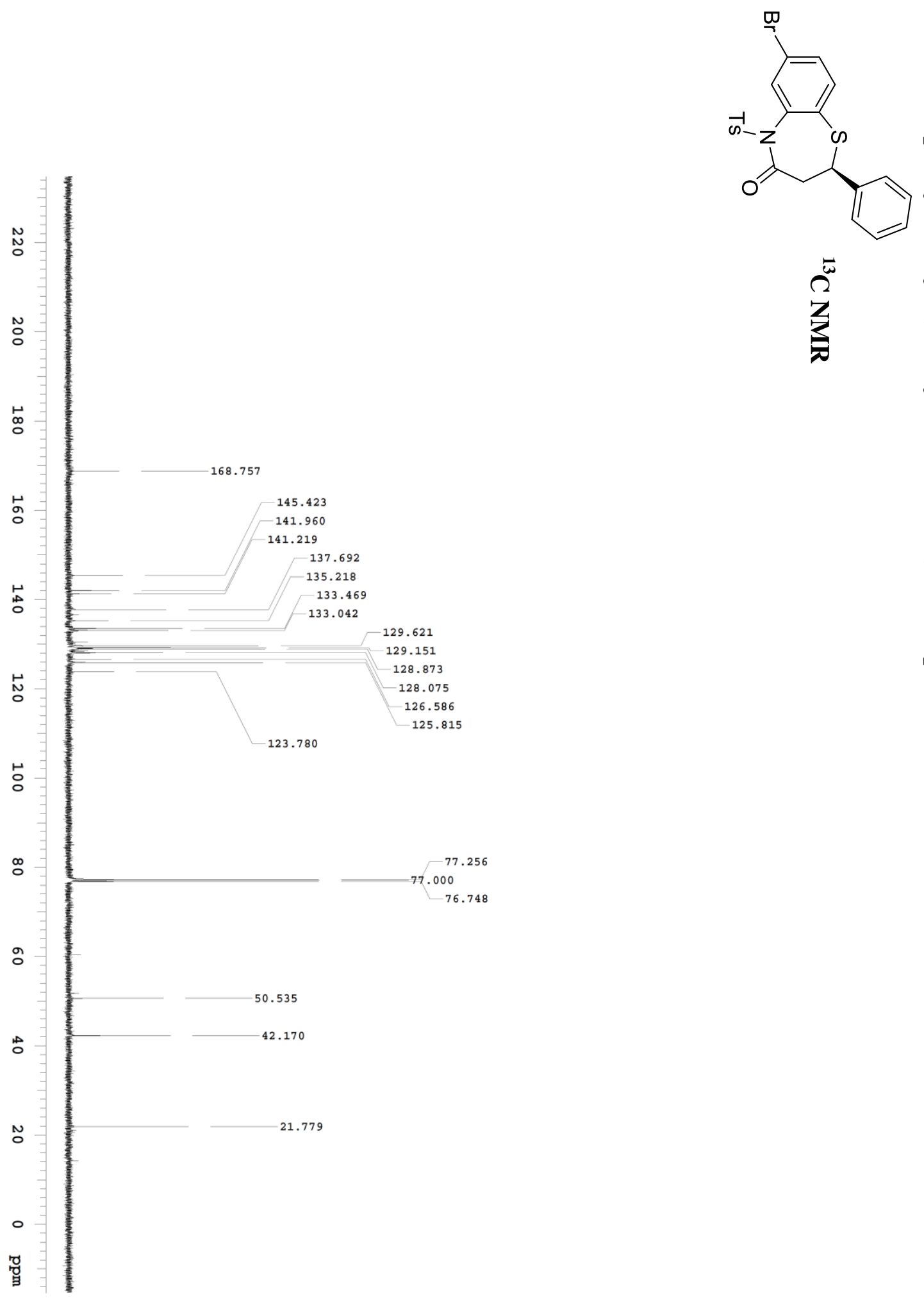

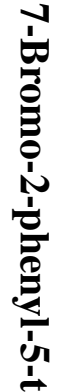

$\overbrace{}^{\bar{\omega}}$

它

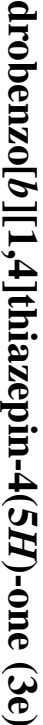



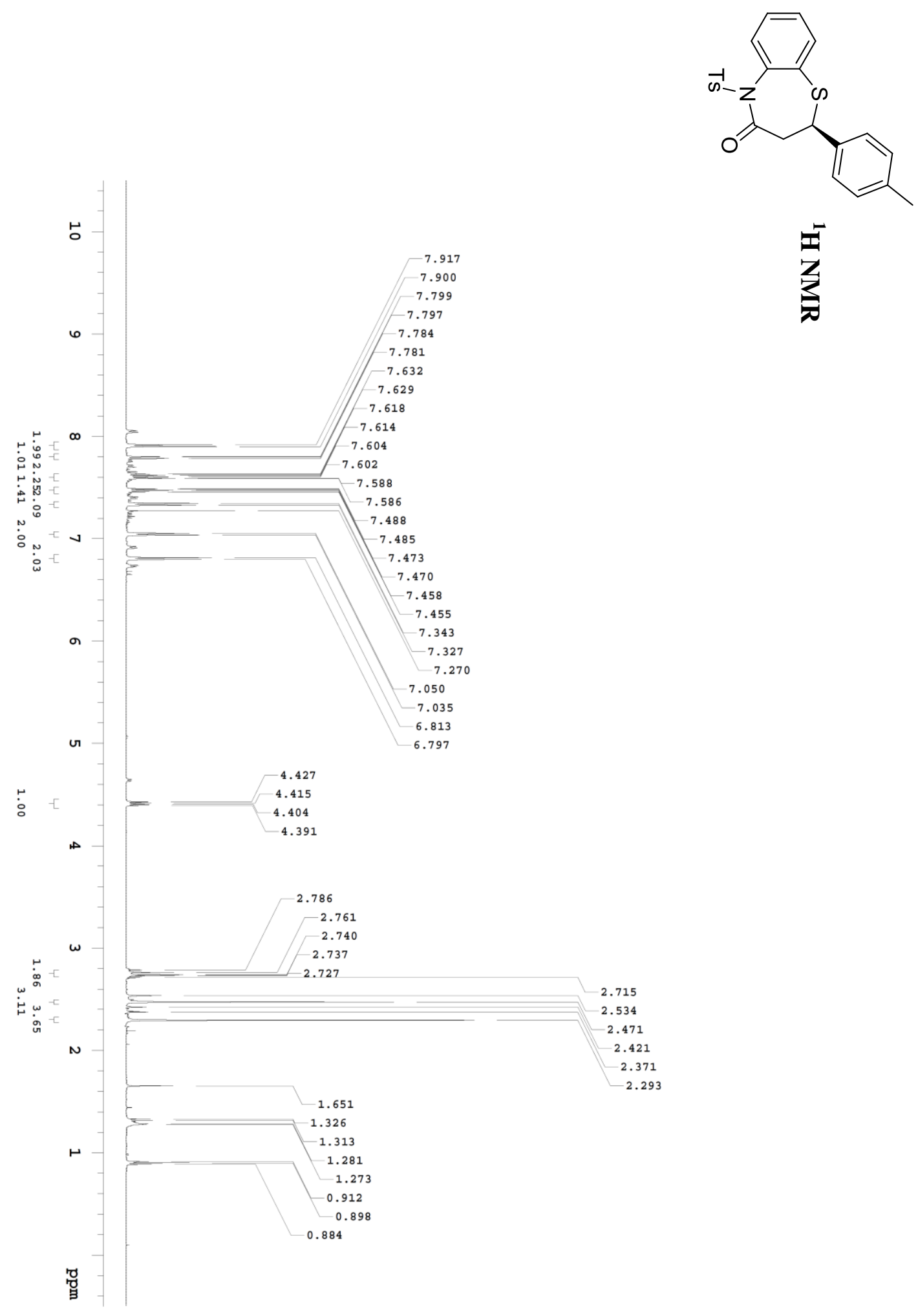

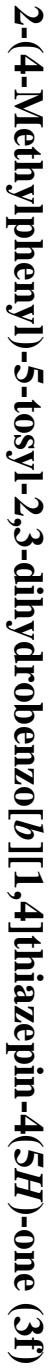



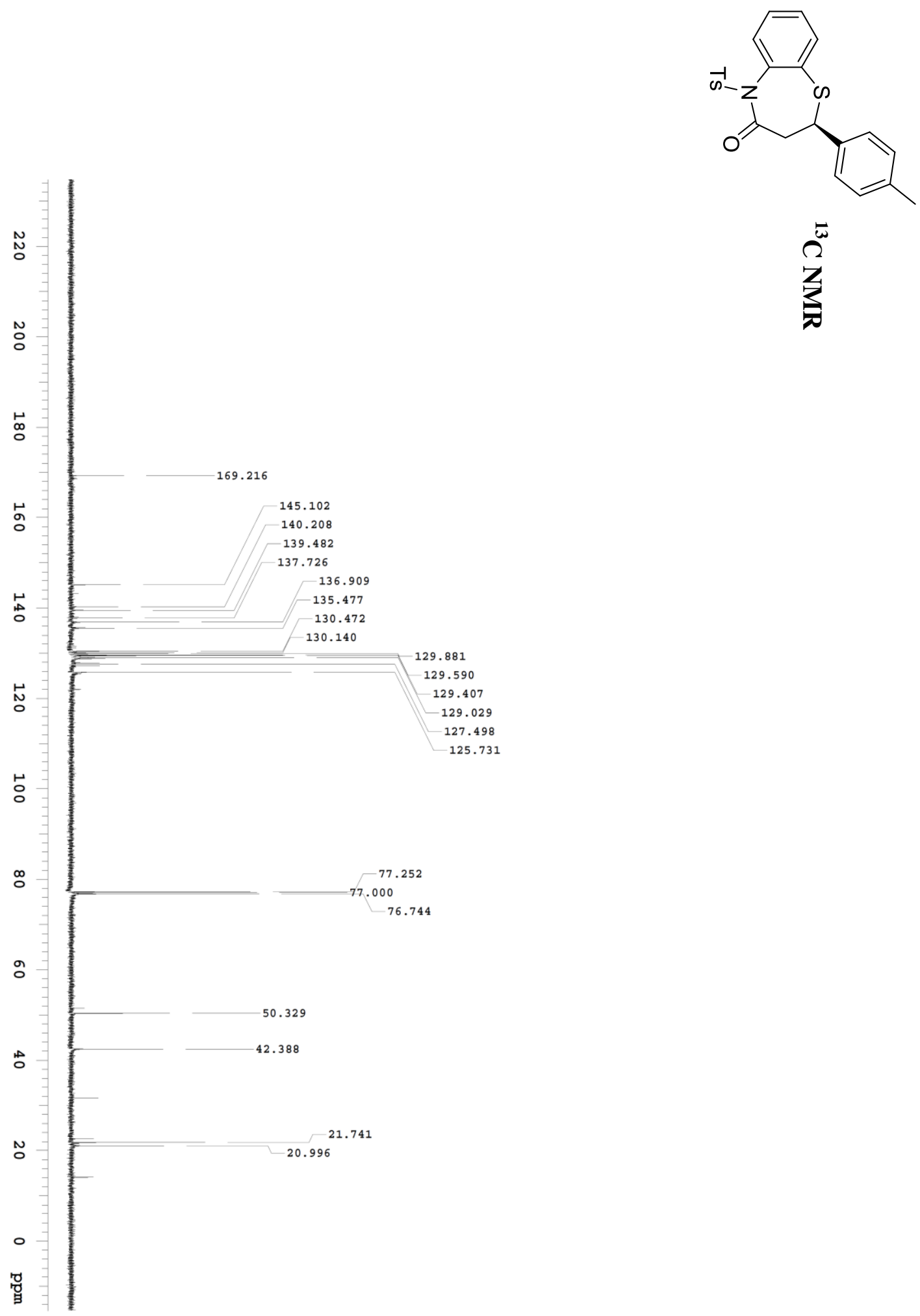

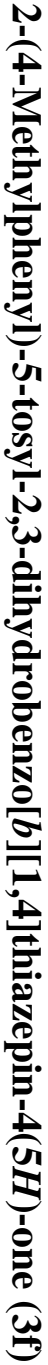




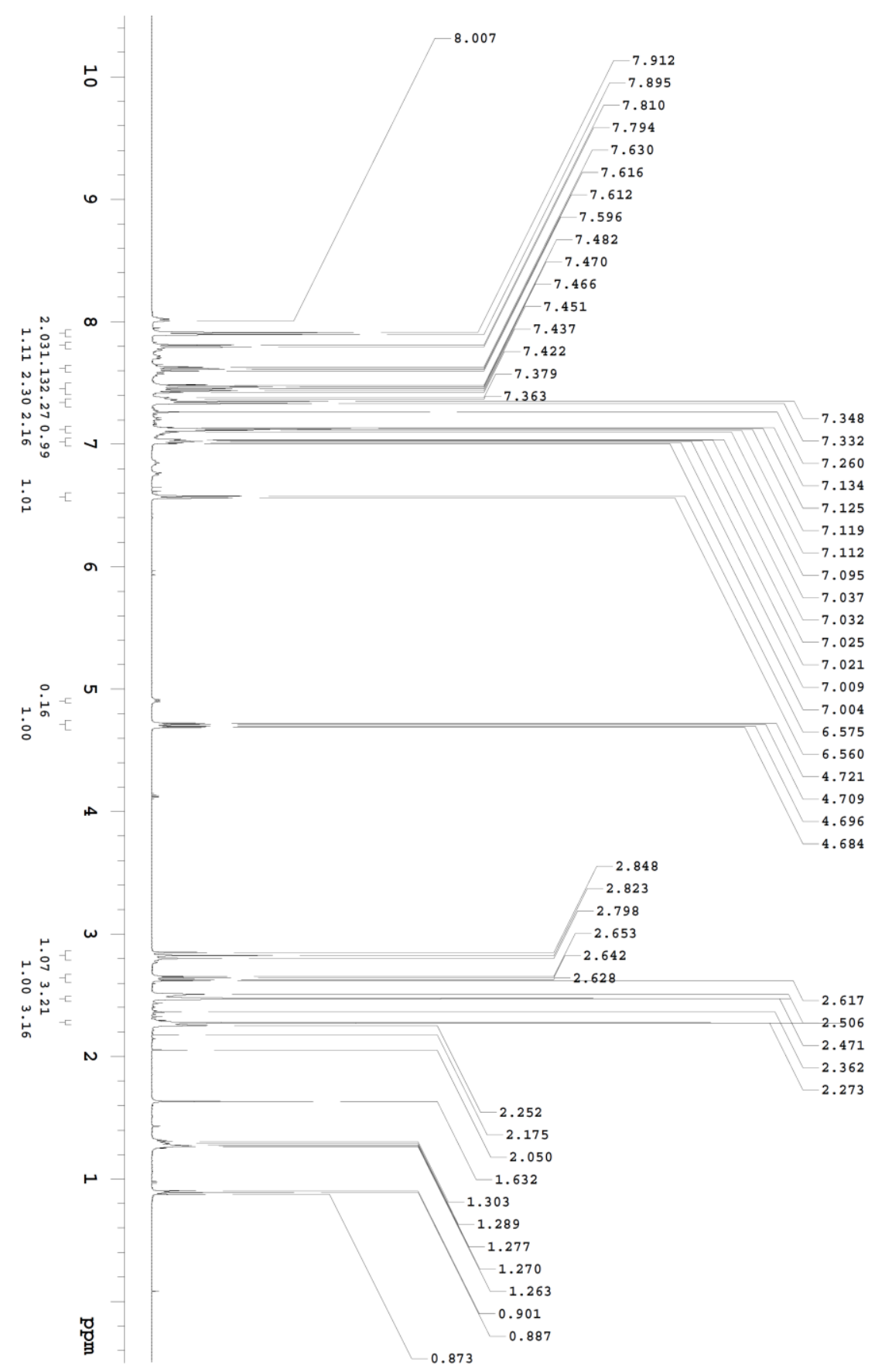



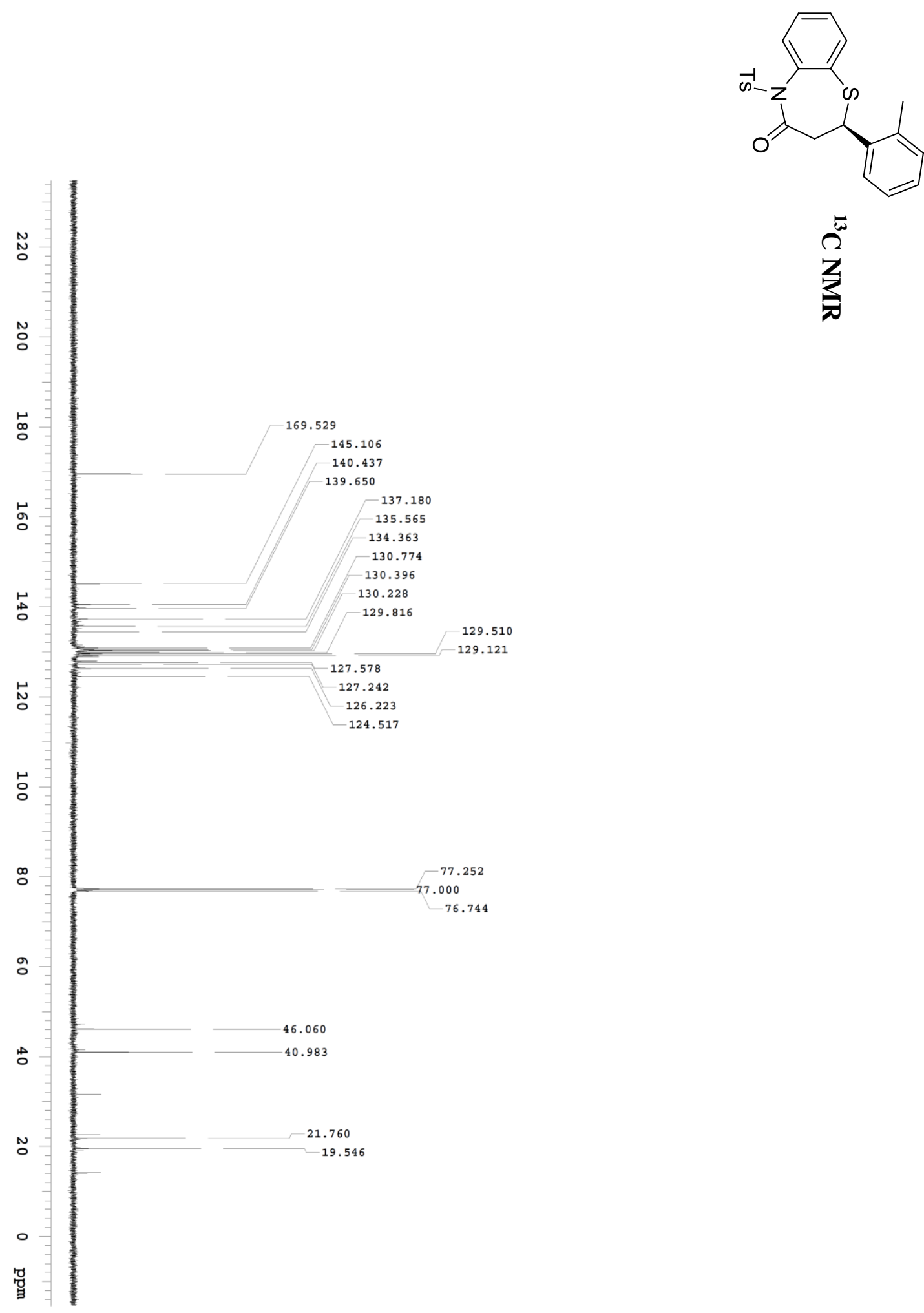

$\stackrel{2}{2}^{2}$

ט. 


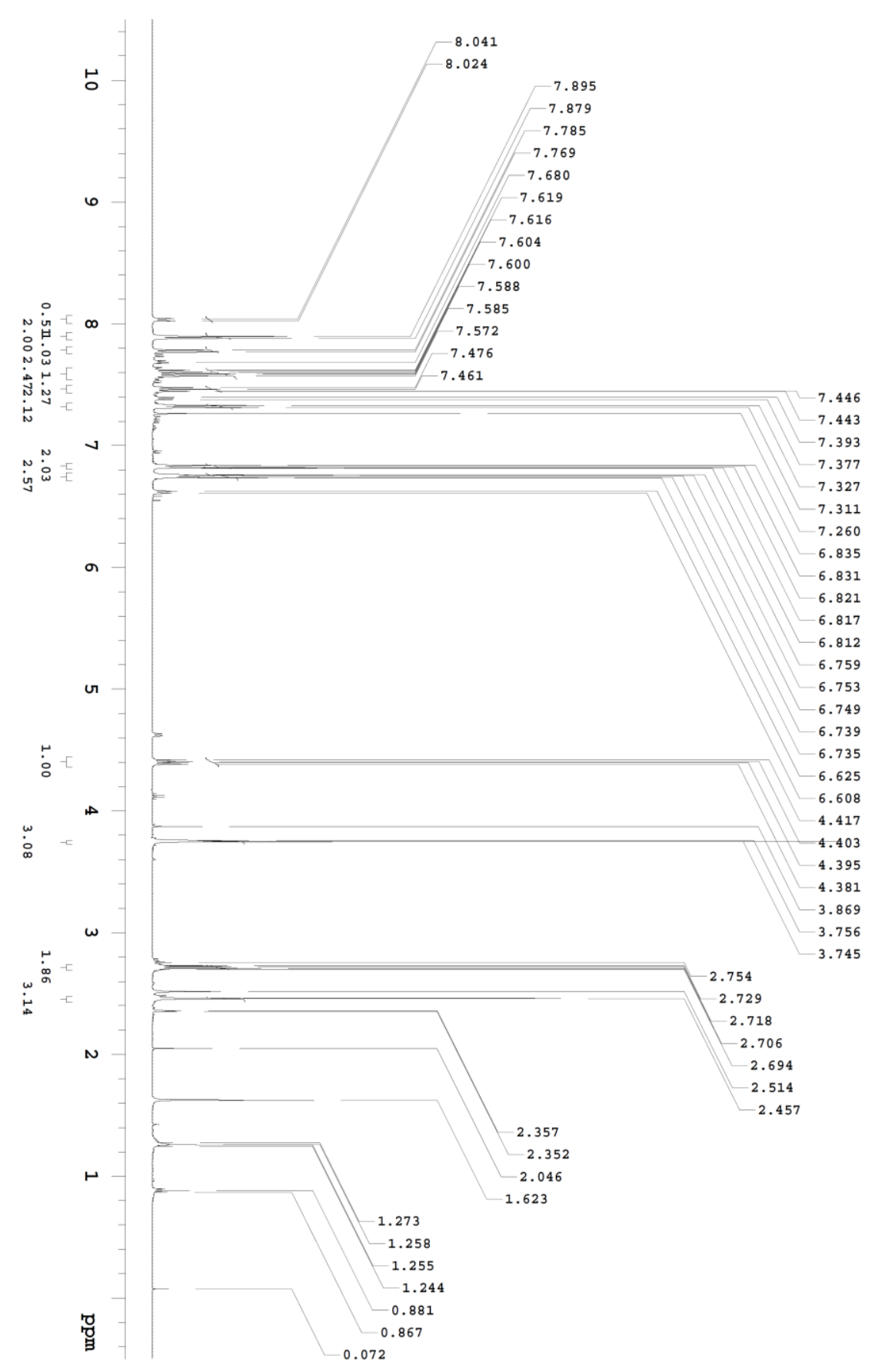



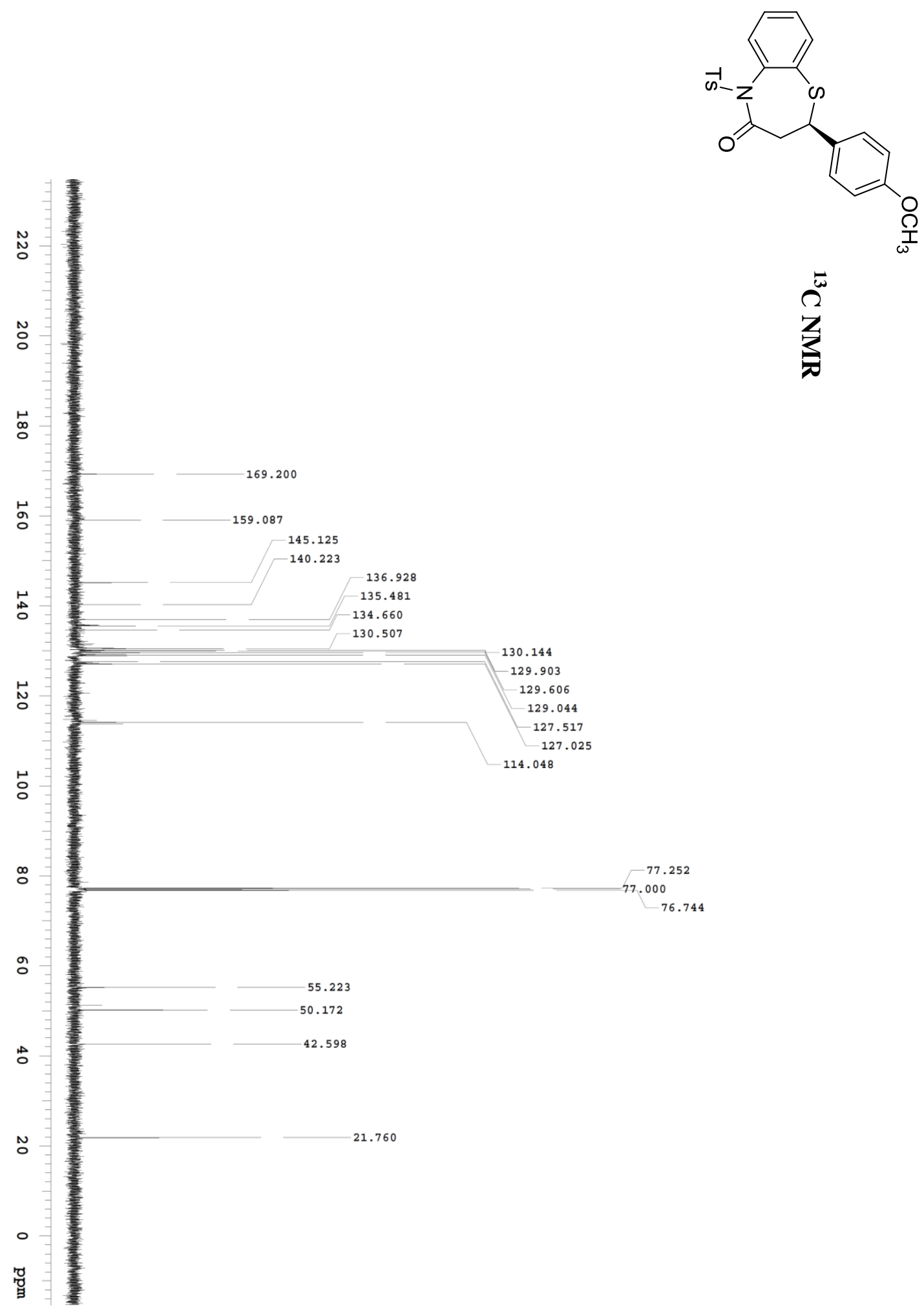

مُ

$\overbrace{}^{2}$

告 

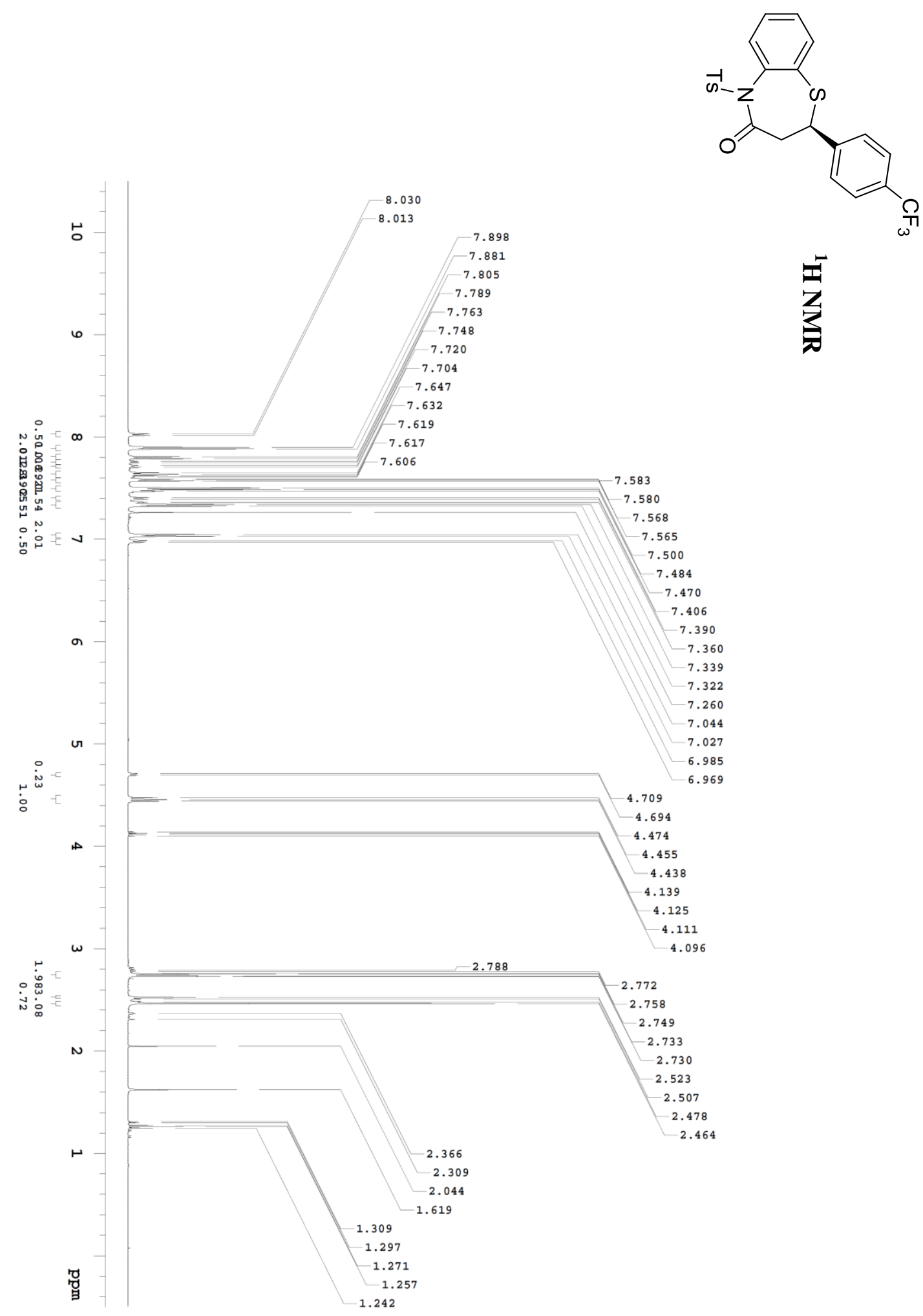

U. 

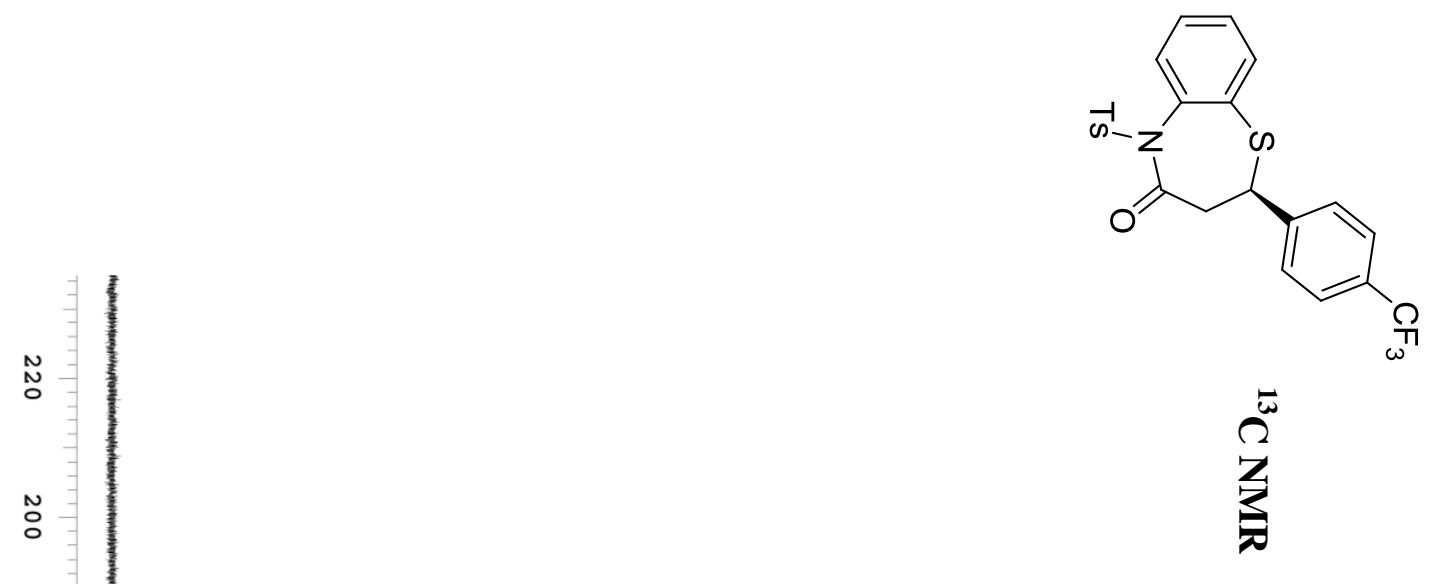

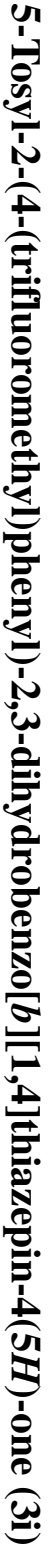
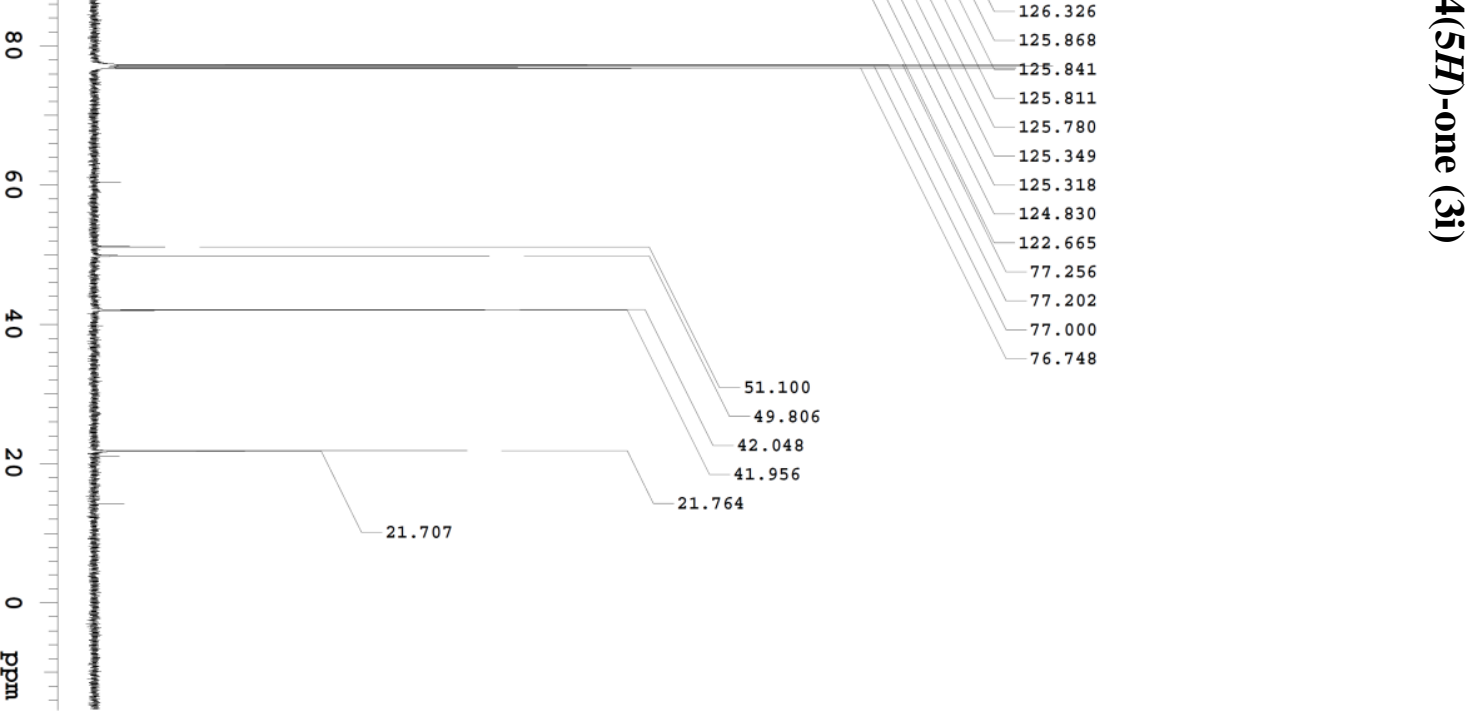

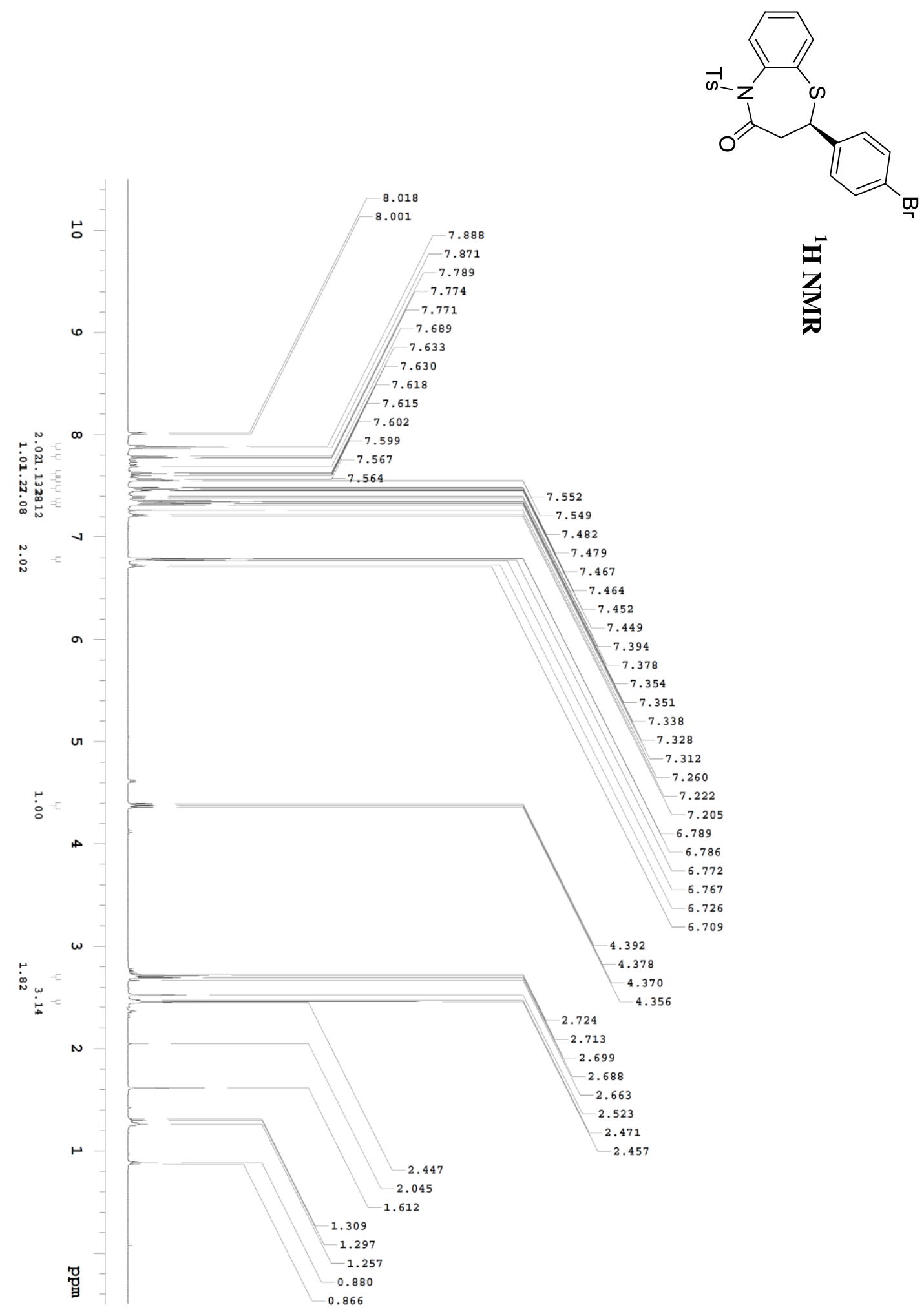

艺

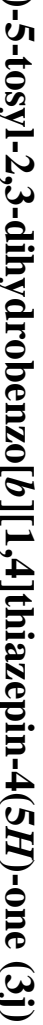



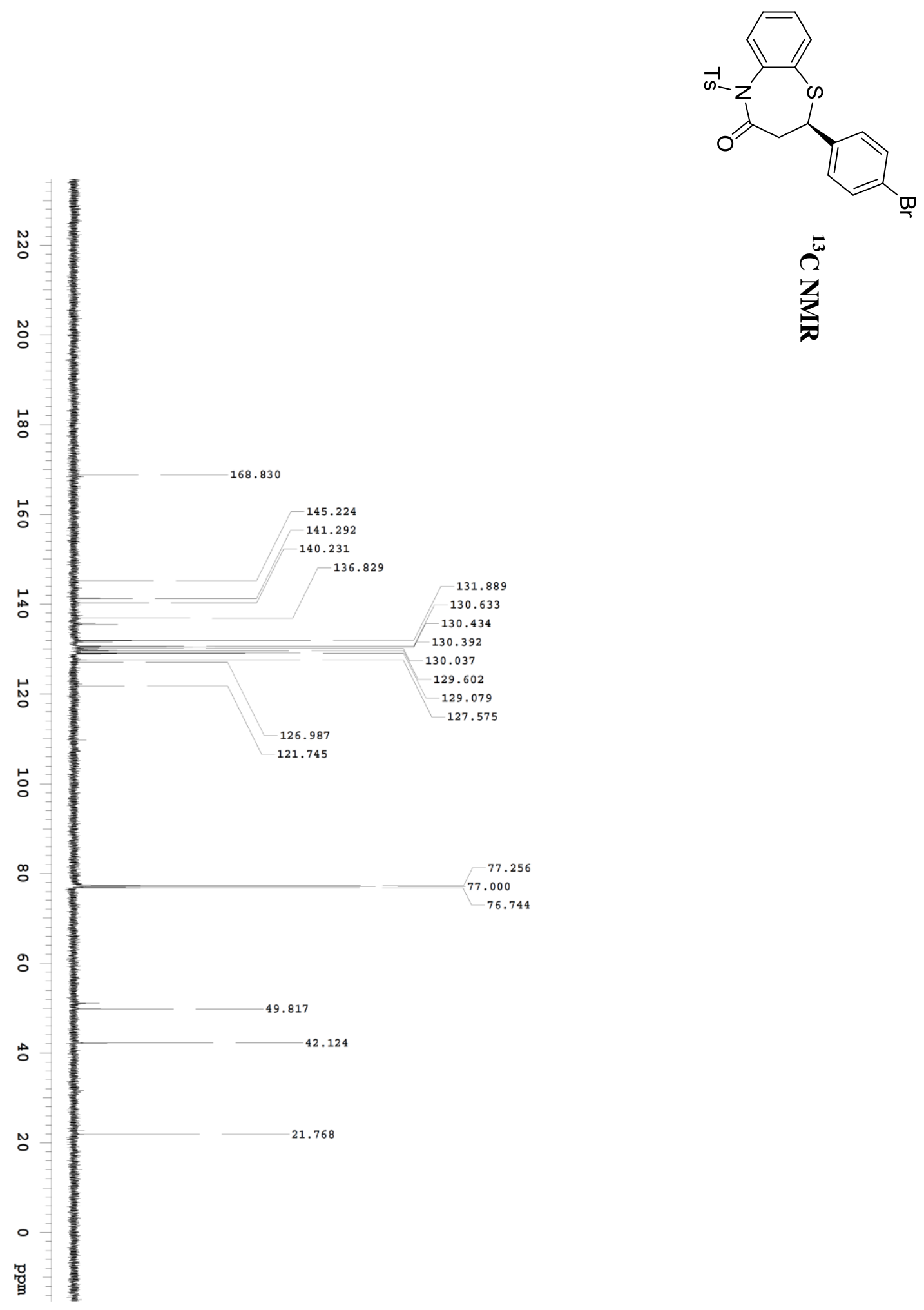

草

$\overbrace{}^{2}$

لَ. 

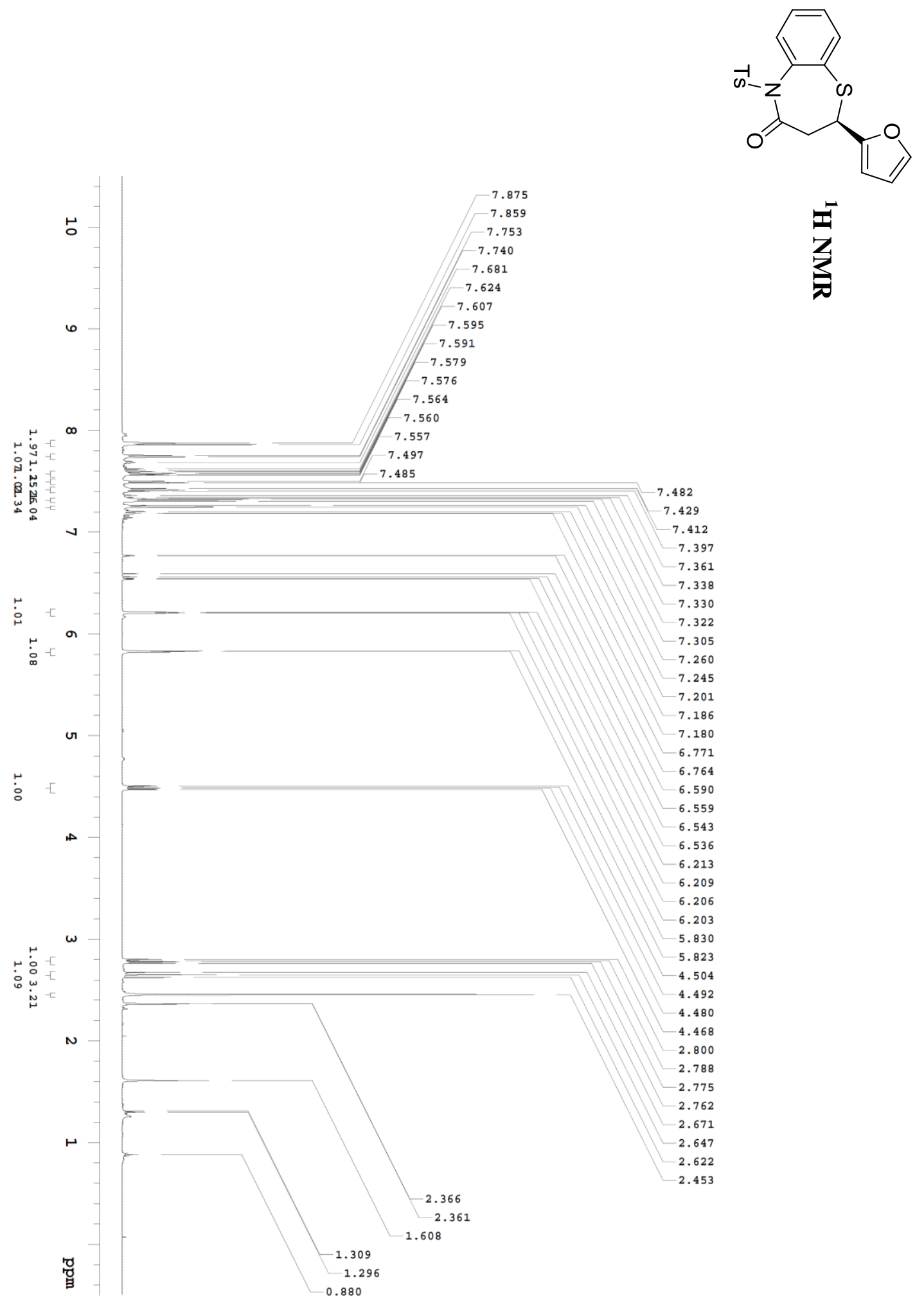

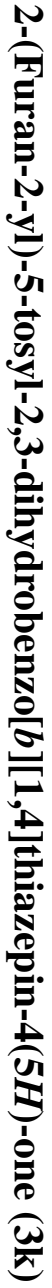



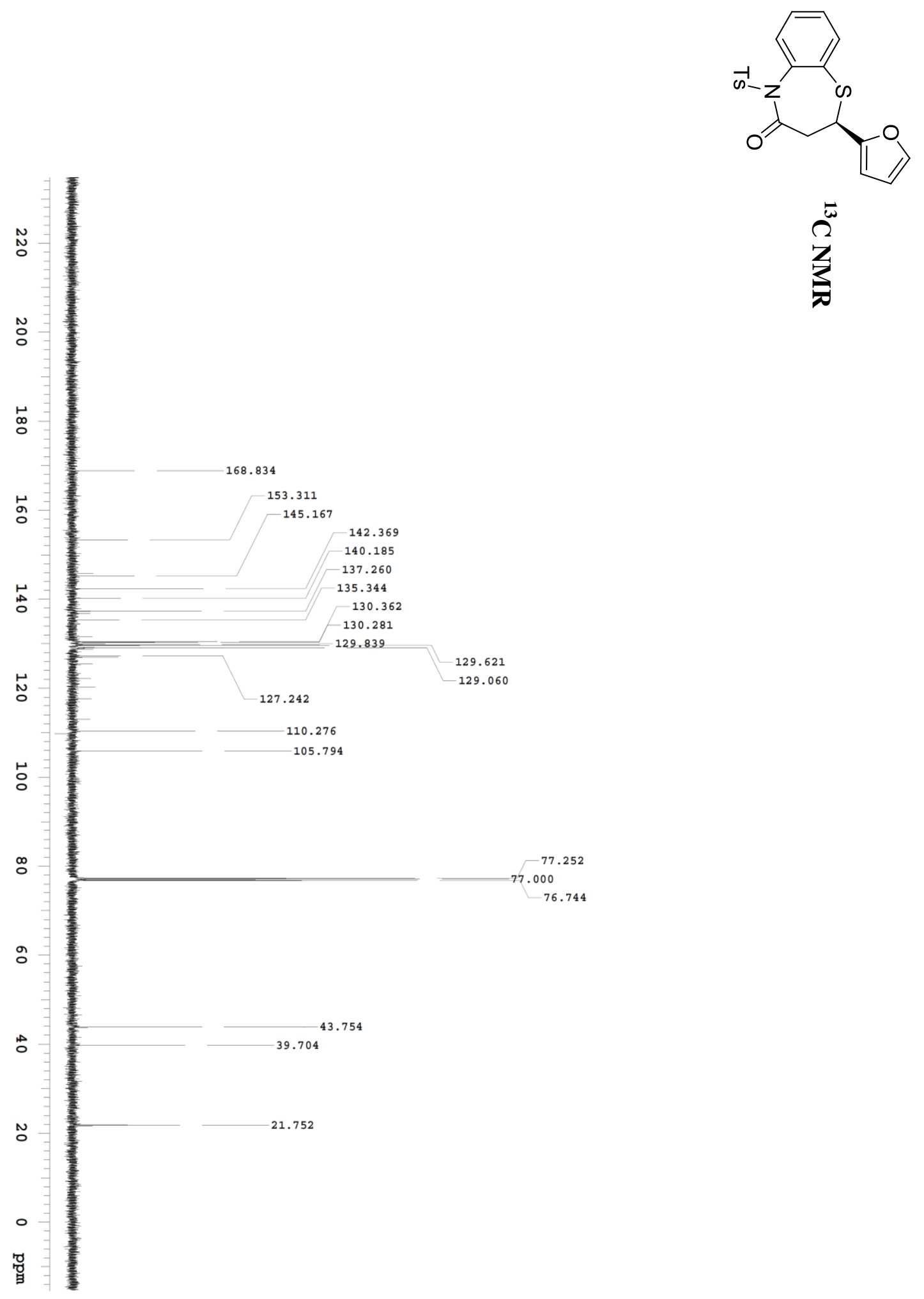



$\stackrel{2}{z}^{2}$ 


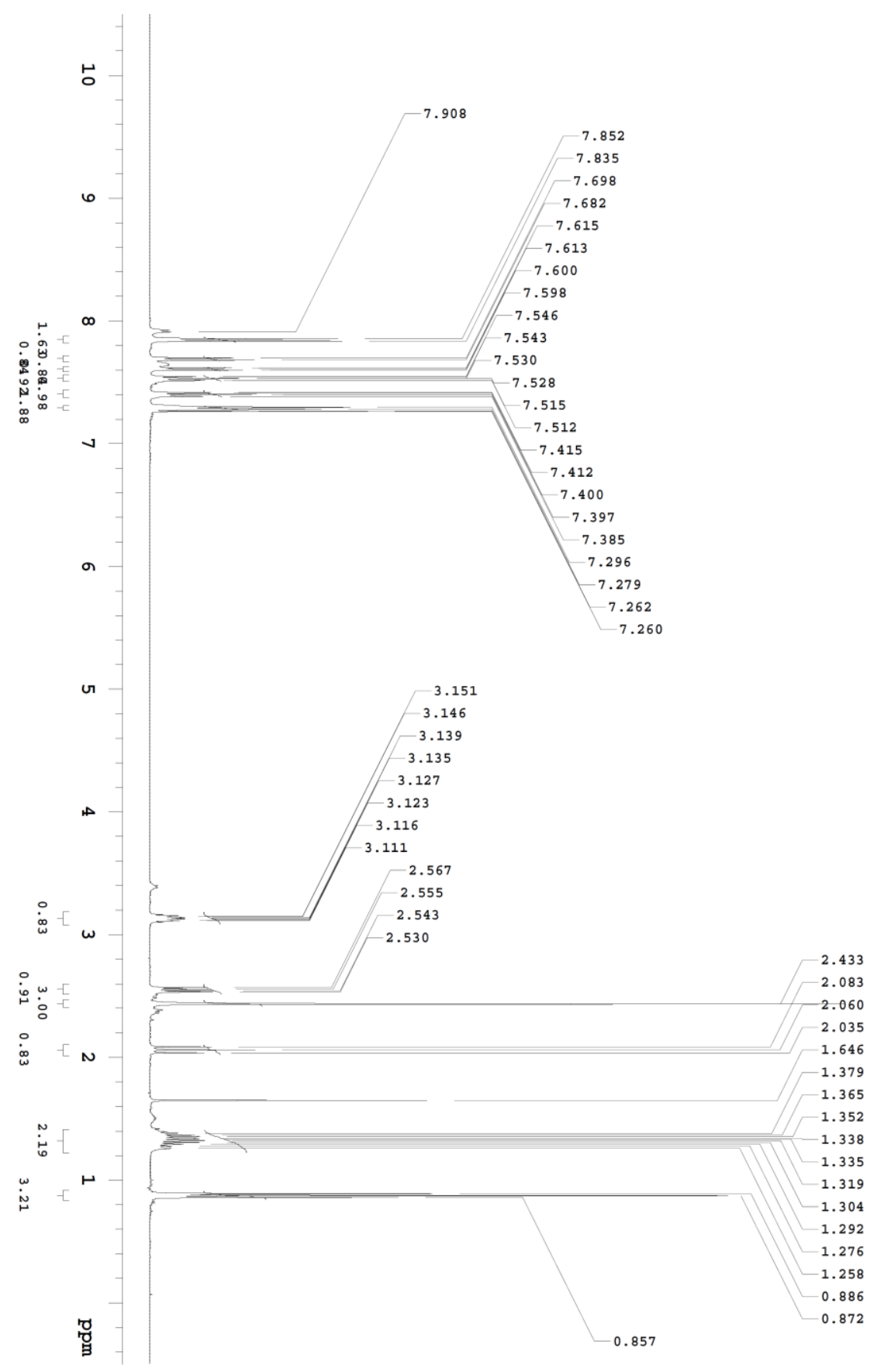

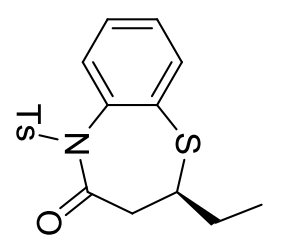

吾 

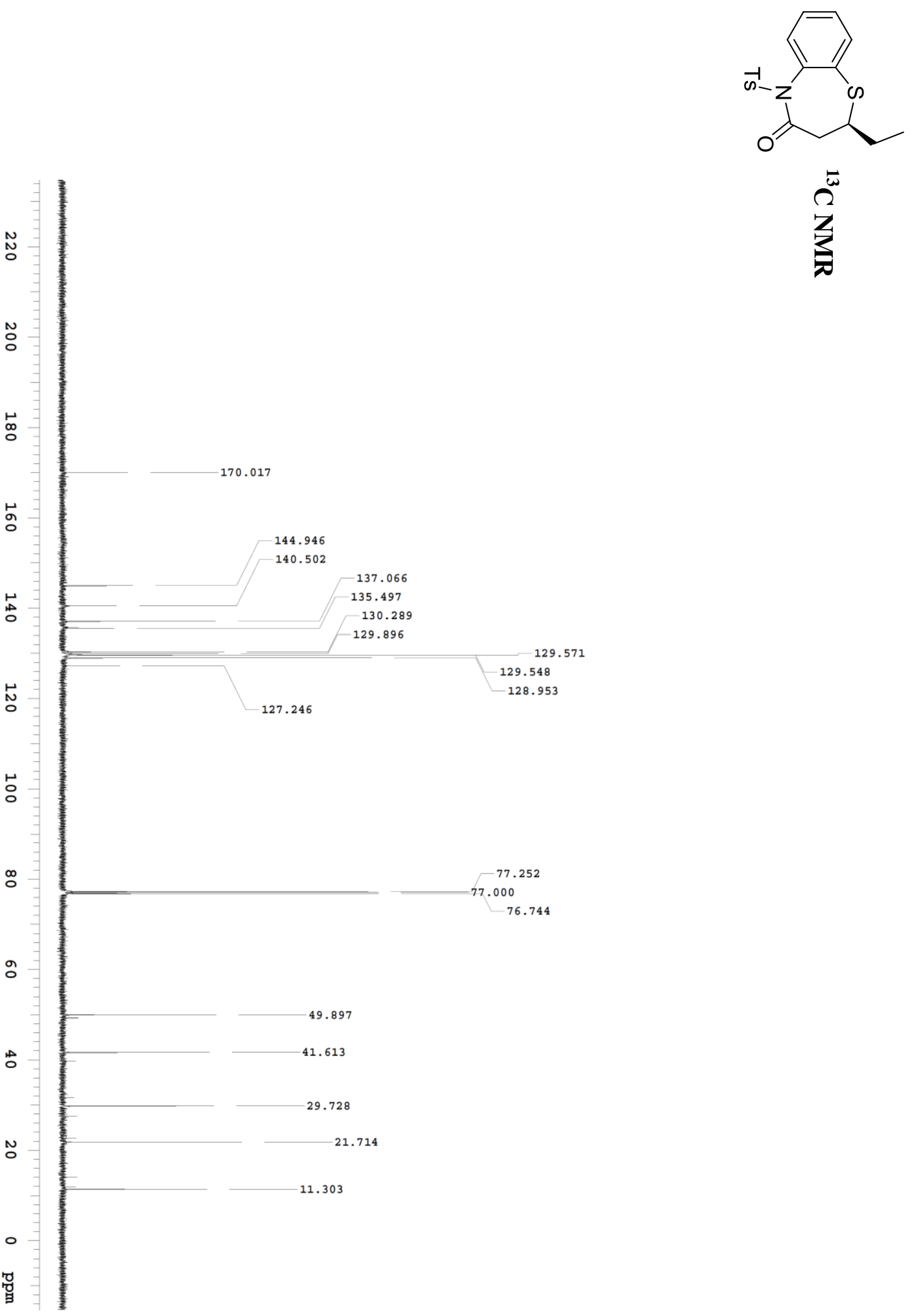

$\overbrace{}^{2}$

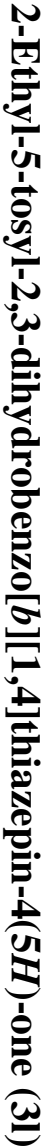




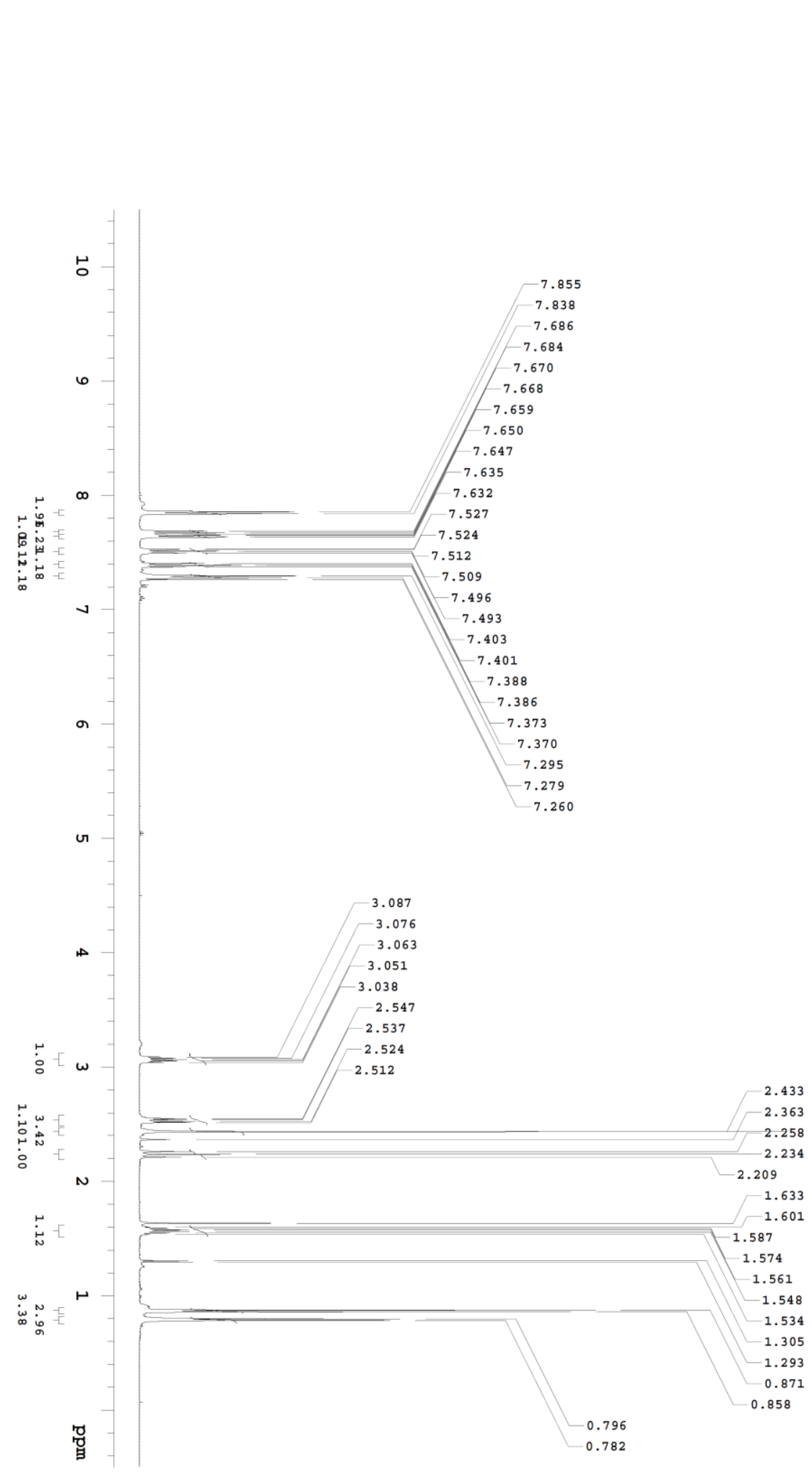



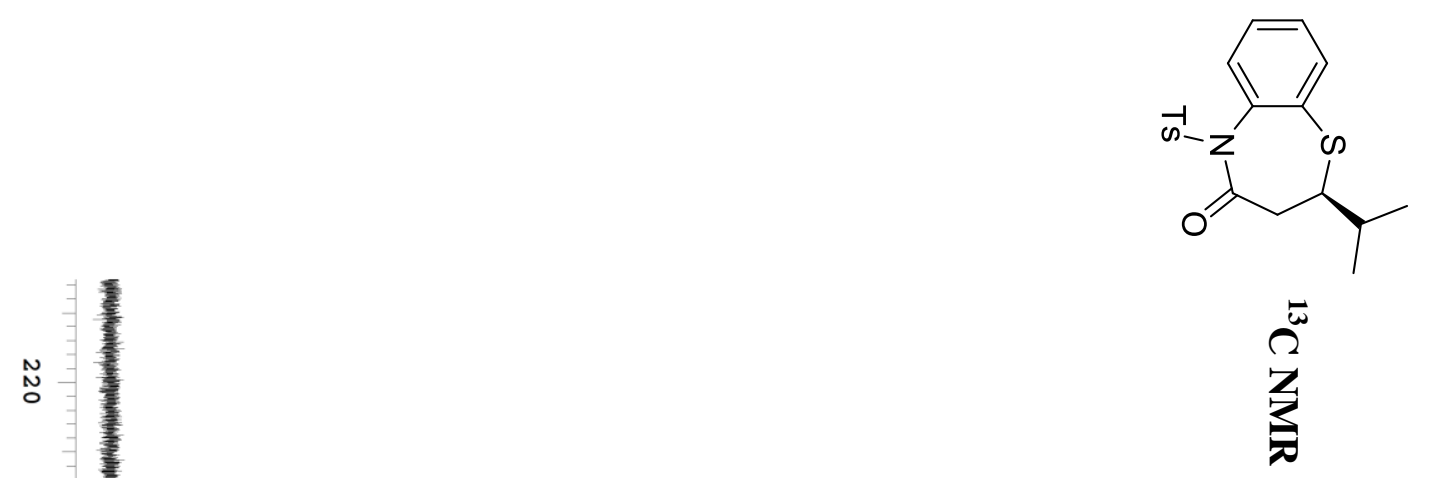

$\overbrace{}^{\sqrt[\omega]{0}}$

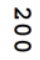

$\stackrel{\leftrightarrow}{\circ}$

म.

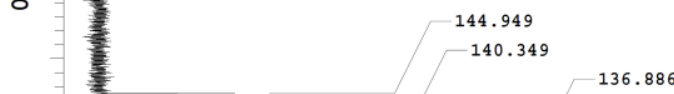
-135.516
-130.14

$-129.648$ $-129.541$

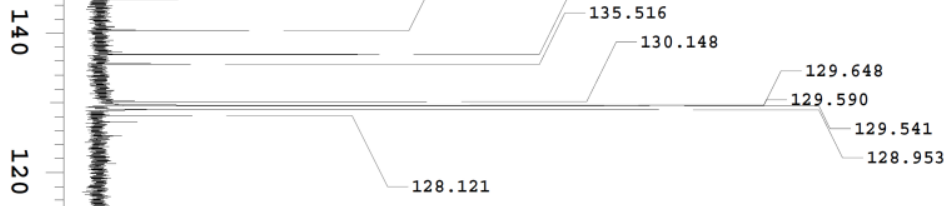

官

象

Ð્త

$\stackrel{\infty}{\circ}$

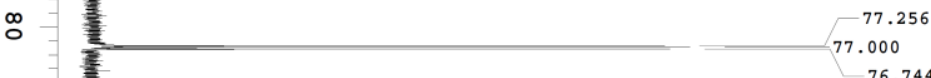

응

b

N

$-21.722$

19.332

目

76.74 

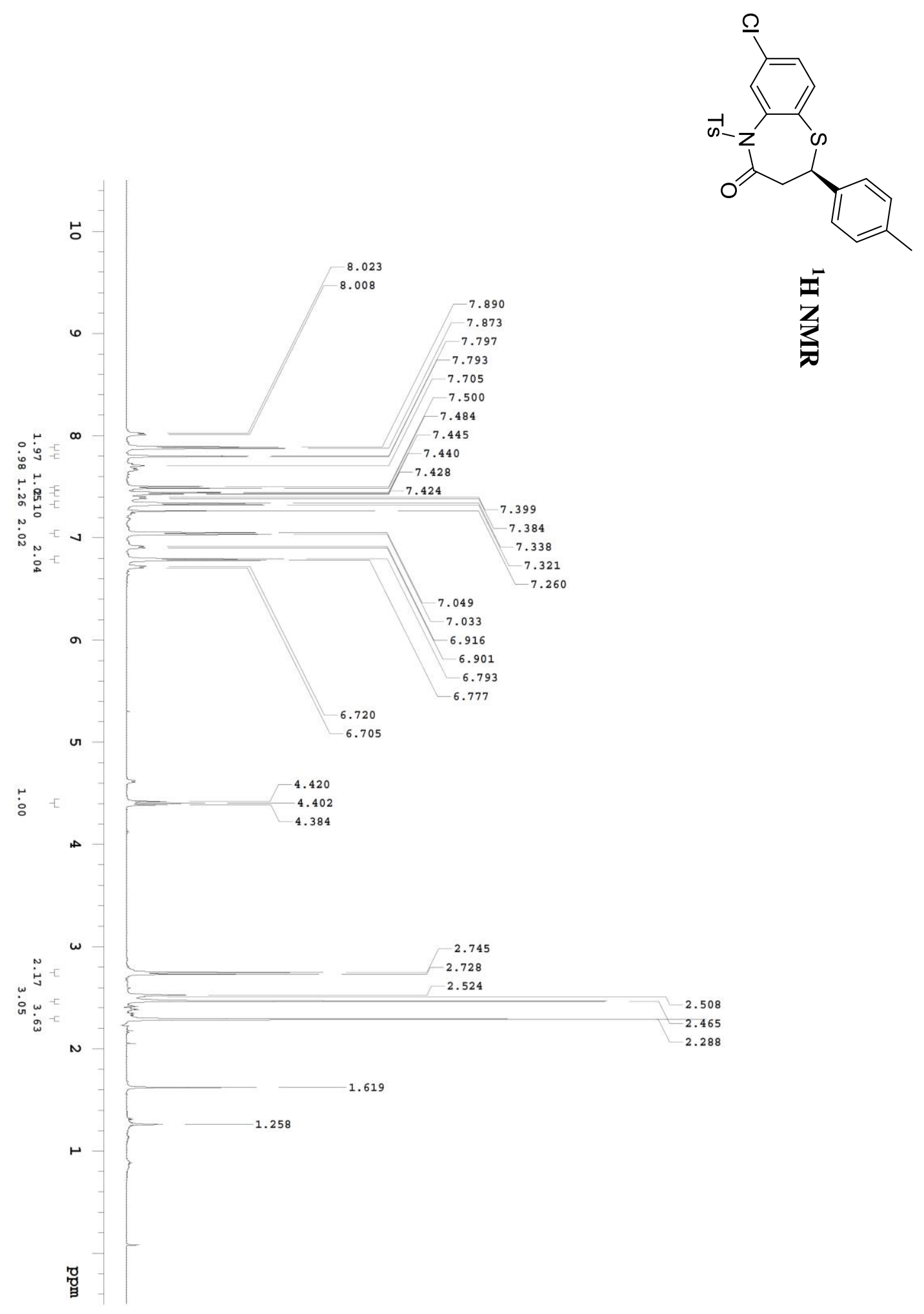

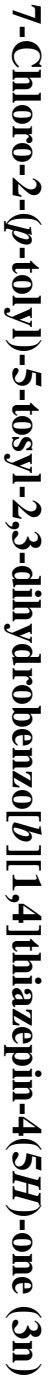



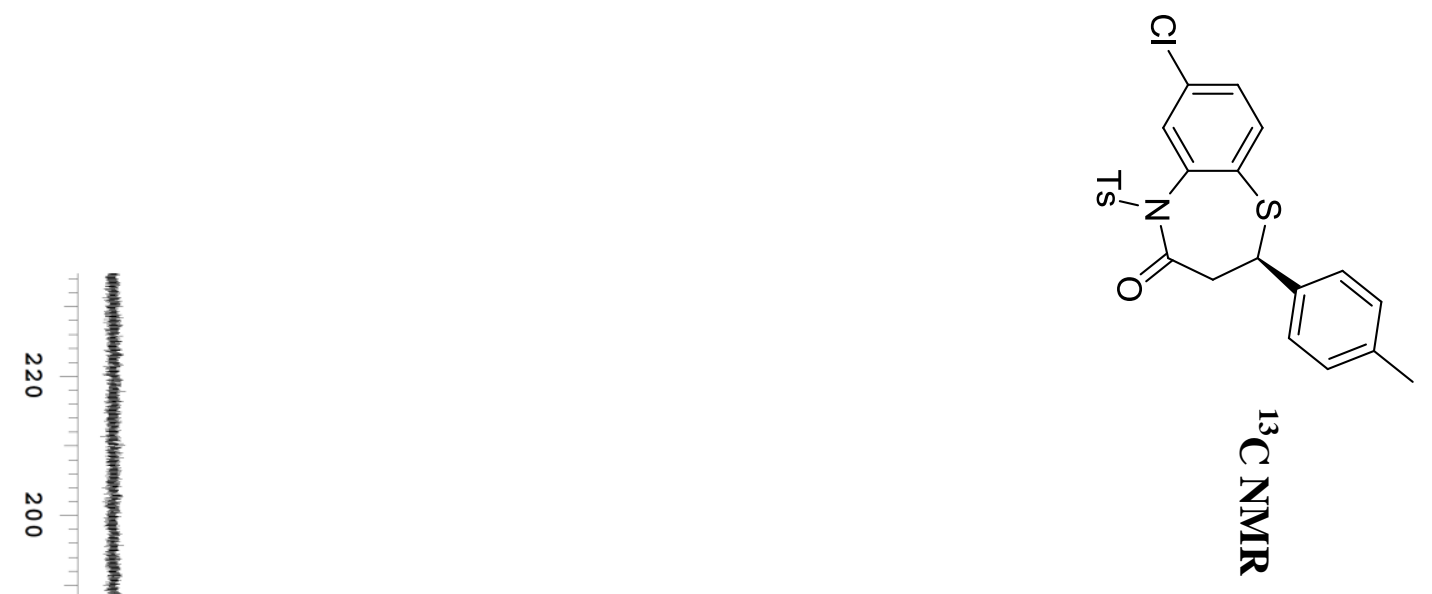

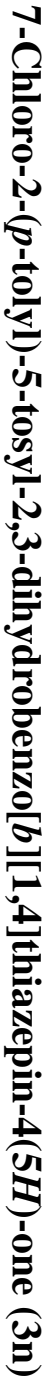

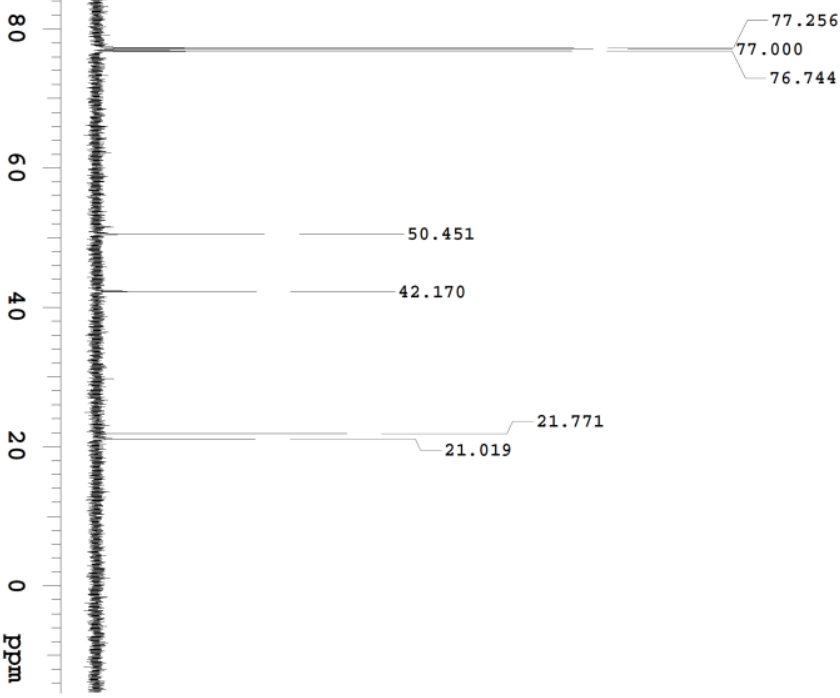



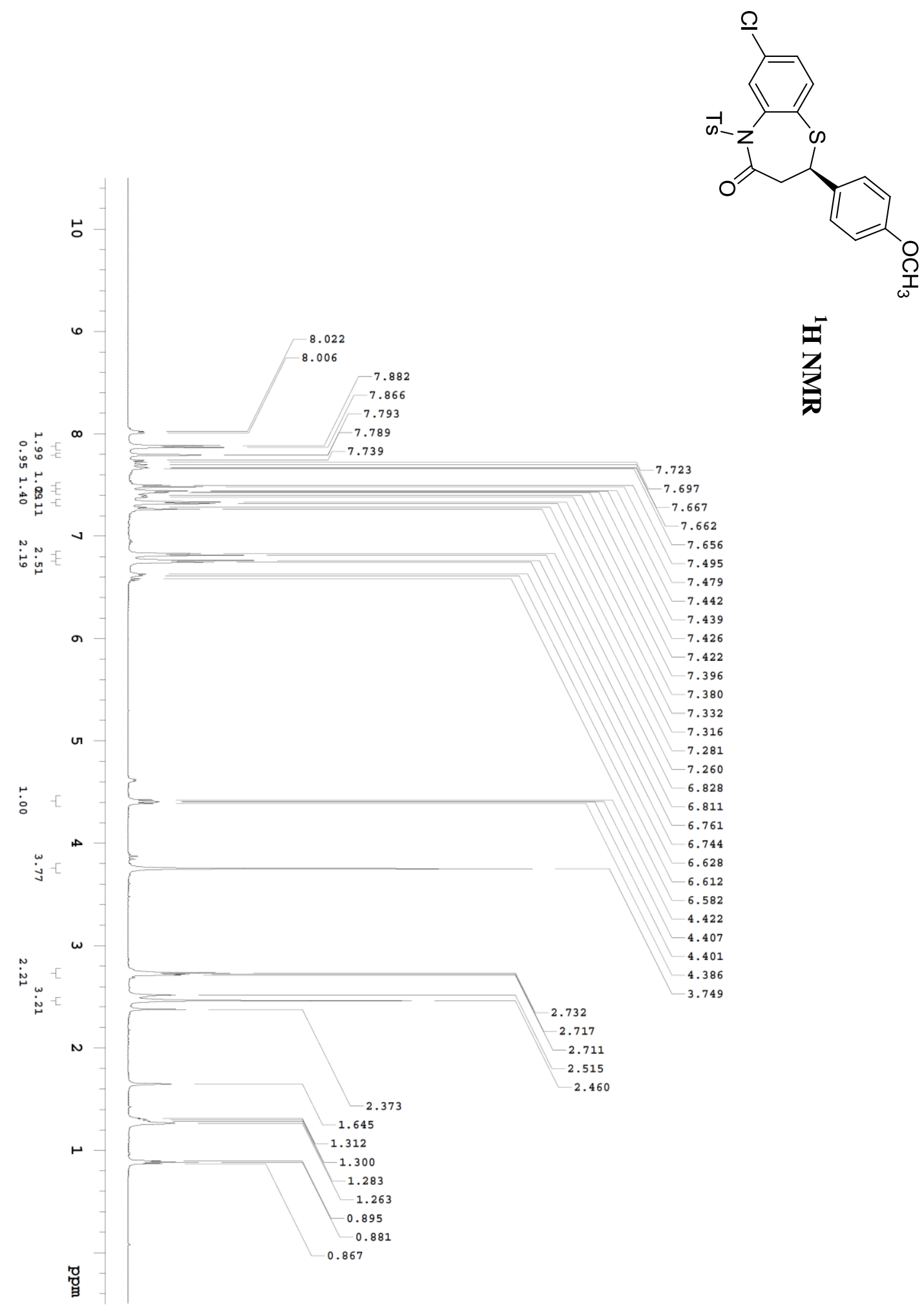

年

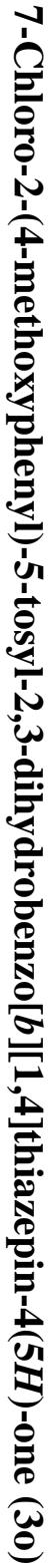



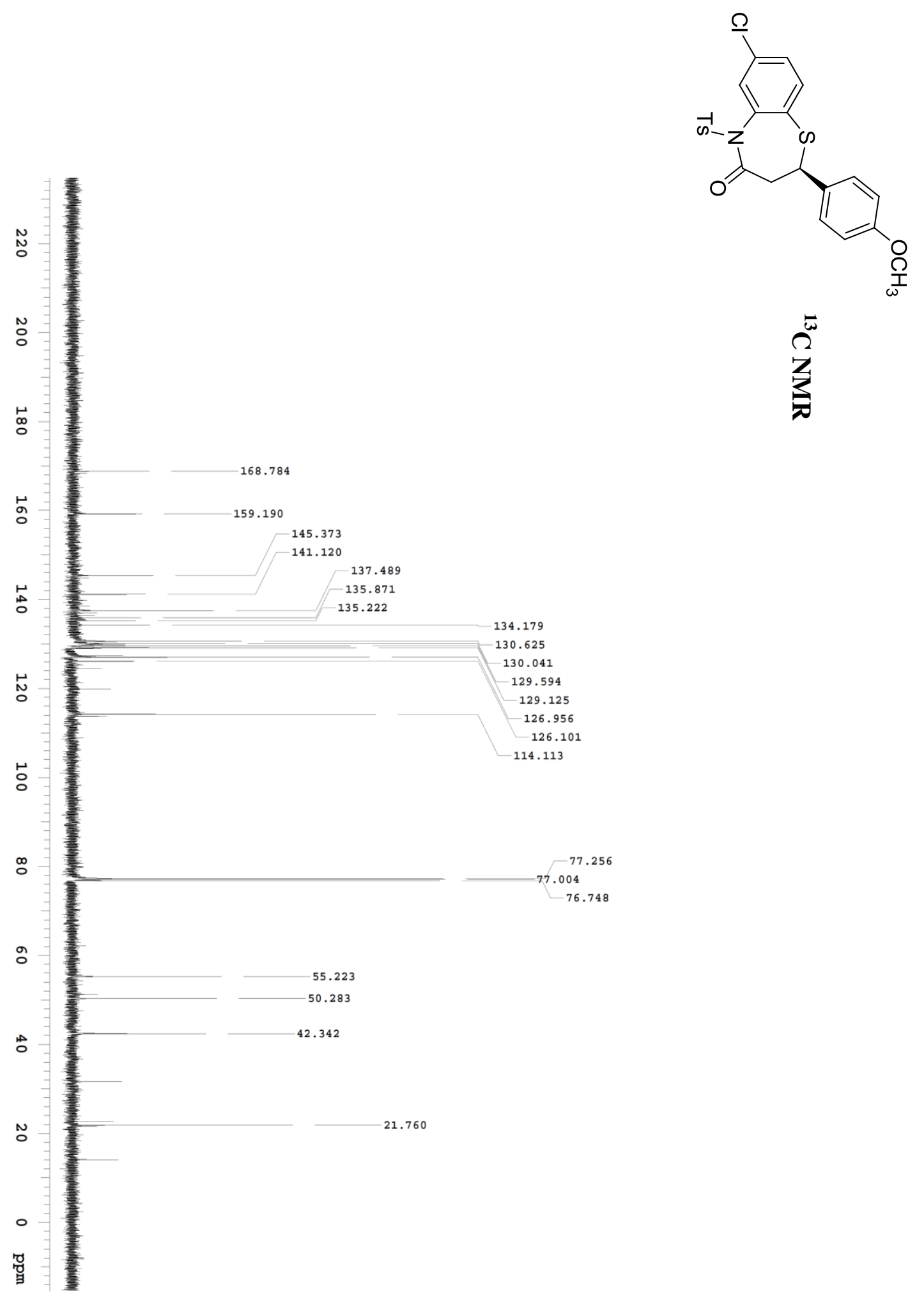

(3)

${ }^{2}$

ل. 

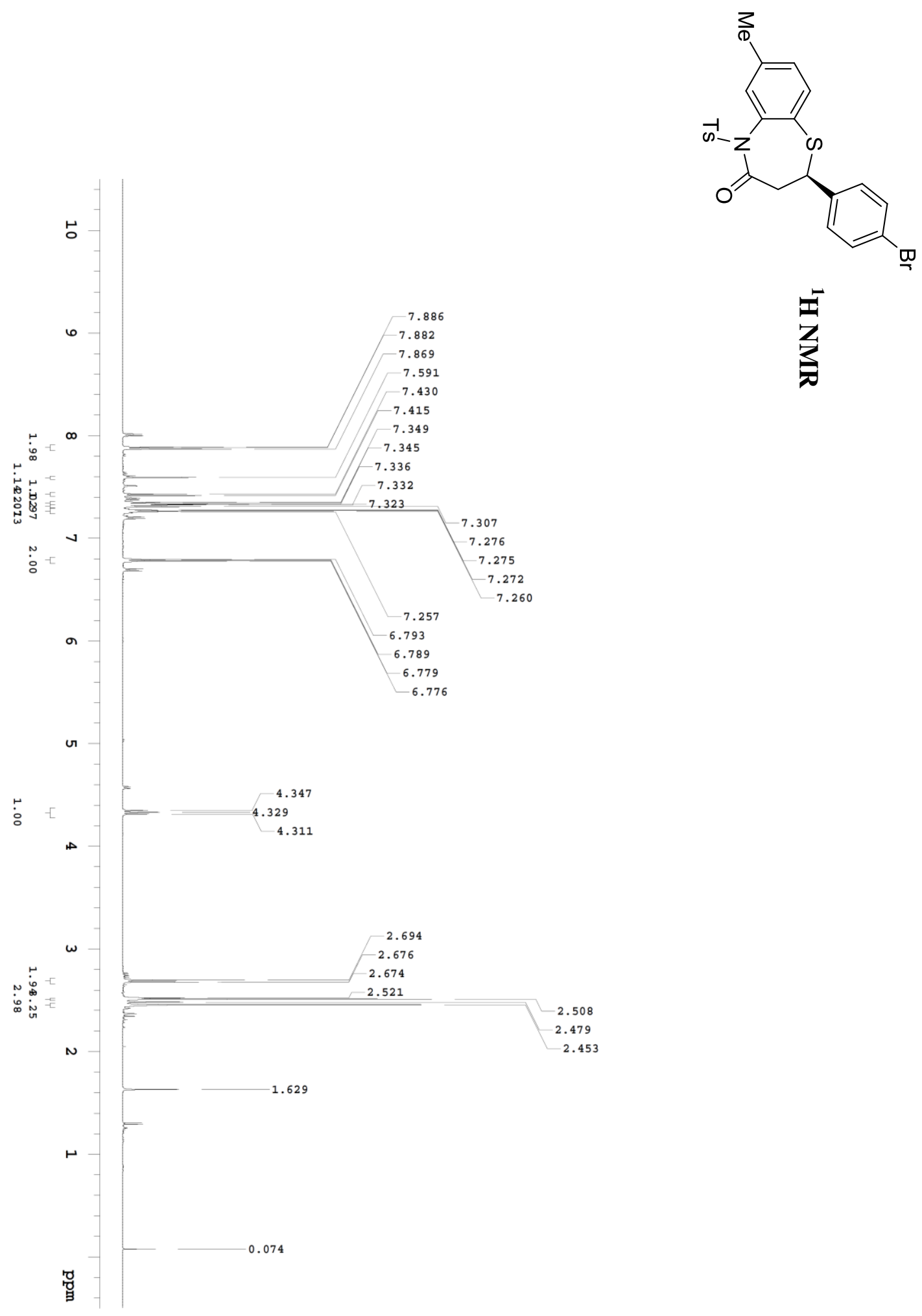

مُ

$\frac{7}{2}$

它 

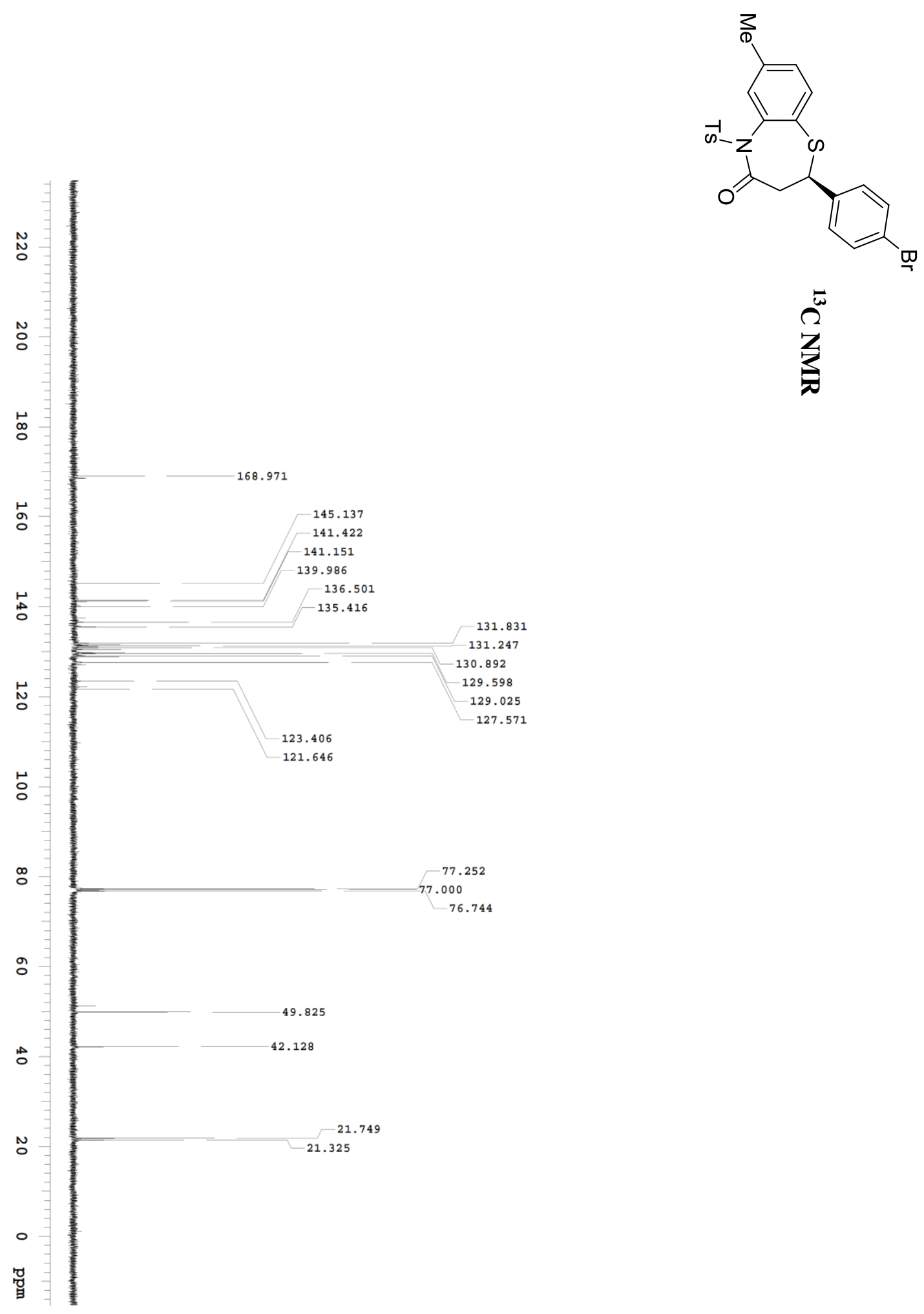

مُ

$\overbrace{}^{2}$

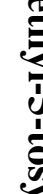



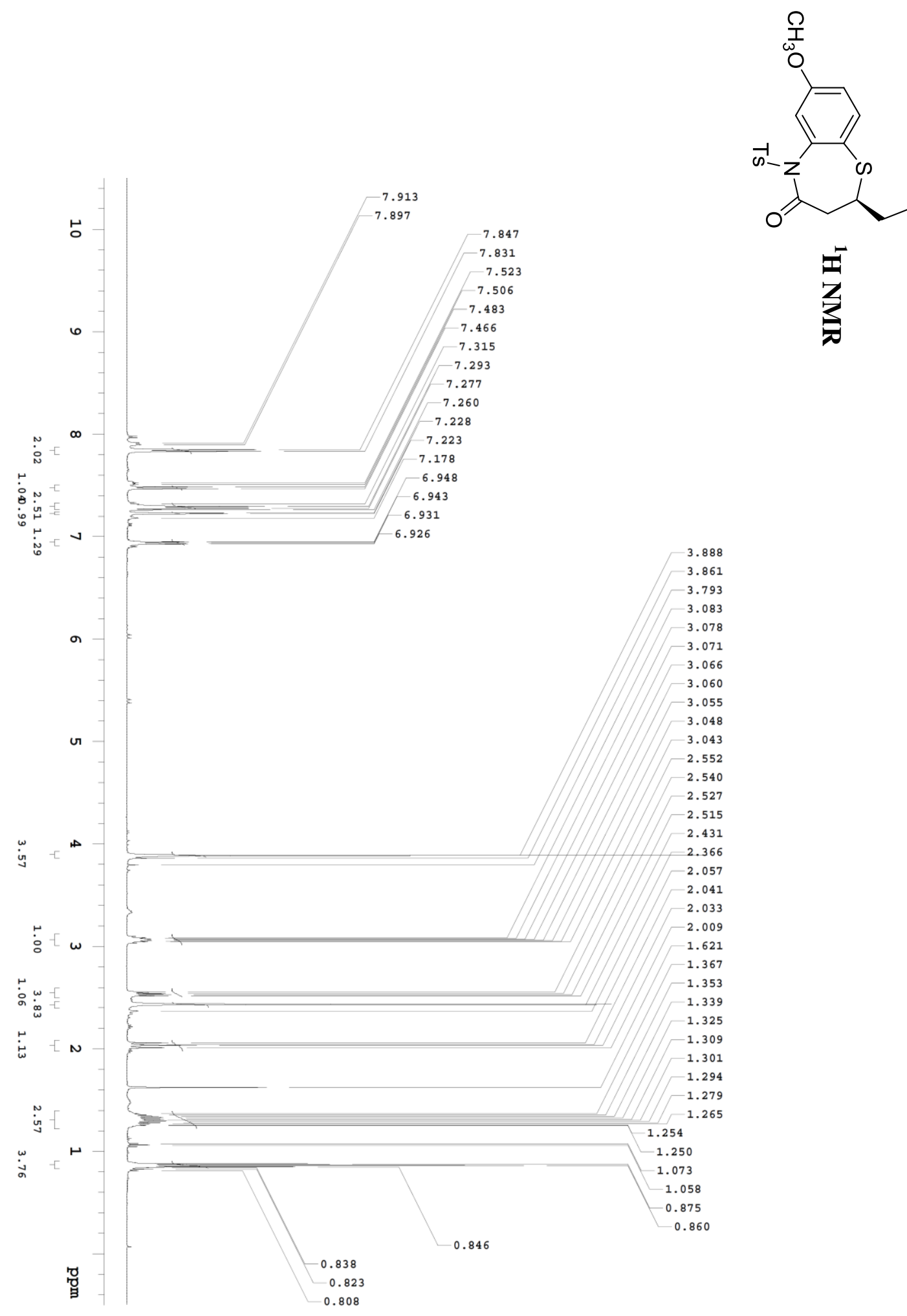

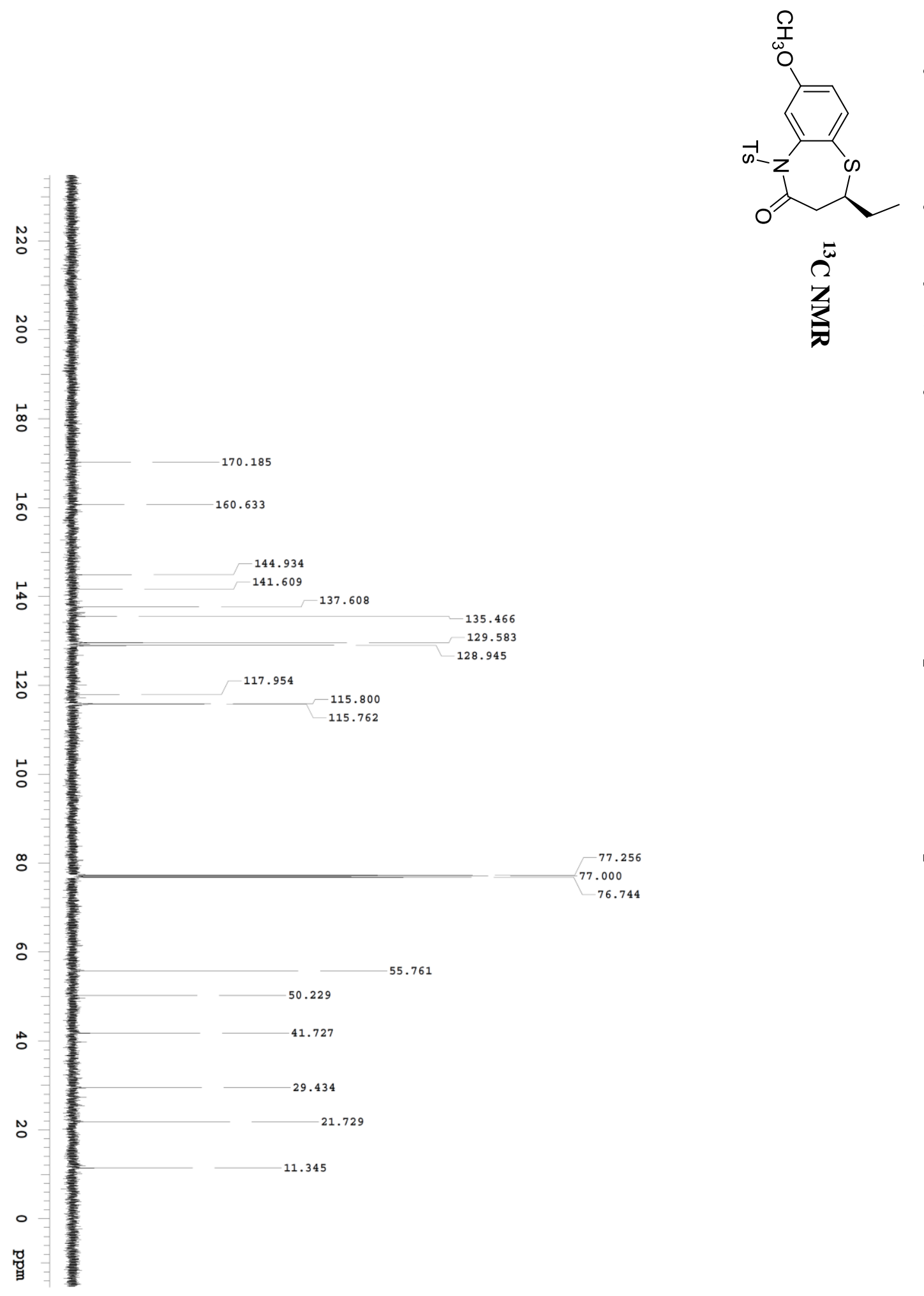

1'

光

ฝั

:

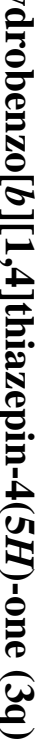




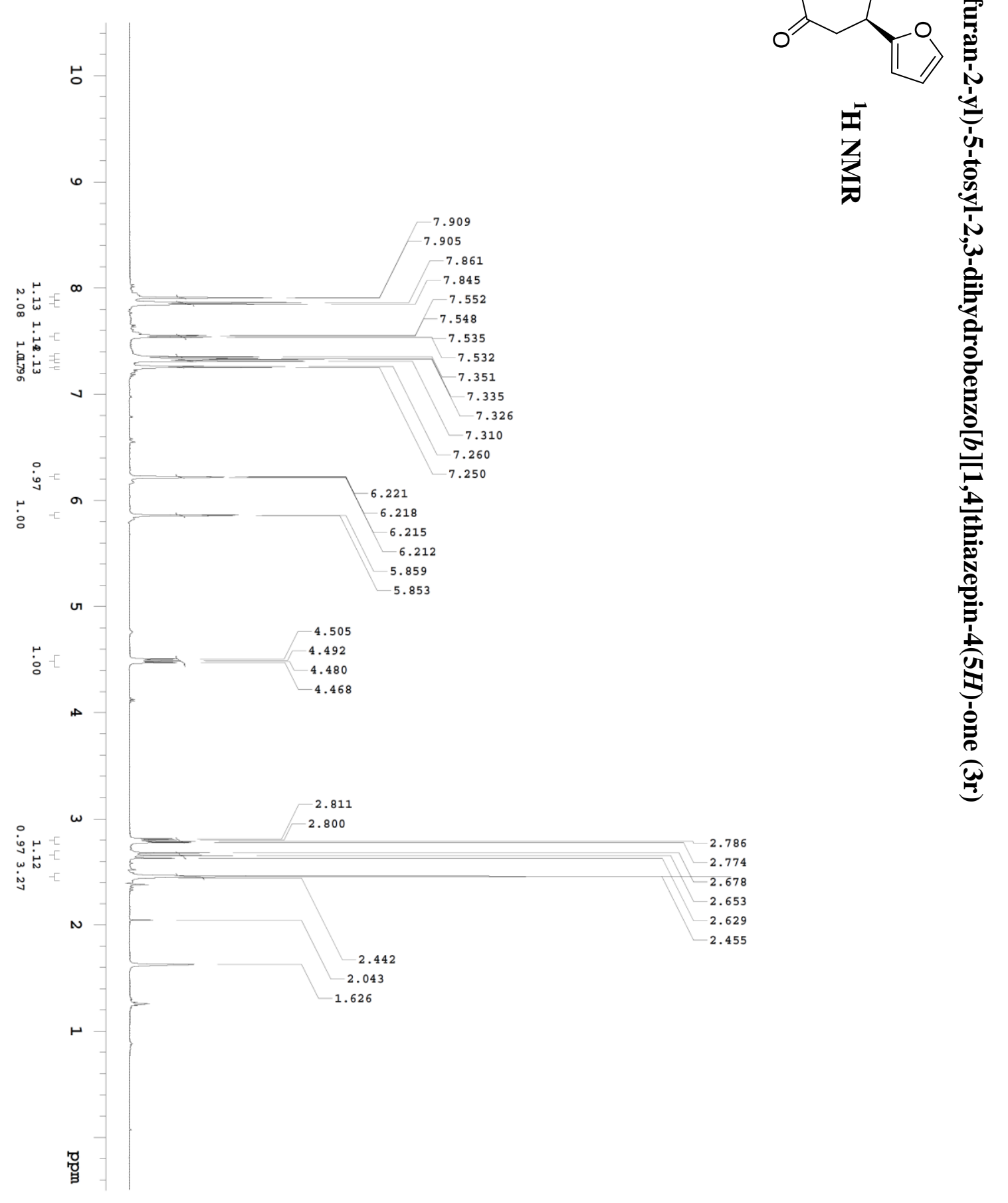



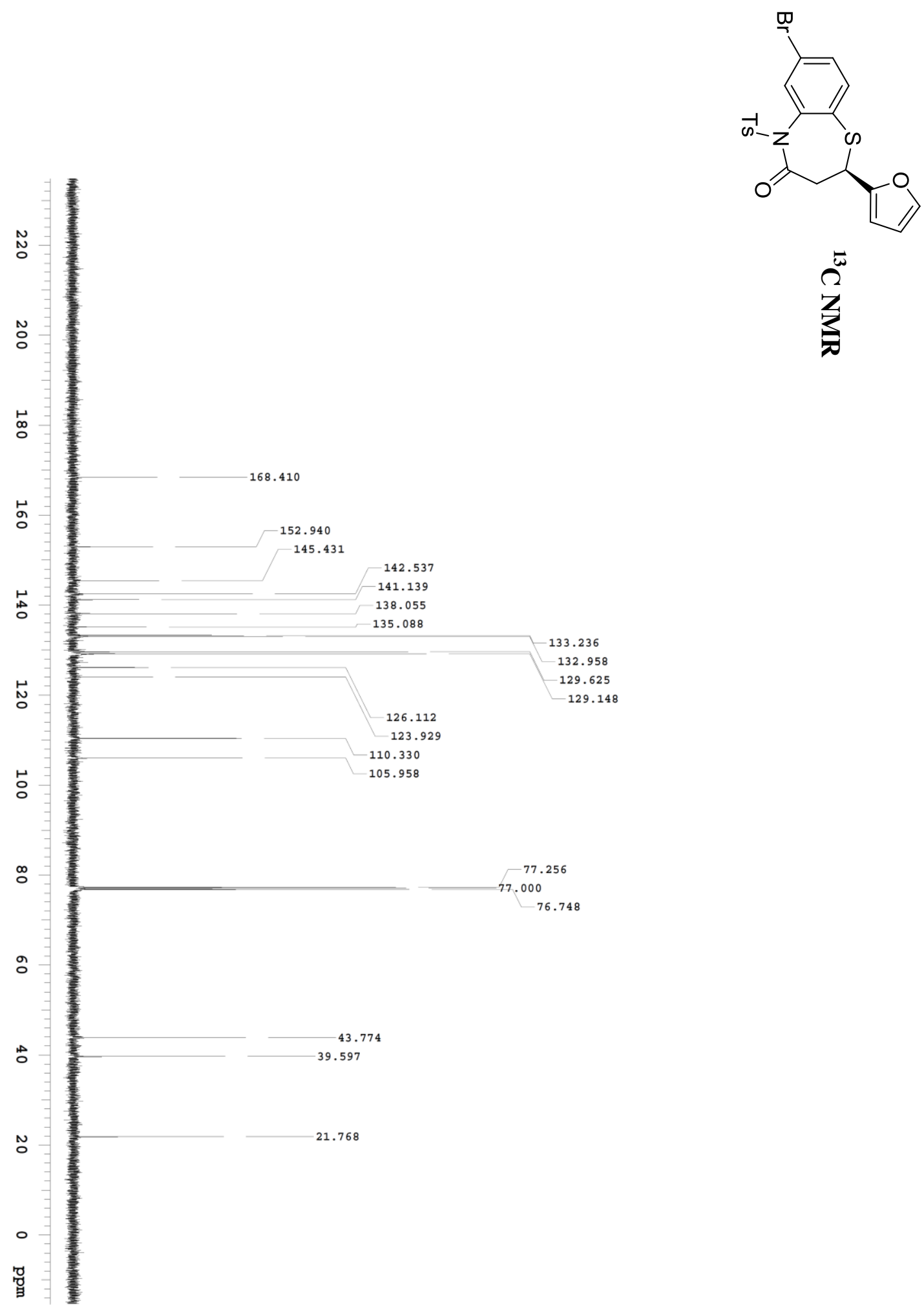

نُ

를 

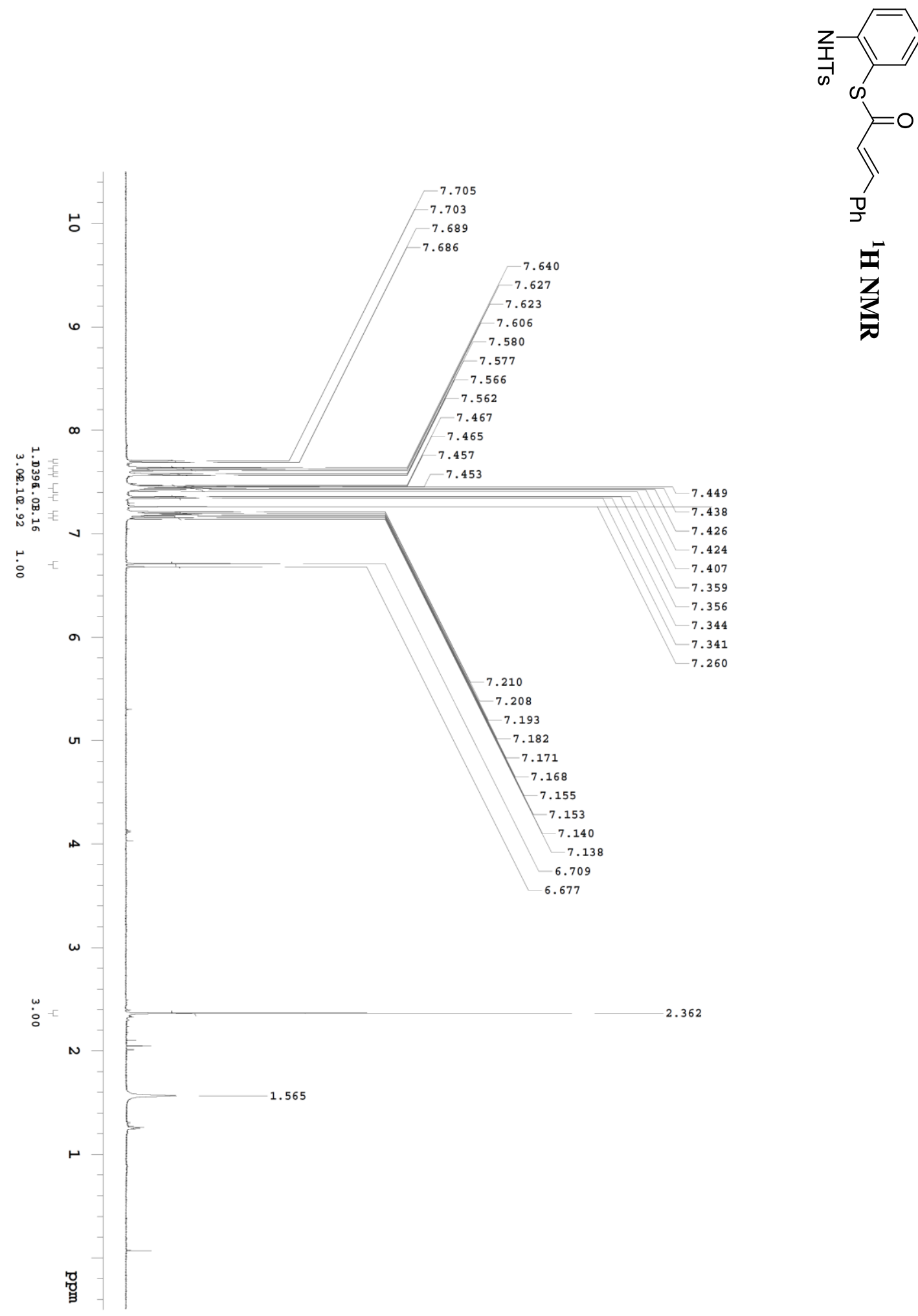

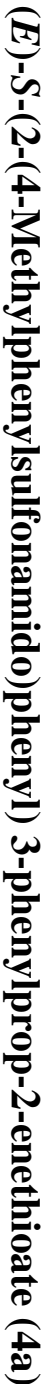




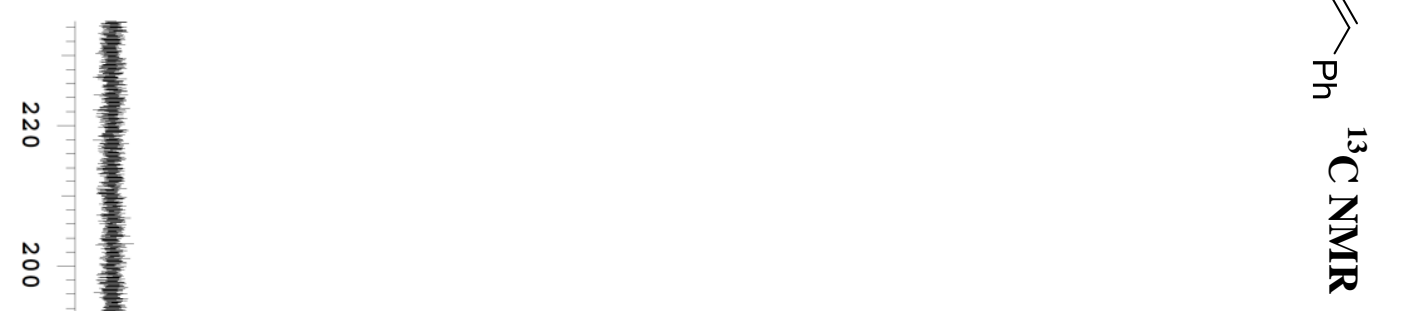

飠

농

落

$-143.178$ $-138.833$

$-136.764$

136.020
-133.542

131.621

29.636

129.132
-128.670

$-127.284$

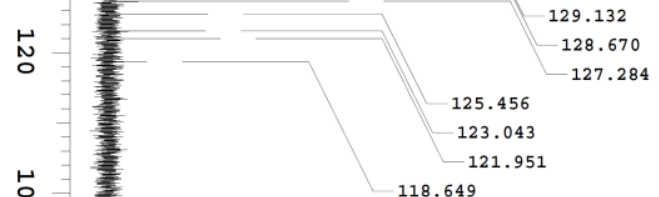

$\stackrel{\infty}{\circ}$

118.649

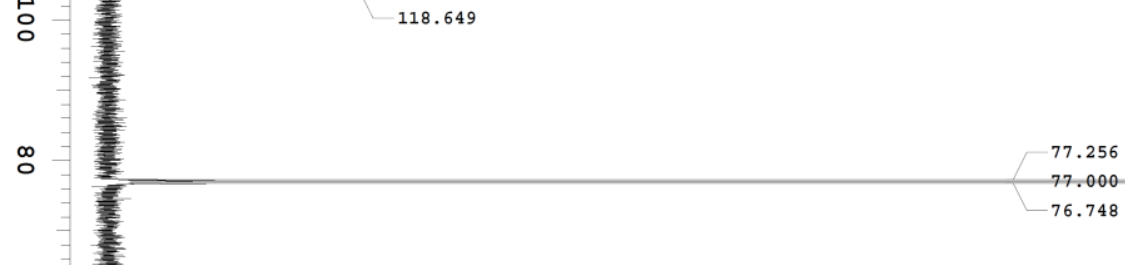




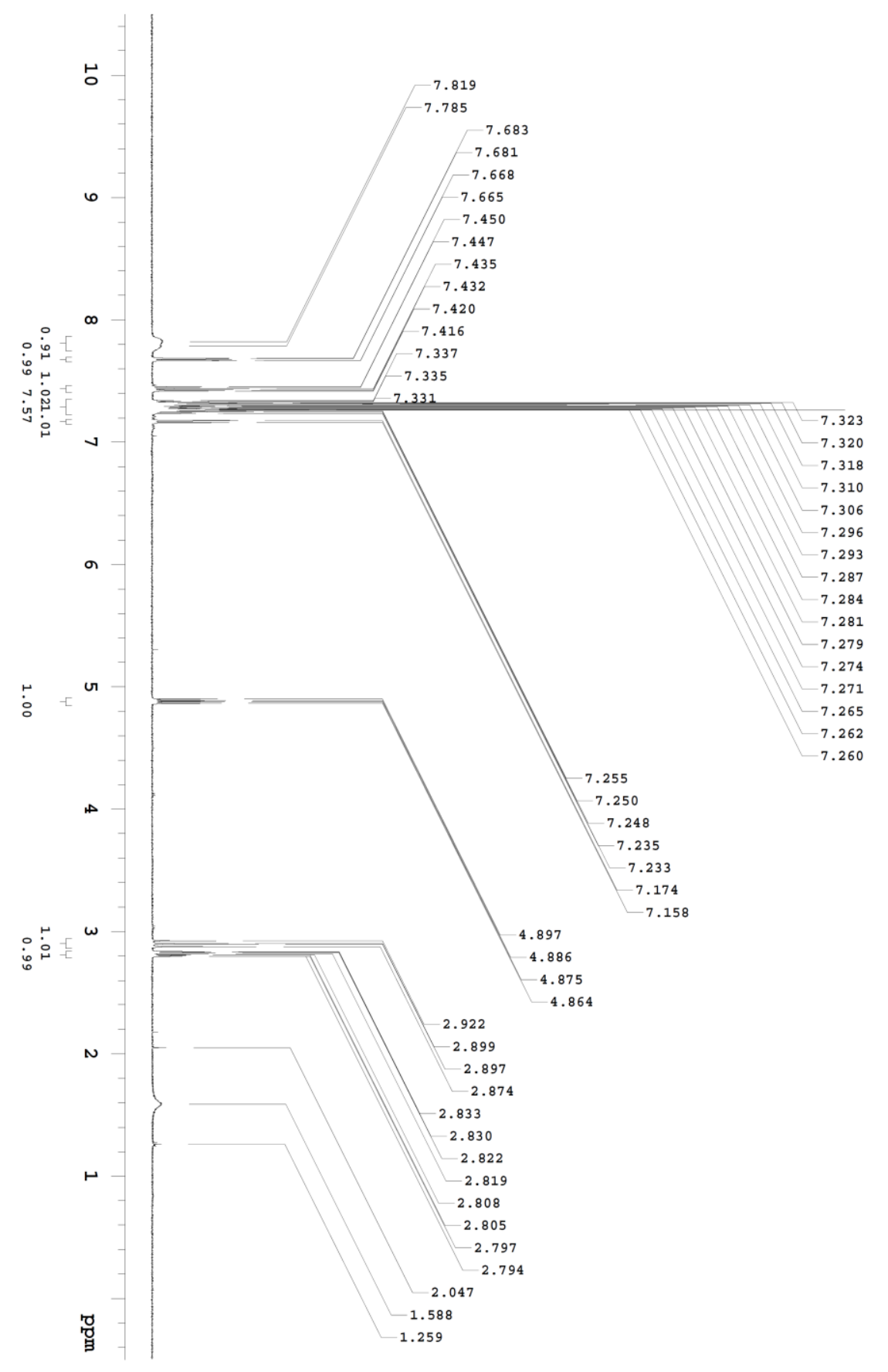



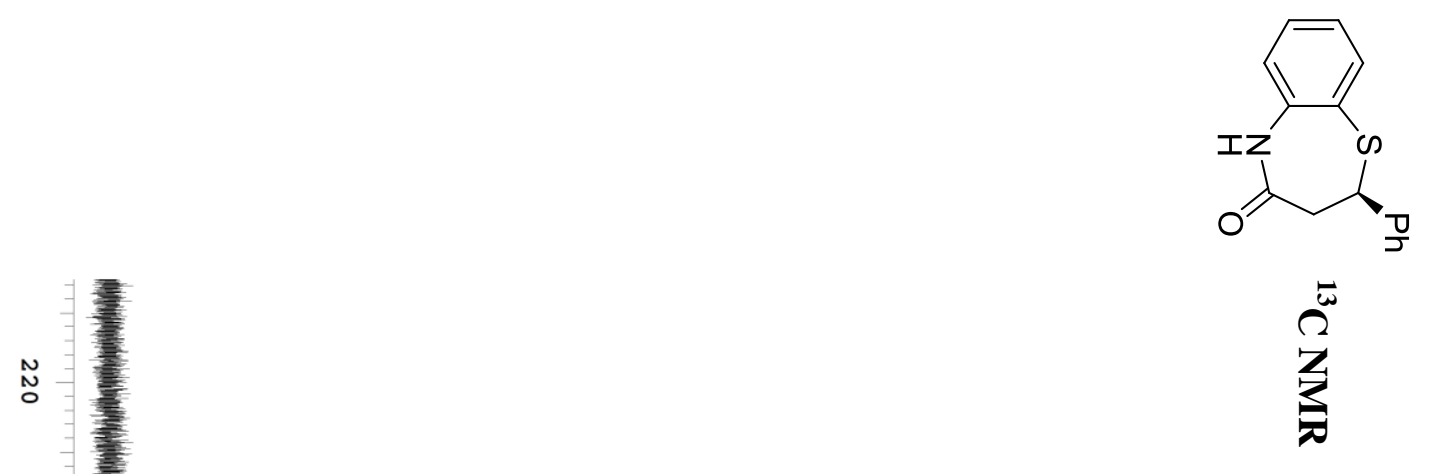

ָِ

:

$\stackrel{⿱ ⺊ 口}{\circ}$

농

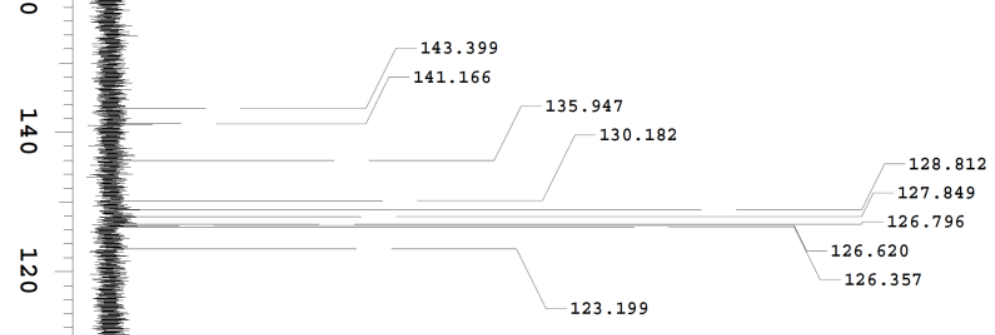

$-77.256$

है.

吕

$\stackrel{\infty}{\circ}$

옹

N

○

䍃

53.066

$-41.433$ 


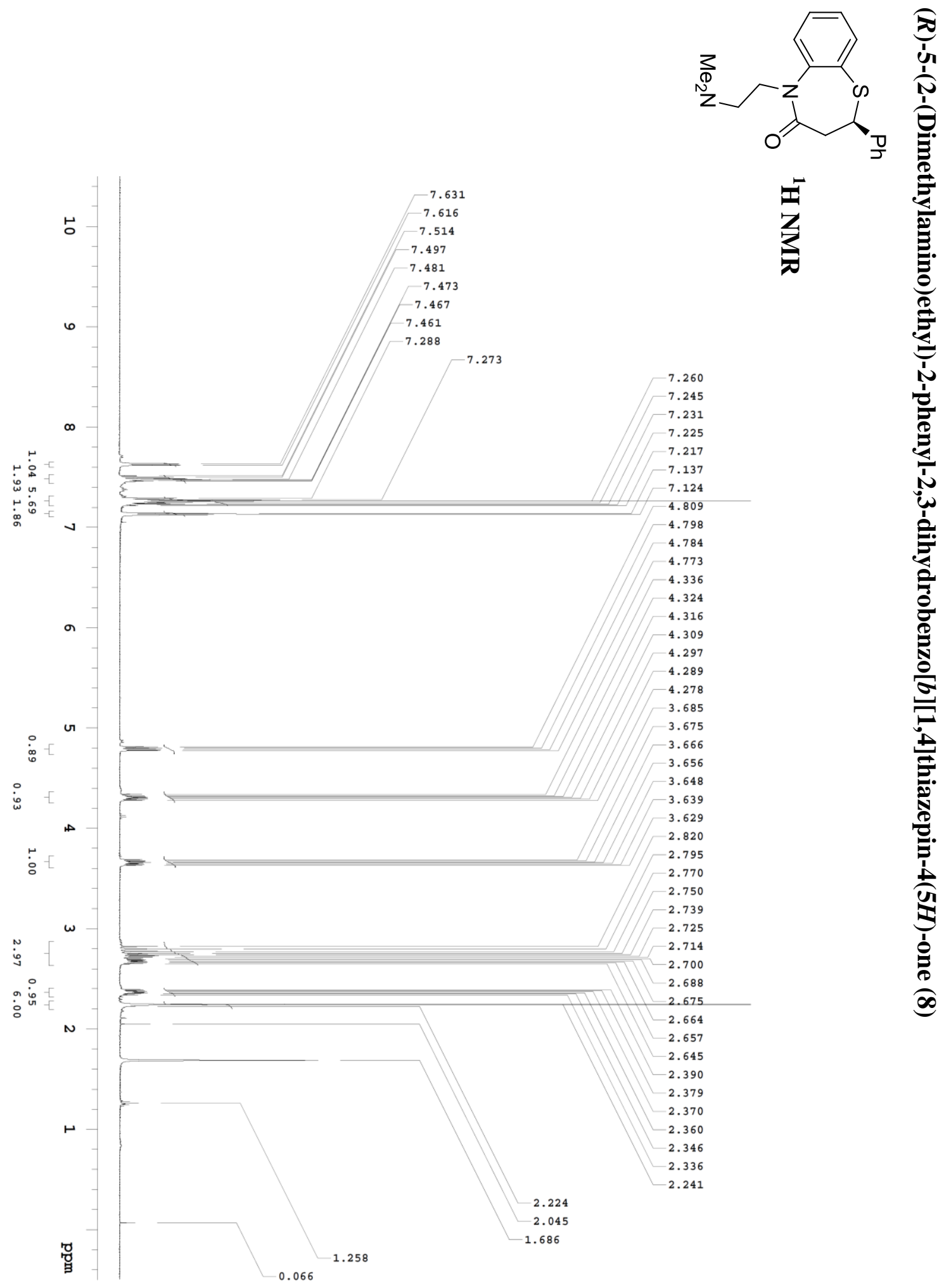




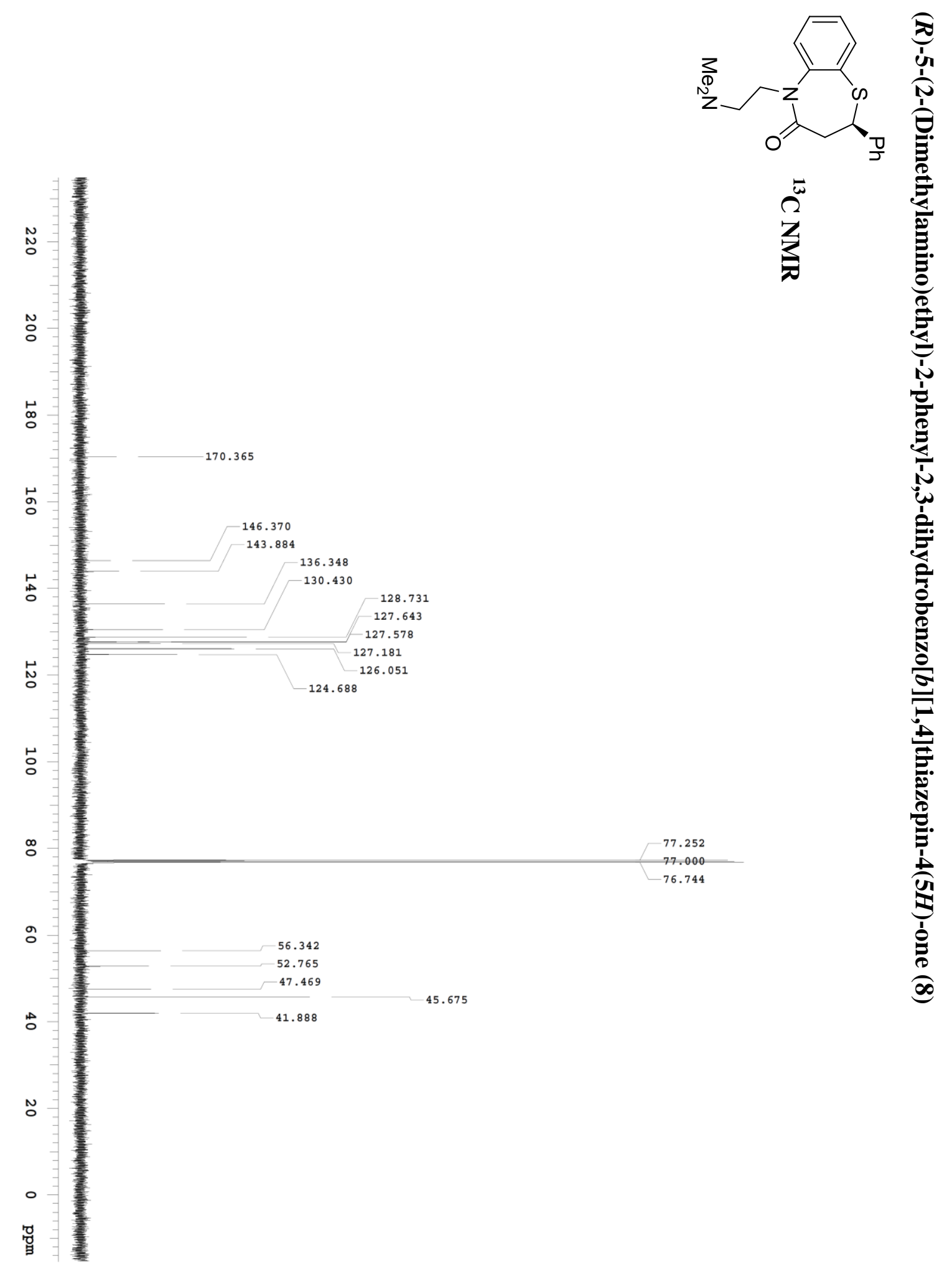




\section{HPLC Chromatogram Plofiles}

(R)-2-Phenyl-5-tosyl-2,3-dihydrobenzo[b][1,4]thiazepin-4(5H)-one (3a).

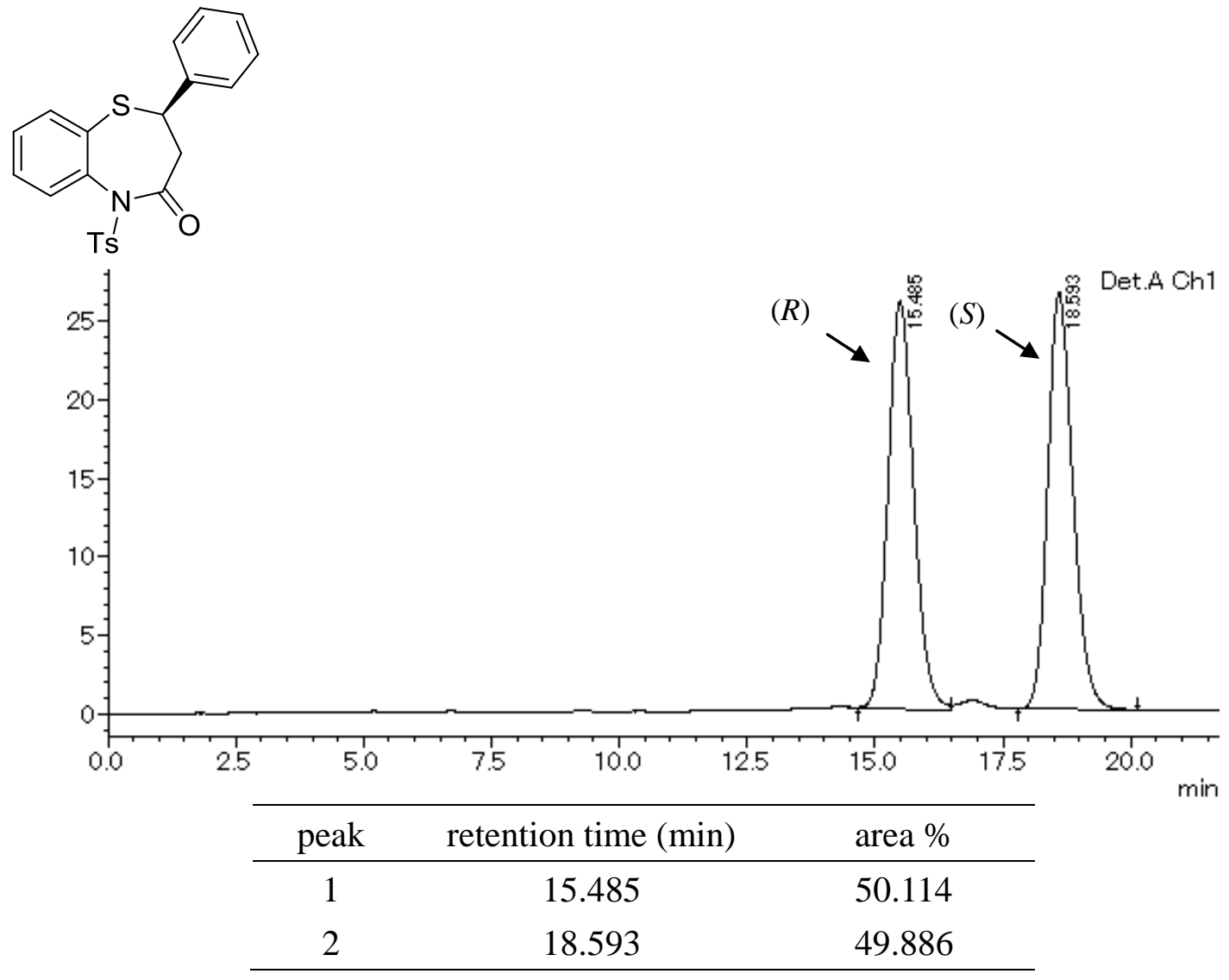

Product from (E)-1a: 97\% ee.

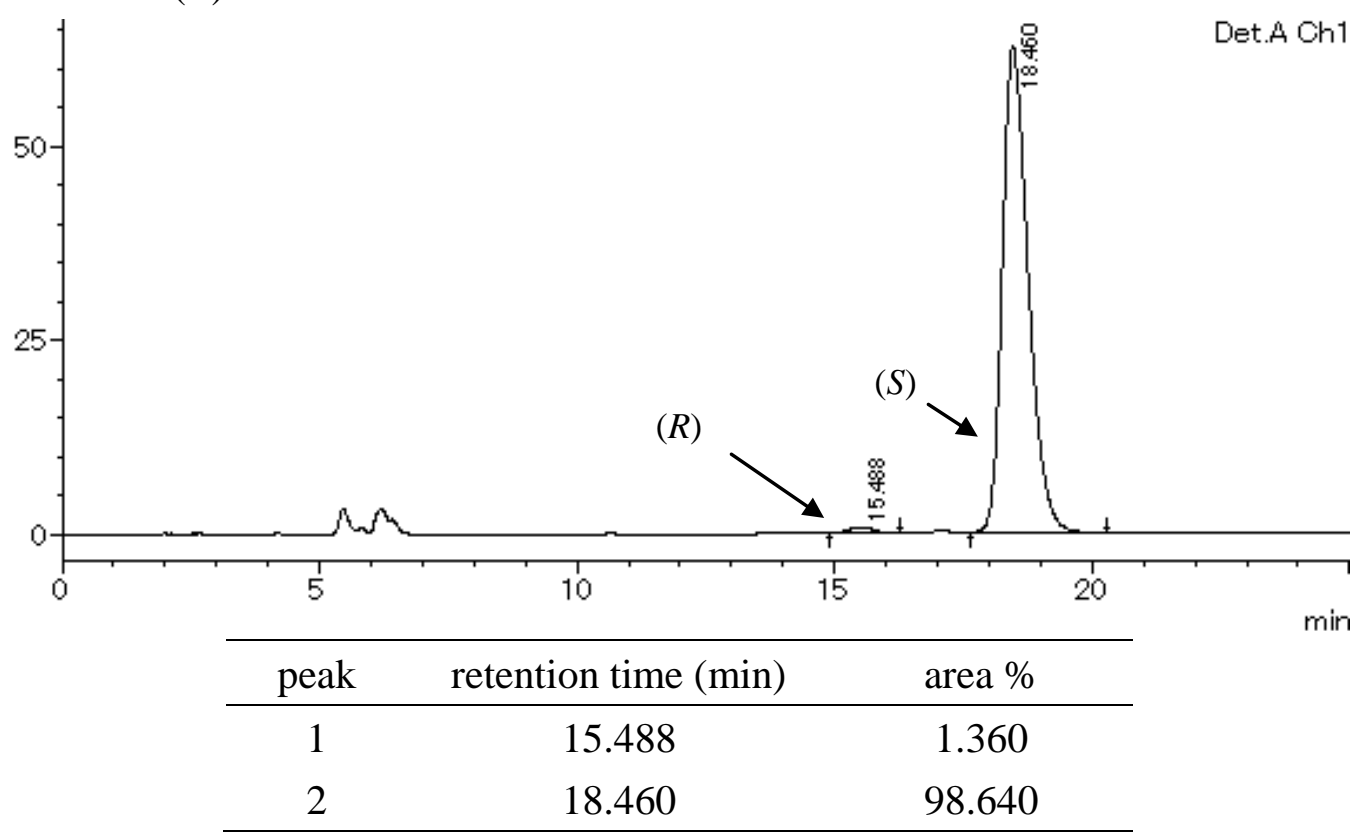


Product from (Z)-1a : 97\% ee.

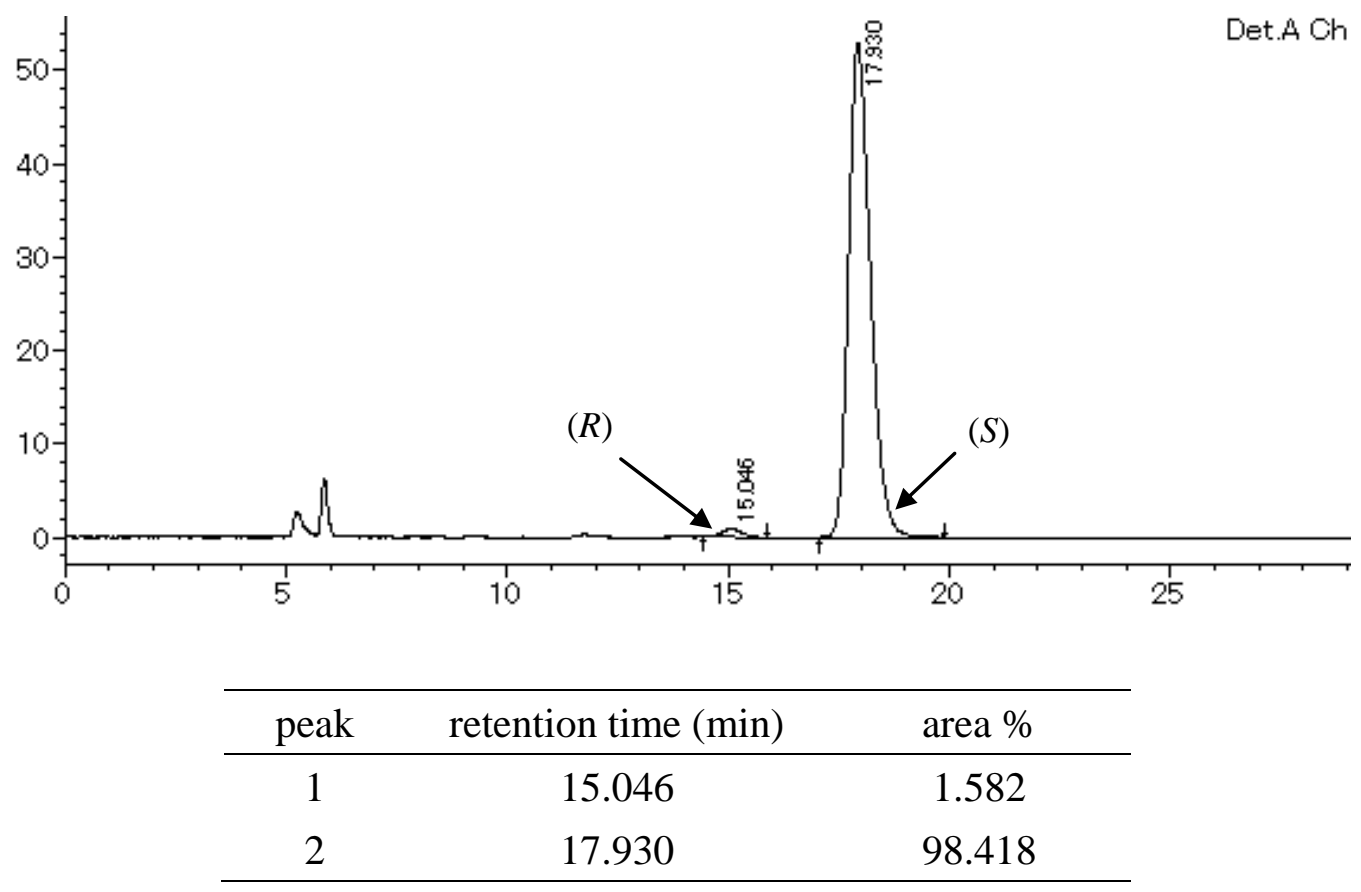


7-Methyl-2-phenyl-5-tosyl-2,3-dihydrobenzo[b][1,4]thiazepin-4(5H)-one (3b).<smiles>Cc1ccc2c(c1)N([13S])C(=O)CC(c1ccccc1)S2</smiles>
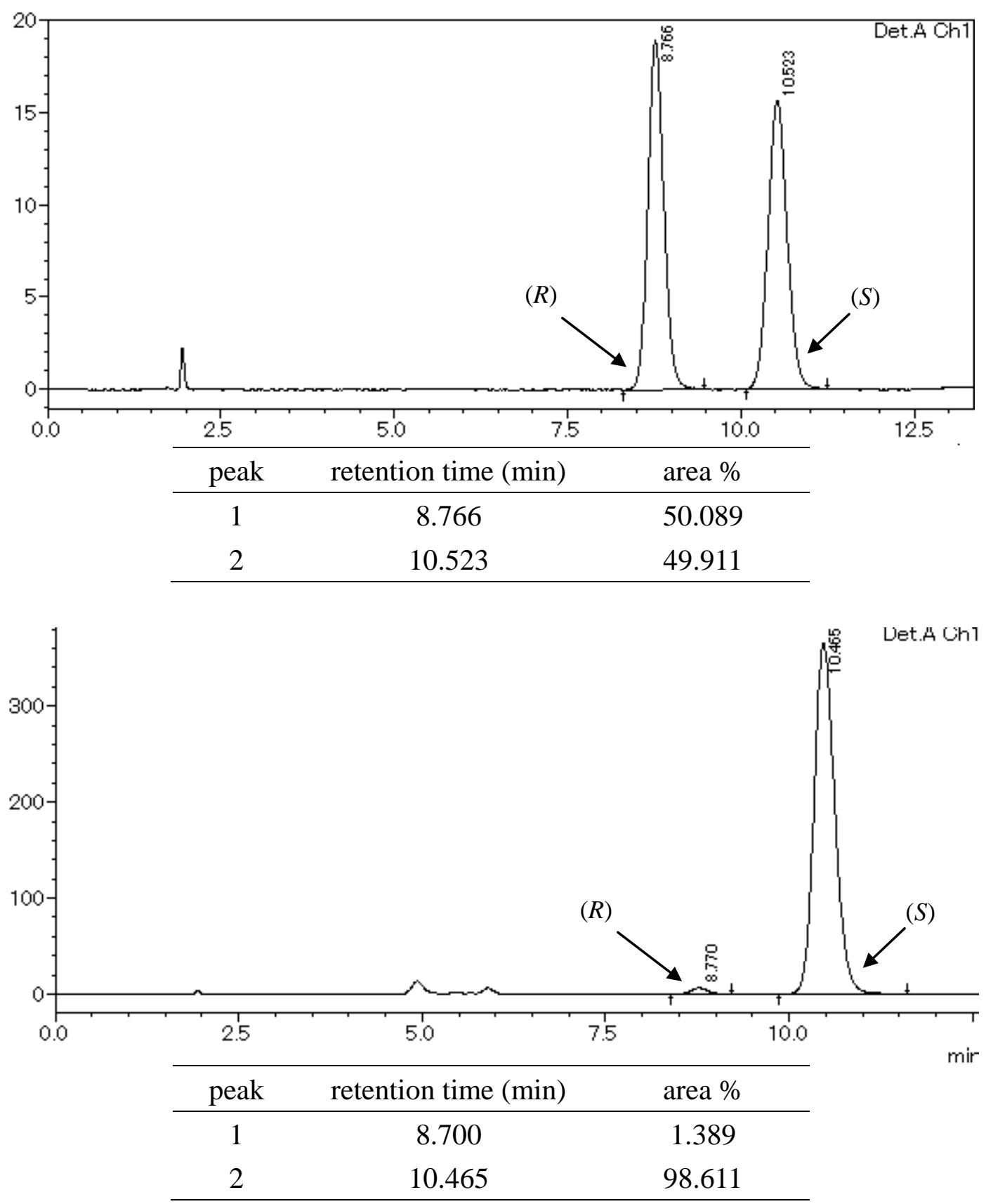
7-Methoxy-2-phenyl-5-tosyl-2,3-dihydrobenzo $[b][1,4]$ thiazepin-4(5H)-one $(3 \mathrm{c})$.<smiles>COc1ccc2c(c1)N([As])C(=O)CC(c1ccccc1)S2</smiles>

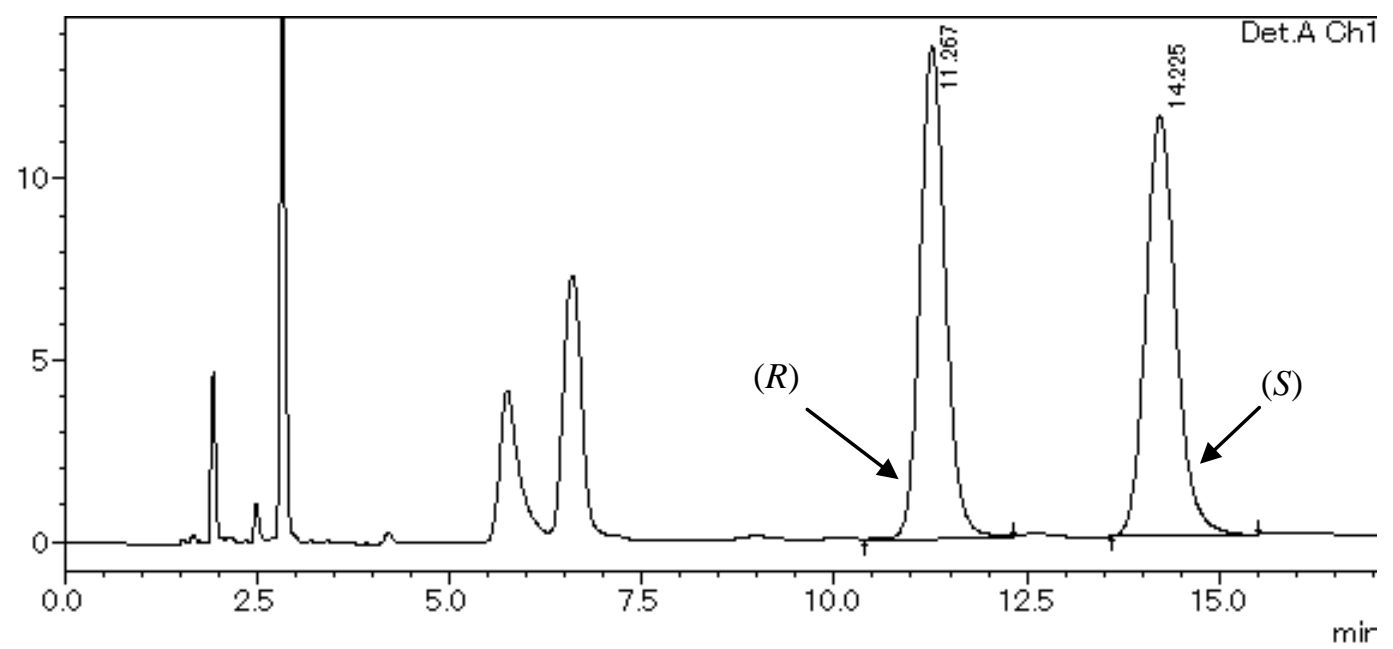

\begin{tabular}{ccc}
\hline peak & retention time $(\min )$ & area \% \\
\hline 1 & 11.267 & 49.809 \\
2 & 14.225 & 50.191 \\
\hline
\end{tabular}

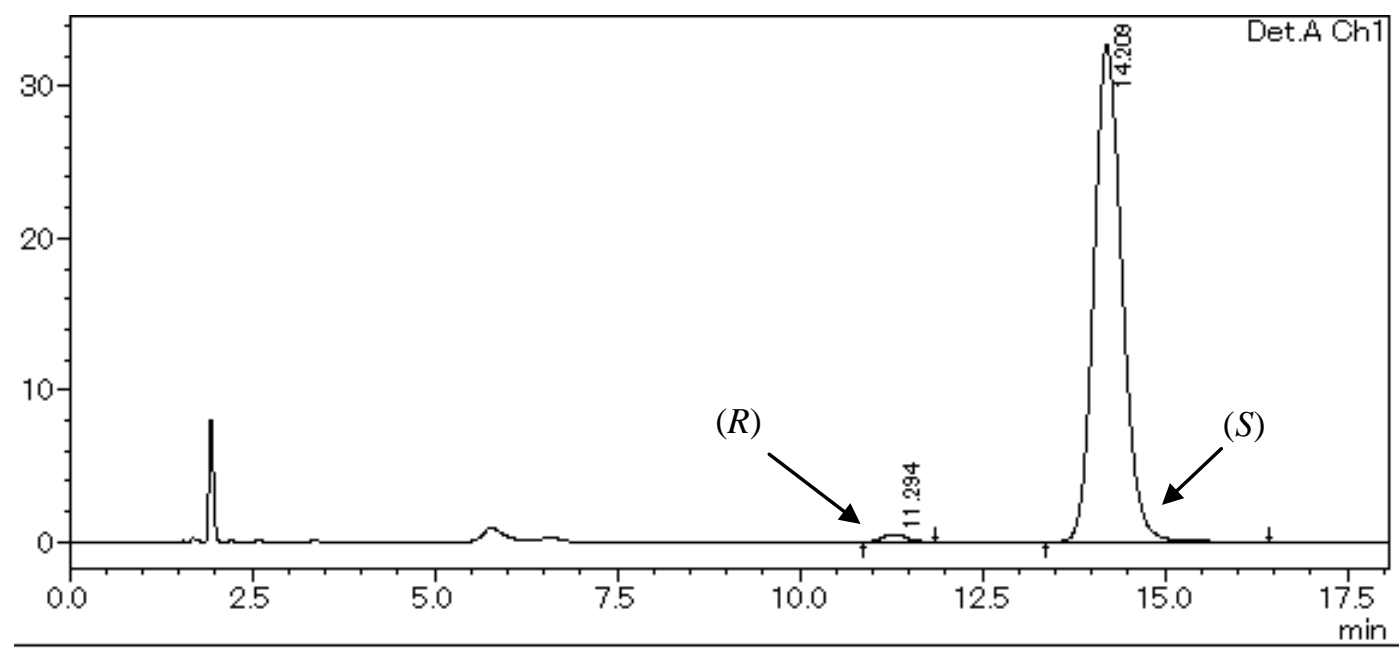

\begin{tabular}{ccc}
\hline peak & retention time $(\min )$ & area $\%$ \\
\hline 1 & 11.294 & 1.238 \\
2 & 14.209 & 98.762 \\
\hline
\end{tabular}


7-Chloro-2-phenyl-5-tosyl-2,3-dihydrobenzo $[b][1,4]$ thiazepin-4(5H)-one (3d).<smiles>O=C1C[C@@H](c2ccccc2)Sc2ccc(Cl)cc2N1[13S]</smiles>

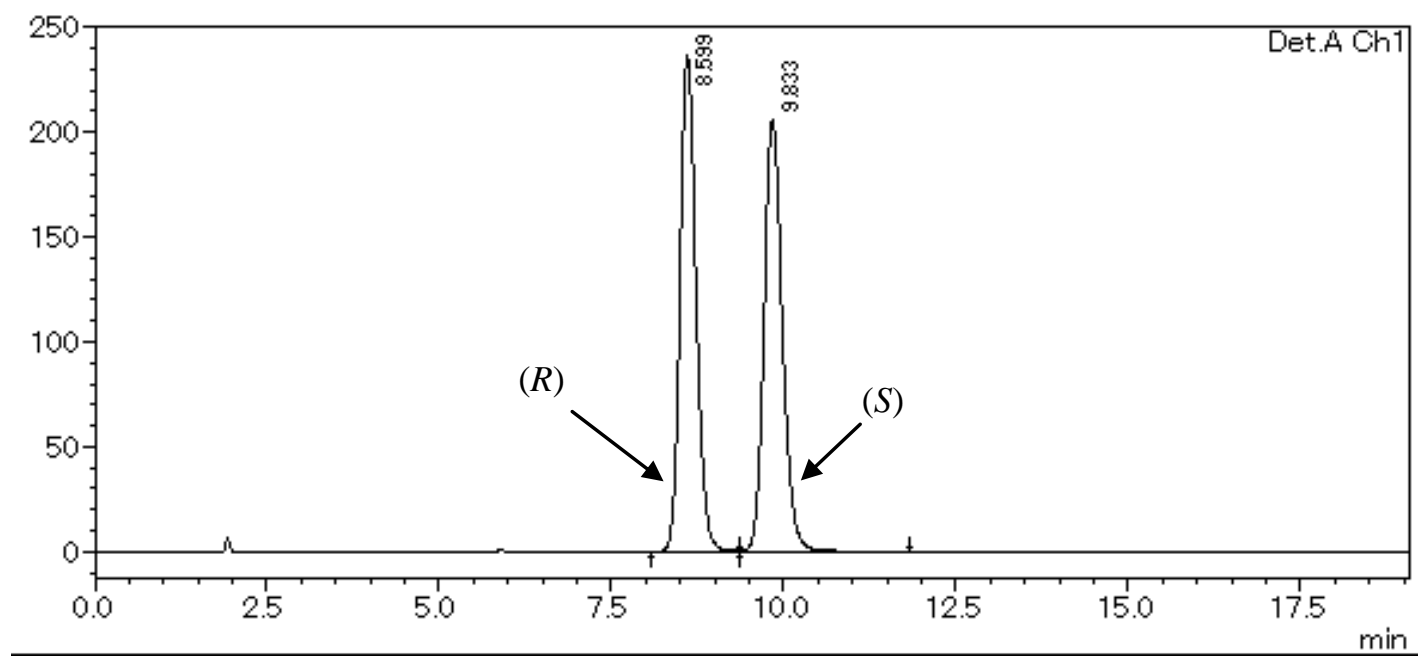

\begin{tabular}{ccc}
\hline peak & retention time $(\min )$ & area $\%$ \\
\hline 1 & 8.599 & 50.105 \\
2 & 9.833 & 49.895 \\
\hline
\end{tabular}

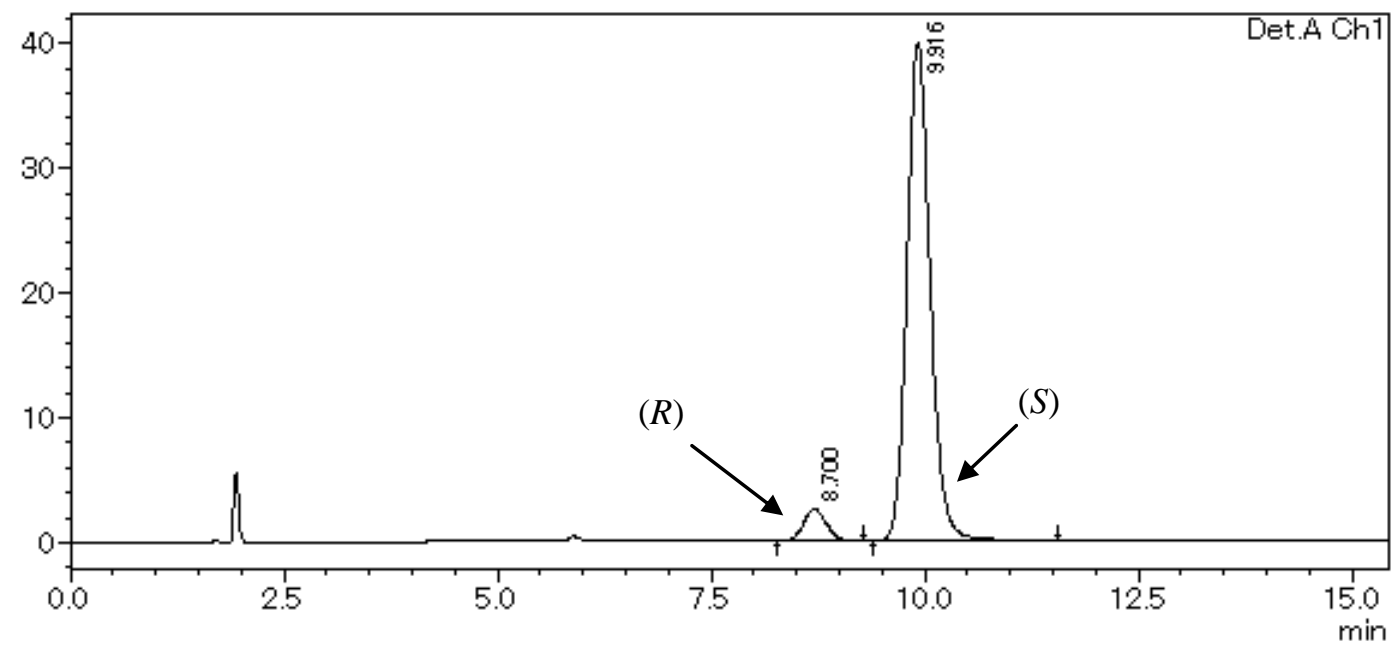

\begin{tabular}{ccc}
\hline peak & retention time $(\min )$ & area $\%$ \\
\hline 1 & 8.700 & 5.830 \\
2 & 9.916 & 94.170 \\
\hline
\end{tabular}


7-Bromo-2-phenyl-5-tosyl-2,3-dihydrobenzo $[b][1,4]$ thiazepin-4(5H)-one $(3 e)$.<smiles>[3H]N1C(=O)C[C@@H](c2ccccc2)Sc2ccc(Br)cc21</smiles>

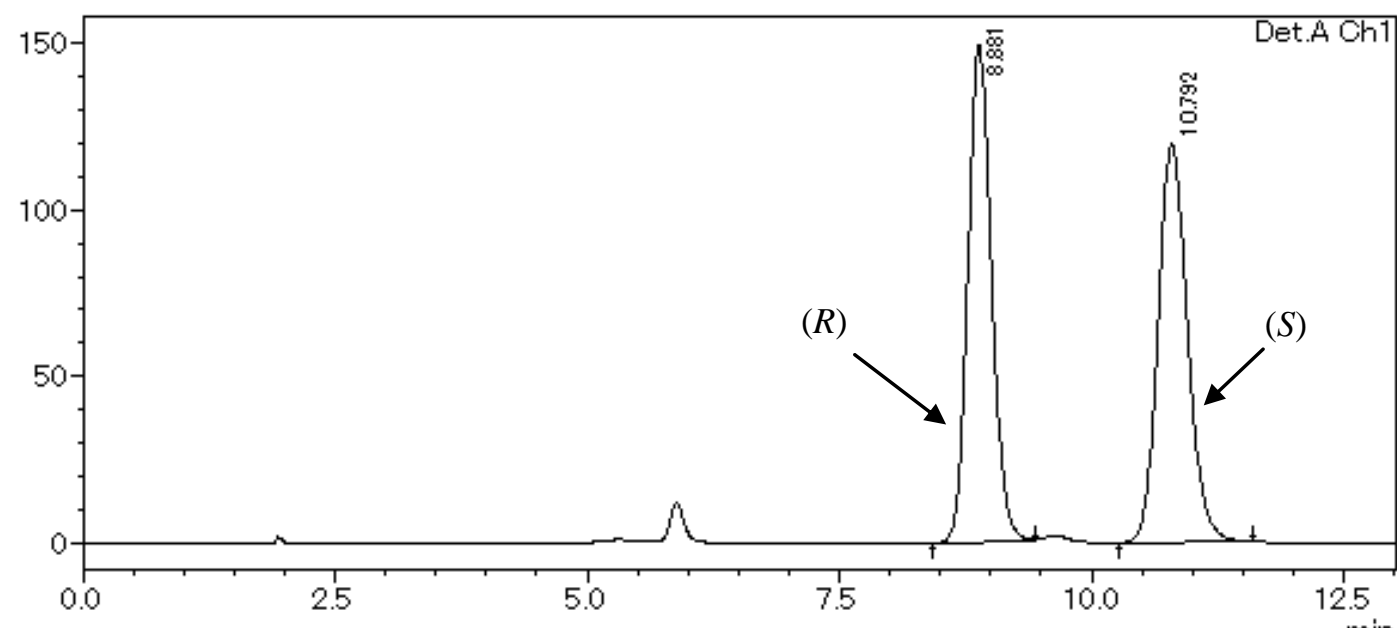

\begin{tabular}{ccc}
\hline peak & retention time $(\min )$ & area $\%$ \\
\hline 1 & 8.881 & 50.130 \\
2 & 10.792 & 49.870 \\
\hline
\end{tabular}

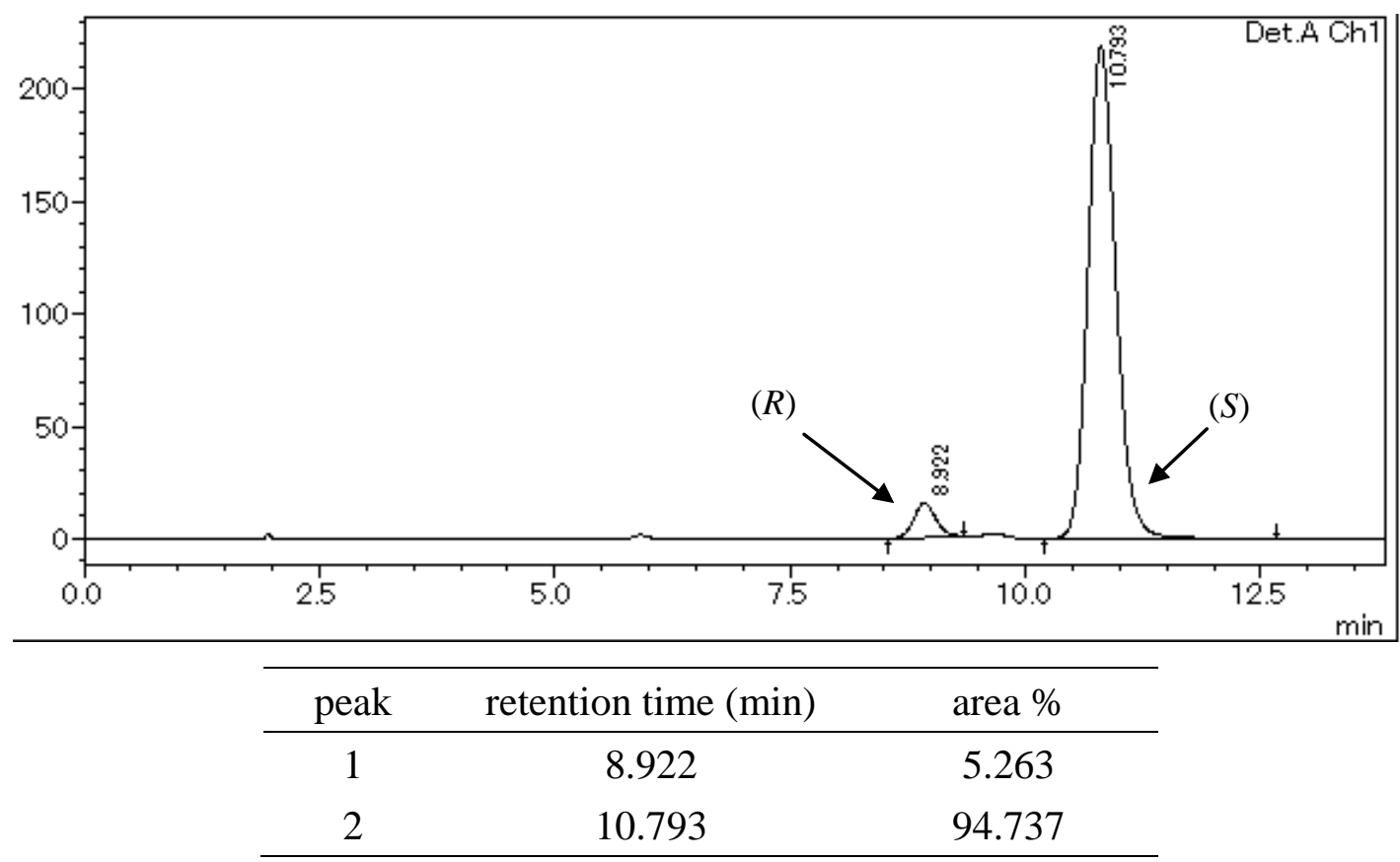


2-(4-Methylphenyl)-5-tosyl-2,3-dihydrobenzo[b][1,4]thiazepin-4(5H)-one (3f).
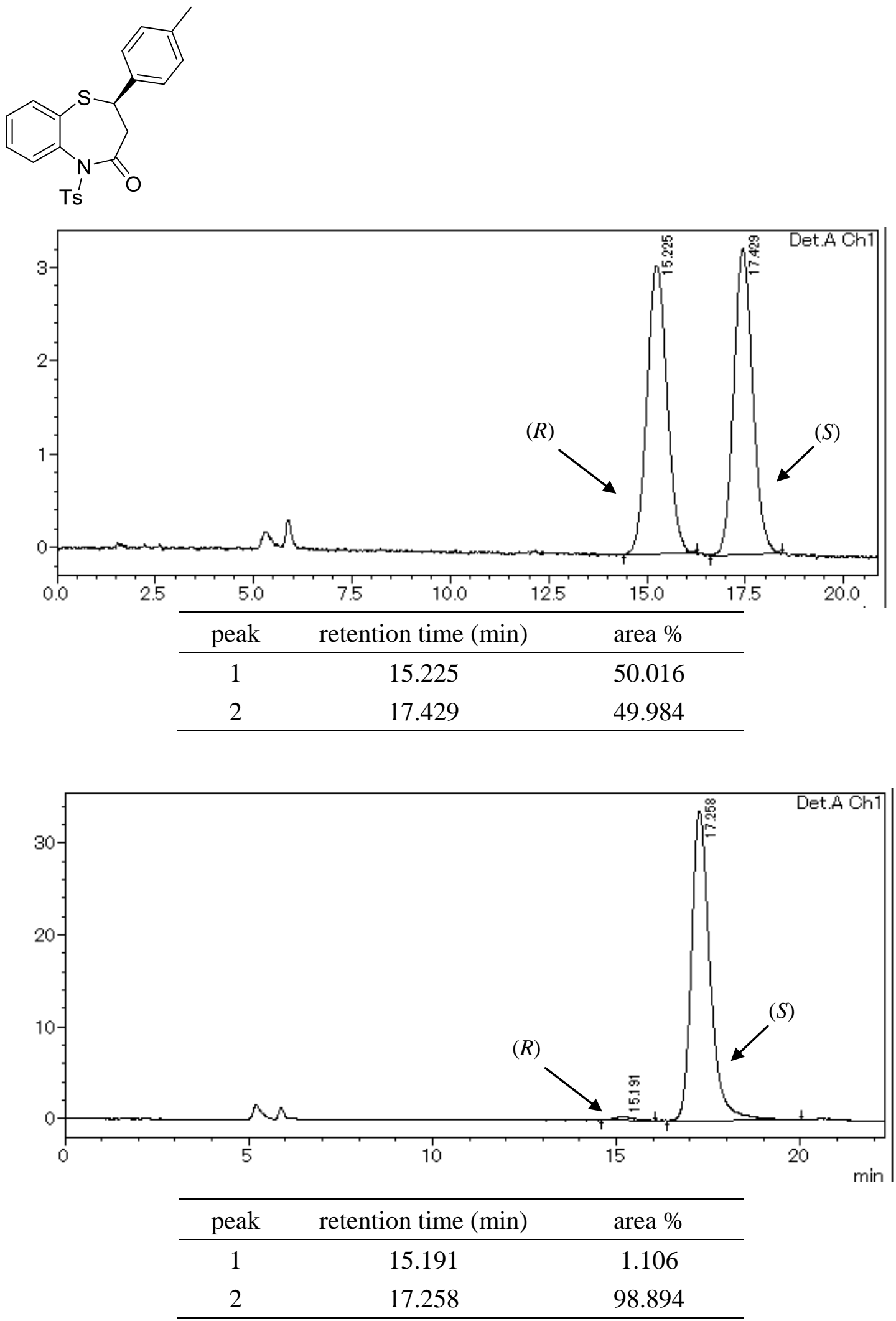
2-(2-Methylphenyl)-5-tosyl-2,3-dihydrobenzo[b][1,4]thiazepin-4(5H)-one (3g).
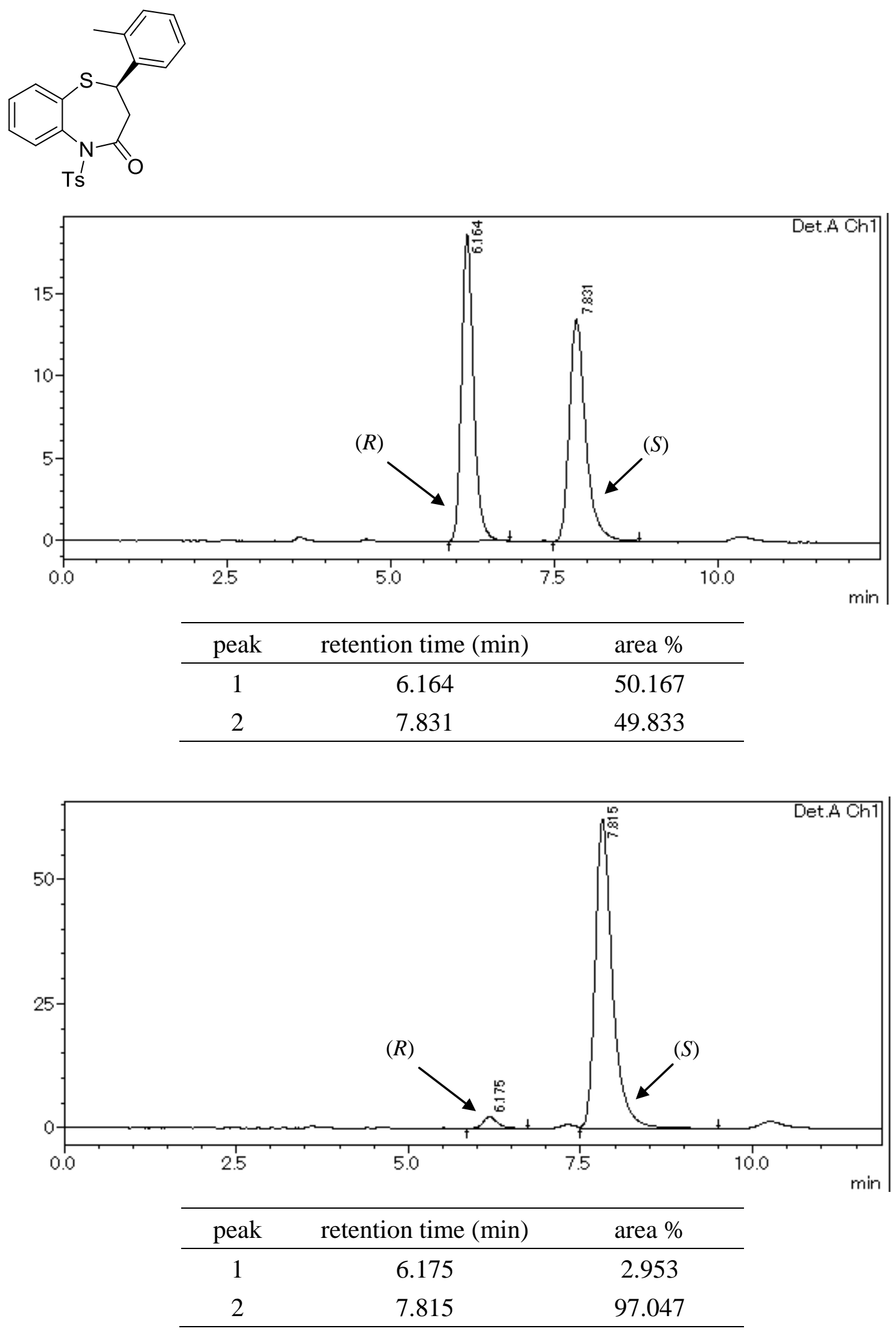
2-(4-Methoxyphenyl)-5-tosyl-2,3-dihydrobenzo[b][1,4]thiazepin-4(5H)-one (3h).<smiles>COc1ccc([C@@H]2CC(=O)N([As])c3ccccc3S2)cc1</smiles>

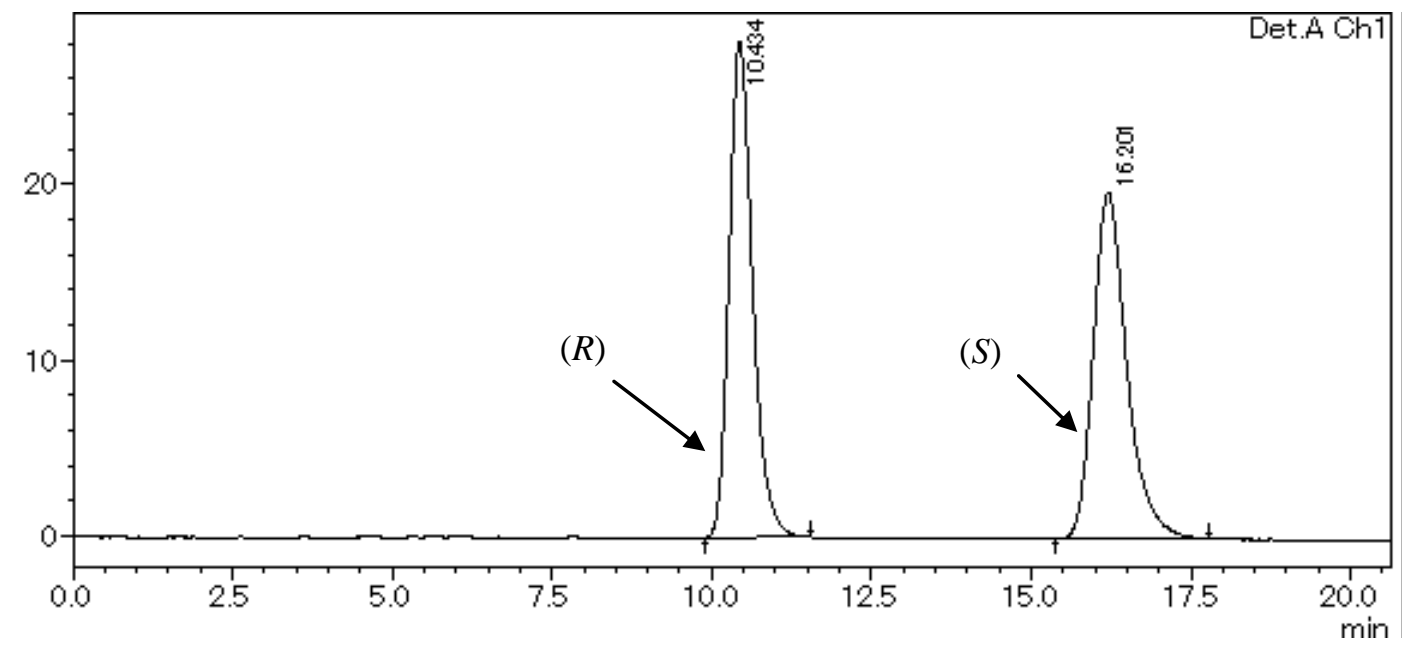

\begin{tabular}{ccc}
\hline peak & retention time $(\mathrm{min})$ & area $\%$ \\
\hline 1 & 10.434 & 50.001 \\
2 & 16.201 & 49.999
\end{tabular}

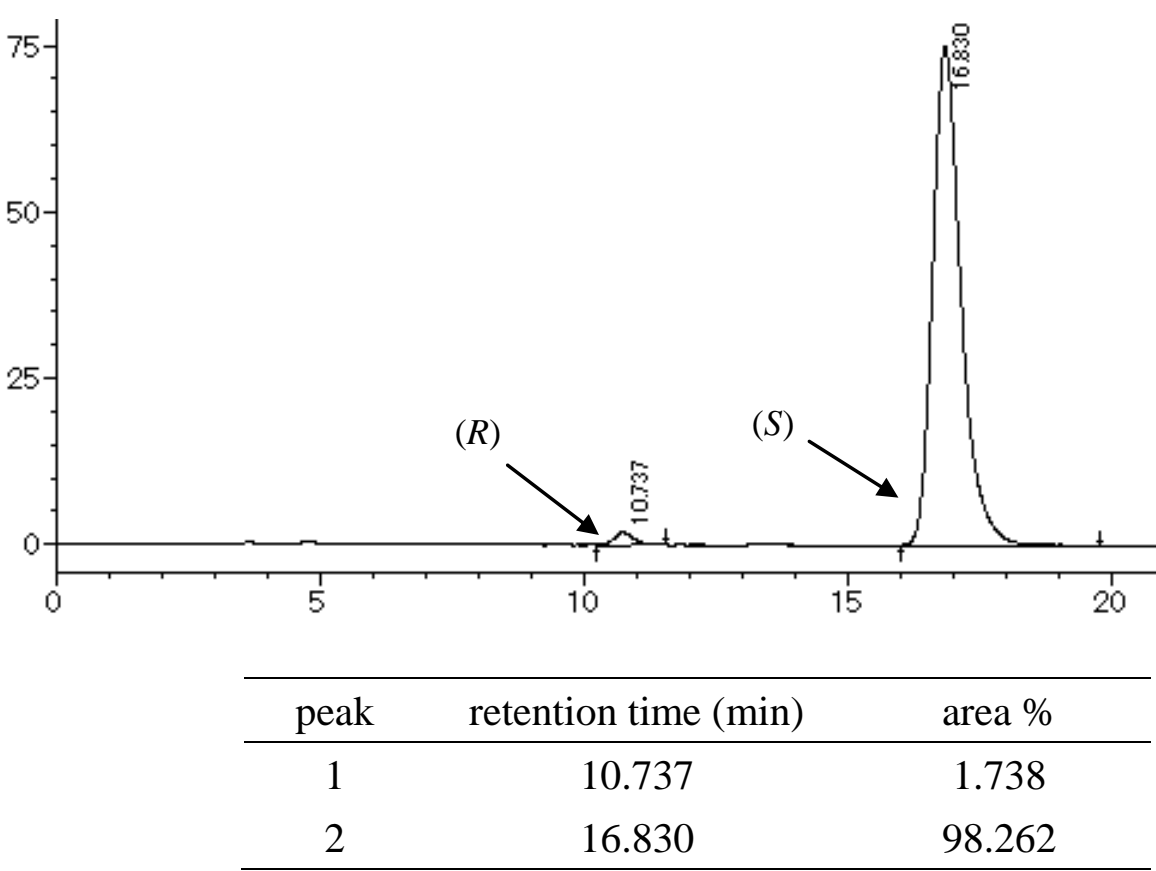

Det.A Ch1

$\min$ 
5-Tosyl-2-(4-(trifluoromethyl)phenyl)-2,3-dihydrobenzo[b][1,4]thiazepin-4(5H)-one (3i).<smiles>O=C1C[C@H](c2ccc(C(F)(F)F)cc2)Sc2ccccc2N1[TeH]</smiles>

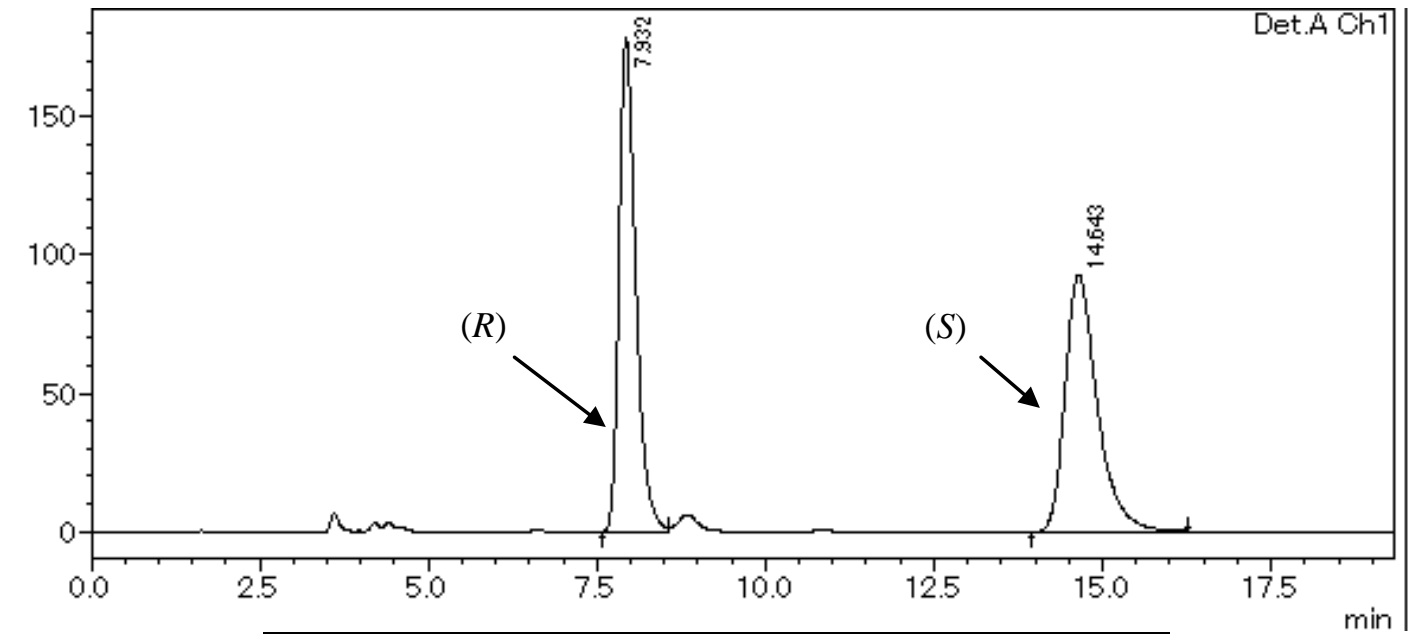

\begin{tabular}{ccc} 
peak & retention time $(\mathrm{min})$ & area $\%$ \\
\hline 1 & 7.932 & 50.082 \\
2 & 14.643 & 49.918
\end{tabular}

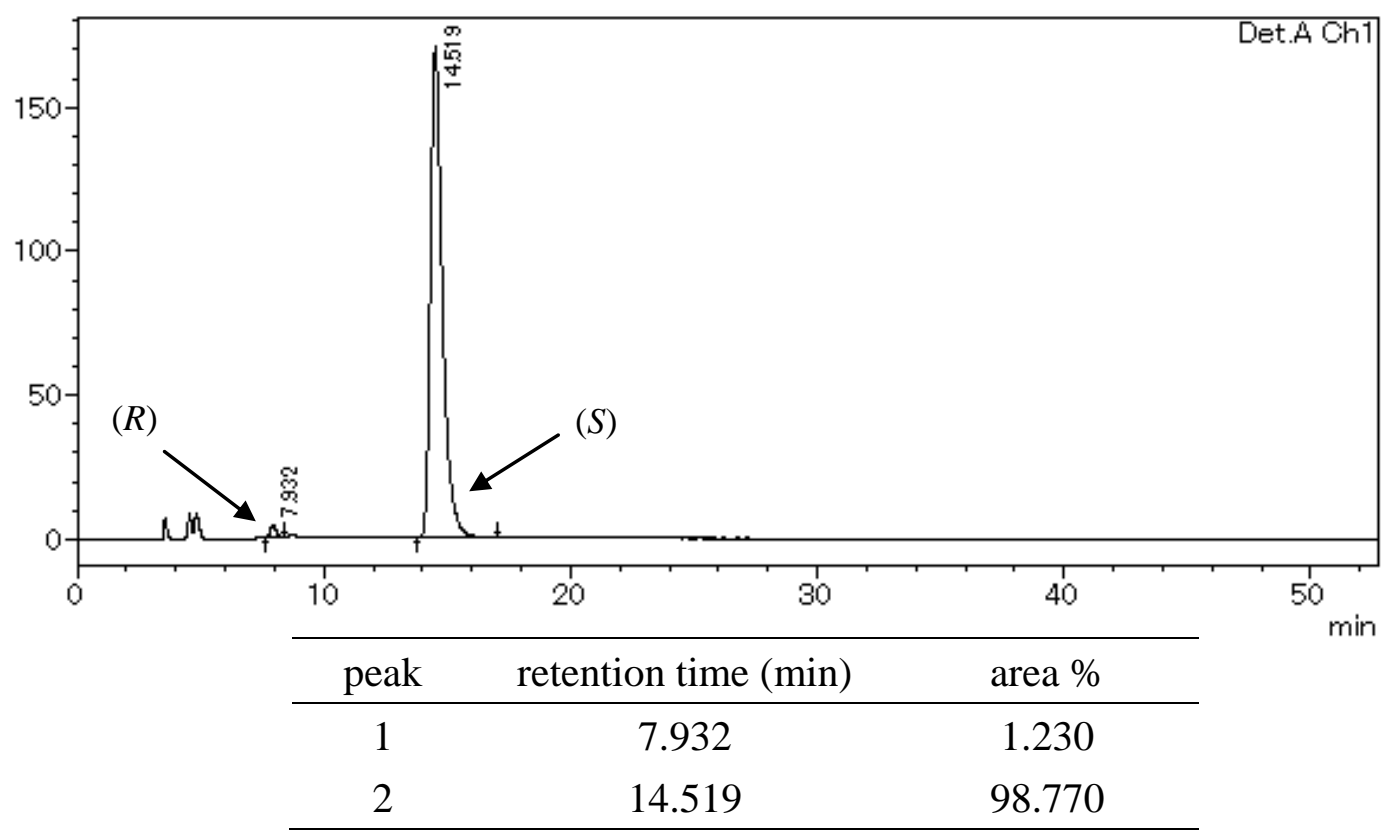


2-(4-Bromophenyl)-5-tosyl-2,3-dihydrobenzo[b][1,4]thiazepin-4(5H)-one $(3 \mathrm{j})$.
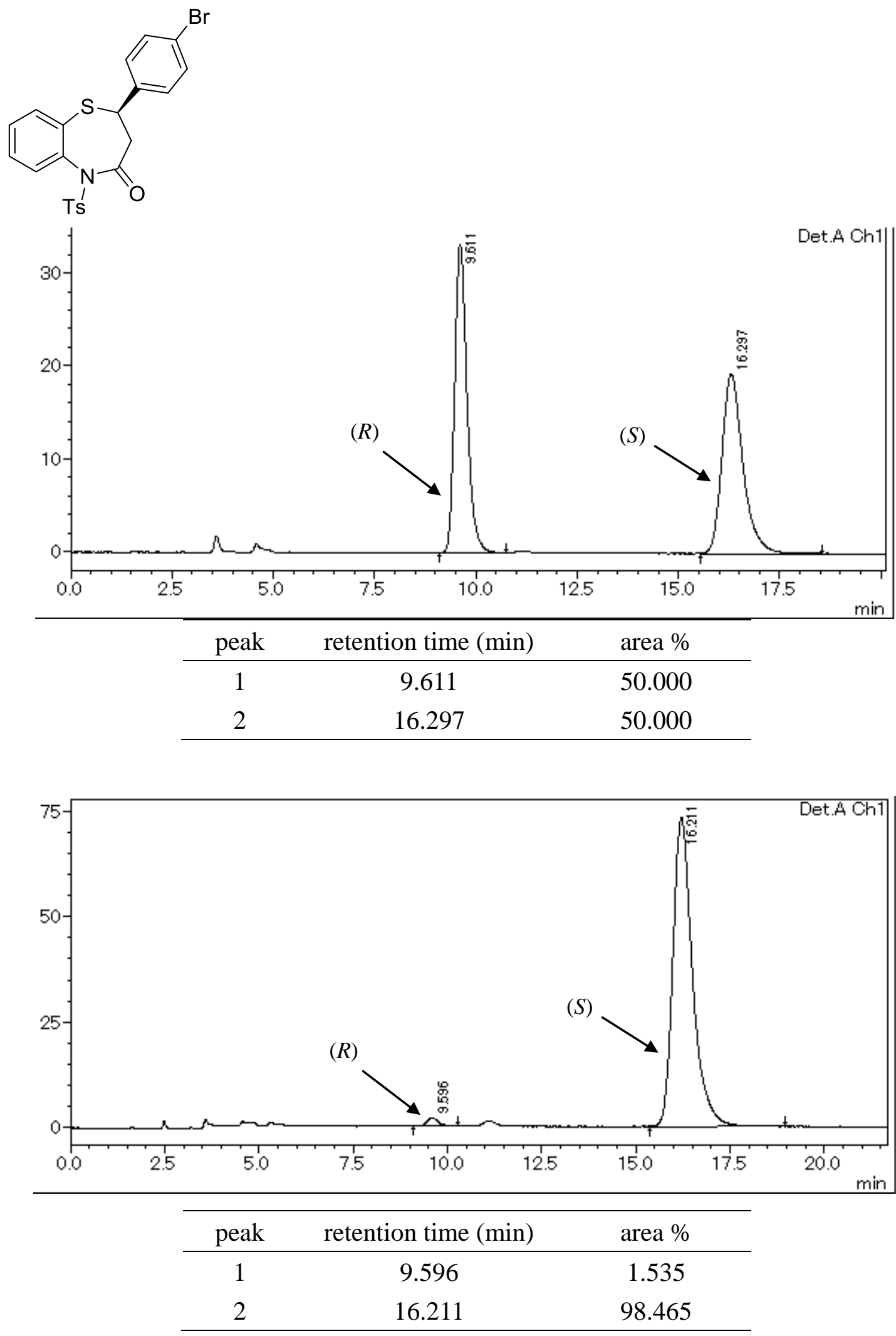
2-(Furan-2-yl)-5-tosyl-2,3-dihydrobenzo $[b][1,4]$ thiazepin-4(5H)-one (3k).<smiles>O=C1C[C@H](c2ccco2)Sc2ccccc2N1[TeH]</smiles>
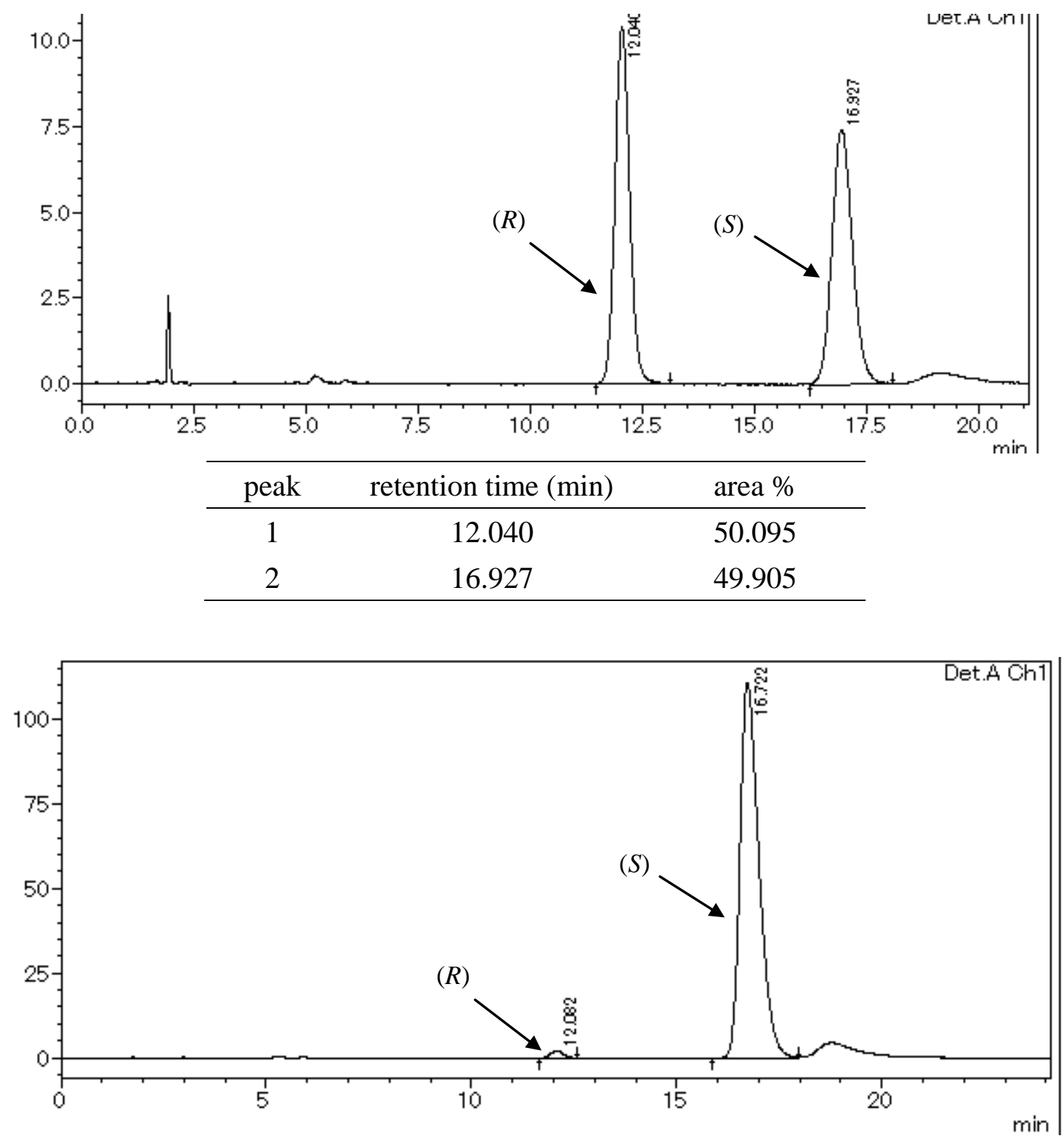

\begin{tabular}{ccc}
\hline peak & retention time $(\min )$ & area $\%$ \\
\hline 1 & 12.082 & 1.381 \\
2 & 16.722 & 98.619 \\
\hline
\end{tabular}


2-Ethyl-5-tosyl-2,3-dihydrobenzo $[b][1,4]$ thiazepin-4(5H)-one (3l).<smiles>[B]N1C(=O)CC(CC)Sc2ccccc21</smiles>

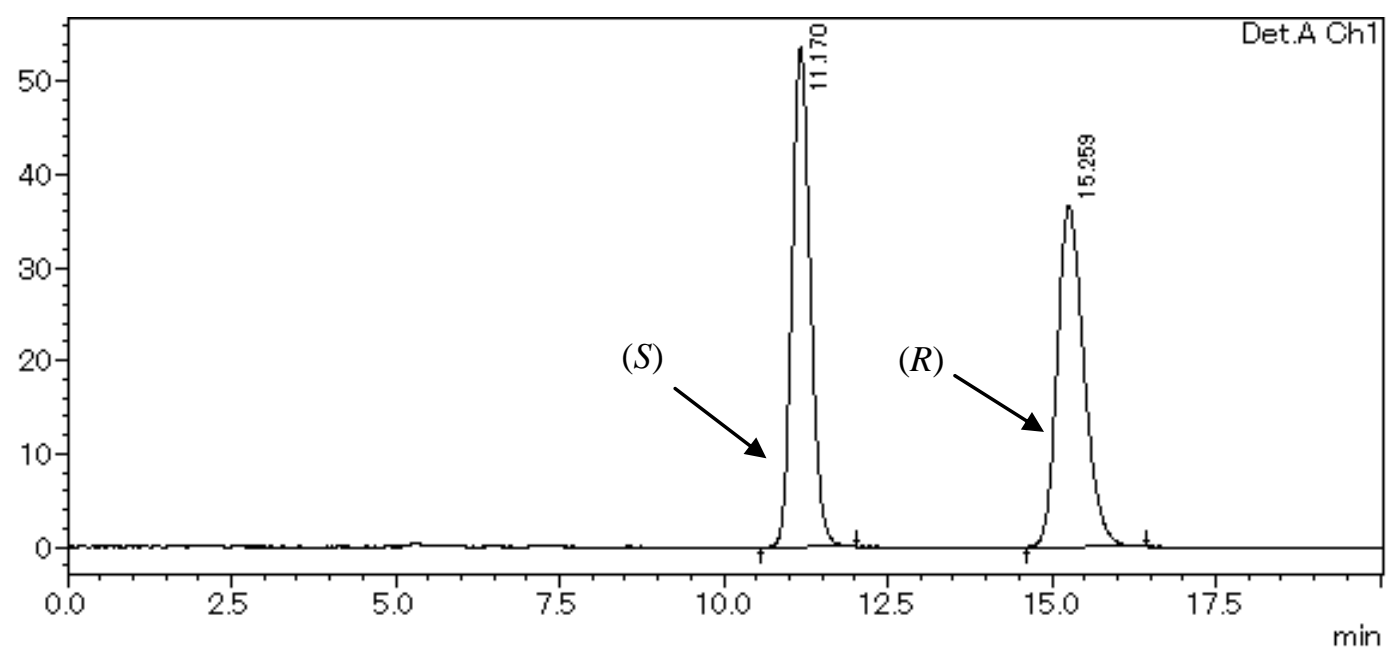

\begin{tabular}{ccc}
\hline peak & retention time $(\min )$ & area $\%$ \\
\hline 1 & 11.170 & 50.085 \\
2 & 15.259 & 49.915 \\
\hline
\end{tabular}

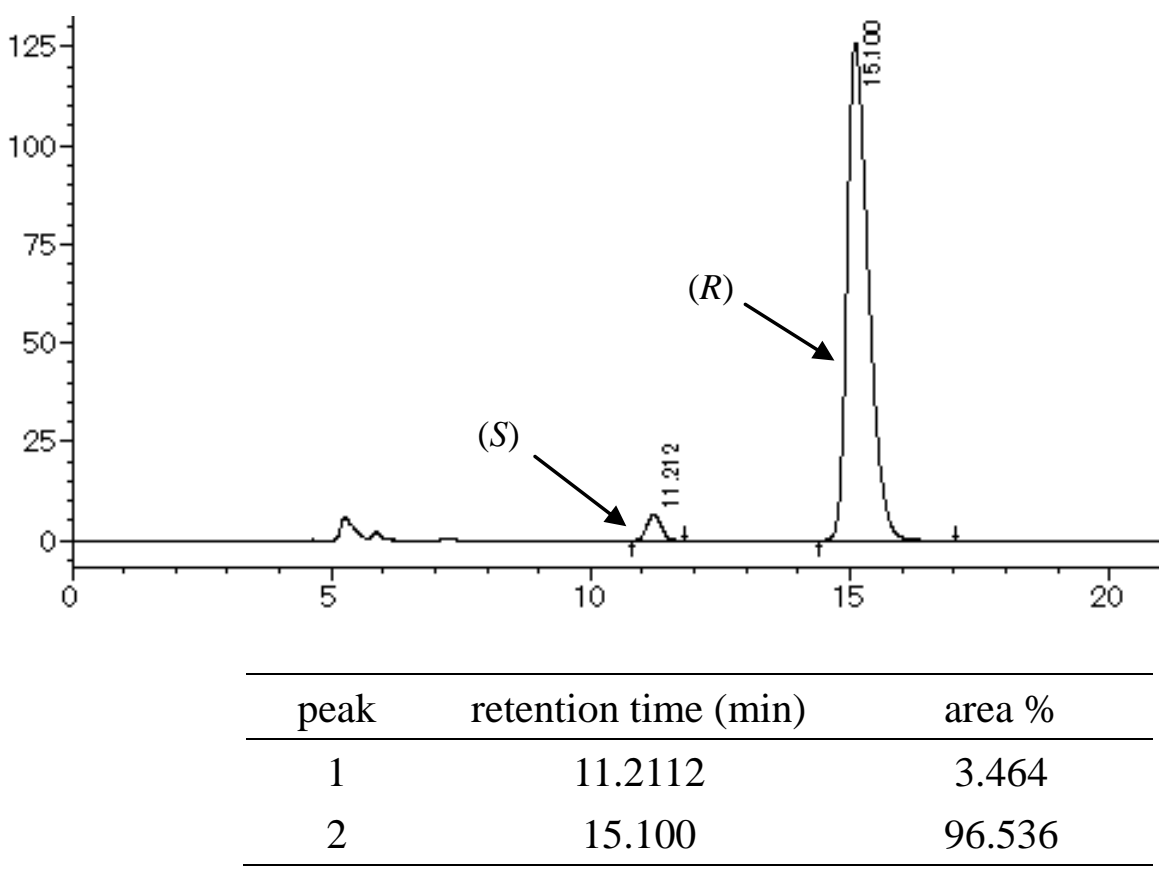

Det.A Ch1 
2-Isopropyl-5-tosyl-2,3-dihydrobenzo $[b][1,4]$ thiazepin-4(5H)-one (3m).<smiles>CC(C)C1CC(=O)N([As])c2ccccc2S1</smiles>

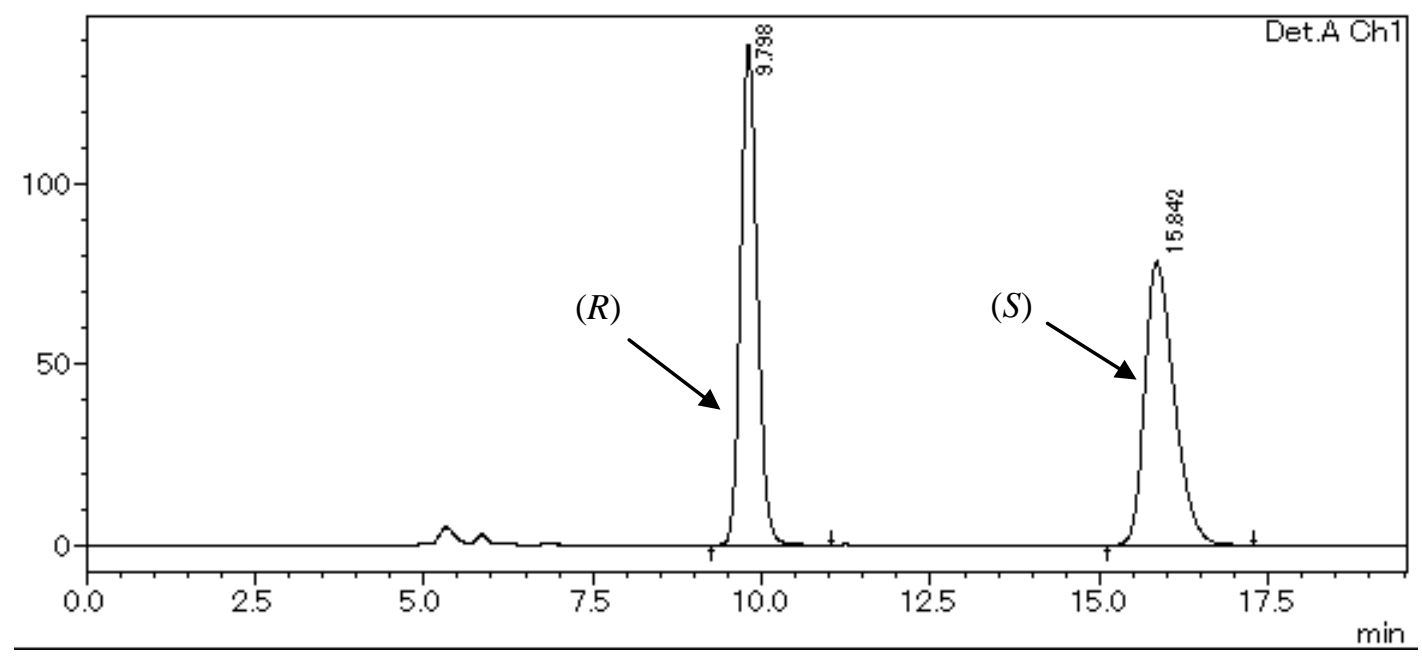

\begin{tabular}{ccc}
\hline peak & retention time $(\min )$ & area $\%$ \\
\hline 1 & 9.789 & 49.971 \\
2 & 15.842 & 50.029 \\
\hline
\end{tabular}

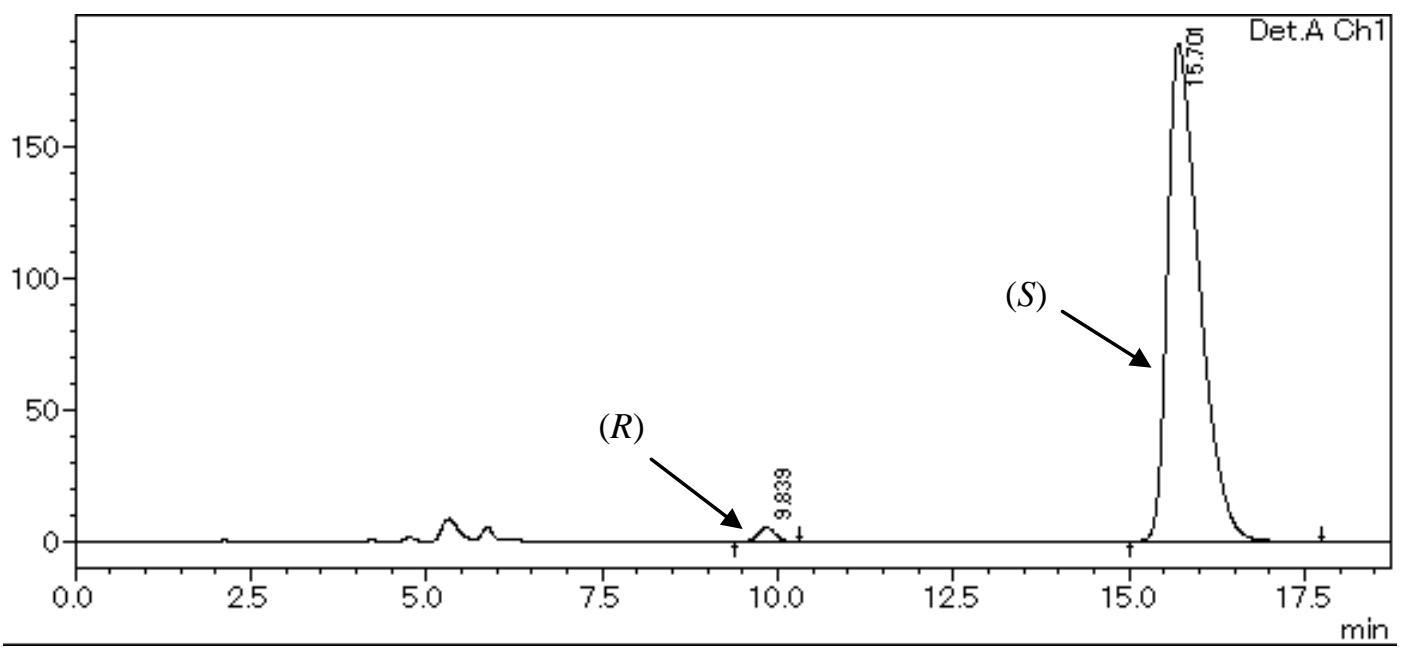

\begin{tabular}{ccc}
\hline peak & retention time $(\mathrm{min})$ & area $\%$ \\
\hline 1 & 9.839 & 1.426 \\
2 & 15.701 & 98.574 \\
\hline
\end{tabular}


7-Chloro-2-(p-tolyl)-5-tosyl-2,3-dihydrobenzo[b][1,4]thiazepin-4(5H)-one (3n).<smiles>Cc1ccc([C@@H]2CC(=O)N([As])c3cc(Cl)ccc3S2)cc1</smiles>
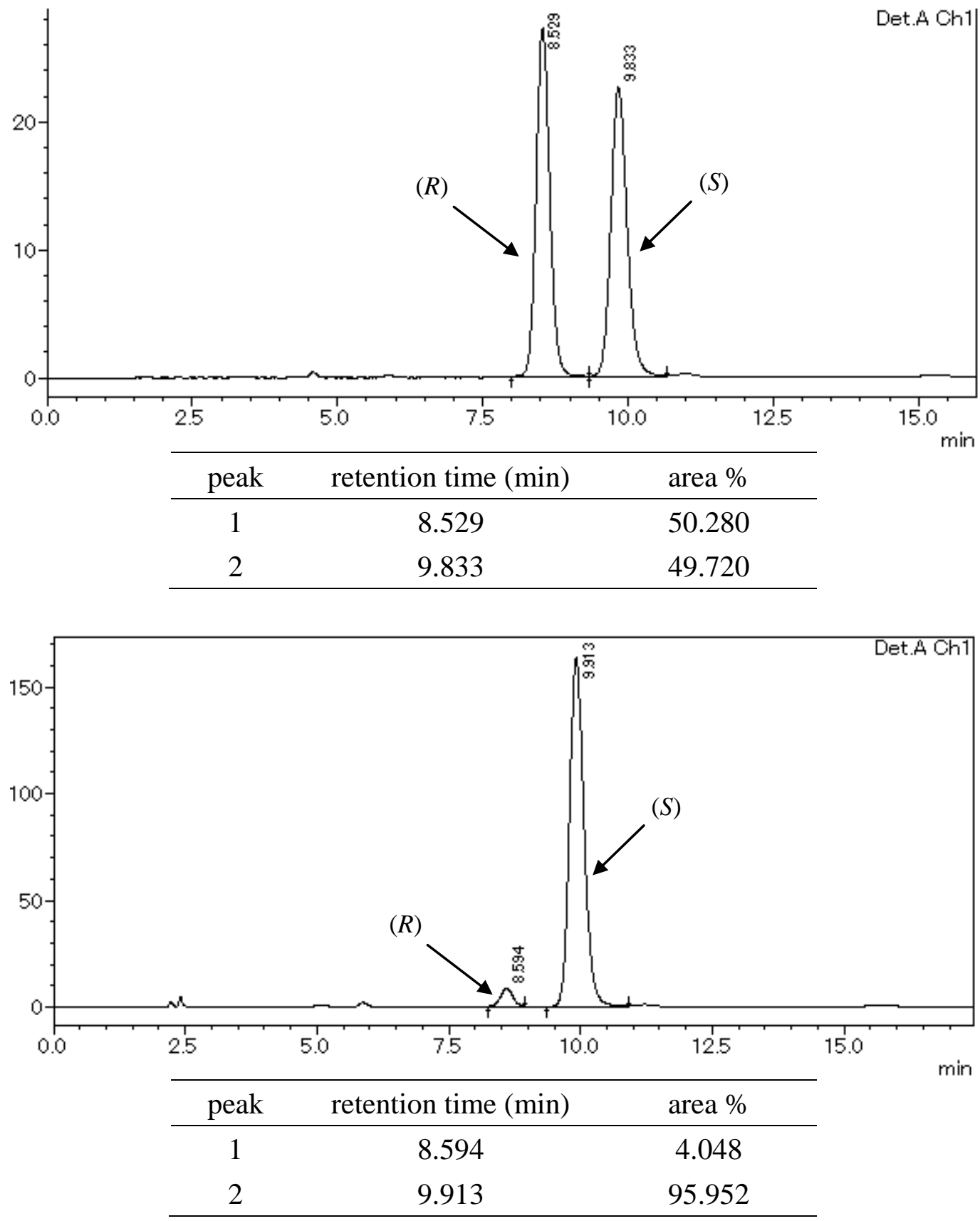
7-Chloro-2-(4-methoxyphenyl)-5-tosyl-2,3-dihydrobenzo $[b][1,4]$ thiazepin-4(5H)-on e (3o).<smiles>[B]N1C(=O)C[C@@H](c2ccc(OC)cc2)Sc2ccc(Cl)cc21</smiles>
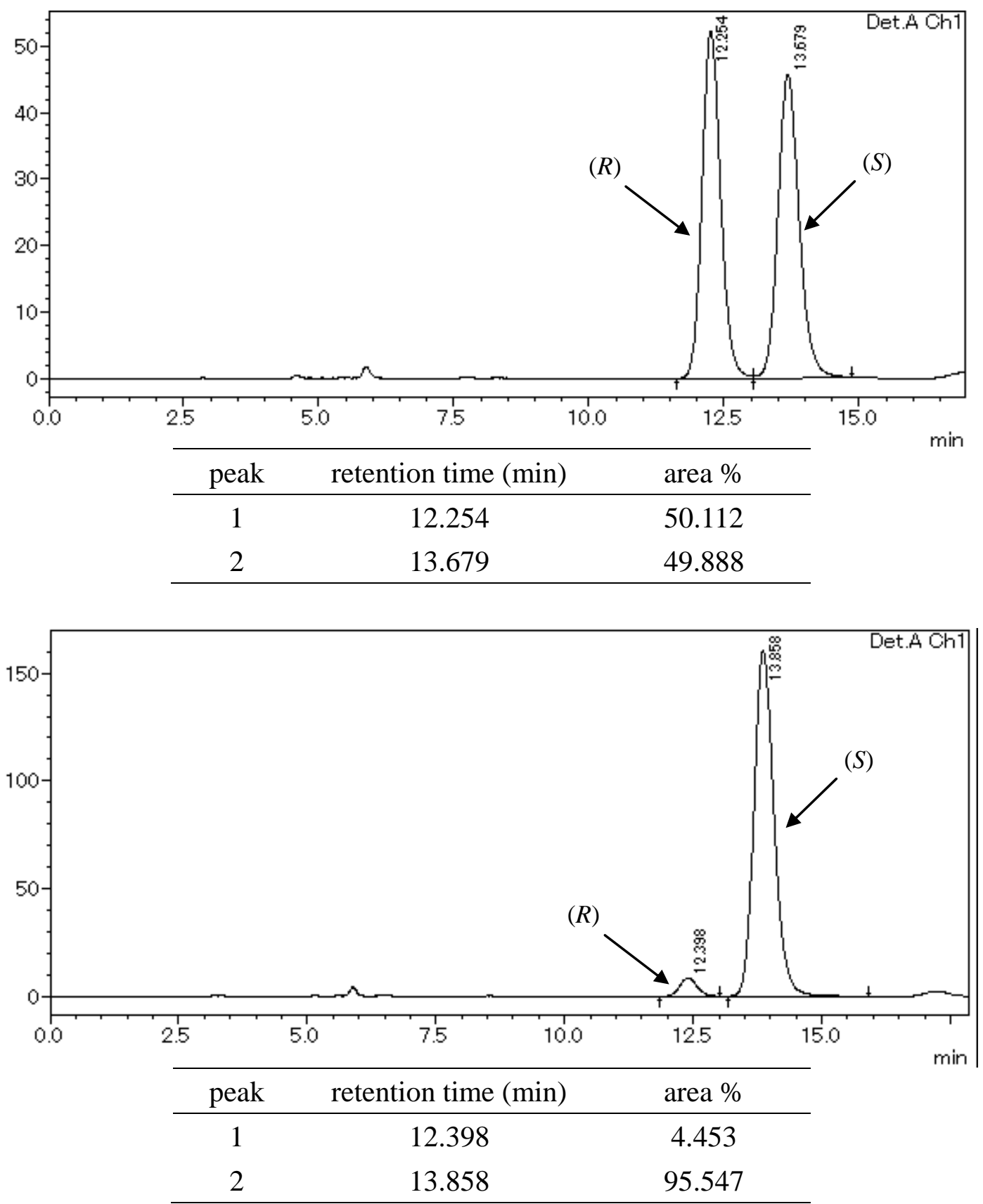
2-(4-Bromophenyl)-7-methyl-5-tosyl-2,3-dihydrobenzo $[b][1,4]$ thiazepin-4(5H)-one (3p).<smiles>[3H]N1C(=O)C[C@@H](c2ccc(Br)cc2)Sc2ccc(C)cc21</smiles>
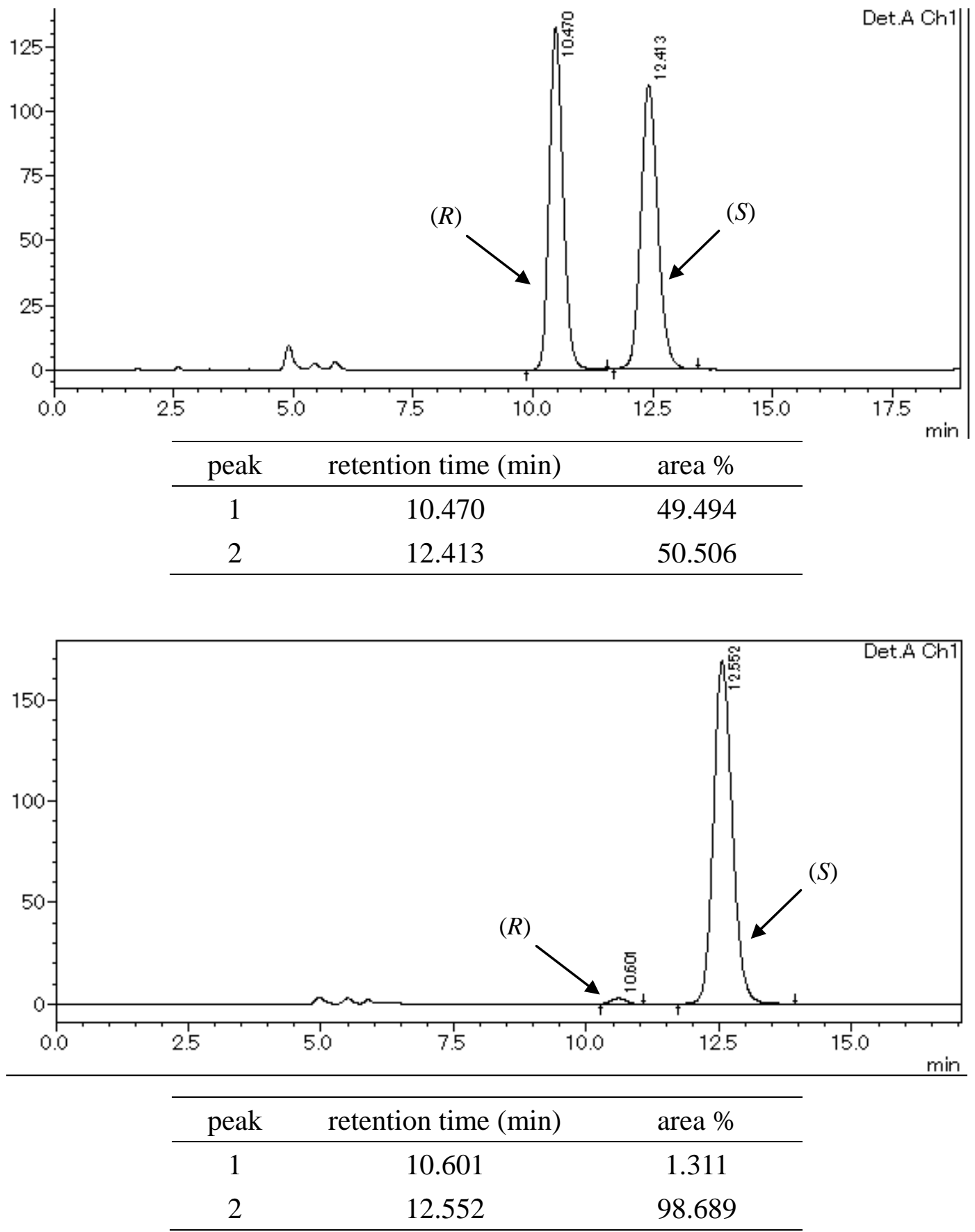
2-Ethyl-7-methoxy-5-tosyl-2,3-dihydrobenzo[b][1,4]thiazepin-4(5H)-one (3q).<smiles>CCC1CC(=O)N(S)c2cc(OC)ccc2S1</smiles>

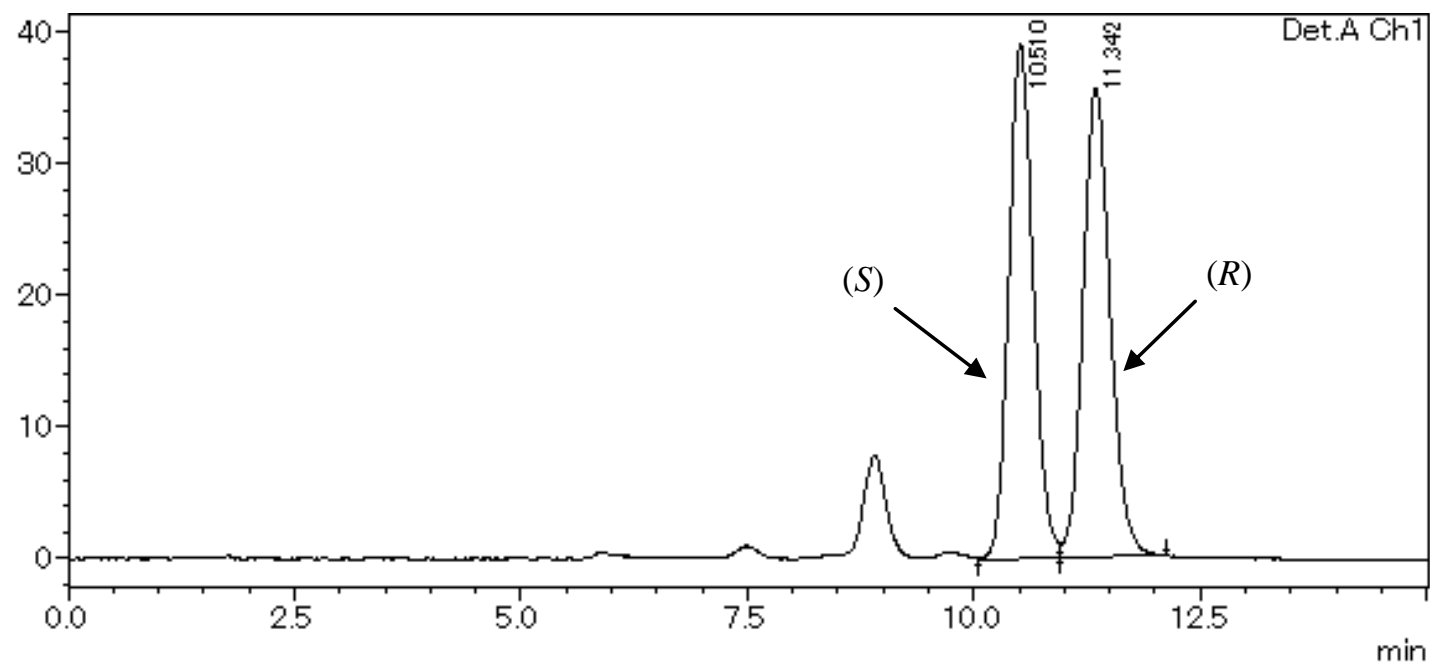

\begin{tabular}{ccc}
\hline peak & retention time $(\min )$ & area $\%$ \\
\hline 1 & 10.510 & 49.734 \\
2 & 11.342 & 50.266 \\
\hline
\end{tabular}

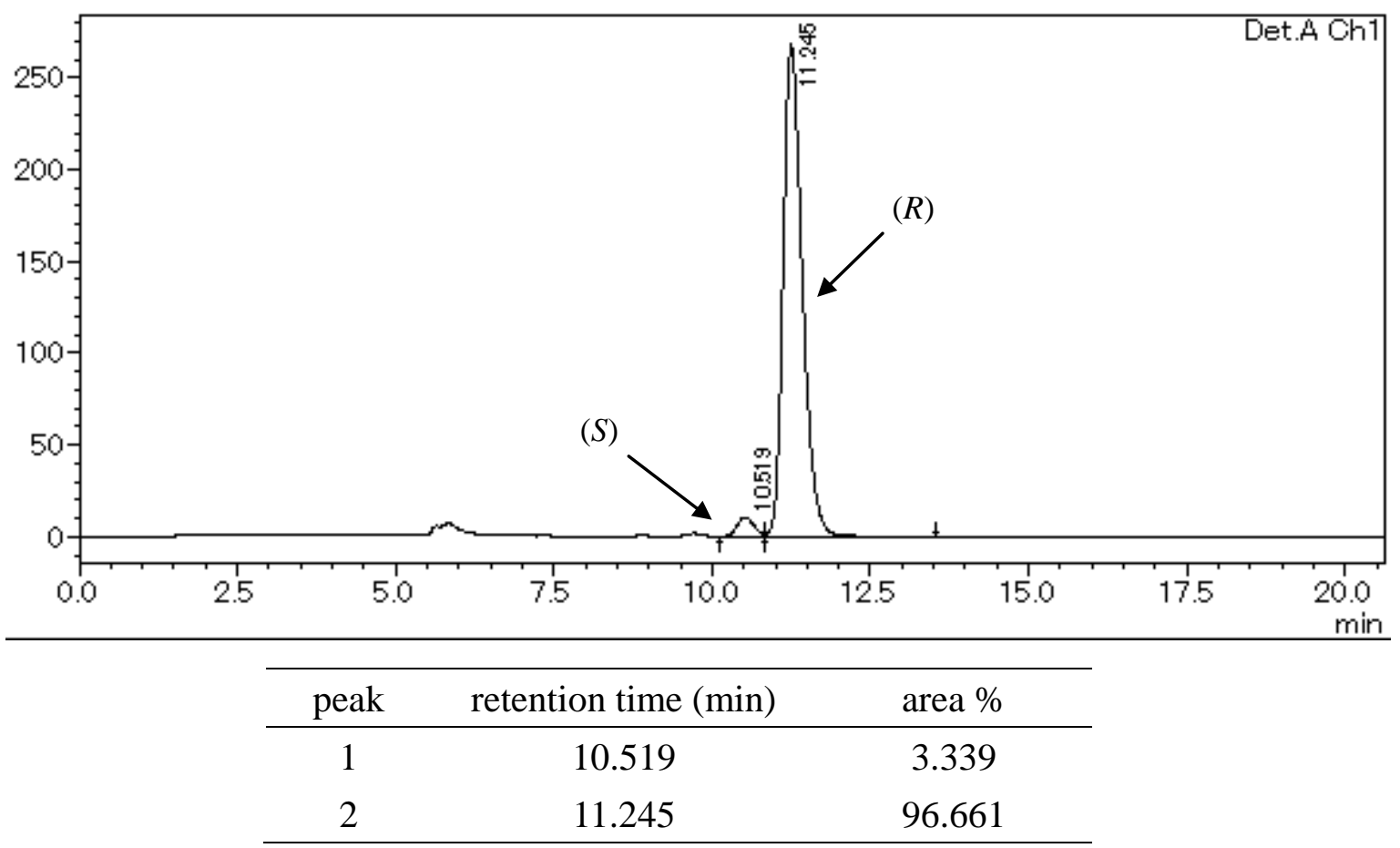


7-Bromo-2-(furan-2-yl)-5-tosyl-2,3-dihydrobenzo[b][1,4]thiazepin-4(5H)-one (3r).<smiles>O=C1C[C@H](c2ccco2)Sc2ccc(Br)cc2N1[Te]</smiles>

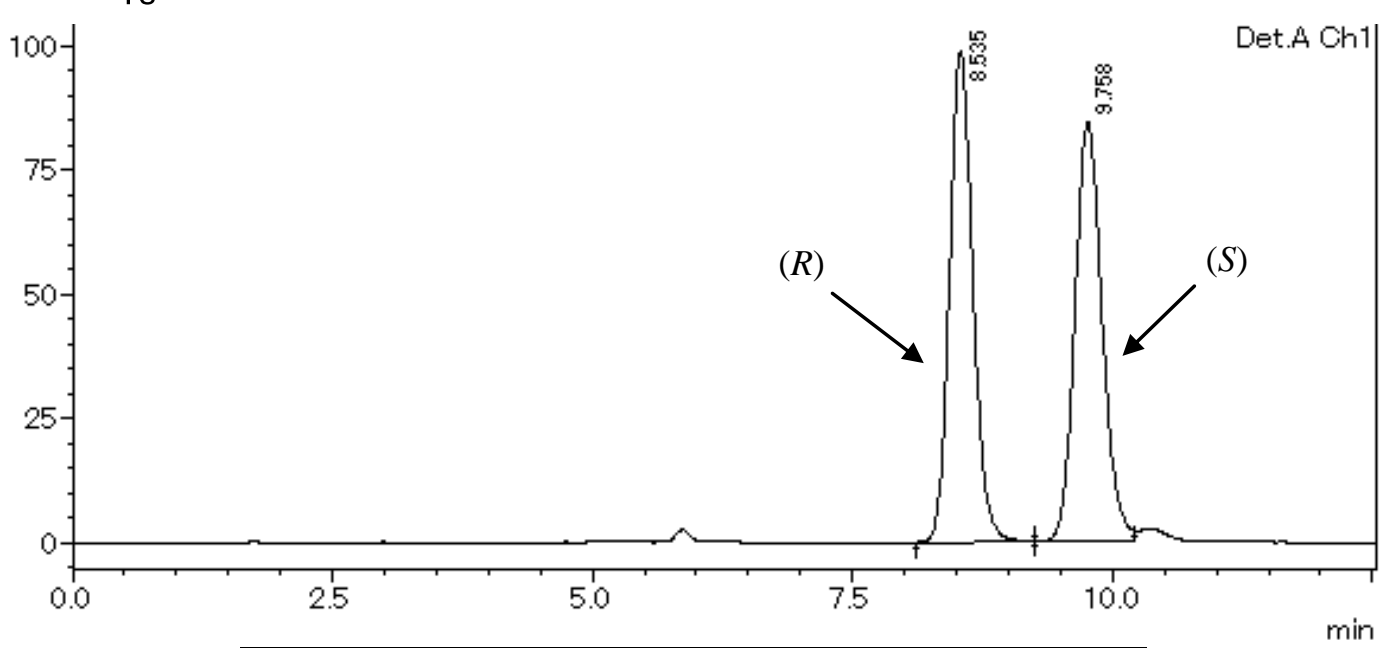

\begin{tabular}{ccc}
\hline peak & retention time $(\mathrm{min})$ & area $\%$ \\
\hline 1 & 8.535 & 50.268 \\
2 & 9.758 & 49.732 \\
\hline
\end{tabular}

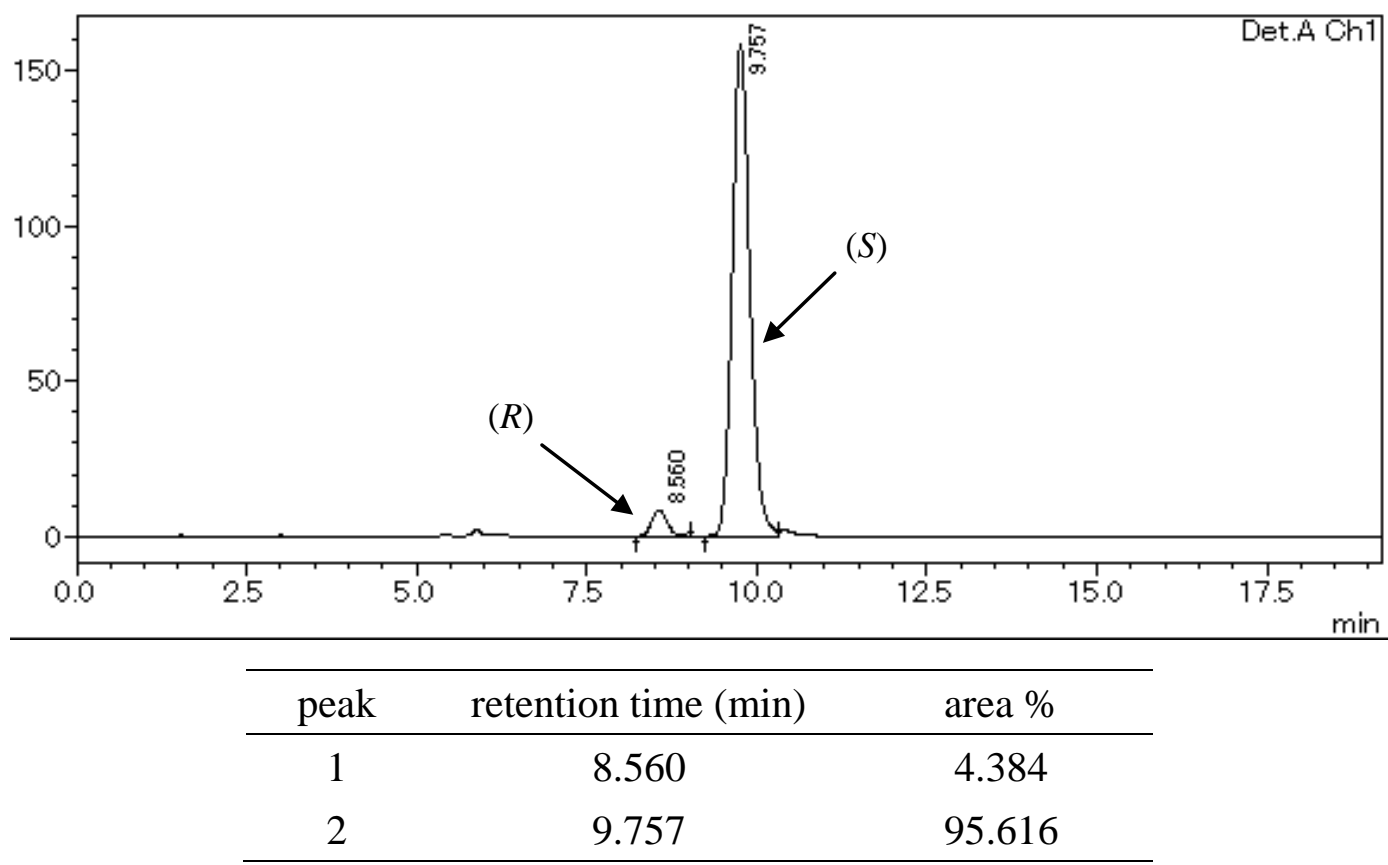


(R)-2-Phenyl-2,3-dihydrobenzo $[b][1,4]$ thiazepin-4(5H)-one (7).<smiles>O=C1CC(c2ccccc2)Sc2ccccc2N1</smiles>
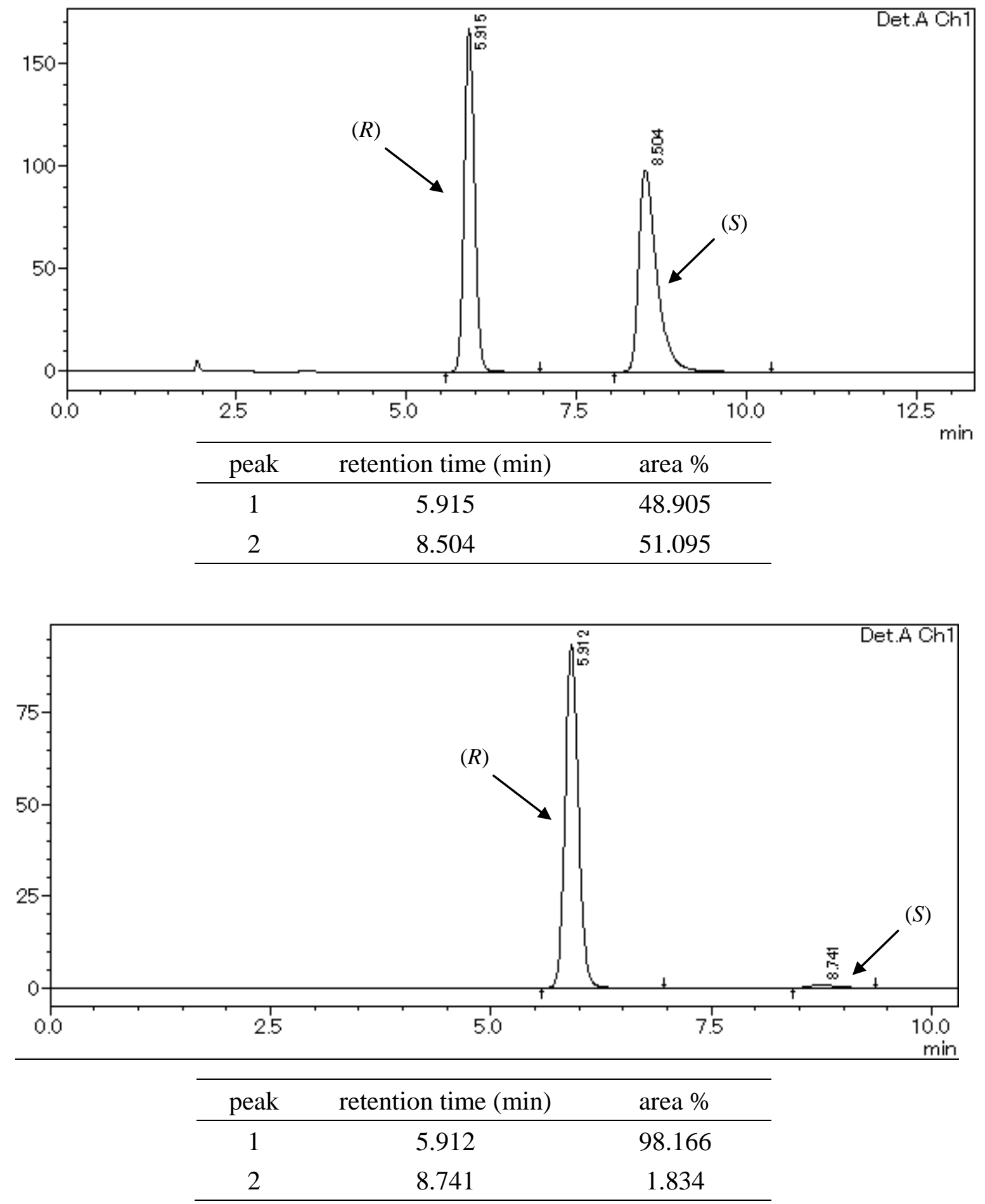
(R)-5-(2-(Dimethylamino)ethyl)-2-phenyl-2,3-dihydrobenzo[b][1,4]thiazepin-4(5H)one (8).
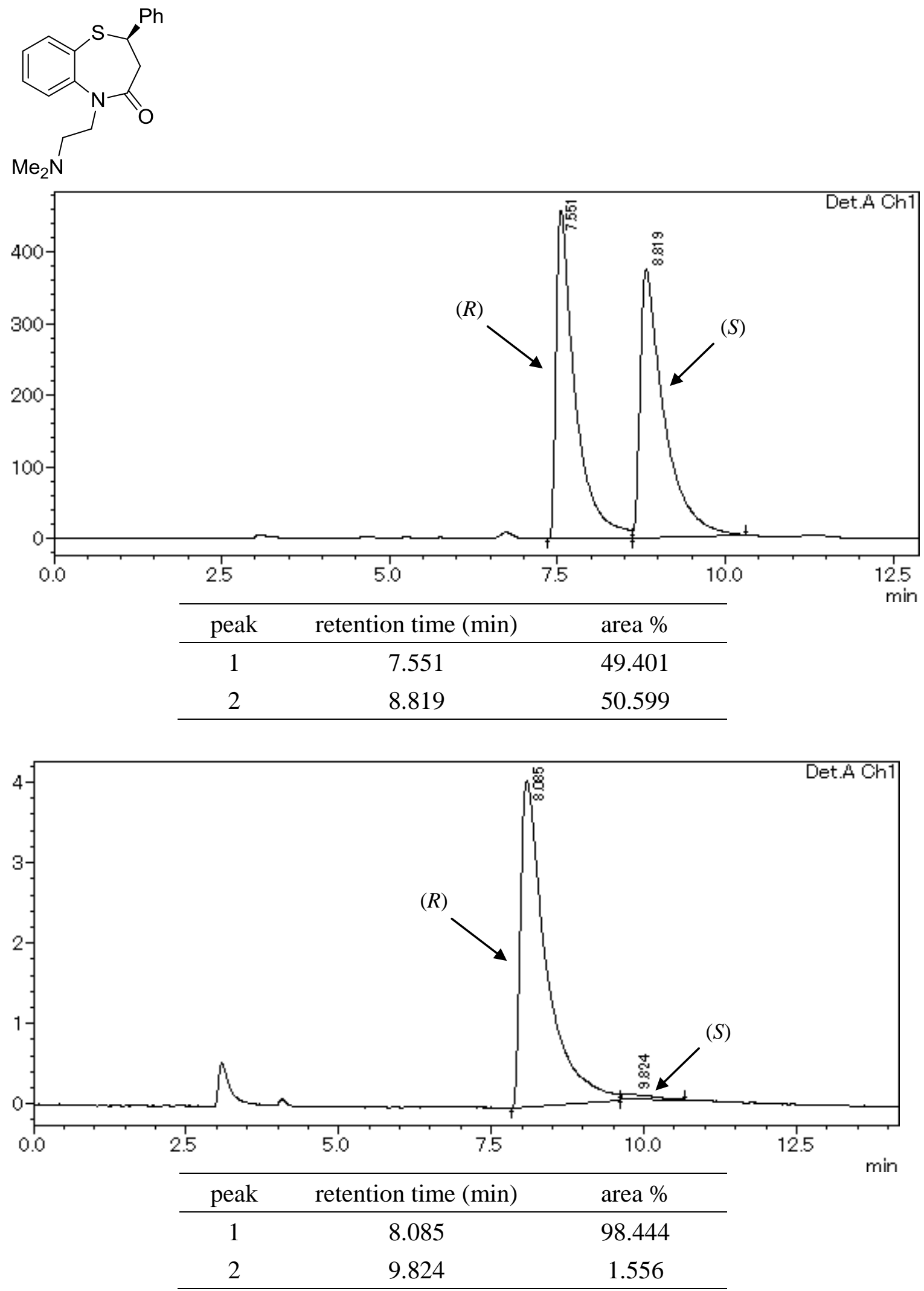


\section{ORTEP Drawing of 3a}

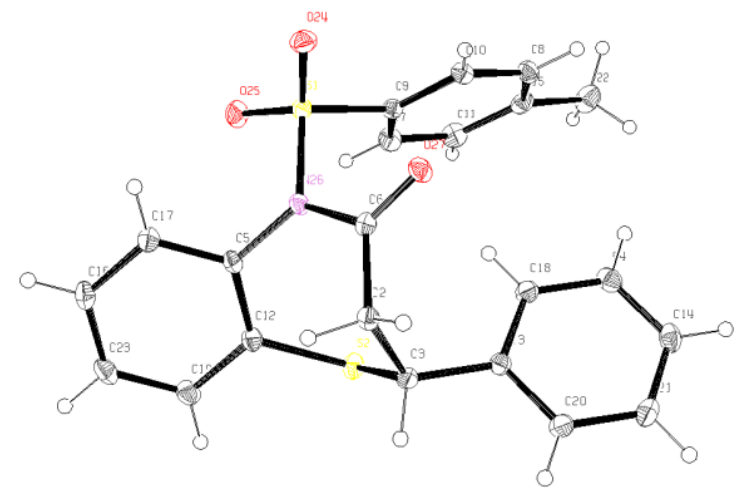

Identification code

Empirical Formula

Formula Weight

Crystal Color, Habit

Crystal Dimensions

Crystal System

Lattice Type

Lattice Parameters

Space Group

$\mathrm{Z}$ value

$\mathrm{D}_{\text {calc }}$

$\mathrm{F}_{000}$

$\mathrm{m}$ (MoKa)

Diffractometer

Radiation

Voltage, Current

Temperature

Detector Aperture

Data Images

$\omega$ oscillation Range

Exposure Rate

Detector Swing Angle

$\omega$ oscillation Range 3a

$\mathrm{C}_{22} \mathrm{H}_{19} \mathrm{NO}_{3} \mathrm{~S}_{2}$

409.52

colorless, prism

$0.460 \times 0.440 \times 0.200 \mathrm{~mm}$

orthorhombic

Primitive

$\mathrm{a}=12.4202(10) \AA \quad \alpha=90^{\circ}$

$\mathrm{b}=18.5765(15) \AA \quad \beta=90^{\circ}$

$\mathrm{c}=8.4080(7) \AA \quad \gamma=90^{\circ}$

$\mathrm{V}=1939.9(3) \AA^{3}$

$\mathrm{P} 2{ }_{1} 2{ }_{1} 2$ (\#18)

4

$1.402 \mathrm{~g} / \mathrm{cm}^{3}$

856.00

$2.980 \mathrm{~cm}^{-1}$

XtaLAB mini

Mo-K $\alpha(1=0.71075 \AA)$

graphite monochromated

$50 \mathrm{kV}, 12 \mathrm{~mA}$

$20.0^{\circ} \mathrm{C}$

$75 \mathrm{~mm}$ (diameter)

1080 exposures

$-60.0-120.0^{\circ}$

$64.0 \mathrm{sec} . /^{\circ}$

$30.00^{\circ}$

$-60.0-120.0^{\circ}$ 
Exposure Rate

Detector Swing Angle

$\omega$ oscillation Range

Exposure Rate

Detector Swing Angle

Detector Position

Pixel Size

2thetamax

No. of Reflections Measured

Corrections

Structure Solution

Refinement

Function Minimized

Least Squares Weights

2theta $\max$ cutoff

Anomalous Dispersion

No. Observations (All reflections)

No. Variables

Reflection/Parameter Ratio

Residuals: R1 (I>2.00sigma(I))

Residuals: R (All reflections)

Residuals: wR2 (All reflections)

Goodness of Fit Indicator

Flack Parameter

Max Shift/Error in Final Cycle

Maximum peak in Final Diff. Map

Minimum peak in Final Diff. Map
$64.0 \mathrm{sec} . /^{\circ}$

$30.00^{\circ}$

$-60.0-120.0^{\circ}$

$64.0 \mathrm{sec} . /^{\circ}$

$30.00^{\circ}$

$50.00 \mathrm{~mm}$

$0.146 \mathrm{~mm}$

$55.0^{\circ}$

Total: 19465

Unique: $4431\left(\mathrm{R}_{\mathrm{int}}=0.0154\right)$

Friedel pairs: 1905

Lorentz-polarization

Direct Methods

Full-matrix least-squares on $\mathrm{F}^{2}$

Sigma w $\left(\mathrm{Fo}^{2}-\mathrm{Fc}^{2}\right)^{2}$

$\mathrm{w}=1 /\left[\operatorname{sigma}^{2}\left(\mathrm{Fo}^{2}\right)+(0.0378 \cdot \mathrm{P})^{2}\right.$

$+0.3067 \cdot \mathrm{P}]$

where $\mathrm{P}=\left(\mathrm{Max}\left(\mathrm{Fo}^{2}, 0\right)+2 \mathrm{Fc}^{2}\right) / 3$

$55.0^{\circ}$

All non-hydrogen atoms

4431

253

17.51

0.0218

0.0221

0.0609

1.077

$0.01(4)$

0.000

$0.19 \mathrm{e}^{-} / \AA^{3}$

$-0.21 \mathrm{e}^{-} / \AA^{3}$ 